\title{
Compact Intracloud Discharges
}

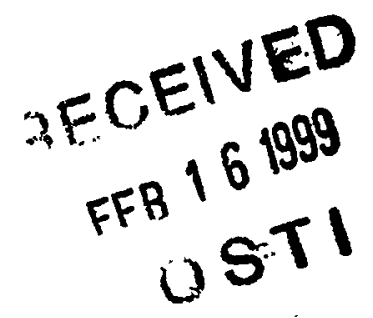

\section{Los Alamos \\ NATIONAL LABORATORY}

Los Alamos National Laboratory is operated by the University of Califormia for the United States Department of Energy under contract W-7405-ENG-36. 
This work was supported by the US Department of Energy, NN-20.

This thesis was accepted by the Department of Electrical Engineering, University of Colorado, Boulder, Colorado, in partial fulfillment of the requirements for the degree of Doctor of Philosophy. The text and illustrations are the independent work of the author and only the front matter has been edited by the CIC-1 Writing and Editing Staff to conform with Department of Energy and Los Alamos National Laboratory publication policies.

An Affirmative Action/Equal Opportunity Employer

This report was prepared as an account of work sponsored by an agency of the United States Government. Neither The Regents of the University of California, the United States Government nor any agency thereof, nor any of their employees, makes any warranty, express or implied, or assumes any legal liability or responsibility for the accuracy, completeness, or usefulness of any information, apparatus, product, or process disclosed, or represents that its use would not infringe privately owned rights. Reference herein to any specific commercial product, process, or service by trade name, trademark, manufacturer, or otherwise, does not necessarily constitute or imply its endorsement, recommendation, or favoring by The Regents of the University of California, the United States Government, or any agency thereof. The views and opinions of authors expressed herein do not necessarily state or reflect those of The Regents of the University of California, the United States Government, or any agency thereof. Los Alamos National Laboratory strongly supports academic freedom and a researcher's right to publish; as an institution, however, the Laboratory does not endorse the viewpoint of a publication or guarantee its technical correctness. 


\section{DISCLAIMER}

Portions of this document may be illegible in electronic image products. Images are produced from the best available original document. 


\section{Compact Intracloud Discharges}

\section{David A. Smith}




\section{ACKNOWLEDGMENTS}

I would like to take this opportunity to express my gratitude to the members of my dissertation committee, to my colleagues at Los Alamos National Laboratory (LANL) and the New Mexico Institute of Mining and Technology (NMIMT), to LANL and the Department of Energy for funding this research, and to my friends and family who have provided advice, support, and assistance to me since I began my graduate work five years ago.

Dan Holden, a Los Alamos technical staff member and a member of my committee, has been my mentor at Los Alamos for nine years. As well as being a good friend with whom I have shared countless adventures, Dan has been a tremendous positive influence on me and has provided me with numerous opportunities to learn and grow both personally and professionally. Thank you, Dan.

I would also like to express my gratitude to the other members of my University of Colorado (CU) graduate committee: Susan Avery, my advisor, Electrical Engineering faculty member, and Director of the Cooperative Institute for Research in Environmental Sciences; Jeffrey Forbes, Aerospace Engineering faculty member; Melinda Piket-May, Electrical Engineering faculty member, and Robert Serafin, Director of the National Center for Atmospheric Research. These people were very supportive of my cooperative research effort between the Department of Electrical 
Engineering at CU and the Space and Atmospheric Science group at Los Alamos, and provided valuable constructive feedback during our many sessions together.

Numerous workers at Los Alamos deserve recognition for their contributions to this research. The following present and former technical staff members of the Radio and Ionospheric Physics team took the time to share with me their expertise in disciplines ranging from thunderstorm electrification to ionospheric propagation: Paul Argo, Joe Fitzgerald, Abe Jacobson, Bob Massey, Charley Rhodes, Bob RousselDupré, and Xuan-Min Shao. In addition to thanking these people, I would also like to express my gratitude to Marx Brook and Paul Krehbiel of NMIMT, who both made significant contributions to my understanding of the physical processes that occur during thunderstorms. Countless Los Alamos personnel not already mentioned provided generously of their time and/or resources in assisting me with various aspects of the research detailed in this dissertation. These people include Dot DeLapp, Jim Devenport, Mary Dugan, Bob Franz, Dave Guenther, Phil Klingner, Eloisa Michel, Karen Olivas, Marty Shipley, Gary Stelzer, Brian Wiemers, and HongHong Zhu. I sincerely appreciate the assistance provided by these people. Bob Franz and Xuan-Min Shao deserve special mention for providing me with computer programs that they authored and that allowed me to complete portions of my analyses. I would also like to single out two people who were not on my committee, but who provided generously of their time to proofread entire drafts of my dissertation and provide me with valuable feedback: Bob Massey and my mom. 
Funding for this research was provided by the Department of Energy. I gratefully acknowledge their support and the enthusiastic support provided by Los Alamos National Laboratory and the Space and Atmospheric Science group, NIS-1.

Finally I would like to thank my friends and family for their loyal support, especially during these past four months as I chose to curtail many of my regular social and recreational activities in order to focus on the completion of my dissertation. My mom, dad, brother Leo, and best friend Shayna deserve special recognition here. Thank you to all of you. 


\section{CONTENTS}

CHAPTER

PAGE

1

INTRODUCTION

1

1.1

Blackbeard AND TransionosPheric PUlse PaIRs (TIPPS) .... 2

1.1 .1

One-Source Hypothesis

8

1.1.2

Two-Source Hypothesis

17

1.2

SUBIONOSPHERIC PULSE PAIRS (SIPPS)

19

1.2.1

Dispersed Subionospheric Pulse Pairs

20

1.2 .2

Undispersed Subionospheric Pulse Pairs

23

1.3

NARROW POSITTVE BIPOLAR PULSES (NPBPS)

25

1.4

COMPACT INTRACLOUD DISCHARGES (CIDS)

30

2

INSTRUMENTATION

32

2.1

ElECtric Field Change INSTRUMENTATION

33

2.1.1

Field Change Meters

35

2.1 .2

Field Change Meter Calibration

42

2.2

BROADBAND HF INSTRUMENTATION

45

2.2.1

Broadband HF Receivers

46 
2.2.2 Broadband HF System Calibration ................................... 50

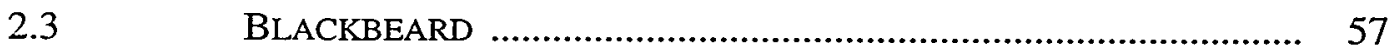

2.3.1 Blackbeard Timing Calibration …...................................... 61

3 SOURCE LOCATION METHODOLOGY ……....................................... 66

3.1 SOURCE LOCATIONS FROM GROUND-BASED MEASUREMENTS .... 72

3.1.1 Location Method One: Three or More TOA Receivers $\ldots . . . . .74$

3.1.2 Location Method Two: Two TOA Receivers …................... 80

3.1.3 Other Location Notes …………...................................... 84

3.2 SOURCE LOCATIONS FROM GROUND- AND SATELLITE-BASED

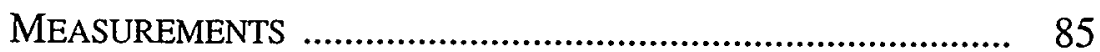

4 OBSERVATIONS FROM THREE SOUTHWESTERN THUNDERSTORMS ... 86

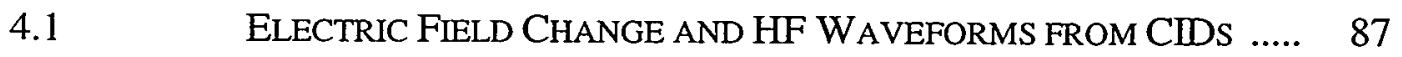

4.2 WAVEFORMS FROM CLOUD-TO-GROUND. AND INTRACLOUD

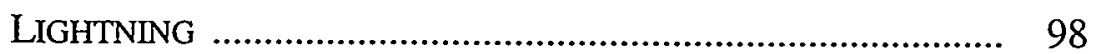

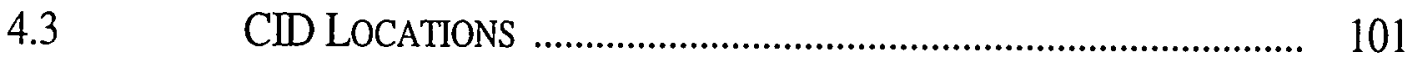

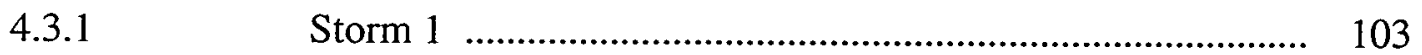

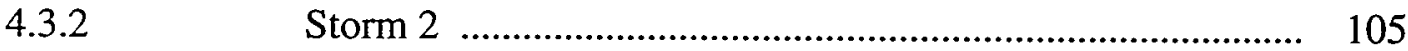

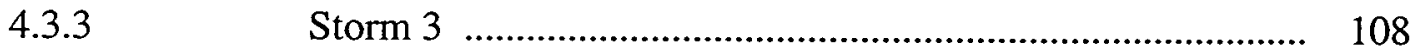

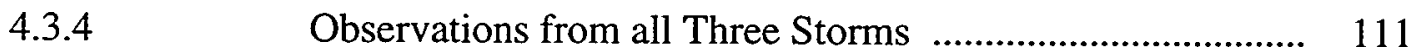

4.4 CID TEMPORAL CONTEXT ….................................................... 117 
Temporal Context of the Events Recorded on 11

Location Technique One: Source Height and Pulse

Separations 155

5.3 OVERVIEW OF COINCIDENT GROUND AND SATELLITE 


\section{TABLES}

NUMBER

PAGE

2. Instrumentation

$2.1 \quad$ Electric field change meter characteristics

41

4. Observations from Three Southwestern Thunderstorms

4.1 Source and ionosphere height calculations

5. Observations from Tropical Cyclone Fausto

5.1 HF propagation model inputs and outputs 154

6. CID Waveforms and Phenomenology

6.1 Mean NPBP characteristics

195

6.2 Comparison of NPBP initial and overshoot peak

Amplitudes 202

6.3

Results from VLF/LF propagation model

206

6.4 Comparison of amplitudes of NPBPs and IC/CG

waveforms

207 
7. CID Physical Characteristics 


\section{FIGURES}

1. Introduction

1.1

1.2

$$
\text { Spectrogram of TIPP event }
$$

One-source versus two-source hypotheses

Illustration of source/receiver geometry

Pulse time separation as a function of elevation angle

TIPP separation histogram

Dispersed SIPP

Dispersed lightning

Undispersed SIPP

NPBP from Le Vine [1980]

NPBP from Willett et al. [1989]
6

2. Instrumentation 
Block diagram of LANL electric field change meter

Computer wire-frame model of discone antenna

3. Source Location Methodology

4. Observations from Three Southwestern Thunderstorms 


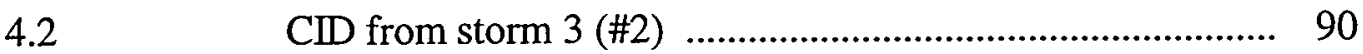

4.3 Multiple-hop NPBP from storm over Mexico ..................... 92

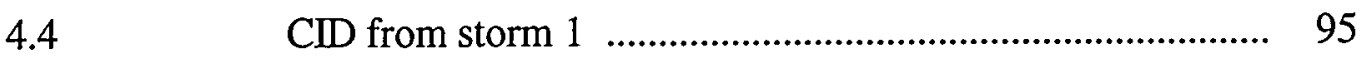

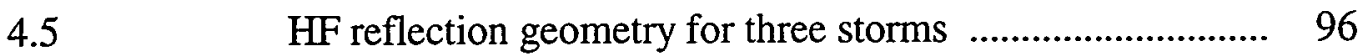

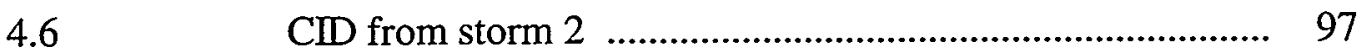

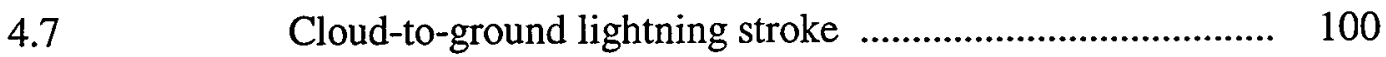

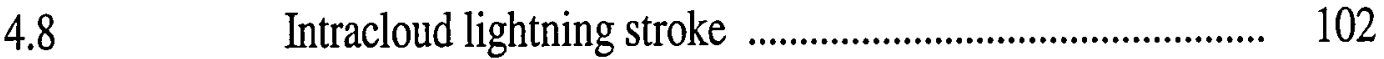

S.9 Storm 1 plan view source locations ……............................... 104

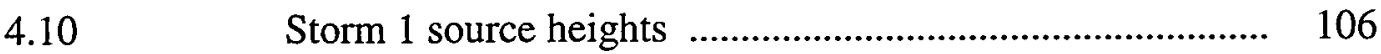

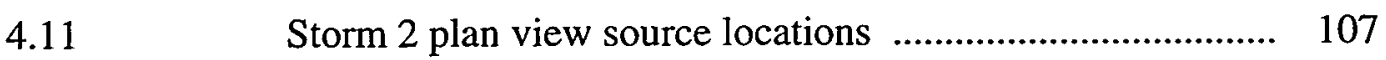

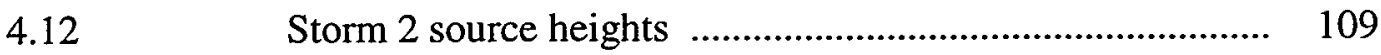

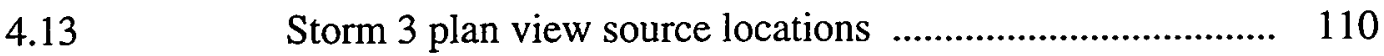

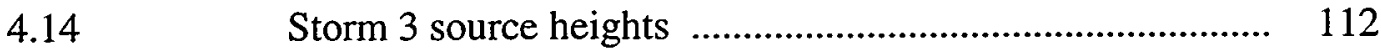

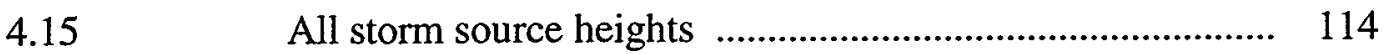

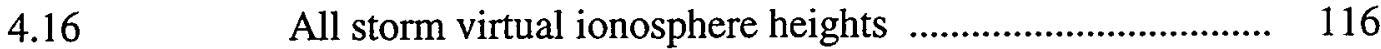

4.17 Storm time histories ….................................................. 119

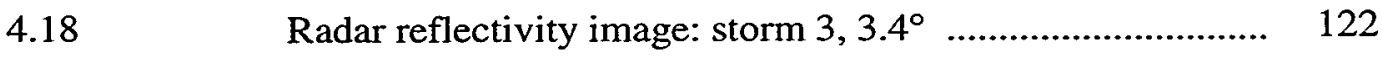

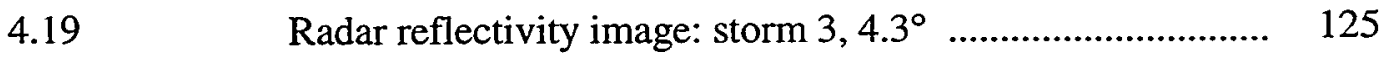

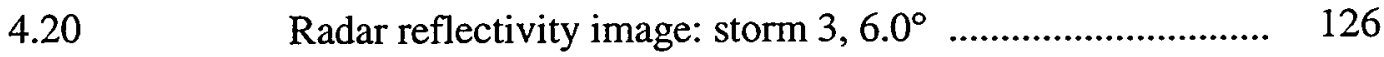

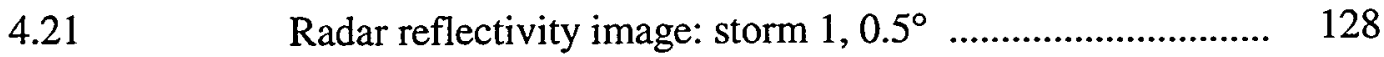

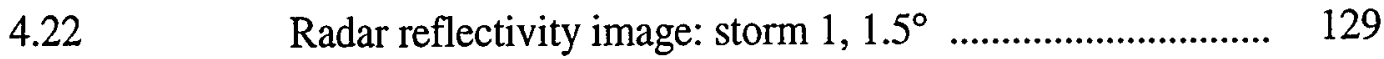

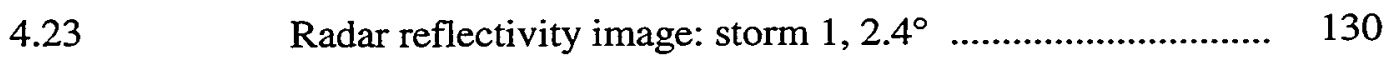


5. Observations from Tropical Cyclone Fausto

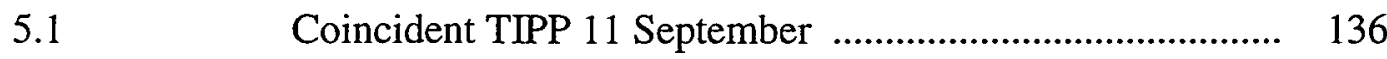

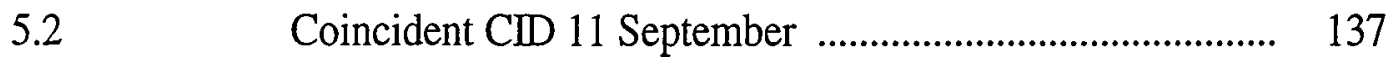

5.3 Whitened coincident CID 11 September $\ldots \ldots \ldots \ldots \ldots \ldots \ldots \ldots \ldots . . . \ldots \ldots$

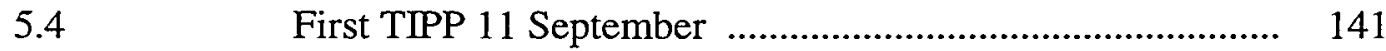

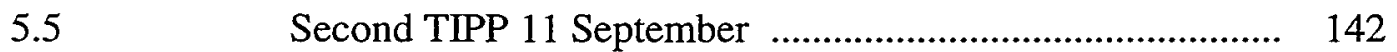

5.6 Sub-satellite points and fields of view 11 September ........ 144

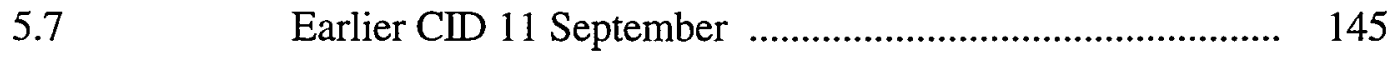

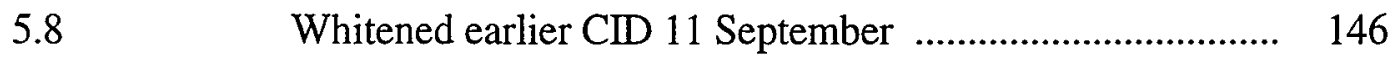

$5.9 \quad$ Time line for 11 September event detections $\quad \ldots \ldots \ldots \ldots \ldots \ldots \ldots \ldots . . . . . . .148$

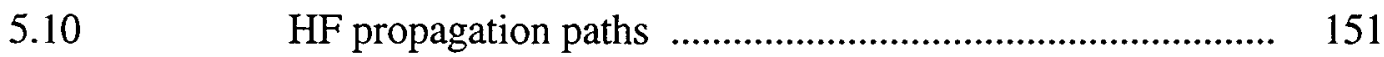

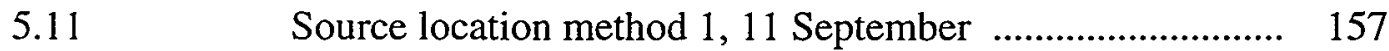

S.12 Source location method 1, zoom, 11 September ............... 159

$5.13 \quad$ Source location method 2, 11 September $\ldots \ldots \ldots \ldots \ldots \ldots \ldots \ldots . . . . \ldots 1$

5.14 Source location methods 1 and 2, 11 September ............... 163

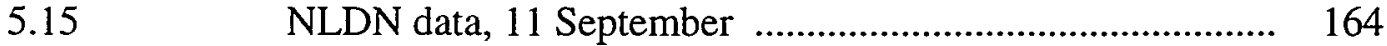

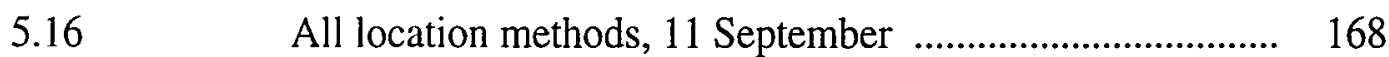

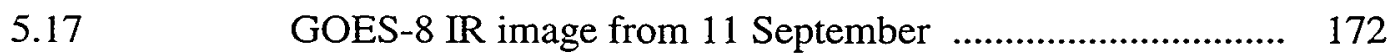

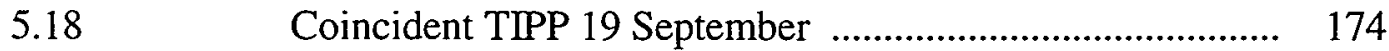

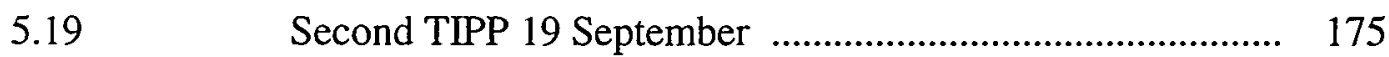

5.20 Sub-satellite points and fields of view 19 September ........ 176

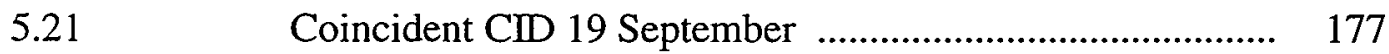


6. CID Waveforms and Phenomenology

\section{CID Physical Characteristics} Physical characteristics as a function of streamer 
7.4 Scatter plot of average current versus spatial extent $\ldots \ldots \ldots \ldots . . . \quad 244$ 


\title{
COMPACT INTRACLOUD DISCHARGES
}

\author{
David A. Smith
}

\begin{abstract}
In November of 1993, mysterious signals recorded by a satellite-borne broadband VHF radio science experiment called Blackbeard led to a completely unexpected discovery. Prior to launch of the ALEXIS satellite, it was thought that its secondary payload, Blackbeard, would most often detect the radio emissions from lightning when its receiver was not overwhelmed by noise from narrowband communication carriers. Instead, the vast majority of events that triggered the instrument were isolated pairs of pulses that were one hundred times more energetic than normal thunderstorm electrical emissions. The events, which came to be known as TIPPs (for transionospheric pulse pairs), presented a true mystery to the geophysics community. At the time, it was not even known whether the events had natural or anthropogenic origins. After two and one half years of research into the unique signals, two ground-based receiver arrays in New Mexico first began to detect and record thunderstorm radio emissions that were consistent with the Blackbeard observations. On two occasions, the ground-based systems and Blackbeard even recorded emissions that were produced by the same exact events. From the ground-
\end{abstract}


based observations, it has been determined that TIPP events are produced by brief, singular, isolated, intracloud electrical discharges that occur in intense regions of thunderstorms. These discharges have been dubbed CIDs, an acronym for compact intracloud discharges. During the summer of 1996, ground-based receiver arrays were used to record the electric field change signals and broadband HF emissions from hundreds of CIDs. Event timing that was accurate to within a few microseconds made possible the determination of source locations using methods of differential time of arrival. Ionospheric reflections of signals were recorded in addition to groundwave/line-of-sight signals and were used to determine accurate altitudes for the discharges. Twenty-four CIDs were recorded from three thunderstorms in the southwestern United States (US). The events occurred at altitudes between 8 and 11 $\mathrm{km}$ above mean sea level (MSL). Radar reflectivity data from two of the storms showed that CIDs occurred in close spatial proximity to thunderstorm cores with peak radar reflectivities of 47 to $58 \mathrm{dBZ}$. Over one hundred $\mathrm{CDs}$ were also recorded from tropical cyclone Fausto off the coast of Mexico. These events occurred at altitudes between 15 and $17 \mathrm{~km}$ MSL. CDs are singular discharges that usually occur in temporal isolation from other thunderstorm radio emissions on time scales of at least a few milliseconds. Calculations show that the discharges are vertically oriented and 300 to $1000 \mathrm{~m}$ in spatial extent. They produce average currents of several tens to a couple hundred kA for time periods of approximately $15 \mu \mathrm{s}$. Based on the results of a charge distribution model, the events occur in thunderstorm regions with charge densities on the order of several tens of $\mathrm{nC} / \mathrm{m}^{3}$ and peak electric fields that are greater than $1 \times 10^{6} \mathrm{~V} / \mathrm{m}$. Both of these values are an order of magnitude greater than values 
previously measured or inferred from in situ thunderstorm measurements. The unique radio emissions from $\mathrm{CDs}$, in combination with their unprecedented physical characteristics, clearly distinguish the events from other types of previously observed thunderstorm electrical processes. 


\section{ChAPTER 1}

\section{INTRODUCTION}

A small (113 kg) satellite named ALEXIS (Array of Low Energy X-ray Imaging Sensors) was launched on a Pegasus booster on 25 April 1993 into a $750 \times 850 \mathrm{~km}$, $70^{\circ}$ inclination orbit. ALEXIS was conceived of and built by Los Alamos National Laboratory (LANL) and carries two scientific payloads designed to test space-based technologies for detecting emissions from nuclear detonations (NUDETs). The primary payload, which carries the same name as the satellite, includes an array of six telescopes that collect ultrasoft X-ray emissions. The secondary payload, named Blackbeard, is a broadband VHF radio science experiment. Both payloads have made significant contributions to their programmatic missions and, in the process, have served as excellent platforms for unique astronomical and geophysical remote sensing research. ALEXIS has provided the first maps of celestial soft X-ray emissions. Blackbeard, from its perspective in low-earth orbit, discovered an entirely new and mysterious class of radio emissions from the earth. The distinct, paired emissions were dubbed transionospheric pulse pairs (TIPPs) by Holden et al. [1995], and have led to the identification of a unique type of thunderstorm electrical discharge. The discharges, which shall be referred to as compact intracloud discharges (CDs), are compact intracloud lightning events that occur in the most intense regions of 
thunderstorms. CIDs are distinct in many respects from other types of previously described thunderstorm electrical processes. These unique discharges are the subject of this dissertation.

\subsection{BLACKBEARD AND TRansIONOSPHERIC PULSE PAIRS (TIPPS)}

Difficulties were encountered during the launch of the ALEXIS satellite. Premature deployment of a solar panel caused damage to its magnetometer and prevented ground controllers from making contact with the satellite until late June of 1993, two months after its launch into low-earth orbit. Modified attitude control procedures were implemented to minimize problems associated with the launch anomaly and full satellite operations were begun in late July. Details regarding the satellite, payloads, and subsequent operations have been described by Priedhorsky et al. [1993] and Roussel-Dupré et al. [1997].

The Blackbeard instrument was designed to test technologies related to the spacebased detection and timing of electromagnetic pulses (EMPs) produced by nuclear weapons. The EMP from a nuclear explosion is a time-varying electromagnetic field that very rapidly (on the order of 10 nanoseconds) reaches it peak value and decays less quickly (during a few tens of microseconds) back to a negligible value. It is produced by asymmetries in the blast environment of a weapon that cause net timevarying currents to flow. Asymmetries may result from weapon design, proximity of the blast to the earth's surface, atmospheric air density gradients, or other 
environmental constraints. A description of the EMP and its effects was published by Glasstone and Dolan [1977]. The EMP is notorious for its ability to damage unprotected electronic devices. It also provides a means of detecting and accurately locating NUDETs. Such a capability is a valuable asset for the worldwide monitoring of nuclear activities and for verifying international compliance with treaties of nonproliferation.

EMPs are powerful radio emissions that radiate across a broad spectrum of frequencies from tens of $\mathrm{kHz}$ or lower to at least several hundred $\mathrm{MHz}$ (as indicated approximately by the inverse of the EMP rise time). For this reason Blackbeard was designed as a broadband receiver, providing in a sense, a receiver matched to the emission source it was designed to detect. Blackbeard operates in the VHF portion of the electromagnetic spectrum because lower frequency radio emissions (HF and below) often do not escape the refractive effects of the earth's ionosphere and thus, do not reach low-earth orbit. EMP radio emission frequencies greater than VHF are less powerful, so, more difficult to detect. The matter of detecting and time tagging broadband VHF signals from orbit is complicated by the dispersive and refractive effects of the ionosphere. These effects become increasingly severe at lower frequencies in proportion to wavelength squared.

The Blackbeard radio receiver records waveforms using a fixed-rate 150 Msample/s, 8-bit digitizer that takes its input from either of a pair of wideband subresonant monopole antennas and a single-conversion VHF receiver. There are two bands from which the instrument can sample: a low band from 28 to $95 \mathrm{MHz}$ and a high band from 108 to $166 \mathrm{MHz}$. Sixteen highpass and lowpass analog filters permit 
further subdivision of either band. The instrument utilizes a level trigger to detect transient events. Details concerning the Blackbeard instrument and subsequent data acquisition were described by Holden et al. [1995] and Massey and Holden [1995].

Prior to the launch of Blackbeard it was known that radio emissions from natural lightning produced transient broadband emissions in the VHF spectrum and would be a potential source of false alarms for any system designed to detect a nuclear EMP. In fact much of the motivation for orbiting the Blackbeard payload was provided by the need to characterize the earth's radio background. The characterization was necessary for both transient signals, like those produced by lightning, and CW (continuous wave) signals, like those emitted by commercial radio and television stations. Although Blackbeard provides a receiver well-matched to the detection of broadband transients, CW signals can still degrade its sensitivity when many, powerful carriers exist within its bandwidth. The detectability of transient signals in carrier-dominated radio environments was discussed by Smith [1995].

In November of 1993 the first Blackbeard geophysical study was begun. The goal of the study was to detect thunderstorm radio emissions over the equatorial intertropical convergence zone (ITCZ). Three months later, after determining optimal instrument gains, filter settings, and trigger thresholds, 85 events of subionospheric origin had been recorded. Two very remarkable and unexpected qualities characterized all of these events: 1 . Every Blackbeard record contained exactly two transient signals that were separated by between 10 and $110 \mu \mathrm{s} ; 2$. The RF power in the paired signals was on the order of a hundred times greater than that from lightning emissions described by previous researchers. The mysterious, paired VHF pulses 
were dubbed TIPP events by Holden et al. [1995]. To date, over 1100 of the events have been recorded by Blackbeard (Dorothea DeLapp, private communication). An example of a TIPP electric field waveform from Blackbeard is shown in the upper panel of Figure 1.1. A time-frequency spectrogram of the waveform appears in the lower panel. The spectrogram shows the power in the signal as a function of frequency and time. It was formed by dividing the time series into a number of short, overlapping time series that were processed using the fast Fourier transform to determine short term power spectra. The resulting spectra were aligned vertically and a color scale was utilized to represent localized waveform log power. The spectrogram in Figure 1.1 was formed using a 128 point ( 850 ns) sliding Blackman window. Successive waveform segments were overlapped by $50 \%$. The TIPP event in the spectrogram is the pair of powerful, dispersed transients. The VHF pulses are separated in time by approximately $60 \mu$ s. Dispersion of the signals was caused by propagation through the ionosphere. The dim, horizontal lines in the figure are narrowband carrier signals, most likely from ground-based transmitters (ALEXIS was over central Africa when it recorded the event). The vertical lines that are most clearly visible at lower frequencies (below $35 \mathrm{MHz}$ ) were probably produced by the satellite itself, as suggested by their lack of dispersion and their frequent appearance in Blackbeard records.

Based on Blackbeard observations, TIPPs have the following general characteristics: By definition they are paired. Triggered records that contain pairs have greatly outnumbered (by a ratio greater than 10:1 as estimated by the this author) records that contain singular pulses or pulses with multiplicity greater than two. As 
Time Series for e 11217
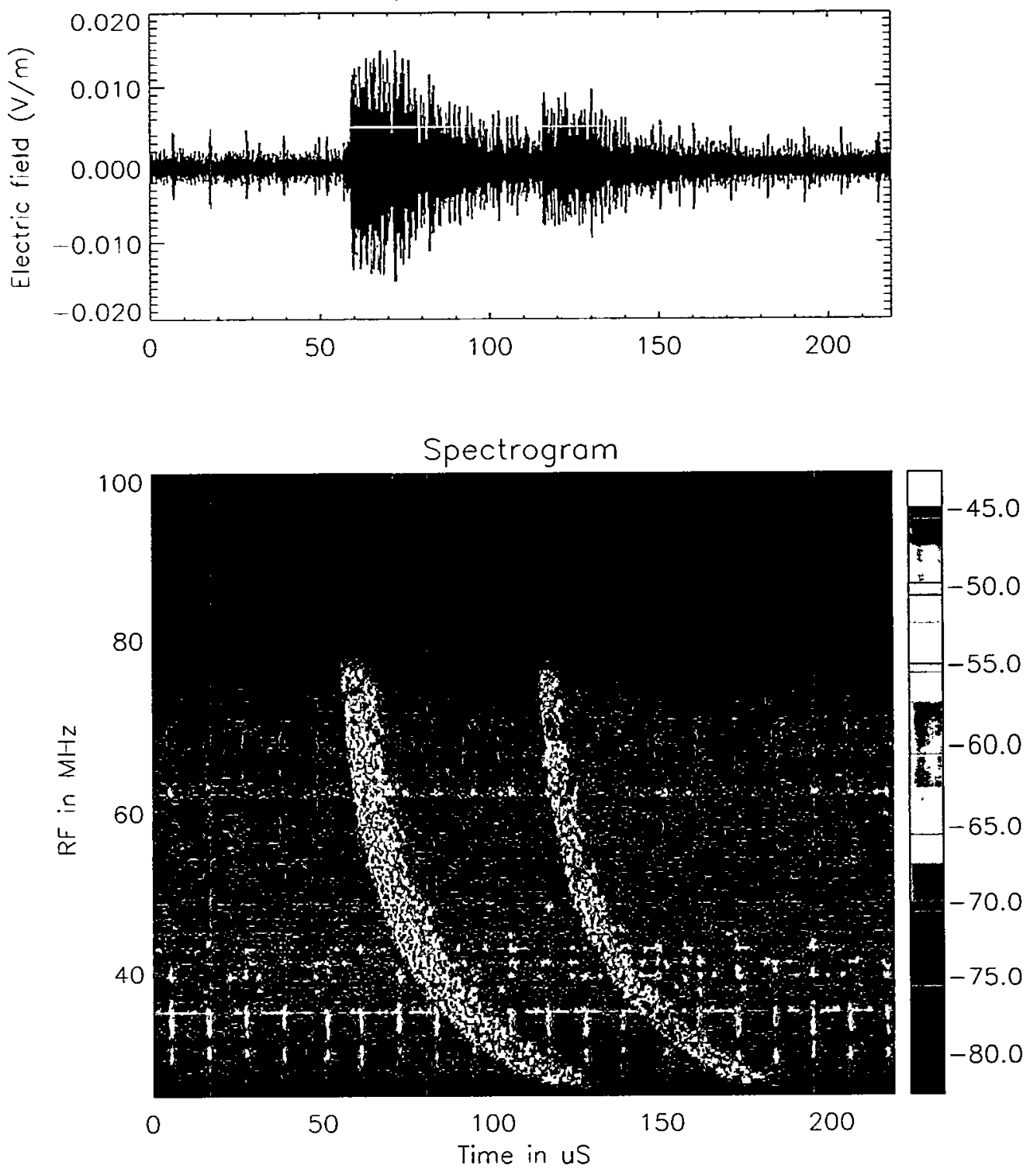

Figure 1.1: Electric field waveform and spectrogram of a transionospheric pulse pair (TIPP) event that was recorded by the Blackbeard instrument over Africa. The spectrogram color scale represents waveform power in $\mathrm{dB}$ as a function of time and frequency. 
reported by Massey and Holden [1995], the mean duration for each pulse is $5 \mu$ s and the median duration is $3.8 \mu$ s (after the dispersive effects of the ionosphere have been removed by signal processing). Observed pulse separations have ranged from a few microseconds to greater than $100 \mu \mathrm{s}$, with the mean and median separations both being very close to $50 \mu \mathrm{s}$ [Massey and Holden, 1995]. The low end of this distribution is difficult to characterize and interpret accurately because the two pulses become indistinguishable at a minimum separation that is on the order of the duration of each pulse. TIPPs radiate very strongly across the entire recording range of Blackbeard from 28 to $166 \mathrm{MHz}$. TIPP peak electric field values are about ten times greater than lightning peak electric fields that have been observed during groundbased studies of lightning radio emissions [Holden et al., 1995]. It has been observed that TIPP events are most often recorded over locations and at times of day where and when thunderstorm activity is known to be most active [Holden et al., 1995; Zuelsdorf et al., 1997].

The most striking characteristic of TIPP recordings has been the fact that dual pulses have been recorded so much more frequently than pulses of other multiplicity. There is scant specific mention of double pulses in the literature describing previous observations of radio emissions from lightning. The observed time separations between the pulses (10 to $110 \mu$ s as reported by Holden et al. [1995]) are not inconsistent with previous reports of the inter-pulse spacing between RF (radio frequency) pulses during lightning flashes, but "normal" discharges most often consist of thousands to tens of thousands of pulses that are radiated during a flash that lasts a 
few to many tenths of a second [Oetzel and Pierce, 1969, a; Proctor, 1973; Pierce, 1977]. The corresponding inter-pulse intervals are on the order of tens to hundreds of microseconds, but reports of pulses occurring with a multiplicity of exactly two have been scarce. Two hypotheses were proposed to explain why, in the overwhelming majority of Blackbeard recordings, paired pulses were received. The explanations, which shall be referred to as the one-source and two-source hypotheses, were introduced by Holden et al. [1995] and elaborated upon by Massey and Holden [1995], Smith [1995], Smith and Holden [1996], and Massey et al. [1998]. The onesource hypothesis considers the source of a TIPP to be a singular discharge that reflects with little loss from the surface of the earth. The reflection is recorded by Blackbeard as the second pulse. The two-source hypothesis considers a TIPPs to result from two coupled discharges that occur at different times and/or in different locations. Figure 1.2 provides illustrations of the two hypotheses.

\subsubsection{One-Source Hypothesis}

Under the one-source hypothesis, the second pulse of a TIPP is the reflection of downward-directed source emissions from the surface of the earth. This possibility is illustrated in the upper panel of Figure 1.2. The measured time separation between the direct pulse and its reflection is a function of the height of the source above ground level and the position of the receiver with respect to the source. An approximate relationship between pulse time separation $(\Delta t)$, source height $(h)$, and satellite elevation angle $(\theta)$ is given by Equation 1.1: 

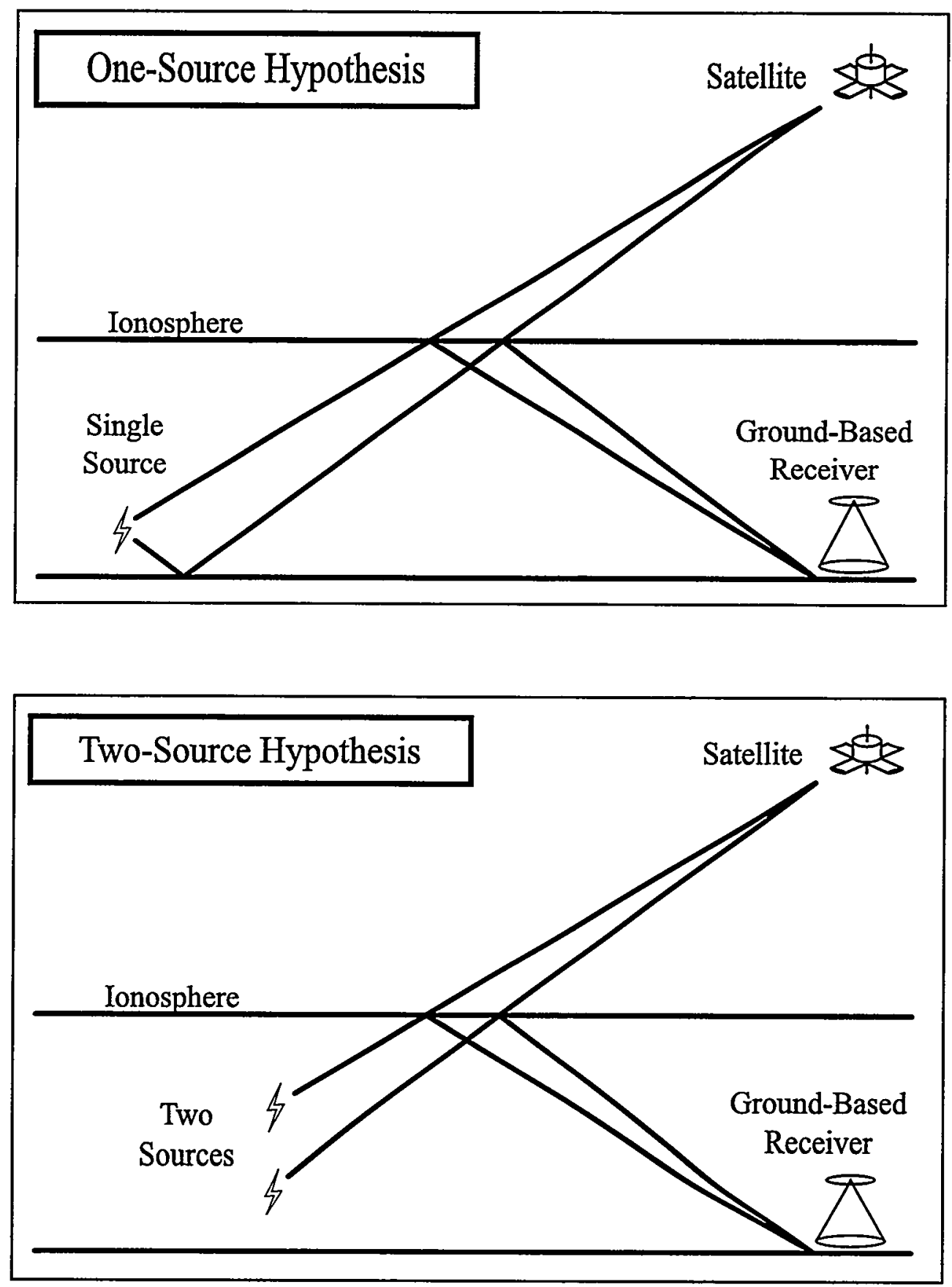

Figure 1.2: Illustrations of the one-source and two-source hypotheses for the production of TIPP (transionospheric pulse pair) and SIPP (subionospheric pulse pair) events. 


$$
\Delta t=\frac{2 h \sin (\theta)}{c}
$$

where $c$ is the speed of light. This equation is approximately true for cases when the satellite altitude $(H)$ is much greater than the source altitude (i.e. $H \gg h$ ).

Figure 1.3 illustrates the source and satellite geometry for the case described by Equation 1.1. Figure 1.4 shows the actual relationship between elevation angle and pulse separation for a source at a given height. The top graph shows pulse separation as a function of elevation angle for a source at an altitude of $10 \mathrm{~km}$ above ground level (AGL). From zenith, the satellite measures a separation of $67 \mu \mathrm{s}$. From the $\operatorname{limb}\left(\theta=0^{\circ}\right)$, the pulses are indistinguishable $(\Delta t=0 \mu \mathrm{s})$. The bottom graph shows pulse separation normalized to the height of the source above ground $(h)$. Note that at zenith $\left(\theta=90^{\circ}\right)$, the observed normalized pulse separation is 2.0 . This means that the delay from the time of arrival (TOA) of the direct pulse to the TOA of the reflected pulse is equal to exactly two times the source height divided by the propagation velocity. This makes sense, because for the reflected pulse to be received, it must propagate straight down and back through the source origin, traveling an additional path length $2 h$ compared to the direct pulse.

Equation 1.1 and Figures 1.3 and 1.4 show that the maximal time separation between pulses for a given source height occurs when the satellite is positioned directly above the source. Short time separations can occur from sources that are located near ground level (small $h$ ) or sources that occur near the horizon (small $\theta$ ) as viewed from the satellite receiver. 


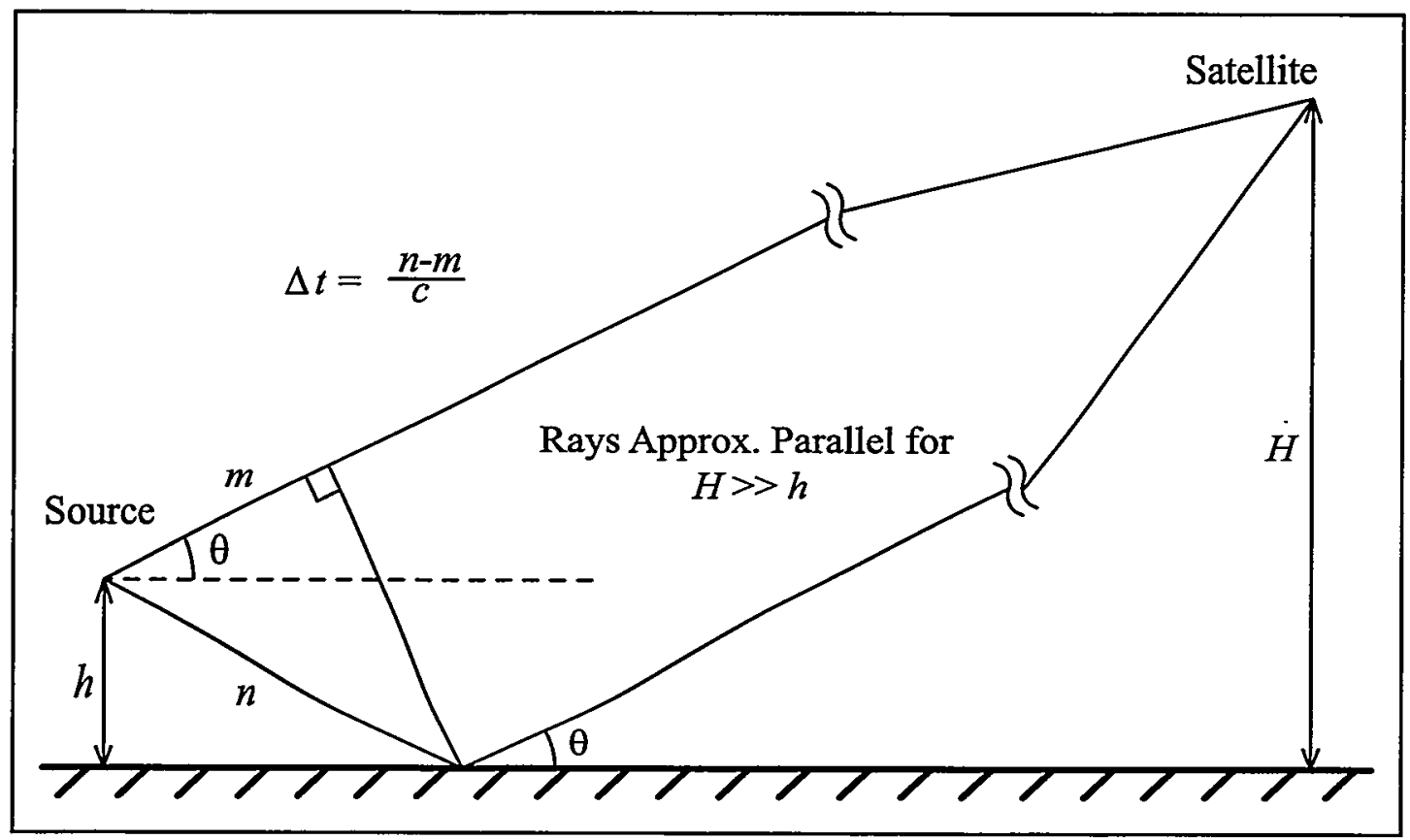

Figure 1.3: Illustration of the reflection geometry for the one-source hypothesis. The direct and reflected rays can be assumed to be parallel for $H \gg h$, under which condition the pulse time separation $(\Delta t)$ is a function of the source height $(h)$ and satellite elevation angle $(\theta)$ only. 

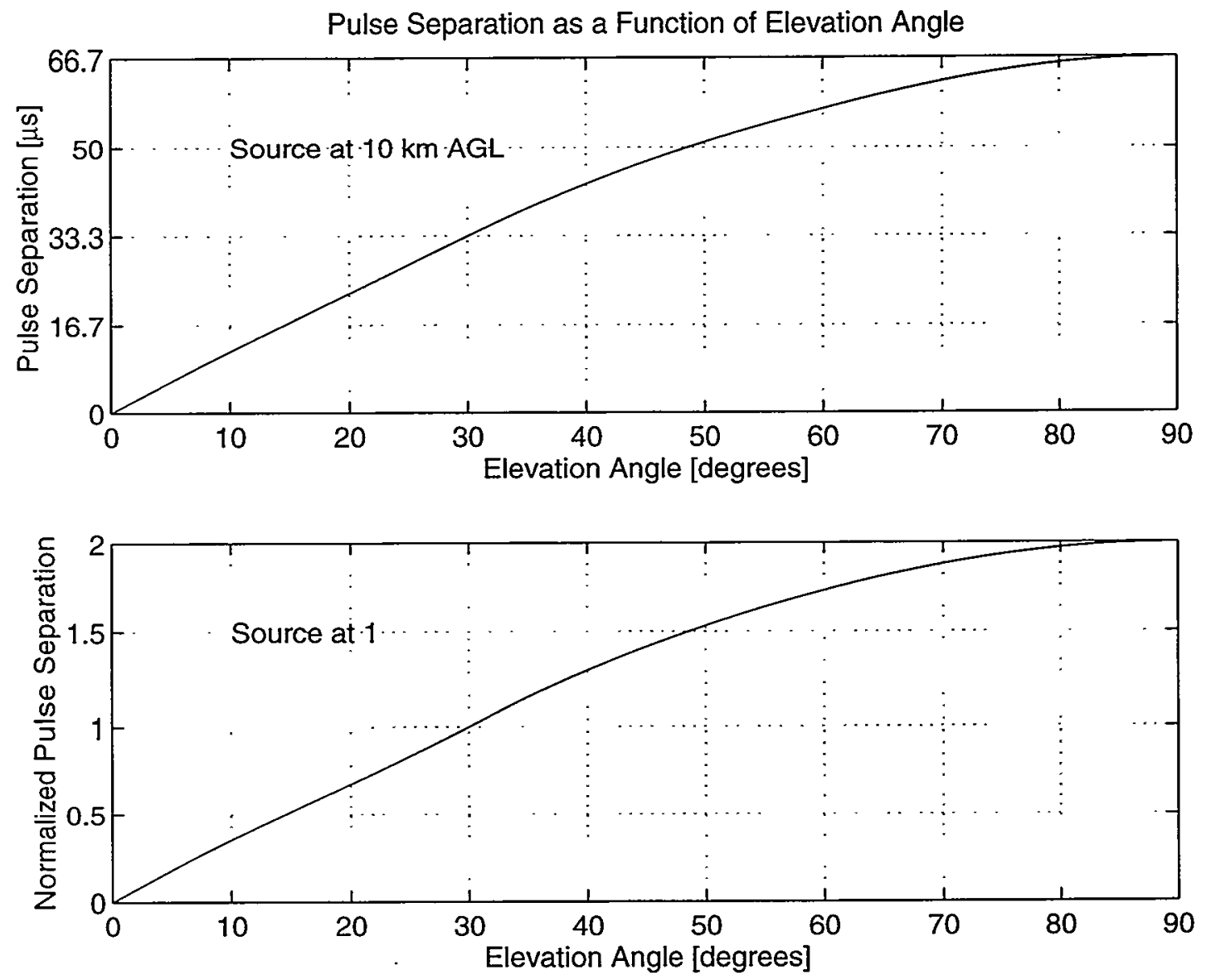

Figure 1.4: Plots of pulse separation as a function of elevation angle for sources at a fixed height. In the upper panel, the source height is $10 \mathrm{~km}$ above ground level (AGL) and pulse separation is given in microseconds. In the lower panel, pulse separation is normalized to a source height of 1 (arbitrary units). 
The actual distribution of pulse separations for the first 84 TIPP events recorded by Blackbeard is shown in Figure 1.5 (adapted from Massey and Holden [1995]). Note that four events with time separations of less than $10 \mu$ s were received. Under the one source hypothesis, these events either occurred close to the earth's surface or were viewed from a shallow viewing angle by Blackbeard (or both).

At the far right end of the distribution in Figure 1.5 are TIPPs that had pulse separations as large as $100 \mu \mathrm{s}$. These events place a lower bound on the upper limit of source heights. That is, for a given measured pulse pair separation, the actual event source height must have been at least that which results from inverting Equation 1.1 and solving for $h$ when the source is assumed to have occurred with the satellite at zenith $\left(\theta=90^{\circ}\right)$. For example, the largest pulse separation for a TIPP event recorded by Blackbeard has been approximately $120 \mu$ s (the event occurred after the distribution depicted in Figure 1.5 was formed). Under the one-source hypothesis, the height of the source would have had to have been at least $18 \mathrm{~km}$ above ground level, the value for $h$ calculated from Equation 1.1 for $\theta=90^{\circ}$ and $\Delta t=120 \mu \mathrm{s}$. The further from Blackbeard's nadir that source actually occurred, the higher in altitude the source would have had to have been in order to produce the measured time separation. Thus large time separations place a lower bound on the upper limit of the actual source height distribution. If the one-source hypothesis is correct, then the mechanism for the production of the singular discharge must allow for the possibility that the events can occur up to an altitude of at least $18 \mathrm{~km}$ above ground level. 


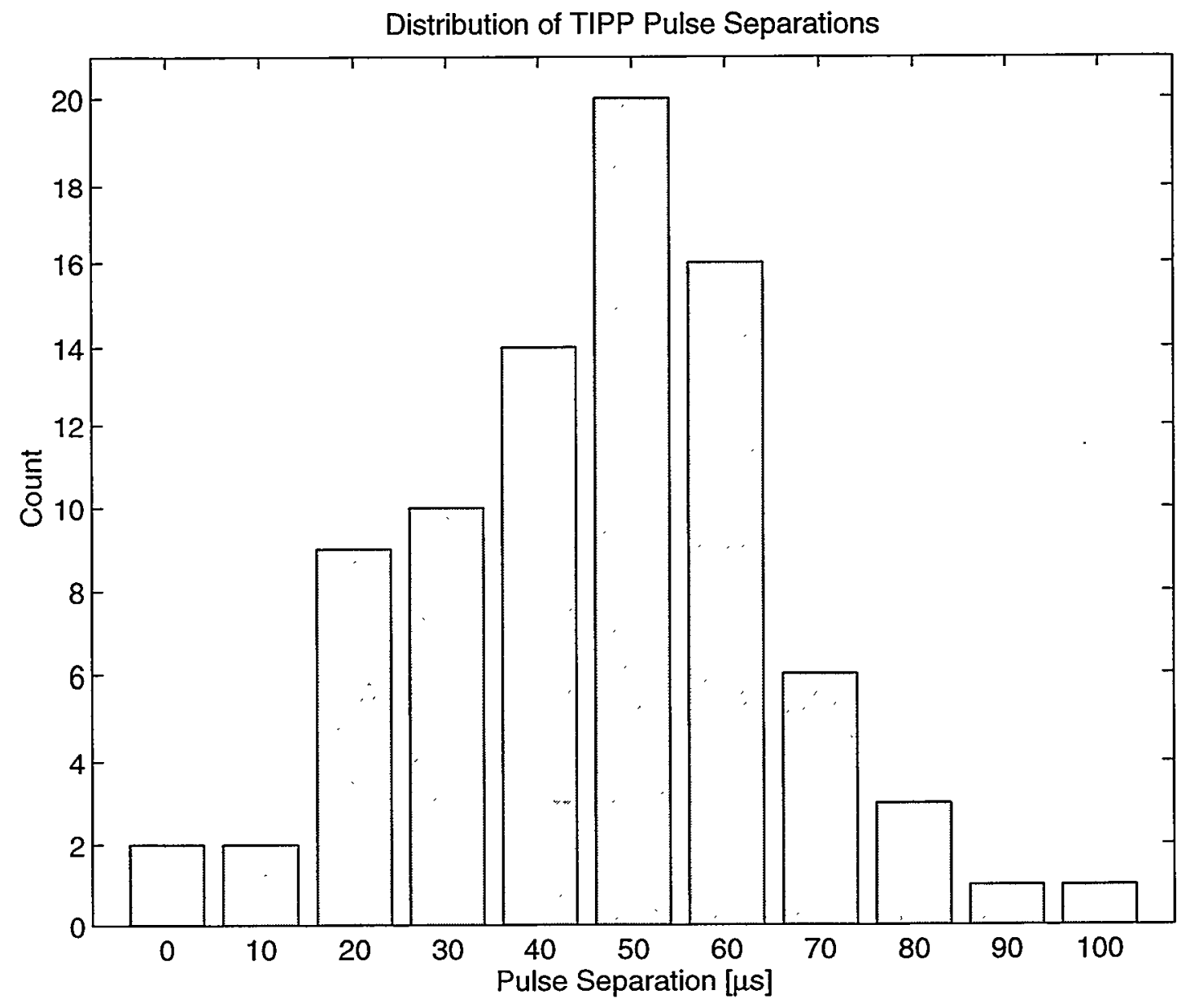

Figure 1.5: Distribution of pulse separations for 84 early TIPP events that were recorded by Blackbeard and analyzed by Massey and Holden [1995]. 
Equation 1.1 and Figure 1.4 are valid only for the case when $H \gg h$. A model presented by Massey and Holden [1995] showed that the distribution of pulse separations in Figure 1.5 is consistent with the typical TIPP source altitude being on the order of $15 \mathrm{~km}$. This result is consistent with associations between TIPP events and thunderstorm activity [Holden et al., 1995; Smith, 1995; Smith and Holden, 1996; Zuelsdorf et al., 1997], since thunderstorms are tropospheric phenomena and the tropopause may extend as high as $20 \mathrm{~km}$ in the tropics. The fact that TIPPs are produced within a couple tens of kilometers of the surface of the earth means that the assumption that $H \gg h$ is always true for these events, since $\mathrm{H}$ is always approximately $800 \mathrm{~km}$.

The one-source hypothesis also imposes requirements regarding the radiation pattern of the source and the reflectivity characteristics of the earth's surface at VHF frequencies. In order for the hypothesis to be valid, the following conditions must be met:

Firstly, the radiation pattern of the source must be broad in the vertical plane. This is so because the direct path and reflected path leave the source at quite different angles for events that occur with the satellite at a high elevation angle. For cases when the source lies close to the limb of the earth as viewed from Blackbeard, the angle between the direct and reflected paths is quite acute, but the broad distribution of time separations effectively rules out the possibility that this is always the case if TIPPs are produced in the troposphere.

Secondly, the typical surface reflectivity of the earth must be high (near unity) and reflections must be nearly specular in nature at VHF frequencies [Massey and 
Holden, 1995; Massey et al., 1998]. These conditions must be met because TIPP second pulses contain, on average, almost as much energy as first pulses. In fact, of the first 84 low band TIPPs recorded, the second pulse actually had more energy than the first in 23 of the cases [Massey and Holden, 1995]. Thus if the one-source hypothesis is true, little energy can typically be lost at the earth reflection interface. It is also necessary to explain how it is possible for reflected pulses to have more energy than direct pulses when second pulses are subject to greater range loss (although only $0.8 \mathrm{~dB}$ for the worst-case scenario of a source at an altitude of $20 \mathrm{~km}$ and the satellite directly overhead at an altitude of $800 \mathrm{~km}$ ) as well as reflection loss (although only $1.1 \mathrm{~dB}$ for poor soil conditions as concluded by Massey et al. [1998]). One possible explanation is that for some fraction of the events, the geometry of the reflection point may enhance the reflected signal. This could occur when mountains in the vicinity of the earth reflection point provided a greater reflecting area. A second explanation is that, depending on satellite/source geometry and the radiation pattern of the source, some fraction of the time the direct path to the satellite may be closer to a null in the source radiation pattern than the earth-reflected path.

Recent experimental results from Massey et al. [1998] suggest that the earth reflectivity requirements are met, even by the dry and sandy desert soil that was present where their experiment was carried out. Sandy soil is a worst case scenario, since its conductivity is low compared to other soils and much lower than sea water, which blankets most of the earth. 


\subsubsection{Two-Source Hypothesis}

Under the two-source hypothesis the two TIPP emissions are assumed to be produced by two different, yet coupled, sources that are separated temporally and/or spatially. The lower panel of Figure 1.2 provides an illustration of this hypothesis. In order for the hypothesis to be consistent with Blackbeard data and an apparent lack of previous ground-based observations of powerful, paired pulses, the radiation pattern of the source must be directed upward. This is so for two reasons: 1. Strong pulse pairs have been absent from ground-based observations of high frequency emissions from lightning. If strong dual pulses are produced regularly by thunderstorms, then they would have most likely been observed from the ground, unless directed upward. 2. If TIPP second pulses are considered to be from a second source, then it must be questioned why reflections are not received. Either the source radiates primarily upward or the reflectivity of the earth is poor. Measurements by Massey et al. [1998] showed that the latter possibility is not true.

A theory describing a potential source for upward-propagating, paired radio flashes was published by Roussel-Dupré and Gurevich [1996]. In the theory, electrons with energies greater than a critical value are accelerated to high energies by thunderstorm electric fields that produce electrical forces greater than the frictional force of air. Impact ionization of the air by the energetic electrons leads to the production of energetic secondary electrons, whose energies also exceed the critical value. The net result is an avalanche in which the electron population grows exponentially. Collimation of the relativistic electrons by the electric field leads to 
the formation of an electron beam. Signatures of this process would include optical, $\gamma$-ray, $\mathrm{x}$-ray, and radio emissions. The radio emissions result from the acceleration and deceleration of the primary and secondary electron populations. Due to the relativistic nature of the process, the radio bursts are emitted primarily along an upward-directed cone. The angle of the cone from zenith depends on factors like the field strength, the number of avalanche lengths, and the air pressure, but is on the order of $15^{\circ}$. The runaway electron theory also has the potential for explaining observations of luminous flashes known as sprites and jets, which have been observed above thunderstorms [Sentman et al., 1995; Wescott et al., 1995]. It could also explain observations of $\mathrm{x}$-ray pulses by instrumented balloons in thunderstorms [Eack et al., 1996, a; Eack et al., 1996, b] and observations of intense $\gamma$-ray flashes by the BATSE (Burst and Transient Signal Experiment) instrument on board the Compton Gamma Ray Observatory (CGRO) [Fishman et al., 1994].

Observations and analyses presented in this paper will show that the one-source hypothesis is consistent with both Blackbeard and ground-based observations of radio signals from the source of TIPP events. The two-source hypothesis is not consistent with recent ground-based observations. The runaway electron theory by RousselDupré and Gurevich [1996], however, still has the potential for explaining CIDs, the discharges that produce TIPP events (Robert Roussel-Dupré, private communication). 


\subsection{SUBIONOSPHERIC PULSE PAIRS (SIPPS)}

Following the discovery of TIPP events in 1993 by Blackbeard, ground-based research campaigns were begun at Los Alamos National Laboratory to try to detect and characterize the events from terra firma. During the summer of 1994, a broadband data acquisition system was used to record signals over a frequency range from 3 to $30 \mathrm{MHz}$ using a discone antenna. A discone antenna consists of a grounded cone, topped by a disc that serves as the receiving element. Data were digitized at 50 or $100 \mathrm{Msample/s}$ after passing through a $30 \mathrm{MHz}$ lowpass filter (at the $50 \mathrm{Msample/s}$ sample rate, aliasing of the 25 to $30 \mathrm{MHz}$ input signal to the 20 to $25 \mathrm{MHz}$ frequency band occurred). The trigger for the digitizer was a specialized multiple-channel subband trigger that detected broadband transient signals against a background dominated by high-power $\mathrm{CW}$ signals. The instrumentation and subsequent observations were described by Smith [1995] and Smith and Holden [1996].

During the summer thunderstorm season, two classes of paired HF emissions were recorded and identified as candidates for the ground-recorded emissions from TIPP events. The two emission types were collectively dubbed subionospheric pulse pairs (SIPPs). One class of SIPPs was ionospherically dispersed, indicating propagation from over the horizon. The other class was undispersed, indicating lineof-sight propagation. 


\subsubsection{Dispersed Subionospheric Pulse Pairs}

During the summer of 1994, seven dispersed SIPPs were recorded from over the horizon by the ground-based LANL broadband HF data acquisition system. The observations of obliquely propagated pulse pairs closely resembled Blackbeard observations of TIPP events in the following respects: the events occurred as pairs, were very powerful, and were temporally isolated from other transient RF signals. Two of the events were analyzed in detail and provided further evidence of an association between pulse pair events and thunderstorm activity [Smith, 1995; Smith and Holden, 1996].

An example of one of the events is shown in Figure 1.6. Two dispersed pulses are visible between 13 and $25 \mathrm{MHz}$ against a background of multiple narrowband communication signals. The pulses were separated by 24 microseconds and demonstrated competing time-frequency effects that often characterize obliquelypropagated HF signals (a description of these effects was provided in Appendix B of Smith [1995]). The signal in Figure 1.6 was recorded within one minute of the signal in Figure 1.7, an emission with nearly identical dispersion characteristics, but which otherwise resembles HF radiation from a typical lightning streamer process. It was concluded that the similarity between the time-frequency properties of the events pictured in Figures 1.6 and 1.7 suggested that the two events originated in close proximity to each other. That the first emission was almost certainly from lightning indicates that the second emission, the pulse pair, originated in the vicinity of a thunderstorm. 


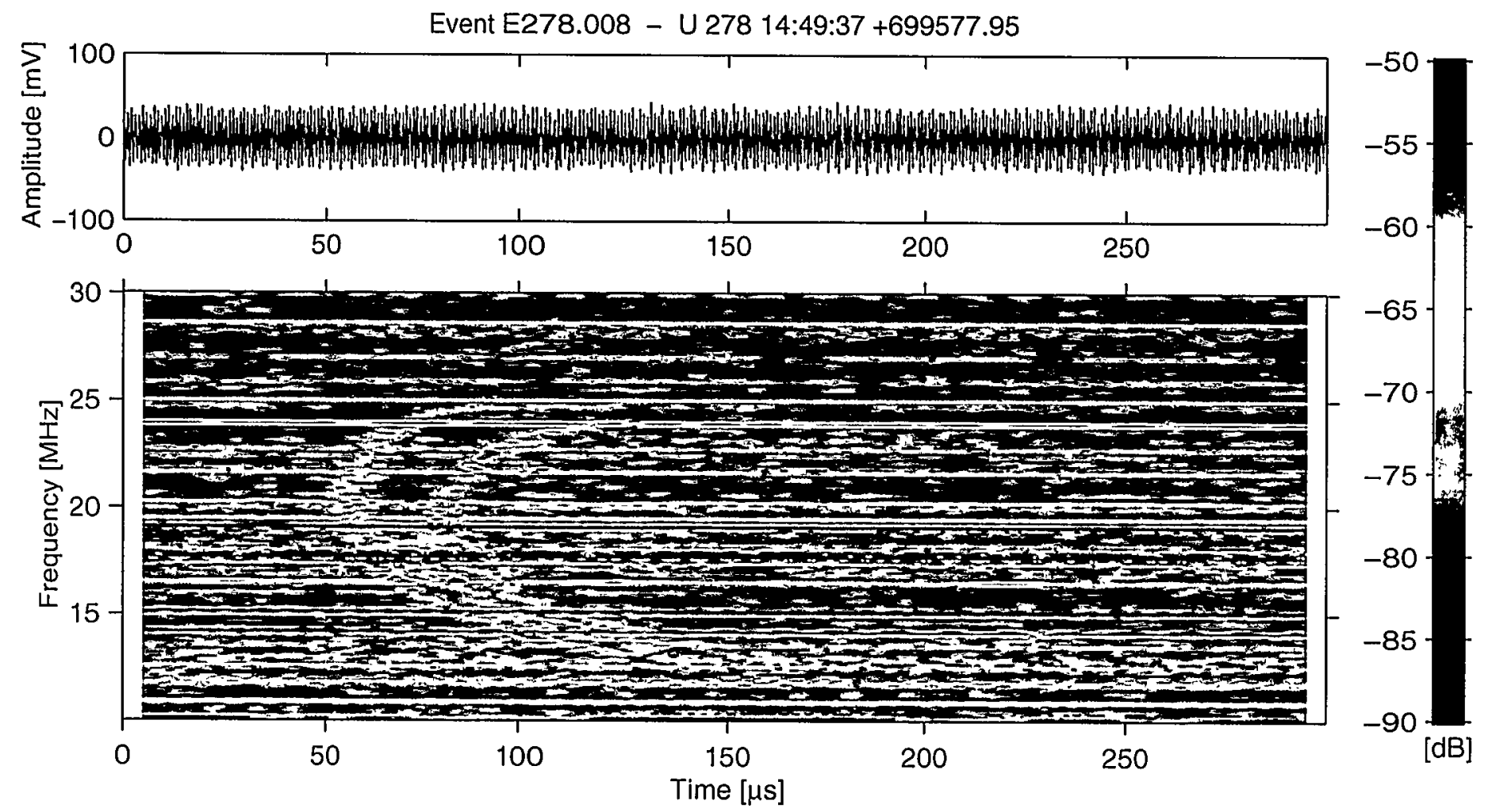

Figure 1.6: Time series and spectrogram for a subionospheric pulse pair (SIPP) that was recorded by the LANL ground-based broadband HF system during the summer of 1994. The dispersion of the two pulses indicate that they originated over the horizon and reflected from the ionosphere. 

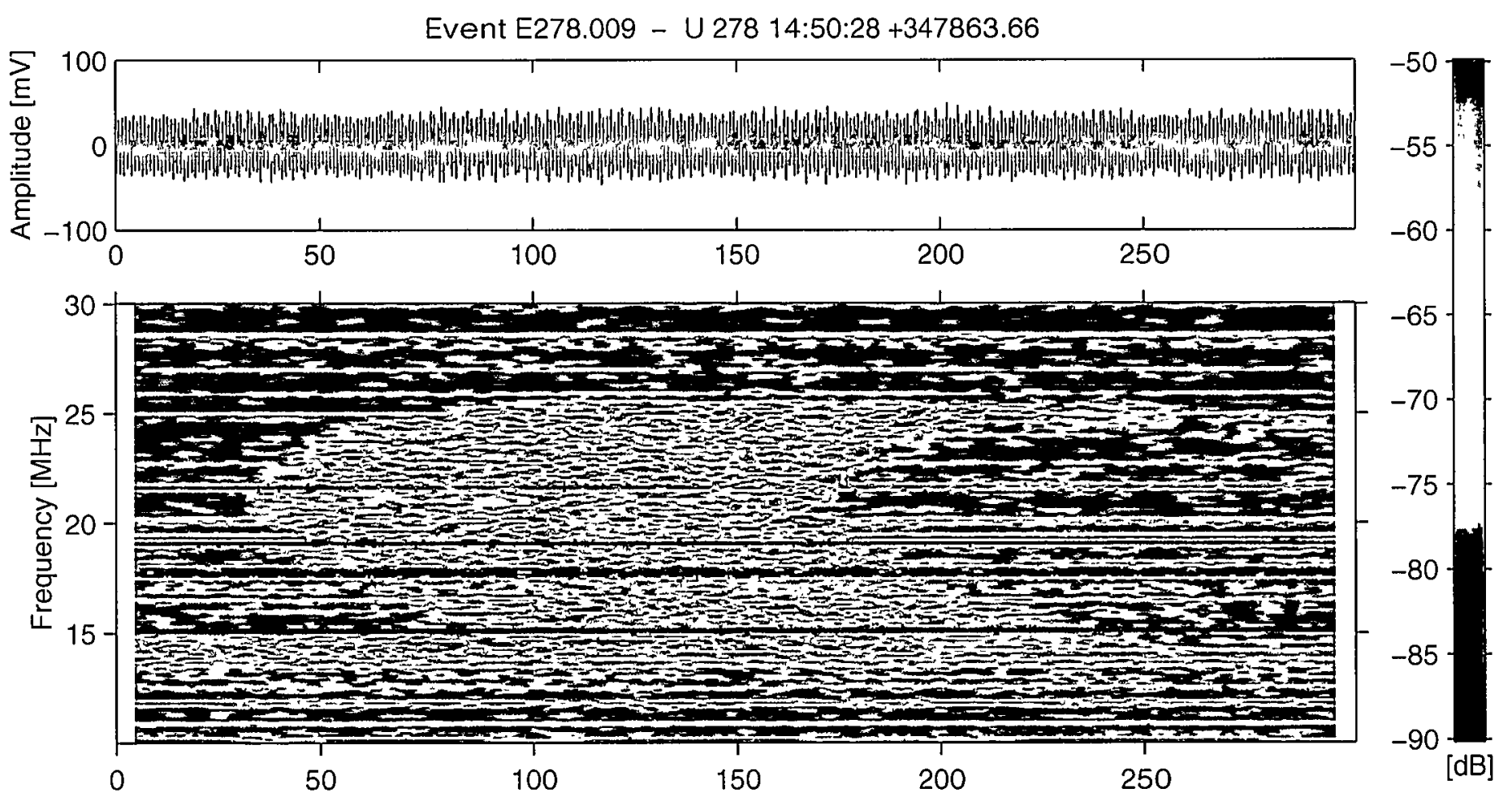

Figure 1.7: Time series and spectrogram for a dispersed burst of broadband radiation that was recorded by the LANL ground-based HF system during the summer of 1994 . The source is believed to have been distant lightning. 


\subsubsection{Undispersed Subionospheric Pulse Pairs}

Smith [1995] and Smith and Holden [1996] also described observations of undispersed SIPPs, pulse pairs for which all frequency components of the transient signals arrived at the receiver at the same time. An example of one of these events is shown in Figure 1.8. The time separation between the pulses was $22 \mu \mathrm{s}$. The lack of dispersion indicates that the signals propagated from their source or sources to the receiver along line-of-sight propagation paths. The maximum line-of-sight reception range for HF signals is a function of the source and receiver heights. The LANL HF systems can make line-of-sight observations of thunderstorms from distances up to around $350 \mathrm{~km}$.

It was stated by Smith [1995] and Smith and Holden [1996] that a lack of information about the locations of the sources of the undispersed pulse pairs made it impossible to compare their absolute signal strengths to those of TIPP events. This case was unlike that for the dispersed events because the fact that they were ionospherically dispersed guaranteed that the signals had distant origins. The authors stated that it was possible that the undispersed events actually had close origins and only appeared powerful as a result of their close proximity to the recording antenna. This author determined that this probably was the case, based on measurements made during the summer of 1997. A local anthropogenic RF emitter (a sodium lamp outside of the data acquisition lab) was identified as a source of intermittent nighttime radio pulse pairs. The discovery of the local source means that the observations of undispersed pulses pairs by Smith [1995] and Smith and Holden [1996] did not 

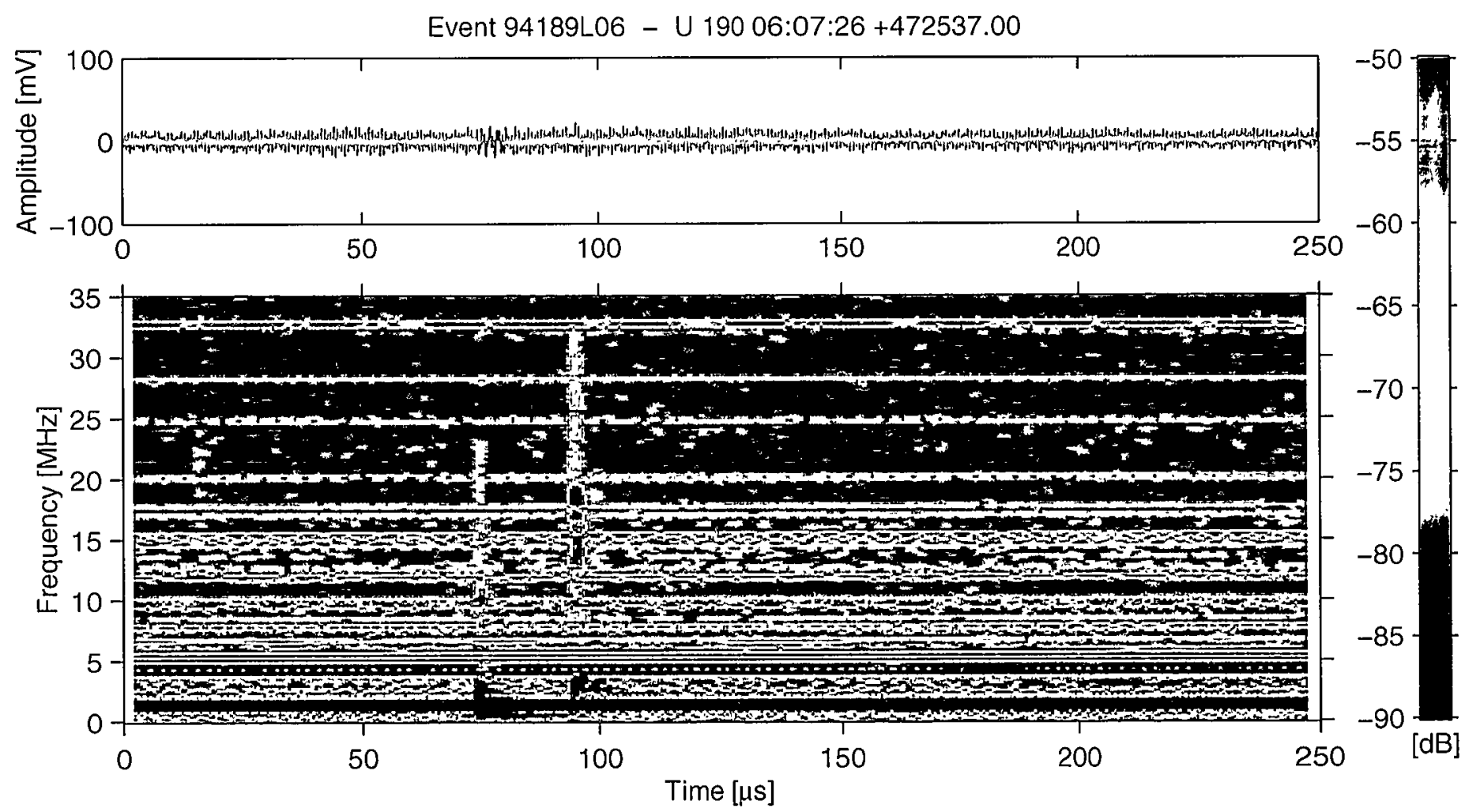

Figure 1.8: Time series and spectrogram for an undispersed subionospheric pulse pair that was recorded by the LANL ground-based HF system during the summer of 1994. 
provide insight into TIPP phenomenology. The observations had provided support for the two-source hypothesis over the one-source hypothesis, since a ground-based receiver would receive the direct and earth-reflected pulses from a single source within nanoseconds of each other, not tens of microseconds.

\subsection{NARROW POSITIVE BIPOLAR PULSES (NPBPS)}

In the summer of 1996 thunderstorm observations were continued. Two somewhat independent research campaigns were begun at LANL. The ground-based HF system was expanded to three stations (separated by 6 to $13 \mathrm{~km}$ ) so that events recorded by the three stations could be located using methods of differential time of arrival. The three stations were armed in conjunction with Blackbeard satellite passes to attempt to make simultaneous observations of TIPP events. The second campaign was conducted in cooperation with the New Mexico Institute of Mining and Technology (NMIMT). An array of three electric field change meters (separated by 30 to $230 \mathrm{~km}$ ) was operated to detect, identify, and locate field change emissions from sprites.

During simultaneous operations of the two arrays in July of 1996, it was observed that occasional, narrow, large amplitude, bipolar electric field change pulses were recorded in conjunction with very powerful, broadband $\mathrm{HF}$ radiation. At the time, the HF systems were receiving trigger signals from one of the field change meters. The $\mathrm{HF}$ and field change pulses were almost always isolated from other transient radio 
signals within their $1 \mathrm{~ms}$ and $5 \mathrm{~ms}$ respective records lengths. The signals were so distinct from other lightning emissions that, if not detected by multiple, widelyseparated recording stations, the signals could easily have been mistaken as noise from a local transient source. The HF emissions from the source were exactly what was expected from the source of TIPP events under the one-source hypothesis: the RF bursts were singular, isolated, brief (lasting a few microseconds), broadband, and much more powerful than lightning signals emitted from the same storms. The sharp, isolated, bipolar field change pulses that were observed were similar to pulses previously observed by Le Vine [1980], Willett et al. [1989], and Medelius et al. [1991]. Following the first observations of these signals, simultaneous operations of the electric field change and HF arrays were begun.

Le Vine [1980] identified the "sources of the strongest RF radiation from lightning" as thunderstorm cloud processes consistently recorded in conjunction with distinct, short-duration (10-20 $\mu$ s overall), bipolar electric field change pulses. Le Vine [1980] used three narrowband receivers with center frequencies of 3, 139, and $295 \mathrm{MHz}$ to trigger the acquisition of field change waveforms from an electric field change meter. When the trigger level of any of the RF receivers was set to a sufficiently high threshold during observations of thunderstorms, the corresponding electric field change waveforms were consistently isolated, short-duration, bipolar pulses. An example of a bipolar pulses from Le Vine [1980] is shown in Figure 1.9. Note that the polarity convention in the figure is opposite to that used throughout the remainder of this paper. Thus the pulse shown in Figure 1.9 is inverted compared to otherwise similar pulses presented here. 


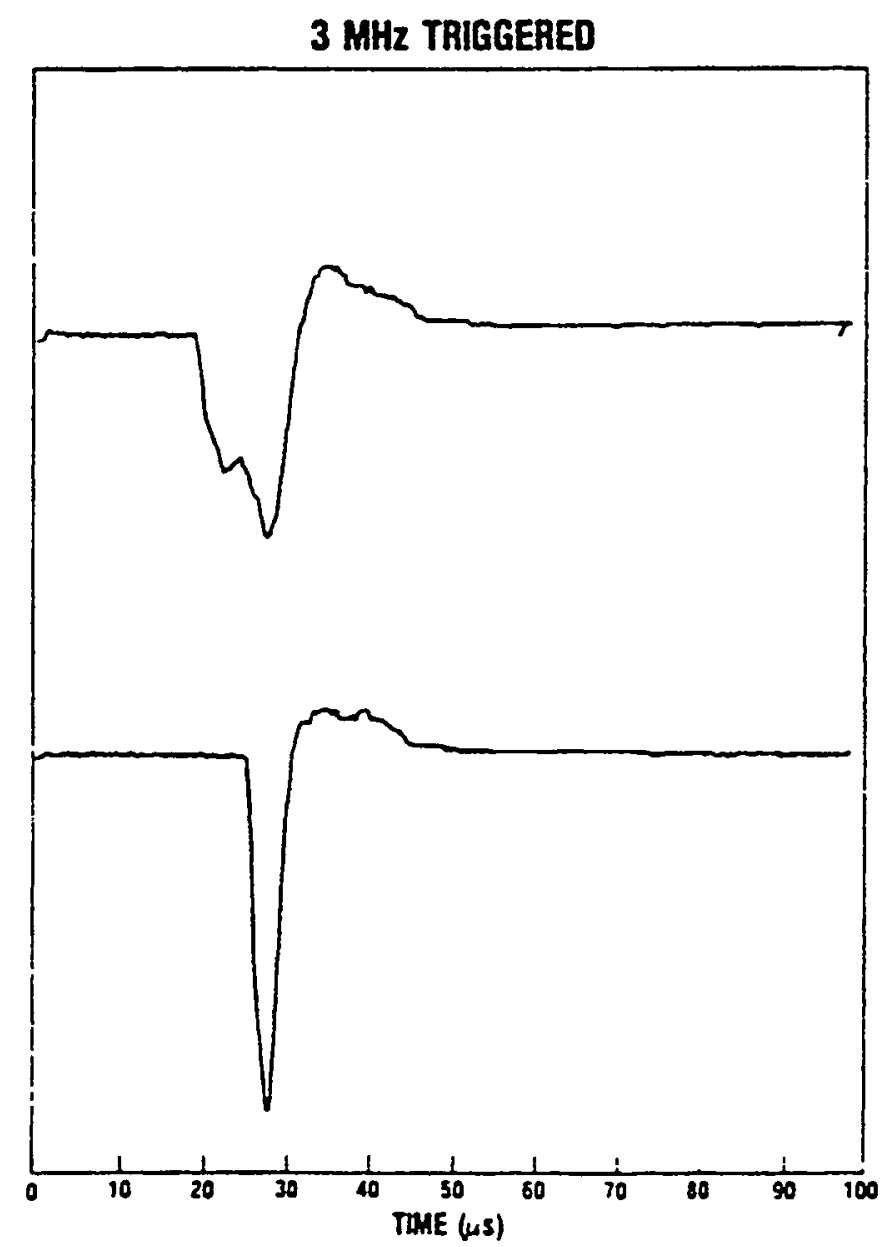

Fig. 2. Electric field changes tnggered on RF radiation at $3 \mathrm{MHz}$ (vertical polarization). The vertical scale is linear in Vim but uncalibrated. The direction of negatuve field change is down.

Figure 1.9: A narrow positive bipolar electric field change pulse recorded by LeVine [1980]. The field change meter was triggered by narrowband $\mathrm{RF}$ radiation centered at $3 \mathrm{MHz}$. The author used the opposite convention for electric field change polarity from that used throughout this dissertation. From D. M. Le Vine, Journal of Geophysical Research, 86, p. 4092, 1980, copyright by the American Geophysical Union. 
Willett et al. [1989] made observations consistent with those of Le Vine [1980] using a large bandwidth (greater than $30 \mathrm{MHz}$ ) fast electric field change meter. The distinct, isolated, bipolar waveforms were emitted from thunderstorms that also produced normal lightning activity. An example of one of their waveforms is shown in Figure 1.10. The bipolar waveforms recorded by Willett et al. [1989] had a mean full width at half maximum (FWHM) of $2 \mu \mathrm{s}$, overall durations of 20-30 $\mu$ s and peak amplitudes that were typically 0.72 times those from return strokes. Willett et al. [1989] dubbed the distinct field change waveforms narrow positive bipolar pulses (NPBPs). "Positive" refers to the initial polarity of the bipolar waveforms using the convention that a positive electric field change signal results from the deposition of negative charge overhead. Willett et al. [1989] also observed a few narrow negative bipolar pulses (NNBPs). The NNBPs were inverted in polarity, but otherwise similar to NPBPs in most respects. The authors concluded that "NPBPs are not usually associated with cloud-to-ground flashes, $\mathrm{K}$ changes in intracloud flashes, or other identified lightning processes." They additionally concluded that "it is not obvious how any of the customary models of electromagnetic radiation from lightning could be credibly modified to produce such $d E / d t$ signatures."

Medelius et al. [1991] made wideband electric field and electric field change measurements of lightning at the Kennedy Space Center in 1989. They identified and characterized over 150 short-duration bipolar pulses with characteristics that closely matched the observations of Le Vine [1980] and Willett et al. [1989]. Pulses of both positive and negative polarity were observed with negative pulses being detected ten times more frequently than positive pulses. FWHM durations were on the order of a 


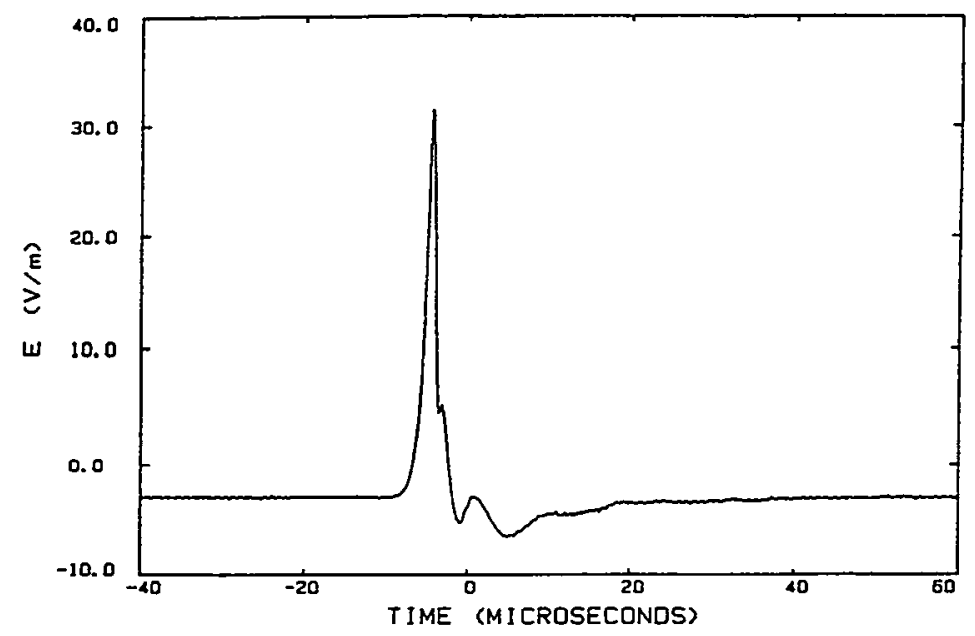

203928.628

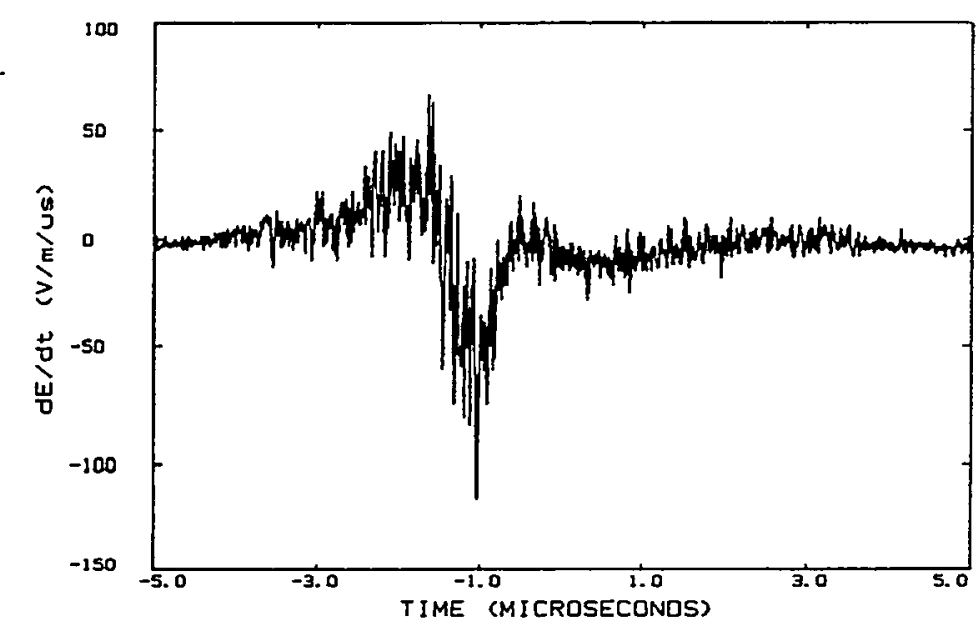

Fig. 2. Electric field $(E)$ and field-derivative ( $d E / d t$ ) waveforms for a typical narrow positive bipolar pulse (NPBP) from an isolated thunderstorm on July 24,1985 , centered $45 \mathrm{~km}$ away from our antennas. The "physics" sign convention is used here. Thus this pulse has the same polarity as those shown by Le Vine [1980], who used the opposite sign convention, and is opposite in polarity to a return stroke lowering negative charge (see Figure 3). Note the noisy appearance of $d E / d t$ (shown on an expanded time seale) in comparison with $E$.

Figure 1.10: A narrow positive bipolar electric field change pulse recorded by Willett et al. [1989]. From J. C. Willett, J. C. Bailey, and E. P. Krider, Journal of Geophysical Research, 94, p. 16257, 1989, copyright by the American Geophysical Union. 
few microseconds. Within a 30 event subset of the recorded bipolar pulses, two thirds were found to be isolated pulses that occurred with time separations of greater than one second from other lightning activity.

Electric field waveforms similar to those observed by Le Vine [1980], Willett et al. [1989], and Medelius et al. [1991] were described by Weidman and Krider [1979], Cooray and Lundquist [1985], and Bils et al. [1988]. The observations by these authors, however, were of pulses that lasted significantly longer and did not occur in isolation. The pulses were probably not from the same intracloud process that produces NPBPs.

\subsection{COMPACT INTRACLOUD DISCHARGES (CIDS)}

NPBPs, TIPPs, and SIPPs are produced by the same electrical discharges within thunderstorms. First evidence of this association was presented by Shao et al. [1996], Smith et al. [1996], and Holden et al. [1996]. Smith et al. [1997, b] presented calculations of the inferred physical properties of the discharge and provided evidence that the discharges were distinct from those that produce normal intracloud and cloudto-ground thunderstorm electrical emissions. These unique discharges and the source regions that produce them are the primary topics of this dissertation. As a convenience, the sources of the distinct electrical emissions shall be referred to as CIDs (compact intracloud discharges) throughout the remainder of this paper. 
Chapter 2 describes the instrumentation used to make measurements of thunderstorm electrical processes during the summer of 1996 . The instrumentation includes the three-station electric field change array, the three-station broadband HF array, and the Blackbeard broadband satellite receiver. Chapter 3 provides a description of the techniques used to locate emission sources using methods of time of arrival. Reflections of signals from the ionosphere and earth provided a powerful means of accurately determining source altitudes. Chapter 4 describes observations of three nighttime convective airmass thunderstorms that produced CIDs. The storms occurred in the southwestern US during July and August of 1996. Chapter 5 describes observations of tropical cyclone Fausto, which produced CIDs on two different nights in September of 1996. Chapter 6 summarizes observations of the recorded emissions in detail and provides an overview of their source regions. In Chapter 7, the phenomenology of CID emissions is used to determine fundamental characteristics of the source region and the discharge itself. Chapter 8 provides a final summary of the conclusions. For the reader's convenience, a glossary of terms and acronyms has been provided following Chapter 8 . 


\section{CHAPTER 2}

\section{INSTRUMENTATION}

Two arrays of ground-based sensors in northern New Mexico were utilized to observe and locate radio emissions from thunderstorms during the summer of 1996. Data and timing information from the Blackbeard instrument on the ALEXIS satellite were also used to locate and characterize thunderstorm emissions.

The first array consisted of three independent electric field change meters equipped with digital data acquisition and GPS (Global Positioning System) timing systems. The stations were separated by distances ranging from 30 to $230 \mathrm{~km}$. The locations of emission sources were determined from differential times of arrival (DTOAs) of signals recorded by the stations. Reflections of field change signals from the ionosphere and ground were often recorded by the stations in addition to the groundwave signals. The reflections provided vertical time of arrival (TOA) baselines for the determination of accurate three-dimensional (3-D) source locations.

The second array consisted of three broadband HF data acquisition systems also equipped with digital data acquisition and GPS timing. The stations were separated by distances ranging from 6 to $13 \mathrm{~km}$. Unlike the field change systems, the $\mathrm{HF}$ systems were not triggered independently. A VHF communication system transmitted trigger signals from the primary station to the two remote stations each time the 
primary station received a trigger. The communication system ensured that the three stations acquired data approximately coincidentally. HF times of arrival were also used in the determination of 3-D source locations.

Figure 2.1 shows the locations of the three fast electric field change systems (FC1, FC2, and FC3) and three broadband HF systems (HF1, HF2, and HF3). The primary HF system (HF1) was co-located with the Los Alamos electric field change system (FC1) at LANL. The Albuquerque NEXRAD weather surveillance radar $(\mathrm{ABX})$, from which radar reflectivity data were acquired, is also shown, along with the locations of three thunderstorms $(1,2$, and 3$)$ from which the ground-based observations described in Chapter 4 were made.

\subsection{ELECTRIC FIELD CHANGE INSTRUMENTATION}

The locations of the three electric field change systems (FC1, FC2, and FC3) are represented by circles in Figure 2.1. FC1 was operated by LANL from Los Alamos, NM. The field change meter was co-located with the primary broadband HF station, which is described in the following section. FC2 was operated by the New Mexico Institute of Mining and Technology (NMIMT) from the Langmuir Laboratory for Atmospheric Research, located $27 \mathrm{~km}$ west of Socorro, NM. FC3 was also operated by NMIMT, but from a residence located $6 \mathrm{~km}$ north-northeast of Socorro. The distances between the stations were (FC1-FC2) $224 \mathrm{~km}$, (FC2-FC3) $30 \mathrm{~km}$, and (FC3FC1) $206 \mathrm{~km}$. 


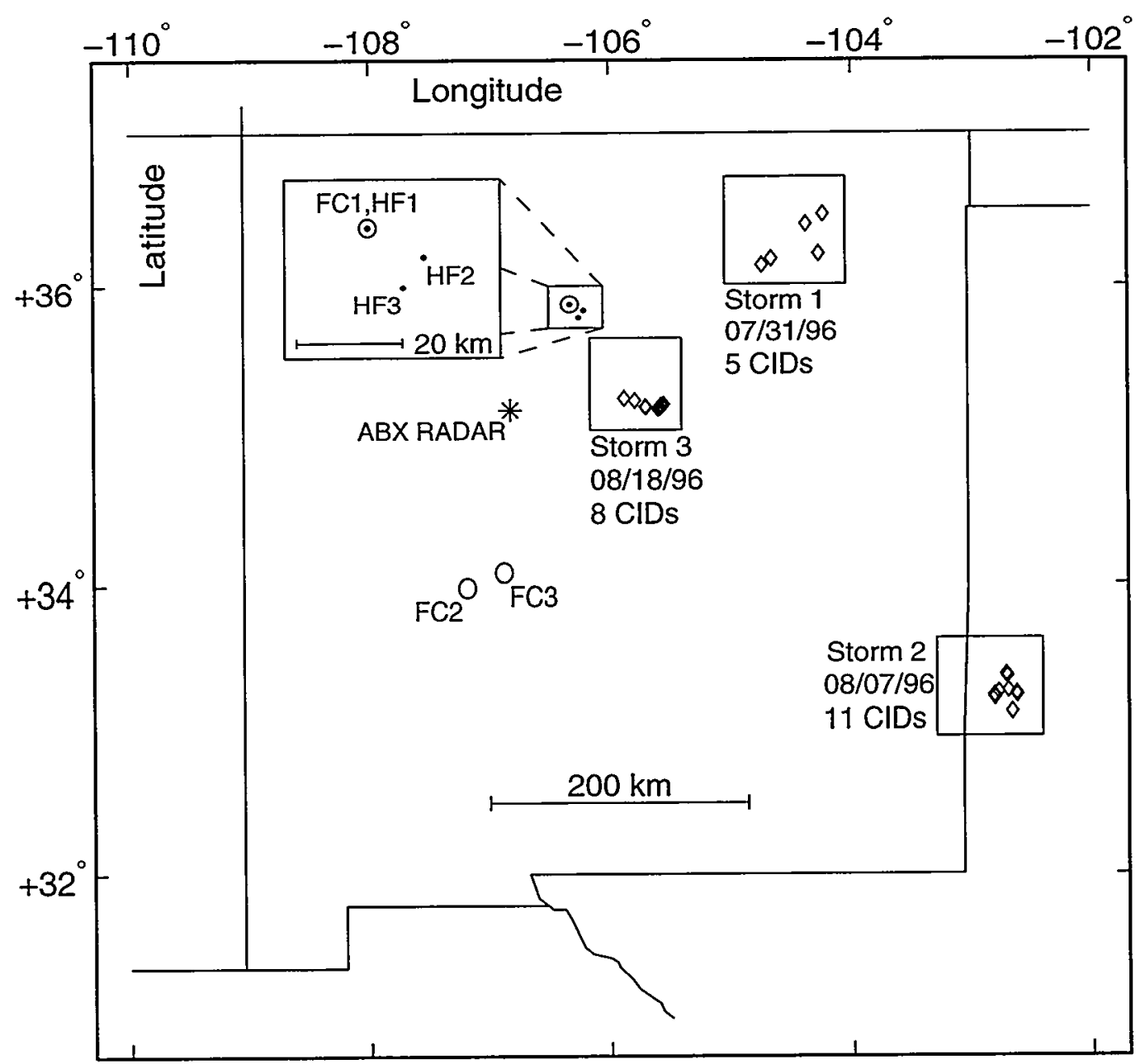

Figure 2.1: Plan view locations of the electric field change stations (FC1, FC2, and FC3), the broadband HF stations (HF1, HF2, and HF3), and the Albuquerque NEXRAD radar (ABX). Also shown are the locations of the three thunderstorms $(1,2$, and 3$)$ that were studied during the summer of 1996 . CID locations in the storms are represented by diamonds. 


\subsubsection{Field Change Meters}

The electric field change meters were similar in principle to the fast antenna system described by Kitagawa and Brook [1960] and nearly identical to the field change sensors described by Krehbiel et al. [1979] . The meters measure VLF/LF changes in the vertical component of the electric field at the ground. A differential measurement is made because the electric field change meters permit greater sensitivity to events on microsecond and millisecond time scales than sensors of absolute electric field. This greater sensitivity is achieved by allowing the output of the meter to decay to zero in between the individual events that occur during a single lightning flash.

In this paper the "physics" sign convention for electric field change polarity is used. Under this convention, the transfer of negative charge from ground to overhead produces a positive-polarity electric field change as measured from the ground. Note that the transfer of positive charge from overhead to ground would also produce a positive-polarity electric field change signal. A negative cloud-to-ground return stroke, the type of ground stroke that occurs most commonly in most thunderstorms, lowers negative charge and thus produces an initially-negative-polarity field change signal.

A photograph of the author standing next to a LANL electric field change meter is shown in Figure 2.2. The sensing portion of the meter and the electronics associated with charge amplifier and line driver circuitry are located underneath the $45 \mathrm{~cm}$ diameter grounded dome. The dome provides electromagnetic shielding for 


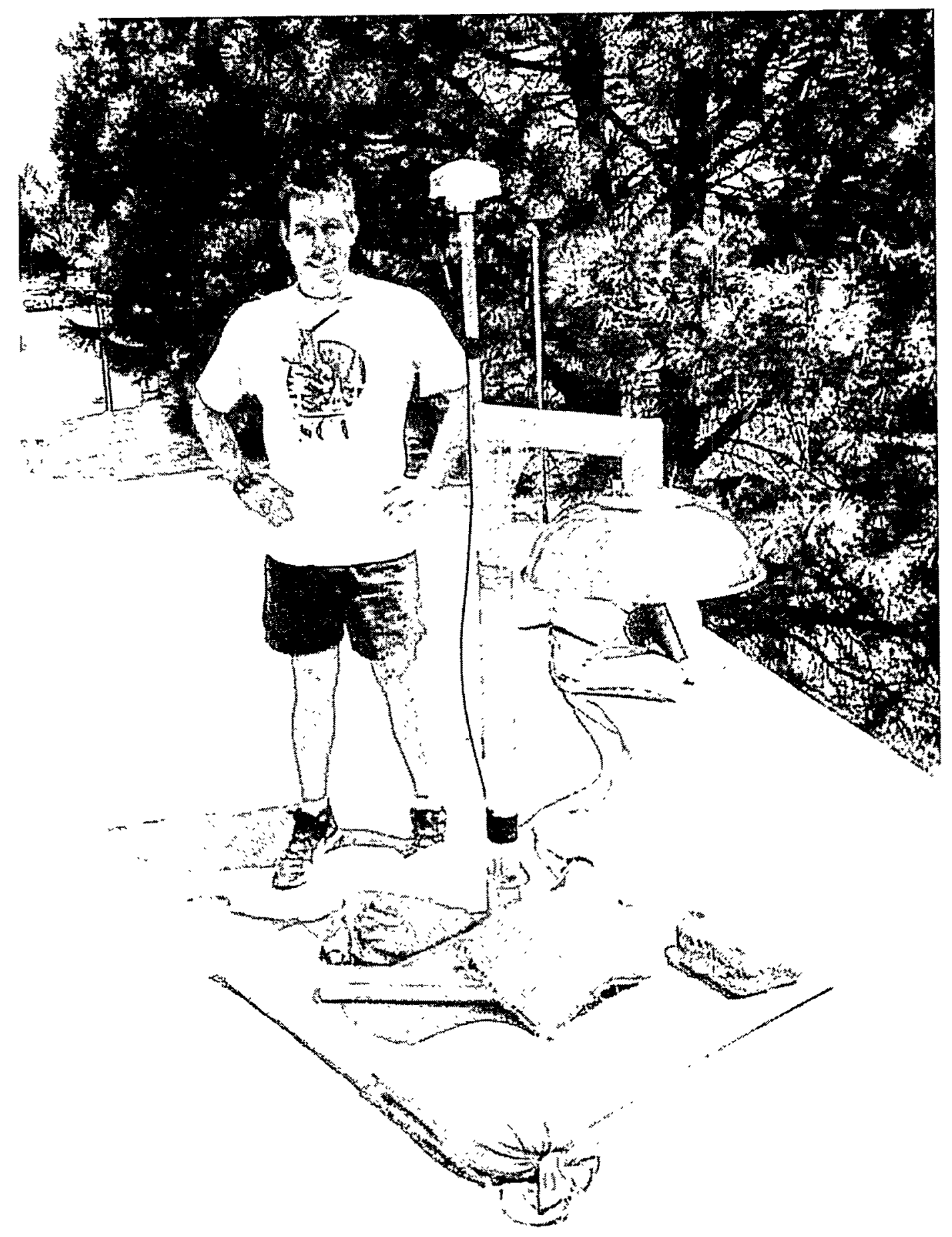

Figure 2.2: A photograph of the author and an electric field change meter at Los Alamos National Laboratory. The sensing plate of the field change meter sits underneath the inverted "salad bowl." A GPS antenna protrudes from the field change meter stand. 
the electronics and prevents rain from contacting the sensing plate of the electric field change meter. A GPS antenna/receiver is also shown in the photo. It sits atop the pole that protrudes from the field change meter stand.

A schematic diagram of the electric field change meter sensing circuit is shown in Figure 2.3. The sensing plate is a $44 \mathrm{~cm}$ diameter $1 / 8$ inch thick aluminum disk. It provides input to an op-amp circuit configured as a charge amplifier. The system is sensitive to changes in the electric field at the sensing plate. When an electric field change $(\Delta E)$ occurs, charge is induced on the plate $(\Delta Q)$. The amount of charge is given by Equation 2.1:

$$
\Delta Q=A_{e f f} \varepsilon_{o} \Delta E
$$

where $A_{\text {eff }}$ is the effective area of the plate and $\varepsilon_{o}$ is the permittivity of free space. The voltage change $(\Delta V)$ at the output terminals of the circuit for a large resistor value $(R)$ is given by Equation 2.2:

$$
\Delta V=\frac{\Delta Q}{C}=\frac{A_{e f f} \varepsilon_{o}}{C} \Delta E
$$

where $C$ is the capacitance of the capacitor in the circuit. Equation 2.2 shows that the voltage change at the output of the circuit is directly proportional to the ambient electric field change. The resistor in the circuit provides a discharge path for the charge induced on the capacitor following an electrostatic field change. Thus, after a 


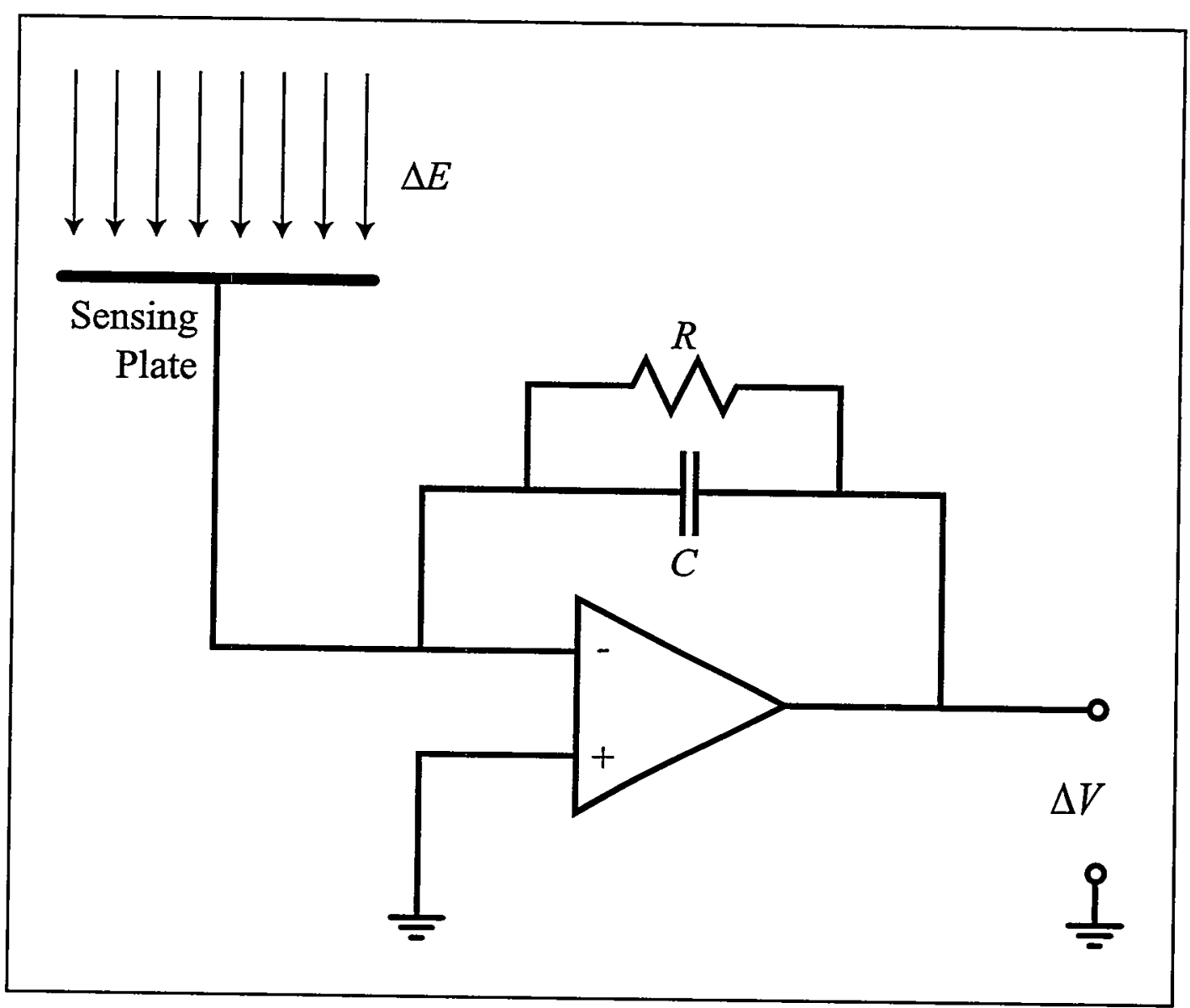

Figure 2.3: A schematic diagram of an electric field change meter charge amplification circuit. 
shift in the ambient field (like that produced by a close lightning discharge), the output voltage step from the field change meter will decay to zero with a time constant of $R C$.

The field change meters used during this study have often been referred to as "fast" antenna systems because their $R C$ time constants are relatively short compared to lightning flashes. The time constants are between several hundred microseconds and several milliseconds. Thus the meters are sensitive to intra-flash thunderstorm phenomena, such as multiple return strokes and K-changes. "Slow" antennas are also powerful tools for thunderstorm research. Such systems have time constants of many seconds and are sensitive to gross charge motion integrated over the duration of an entire flash. The slow systems provide a largely electrostatic picture of charge redistributions associated with thunderstorm electrical discharges.

Figure 2.4 shows a block diagram of the LANL electric field change system (FC1). A line driver in the field change meter was used to feed a $50 \Omega$ coaxial line that passed indoors to a power splitter. The splitter provided input to a digital delay generator and a digital oscilloscope. The delay generator was used to determine the trigger threshold of the system and to provide simultaneous trigger signals to the oscilloscope, the time tag buffer of the GPS receiver, and the LANL HF1 data acquisition system. When a trigger occurred, the oscilloscope acquired the electric field change waveform and the GPS receiver recorded a UTC (Coordinated Universal Time) time tag. Time tags permitted the estimation of source locations based on differential times of arrival at multiple stations. Following the acquisition of each waveform with its associated time tag, the data were transferred to a computer and 


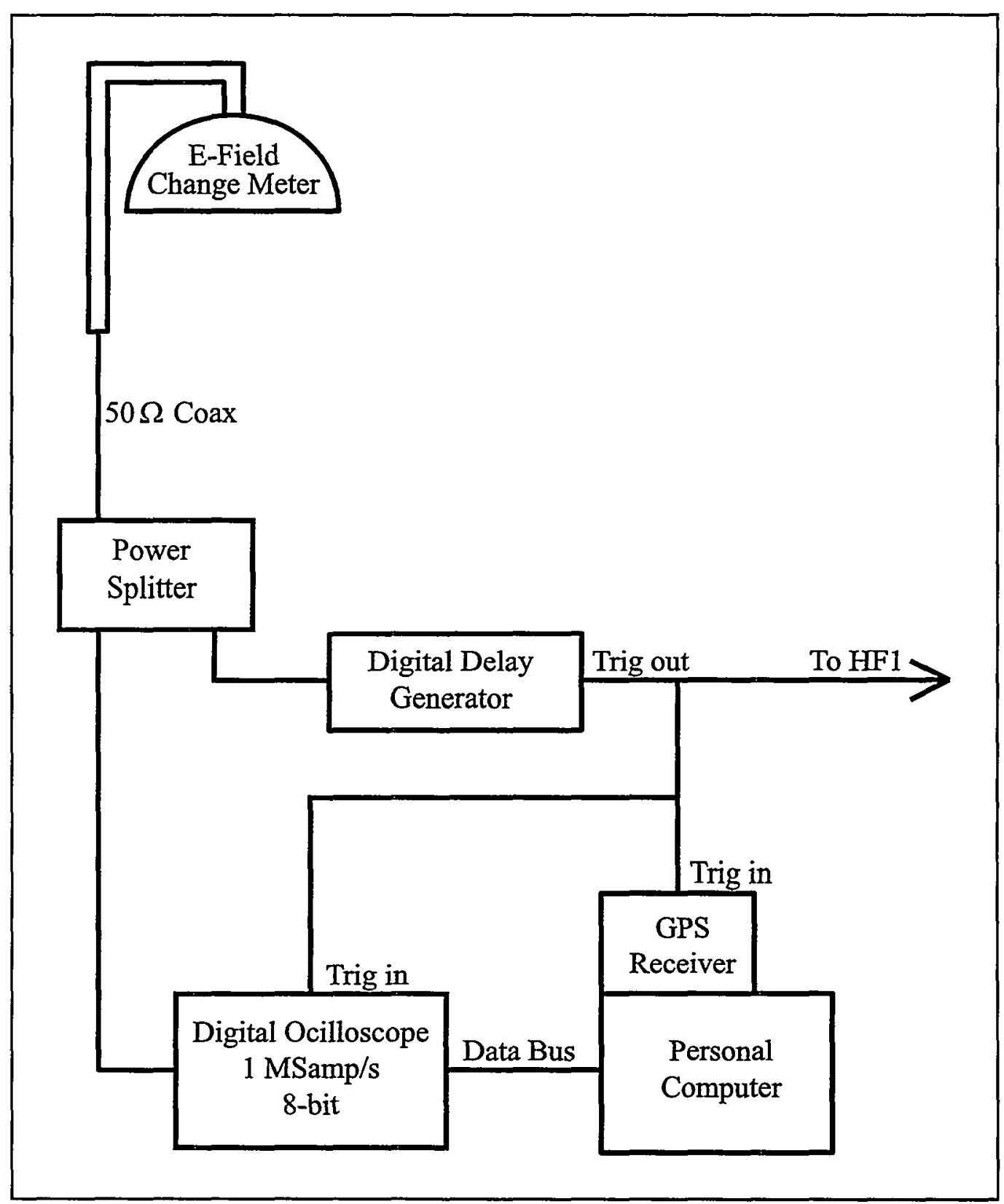

Figure 2.4: Block diagram of the LANL electric field change data acquisition system (FC1). A trigger signal from the field change meter was used to trigger the LANL broadband HF systems. 
saved for later analysis. The FC2 and FC3 systems were similar in most respects to the LANL system.

Each of the three field change meter data acquisition systems was triggered from a positive-slope threshold on the field change meter output. Digitizers were configured to record data with a predetermined pretrigger fraction (always $1 / 4$ to $1 / 2$ ) in order to acquire field change data both before and after triggering events. The absolute timing uncertainty for each of the three field change stations was less than 2 $\mu$ s. Operational characteristics of the three electric field change data acquisition systems are summarized in Table 2.1 .

\begin{tabular}{|l|c|c|c|}
\hline & Los Alamos (FC1) & Langmuir (FC2) & Socorro (FC3) \\
\hline Rise Time & $<1 \mu \mathrm{s}$ & $<1 \mu \mathrm{s}$ & $<1 \mu \mathrm{s}$ \\
Decay Time Constant $(R C)$ & $100 \mu \mathrm{s}$ & $1 \mathrm{~ms}$ & $6 \mathrm{~ms}$ \\
Digitizer Bits & 8 & 8 & 12 \\
Sampling Rate & $1 \mathrm{Msamp} / \mathrm{s}$ & $0.5 \mathrm{Msamp} / \mathrm{s}$ & $2 \mathrm{Msamp} / \mathrm{s}$ \\
Typical Record Lengths & 5 or $50 \mathrm{~ms}$ & $\sim 4 \mathrm{~ms}$ & $\sim 8 \mathrm{~ms}$ \\
Absolute Timing Uncertainty & $1 \mu \mathrm{s}$ & $2 \mu \mathrm{s}$ & $1 \mu \mathrm{s}$ \\
\hline
\end{tabular}

Table 2.1: Summary of the operational characteristics of the three electric field change data acquisition systems. 


\subsubsection{Field Change Meter Calibration}

It was necessary to calibrate the outputs of the three field change meters in order to make quantitative statements about electric field change amplitudes recorded from thunderstorm discharges. To perform a system calibration at a given "permanent" field change meter site, a second, "reference" field change meter was temporarily set up at the same location. The calibration procedure required two steps. First, the reference meter was calibrated by setting up a well-characterized alternating electric field in the vicinity of its sensing plate. Second, the reference and permanent meters were used to simultaneously observe actual electric field change events. The ratio of the outputs of the meters was used to determine the calibration factor for the permanent meter.

Figure 2.5 shows a diagram of the reference electric field change meter that was used to calibrate the three field change systems. The reference meter consisted of a flat plate antenna ( $30 \mathrm{~cm}$ diameter) that was mounted flush within a hole in a large circular aluminum disk (100 $\mathrm{cm}$ diameter). The outer disk was connected to ground potential. The role of the disk was to reduce fringe effects at the edge of the flat plate antenna and to assure that the plane of the antenna was at ground potential. A second aluminum disk of the same diameter as the first, but without a hole, was placed above the reference antenna at a known standoff $(9 \mathrm{~cm}$ for the LANL calibration) and was fed by a low-frequency (compared to the time constant of the field change meter) square wave signal with a known amplitude ( $5 \mathrm{~V}$ peak-to-peak for the LANL calibration). Since the spacing between the plates $(s)$ was known and the time- 


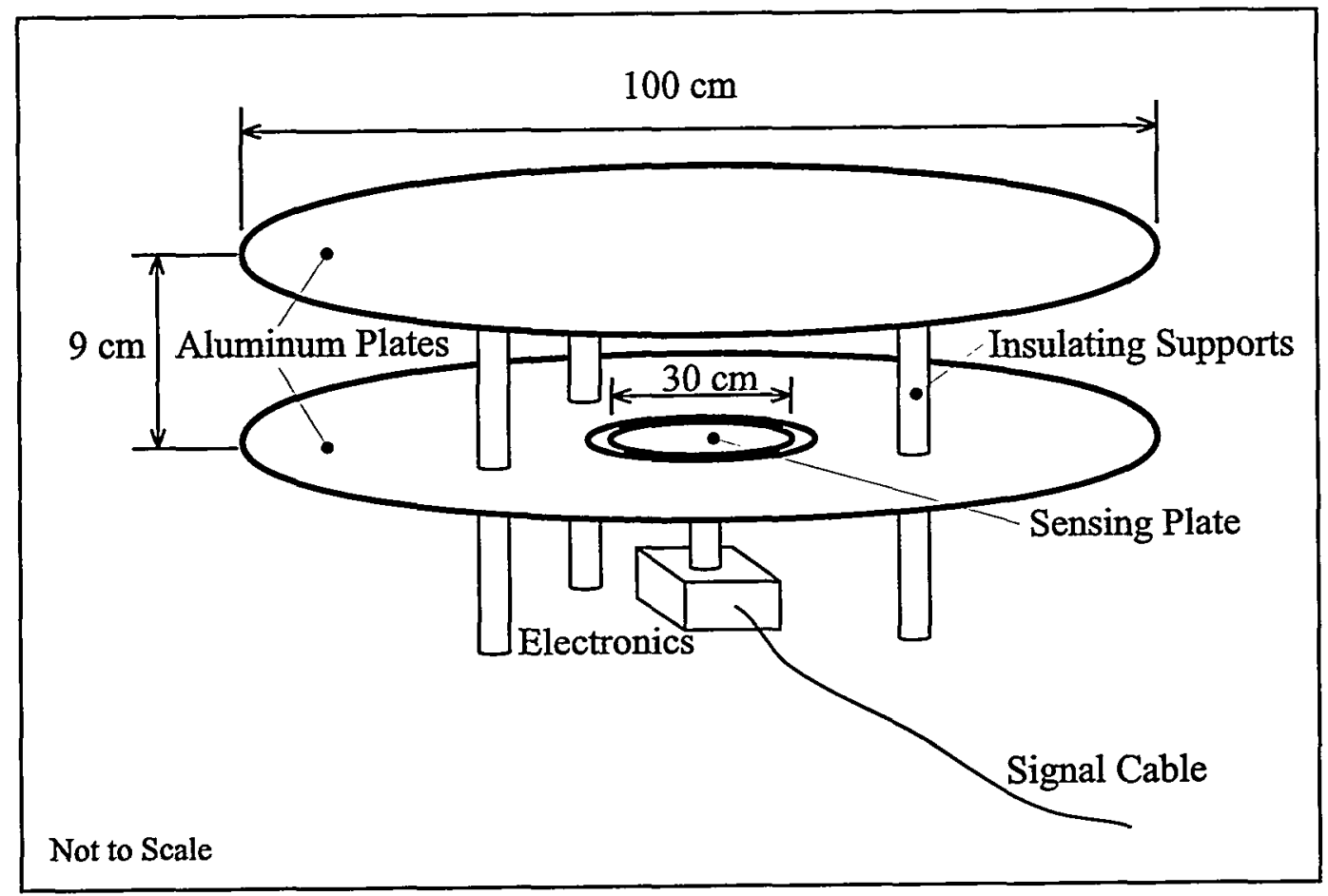

Figure 2.5: Illustration of the calibration electric field change meter. The lower $100 \mathrm{~cm}$ aluminum plate was connected to ground potential. During calibration, a square wave signal was connected to the upper plate to determine the response of the meter to a known oscillatory electric field. 
varying input voltage $(V)$ was known, the time varying electric field between the large disks $(E)$ was known and given by Equation 2.3:

$$
E=\frac{V}{s}
$$

For the LANL calibration this field was approximately $56 \mathrm{~V} / \mathrm{m}$. In Equation 2.3 it is assumed that fringe effects at the edge of the flat plate antenna are negligible, so that the field is known and uniform.

The voltage output of the reference field change meter was observed and recorded as a function of the input electric field. The voltage change corresponding to the known electric field change was noted. For the LANL electric field change meter, it was observed that a $1.00 \mathrm{~V}$ change in the reference meter output was produced by an electric field change of $21.7 \mathrm{~V} / \mathrm{m}$.

Having calibrated the reference field change meter, the upper calibration plate was removed and the meter was used to observe spherics (short for "atmospherics", a general term used to describe transient, low frequency radio signals from thunderstorm discharges) in conjunction with the permanent field change meter at the site. Amplitudes of spherics recorded by both meters were compared to each other in order to determine the relative calibration factor between the two systems. The average ratio between the peak amplitudes of events recorded by the LANL station and those recorded by the reference station was 0.625 , so it was determined that a $1.00 \mathrm{~V}$ change in the output of the LANL electric field change meter corresponded to 
a $34.8 \mathrm{~V} / \mathrm{m}$ change in the local electric field. This procedure was used to calibrate each of the three electric field change systems.

It may be questioned why the reference field change meter was necessary to perform the calibration: Why not simply set up a known alternating field and measure it with the permanent meter? The answer to this question is that for practical purposes it is difficult to set up a well-characterized calibration field in the vicinity of the permanent meters because their physical configurations are complex compared to that of the reference meter. Their flat plate antennas are housed beneath grounded umbrellas that enclose the electronics and prevent rain from contacting the sensing plate. Shielding from rain is necessary because contact of the sensing plate by charged raindrops can produce undesirable voltage transients at the input (and thus output) of the field change meter.

\subsection{BROADBAND HF INSTRUMENTATION}

The locations of the three broadband HF systems (HF1, HF2, and HF3) are represented by dots in Figure 2.1. The primary broadband HF station (HF1) was colocated with the Los Alamos electric field change station (FC1) at LANL. A second HF station (HF2) was located in the community of White Rock, $12 \mathrm{~km}$ east-southeast of Los Alamos. A third station (HF3) was located at a remote LANL site $13 \mathrm{~km}$ south-southeast of Los Alamos. The distances between the stations were (HF1-HF2) $11.8 \mathrm{~km}$, (HF2-HF3) $6.9 \mathrm{~km}$, and (HF3-HF1) $12.8 \mathrm{~km}$. 


\subsubsection{Broadband HF Receivers}

The three broadband HF systems used to record thunderstorm emissions during the summer of 1996 were similar to the system described by Smith [1995] and Smith and Holden [1996], the primary difference being the triggering method. Operation in the HF portion of the radio spectrum meant that it was possible to receive ionospheric reflections of RF (radio frequency) signals under favorable ionospheric conditions. A block diagram of the HF1 system is shown in Figure 2.6.

Discone antennas at each of the three stations were used to receive $\mathrm{HF}$ radio emissions. The author is shown in Figure 2.7 standing next to the LANL HF1 discone. The $13 \mathrm{~m}$ tall antennas achieve maximum gain near the horizon and have an azimuthally symmetric radiation pattern. A smaller, $9 \mathrm{~m}$ discone was utilized at the HF2 station for reasons of practicality. The antennas are vertically polarized. General discone antenna design was discussed by Kandoian [1946]. A specific design similar to the one used for the present study was described by Belrose [1975]. Signals received by each of the antennas passed through $50 \Omega$ coaxial lines indoors where they were filtered by $3 \mathrm{MHz}$ highpass and $30 \mathrm{MHz}$ lowpass filters. The highpass filters had a cutoff slope of $40 \mathrm{~dB} / \mathrm{MHz}$ down to $-60 \mathrm{~dB}$. The lowpass filters had a cutoff slope of $10 \mathrm{~dB} / \mathrm{MHz}$ down to $-40 \mathrm{~dB}$. Signals were also passed through diode protection circuits before passing to the digital oscilloscopes. The diode circuits prevented equipment damage due to high voltages on the signal line resulting from local lightning discharges. Data were recorded by 8-bit digital oscilloscopes configured to sample at $50 \mathrm{Msamp} / \mathrm{s}$. Note that the Nyquist frequency for the 


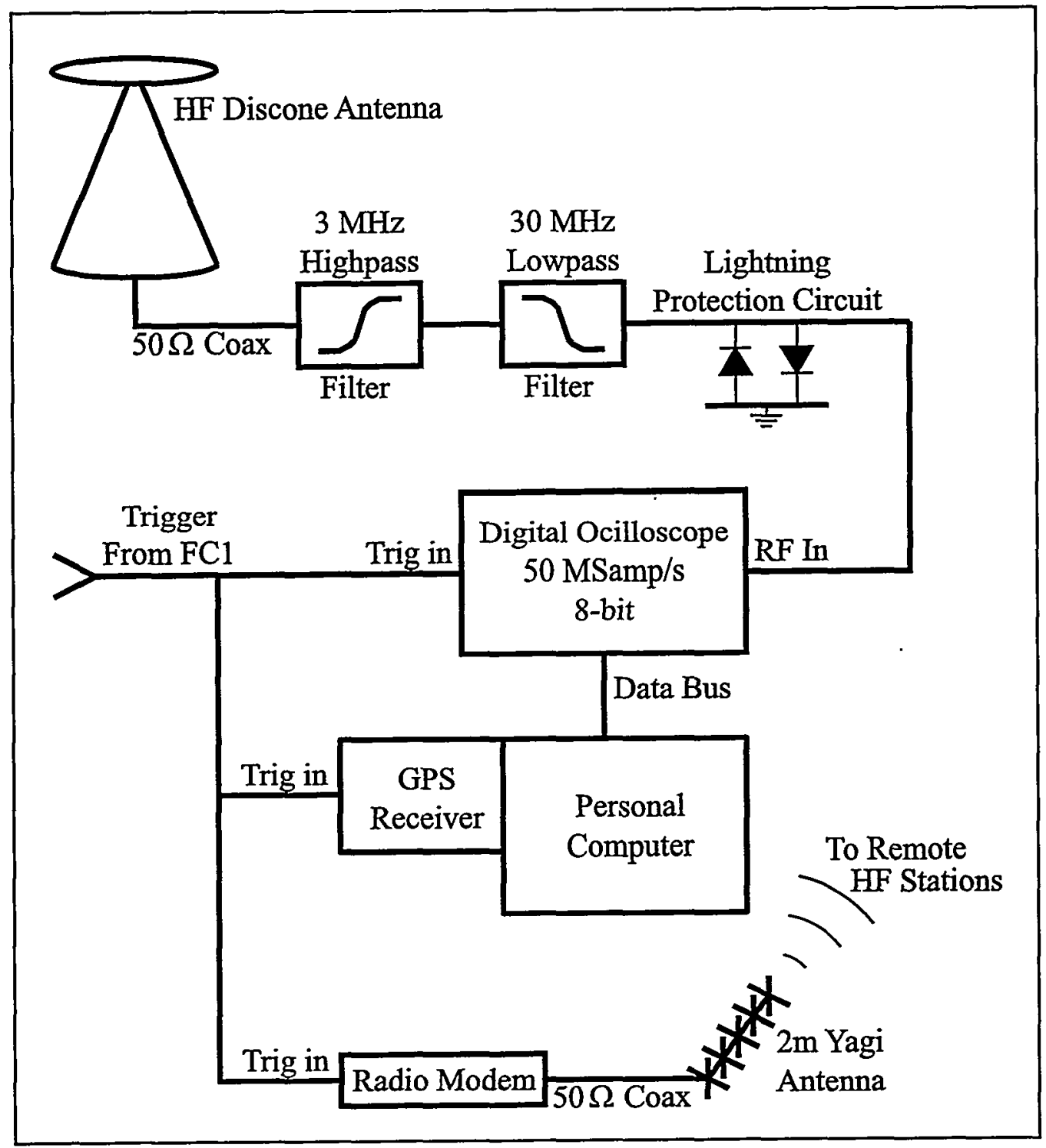

Figure 2.6: Block diagram of the LANL broadband HF data acquisition system (HF1). The system received its trigger from the LANL electric field change system (FC1), recorded broadband HF and timing data, and transmitted a trigger signal to the remote HF stations (HF2 and HF3) via a VHF communication link. 


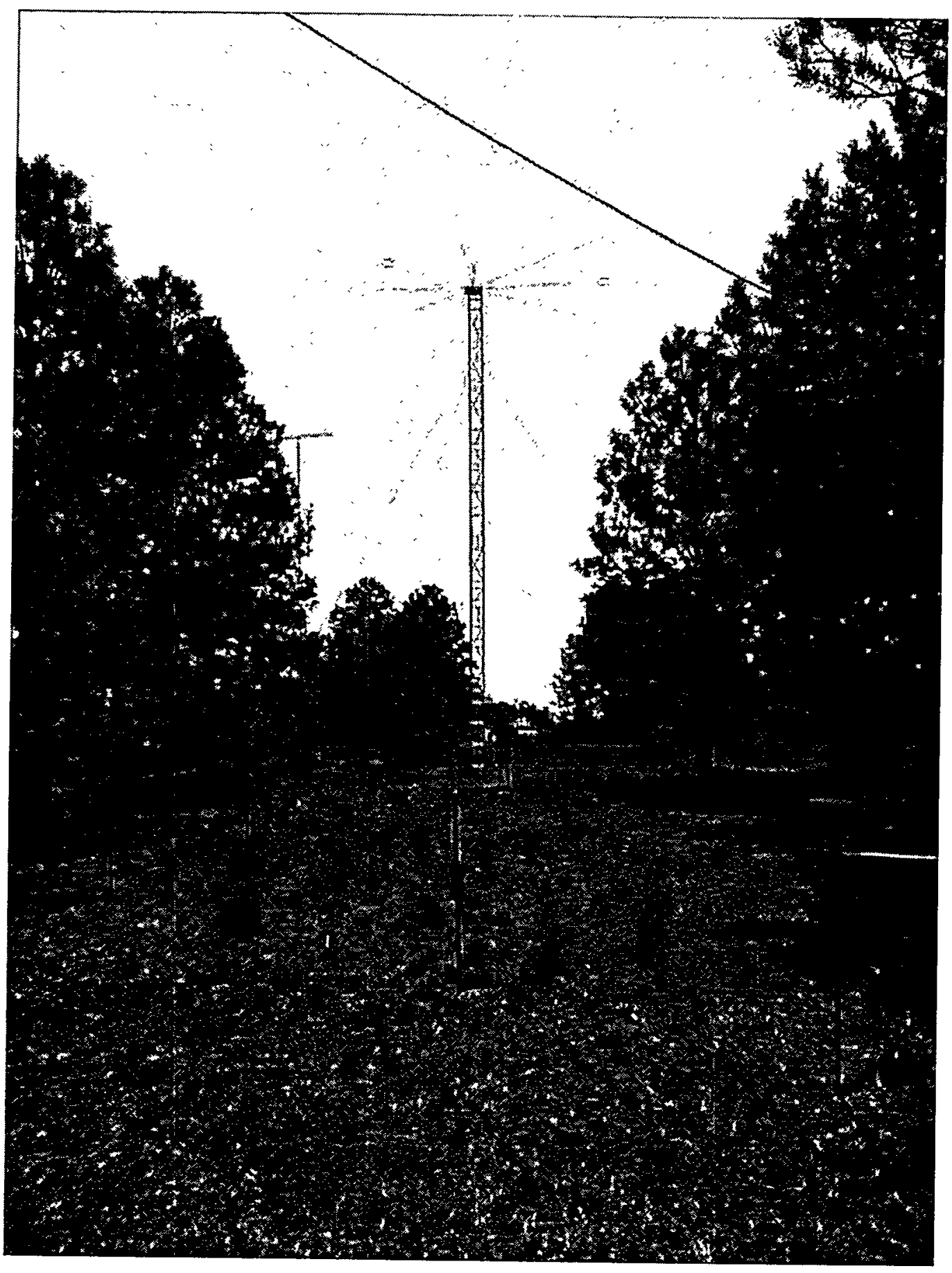

Figure 2.7: A photograph of the author and one of the LANL HF discone antennas (at the HF1 site). The antenna is $13 \mathrm{~m}$ tall. 
sampling rate was $25 \mathrm{MHz}, 5 \mathrm{MHz}$ below the $30 \mathrm{MHz}$ input lowpass frequency. Thus input frequencies between 25 and $30 \mathrm{MHz}$ were aliased to the 20 to $25 \mathrm{MHz}$ range, which was compensated for through the use of digital signal processing. For all data presented in this dissertation, $1 \mathrm{~ms}$ (50 kpoint) records were acquired.

HF1 received its trigger signal from the LANL electric field change data acquisition system (FC1). Each time FC1 triggered on a field change waveform, it sent trigger signals to the HF1 digital oscilloscope, the HF1 GPS time tag buffer, and a VHF radio modem. Thus HF waveforms and time tags were recorded by HF1, but also trigger signals were transmitted to each of the remote HF stations via a communication link. VHF radio modems using an FSK (frequency shift keying) modulation scheme were utilized to achieve the "trigger transfer." The remote stations were configured with additional pretrigger to allow for the propagation delays from the primary station and the delays that characterized the communication link (together approximately $600 \mu \mathrm{s}$ ). Effectively, the trigger transfer system allowed all three HF stations to be triggered from the LANL field change acquisition station (FC1) simultaneously. The typical pretrigger fraction was $3 / 10$ for each of the HF stations.

Like events recorded by the field change meters, events recorded by each of the three HF stations were time stamped using GPS receivers. The absolute timing uncertainty for the HF time tags was less than $2 \mu \mathrm{s}$. 


\subsubsection{Broadband HF System Calibration}

The frequency response of the LANL HF system (HF1) was determined using a combination of transmission and reflection measurements and modeling. For convenience, it was assumed that the other two HF stations had similar frequency responses. The assumption was considered to be reasonable because the three stations utilized identical equipment with only two exceptions. The first exception was that a 2/3 scale, $9 \mathrm{~m}$ discone antenna was utilized at the HF2 station. A portable VSWR (voltage standing wave ratio) meter was used to determine the effects of using a smaller antenna. As expected, the response was only affected at low frequencies. Its smaller size caused the VSWR $=2$ cutoff to shift to $8 \mathrm{MHz}$ as compared to $6 \mathrm{MHz}$ for the two larger antennas. This fact was taken into consideration when the electric field amplitude calibrations were performed. The second exception was the fact that the coaxial feed lines at the three stations were of somewhat different types and lengths. Because the feed lines were all low loss coaxial cable $(<2 \mathrm{~dB} / 100 \mathrm{~m}$ at $\mathrm{HF})$ and only $\mathrm{HF}$ frequencies were of concern, the differences in loss were certainly less than $1 \mathrm{~dB}$.

The discone antenna was modeled using the NEC (Numerical Electromagnetics Code) antenna analysis software package. The program uses numerical methods to solve integral equations for the currents induced on the antenna structure by sources or incident fields. Using the NEC interface, a wire model of the antenna was constructed. Figure 2.8 shows the wire model of the discone antenna. The graphical depiction makes the antenna geometry more clear than the photograph of Figure 2.7. 


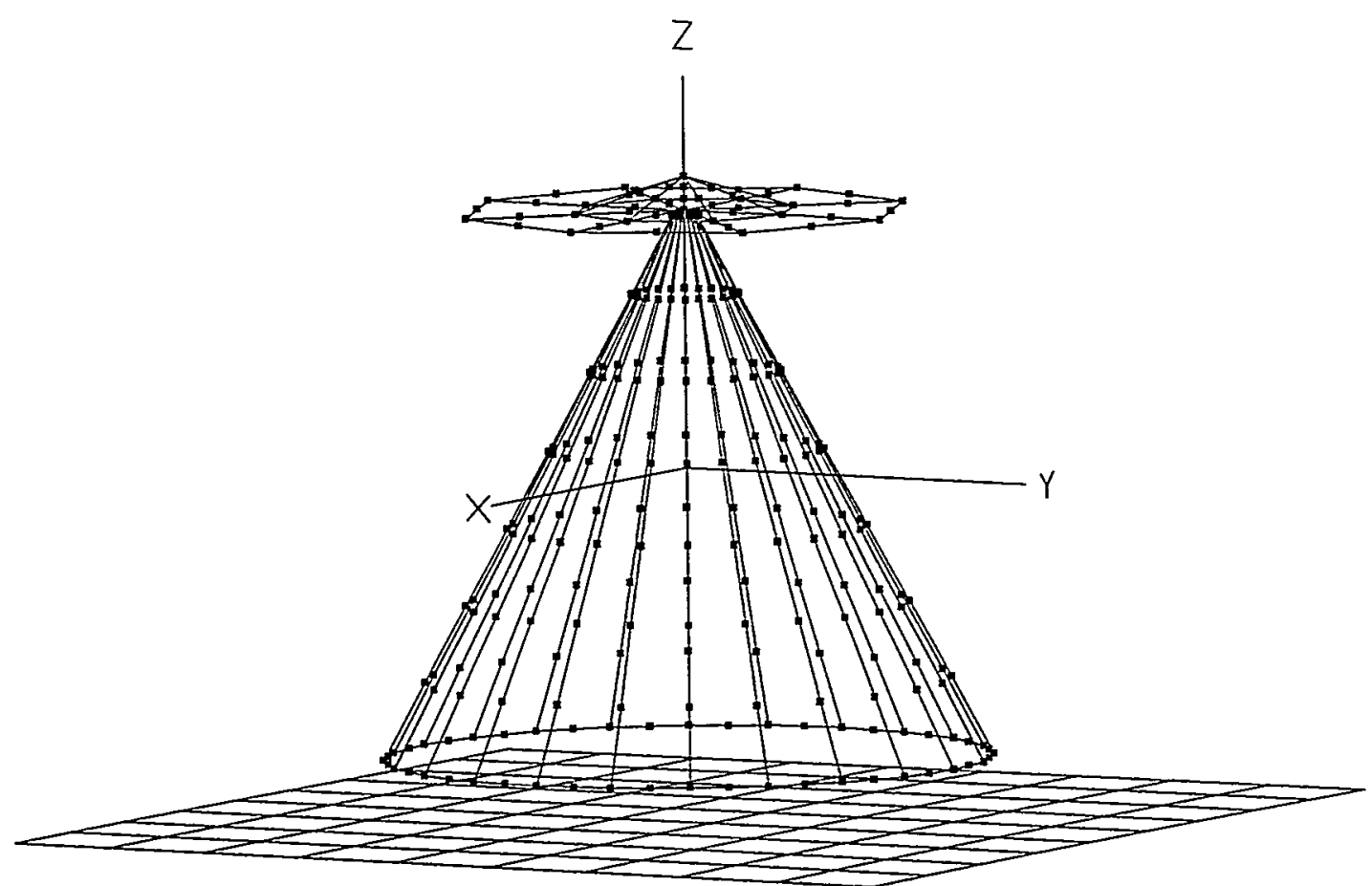

Figure 2.8: A computer-generated wire-frame model of an HF discone antenna. 
The software model was used to estimate the radiation pattern of the discone. The size $(13 \mathrm{~m})$ of the antenna would have made it difficult to perform far-field pattern measurements, especially at high elevation angles. Because the antenna is azimuthally symmetric, only the antenna response as a function of elevation angle was a required output of the NEC software.

Figure 2.9 shows the calculated discone antenna pattern from NEC as a function of elevation angle at six $\mathrm{HF}$ frequencies: $3,5,8,13,20$, and $30 \mathrm{MHz}$. A deep overhead null was clear at all frequencies tested. At lower frequencies (below around $12 \mathrm{MHz}$ ), a single, fat antenna lobe was predicted to exist near the horizon. At higher frequencies, multiple lobes were formed between the horizon and overhead null. The discone antenna was designed for maximum directivity at angles close to the horizon. For this reason it is useful for receiving obliquely propagated signals from the ionosphere that arrive from low elevation angles. NEC calculations of discone directivity ranged from -1 to $3 \mathrm{~dB}$ for all $\mathrm{HF}$ frequencies. The elevation angle of maximum directivity varied with frequency. Specific gain values were used with their corresponding elevation angles to compute normalized electric field strengths.

A network analyzer was used to measure the impedance of the antenna as a function of frequency. A plot of the actual VSWR of the discone as a function of frequency is shown as a solid line in Figure 2.10. The plot indicates that the antenna presents a very good match at frequencies between 6 and $20 \mathrm{MHz}$. Above $20 \mathrm{MHz}$ a mismatch exists; it is centered at a frequency of $25 \mathrm{MHz}$. The reason for the mismatch is not known, but it is consistent with the report of Belrose [1975], who described a mismatch centered at a frequency of $24 \mathrm{MHz}$ for a similar discone 

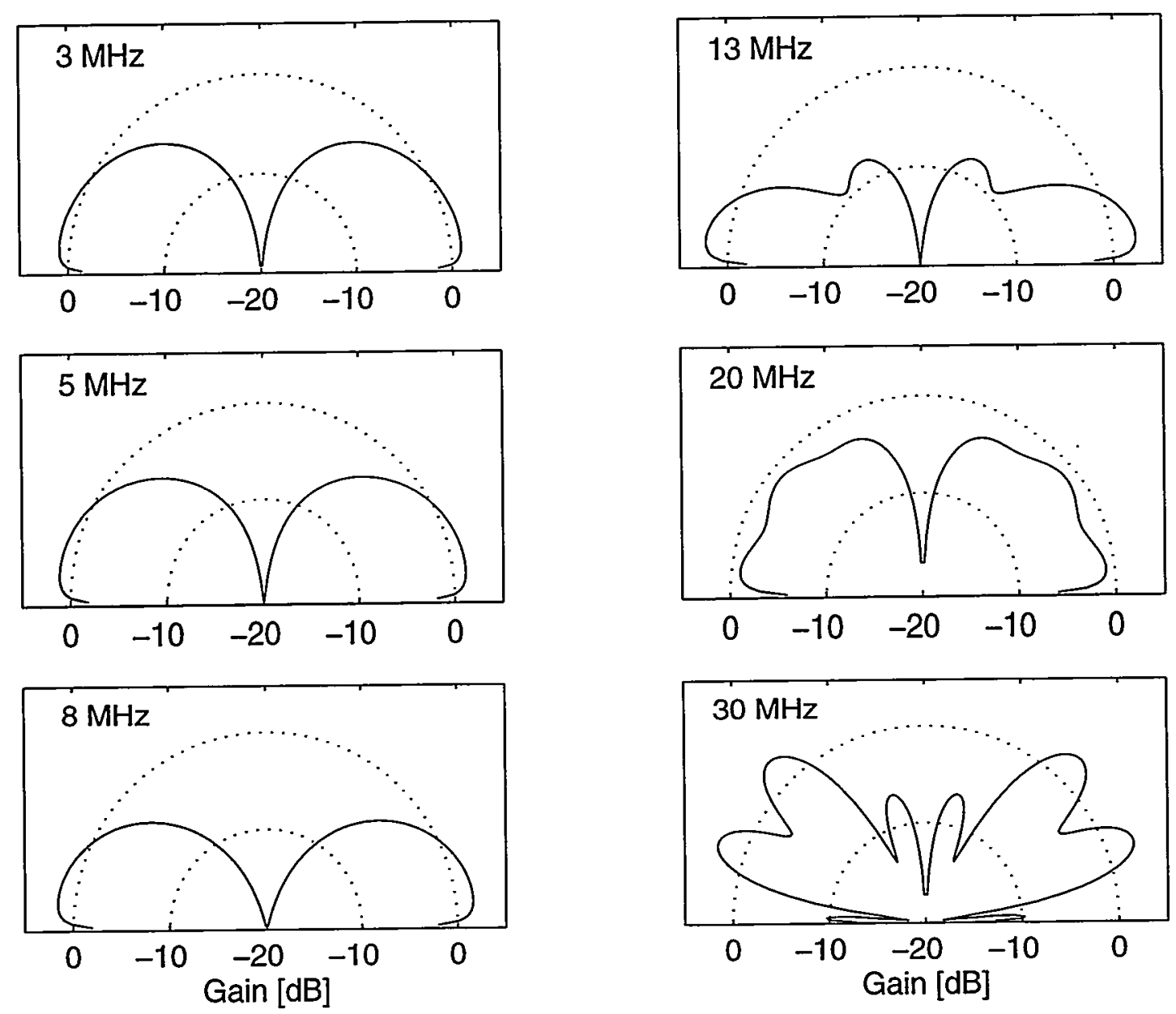

Figure 2.9: The modeled radiation pattern of the HF discone antenna as a function of elevation angle and frequency. 


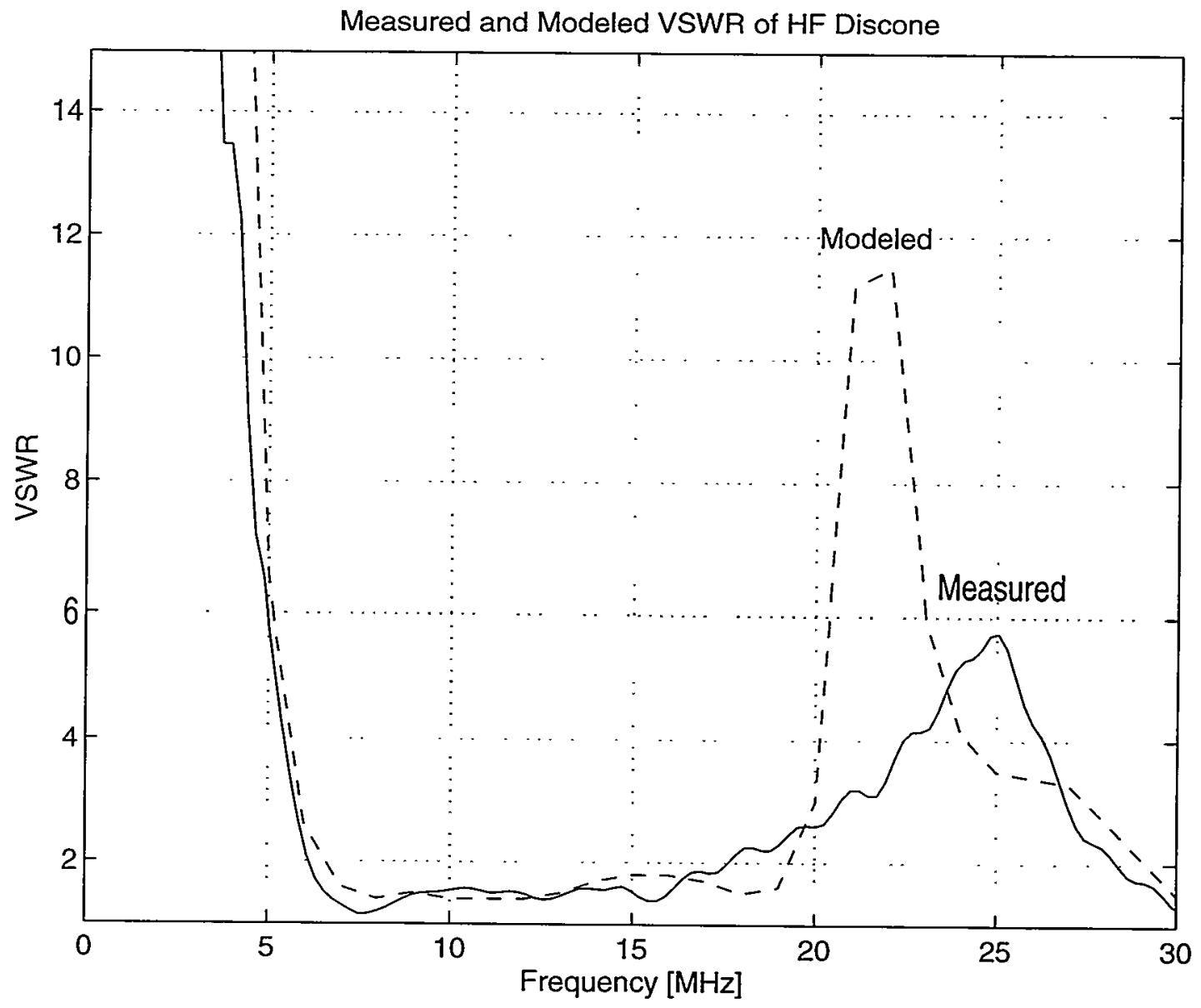

Figure 2.10: Comparison of the measured VSWR (voltage standing wave ratio) of the HF1 discone as a function of frequency to that of the modeled discone. 
antenna design. He claimed that it was probably due to nearby structures, but the consistent observations presented here provide evidence that it is an inherent characteristic of the design. The VSWR of the antenna as forecast by NEC is shown as a dashed line in Figure 2.10. The measured and modeled responses match well below $20 \mathrm{MHz}$. The modeled predicted the antenna mismatch, but at a lower frequency of $22 \mathrm{MHz}$. The fact that the mismatch was predicted by the modeling software further suggests that the mismatch is due to an inherent design characteristic.

In addition to the receiving antenna, all other components of the HF1 receiving system were characterized. The antenna was fed by approximately $60 \mathrm{~m}$ of coaxial cable: a combination of double-shielded RG-214 and FSJ4-50B 1/2" foamflex heliax. Between the transmission line and the digital recording equipment were two sharpcutoff filters, a $3 \mathrm{MHz}$ highpass and a $30 \mathrm{MHz}$ lowpass, and an over-voltage protection circuit. The protection circuit was simple in construction with two oppositely-biased diodes placed in parallel between the center conductor and outer conductor of the coaxial feed. Although the diodes did not have high current sinking capacity to protect the equipment against a direct strike, they provided adequate protection from high voltage transients caused by local lightning discharges. Figure 2.11 shows the one-way loss for the transmission line, filters, and protection circuit as a function of frequency for HF1.

Calibration of the antenna and signal path of the data acquisition system made it possible to determine actual vertical electric fields from the digital oscilloscope amplitude waveforms. In doing the conversions, simplifications were utilized. For example, in converting raw broadband waveforms to electric field, single 


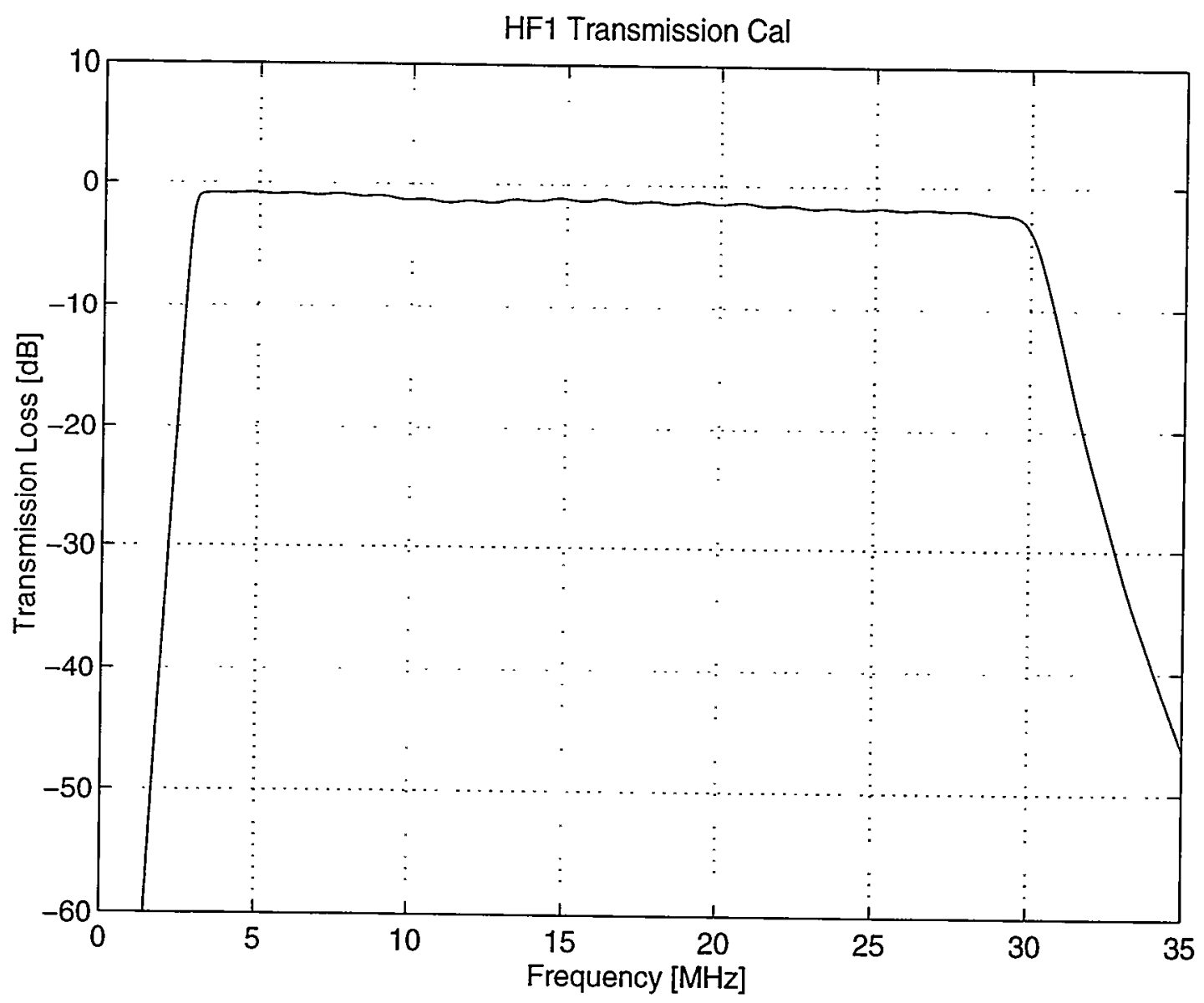

Figure 2.11: The transmission loss between the antenna and recording equipment at the LANL broadband HF station as a function of frequency. Included in the measurement were $\sim 70 \mathrm{~m}$ of coaxial cable, a $3 \mathrm{MHz}$ highpass filter, a $30 \mathrm{MHz}$ lowpass filter, and a circuit for shunting high transient voltages. 
representative values were used for the frequency dependent measurements (e.g. antenna impedance and transmission line loss). The simplification made it unnecessary to perform convolutions using the frequency dependent transfer functions. Discrepancies resulting from the simplification were minimized in the determination of normalized electric fields by using representative values at specific frequencies of interest.

\subsection{BLACKBEARD}

As described in Chapter 1, Blackbeard is a broadband radio science experiment on ALEXIS, a small satellite launched on 25 April 1993 into a $750 \times 850 \mathrm{~km}, 70^{\circ}$ inclination orbit. A photograph of ALEXIS is shown in Figure 2.12. A somewhat simplified Blackbeard block diagram is shown in Figure 2.13. Blackbeard takes its input from either of a pair of wideband sub-resonant (length $<\lambda / 4$ ) monopole antennas. The monopoles are backed by active preamplifiers within the antenna assemblies. The antennas cannot be seen in Figure 2.12, but extend from the top and the bottom of the spacecraft. In orbit, one antenna is always sunward-pointing. Input from an antenna switch between the two antennas passes through a gain stage, an attenuation stage, and a bank of selectable analog RF filters before being mixed to an intermediate frequency. The filters permit data acquisition within bandwidths smaller than $\sim 75 \mathrm{MHz}$ and have proven useful for rejecting noisy portions of the VHF spectrum. There are sixteen filter combinations in the Blackbeard low band and 


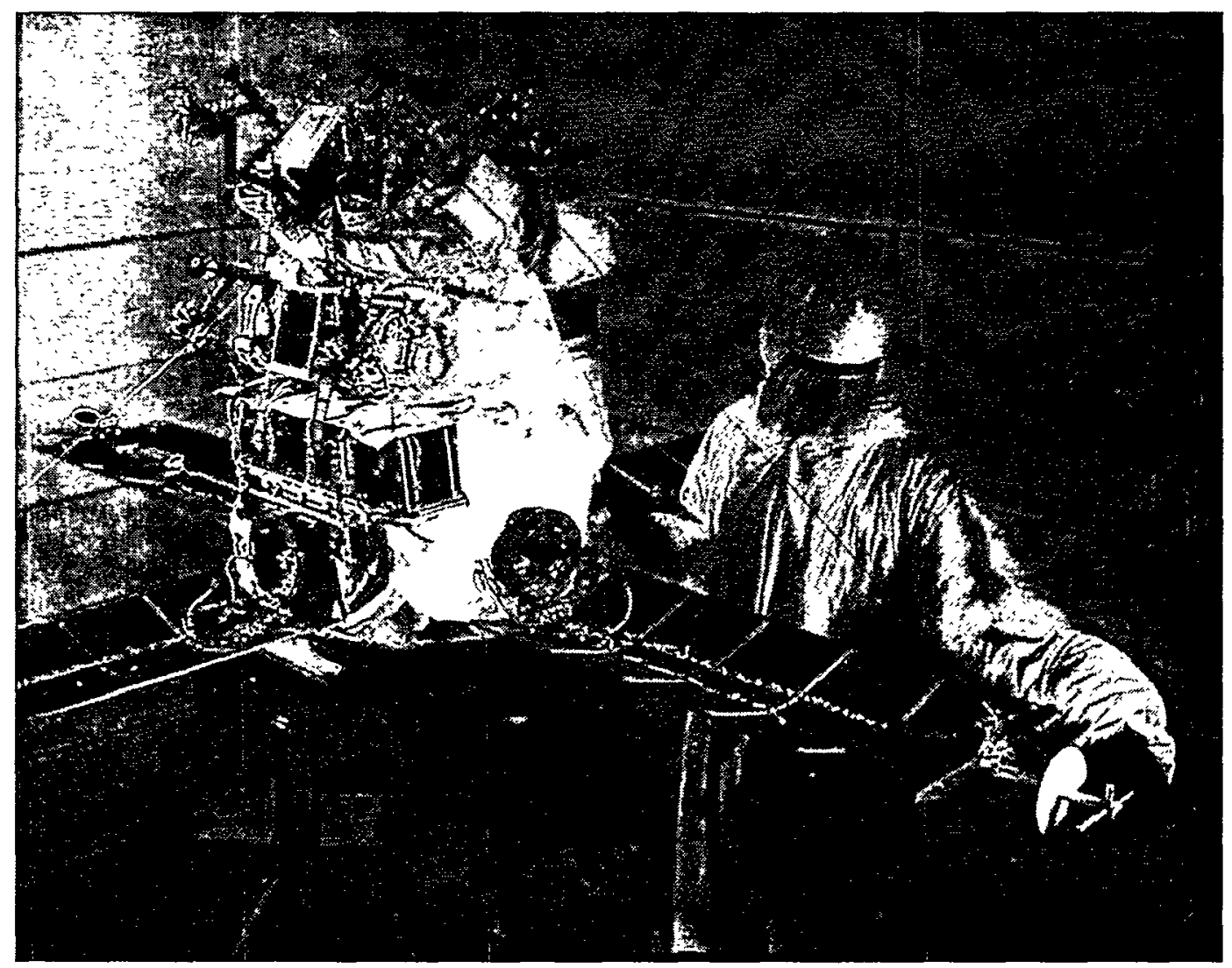

Figure 2.12: A photograph of the ALEXIS satellite taken prior to its launch in 1993. The Blackbeard antennas cannot be seen in the photo, but extend from the top and the bottom of the spacecraft. In orbit, the bottom side of the satellite is sunward-pointing. 


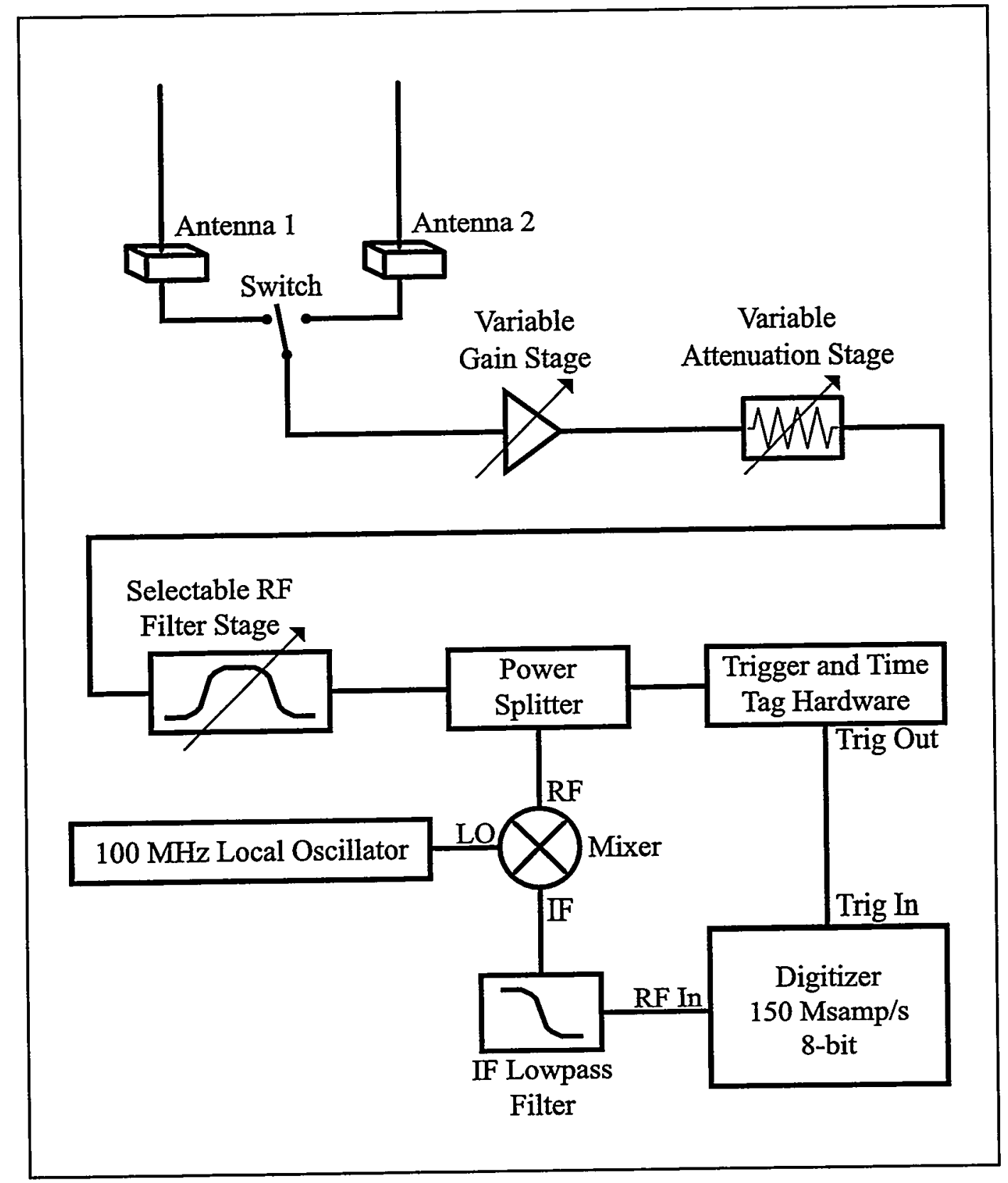

Figure 2.13: A simplified block diagram of the Blackbeard receiver and data acquisition system. 
sixteen in the high band. The local oscillator for the mixing stage is stationary at 100 $\mathrm{MHz}$ and the digitizer samples the intermediate stage at $150 \mathrm{Msamp} / \mathrm{s}$, so the low band and high bands extend from 25 to $100 \mathrm{MHz}$ and $100 \mathrm{MHz}$ to $175 \mathrm{MHz}$ respectively. In actuality, though, the front end RF filters in their most wide open settings restrict the bandwidths to 28 to $95 \mathrm{MHz}$ and 108 to $166 \mathrm{MHz}$ respectively. An IF (intermediate frequency) filter on the mixer output prevents aliasing in the Blackbeard digitizer. The digitizer samples eight bits at $150 \mathrm{Msamp} / \mathrm{s}$. The instrument has 16 Mbyte of memory, corresponding to $112 \mathrm{~ms}$ of total recording time. Memory can be partitioned into 1 Mbyte segments, allowing up to sixteen $7 \mathrm{~ms}$ records to be acquired between downloads. The receiver is triggered by a simple level trigger. Timed collections can be performed by setting the trigger level to zero and arming the instrument at the desired trigger time. Timed collections may be characterized by a jitter of up to a couple tens of milliseconds, but time tags are accurate to within one millisecond, as described in the next section. Blackbeard assigns time tags to all triggers based on its on-board clock. Each trigger receives an MET (mission elapsed time) time stamp, which is converted to UTC when the data are downloaded. Further details regarding the Blackbeard instrument and subsequent data acquisition were described by Holden et al. [1995], Massey and Holden [1995], Smith et al. [1997, a], and Massey et al. [1998].

ALEXIS orbits the earth with a period of approximately 100 minutes. The satellite is typically visible from its ground station at LANL for two or three consecutive passes twice per day. During the summer of 1996 the ground-based electric field change and HF arrays were operated in conjunction with Blackbeard arm 
times over North America. The goal of the simultaneous operations was to make coincident observations of radio emissions using both the ground-based and spacebased resources. Successes were achieved on two days in September of 1996 when Blackbeard TIPP events were recorded in conjunction with ground-based HF recordings (that were triggered by an electric field change meter). Blackbeard waveforms and time stamps were important in the subsequent determinations of the source locations and source phenomenology. As will be shown in Chapter 5, the data provided final, conclusive evidence that TIPP second pulses result from reflections from the surface of the earth.

\subsubsection{Blackbeard Timing Calibration}

In order to utilize Blackbeard time stamps to determine source locations based on differential times of arrival at multiple sensors, it was necessary to calibrate the timing of the system and to characterize its timing accuracy. These issues were addressed in a calibration study performed during November and December of 1996. During the study, the Los Alamos Portable Pulser (LAPP) was used to transmit calibration pulses to Blackbeard. LAPP is pictured in Figure 2.14. The LAPP facility was established in 1991 and transmits an EMP signal whose VHF component is similar to that produced by a nuclear detonation. The energy source for the transmitter is a marx bank generator that produces a $1.2 \mathrm{MV}$ pulse at the feed to a broadband bi-planar dipole antenna that sits at the focus of a $13 \mathrm{~m}$ steerable dish. The duration of the emitted pulse is less than $20 \mathrm{~ns}$ and the peak pulse power is $\sim 1 \mathrm{GW}$ in 


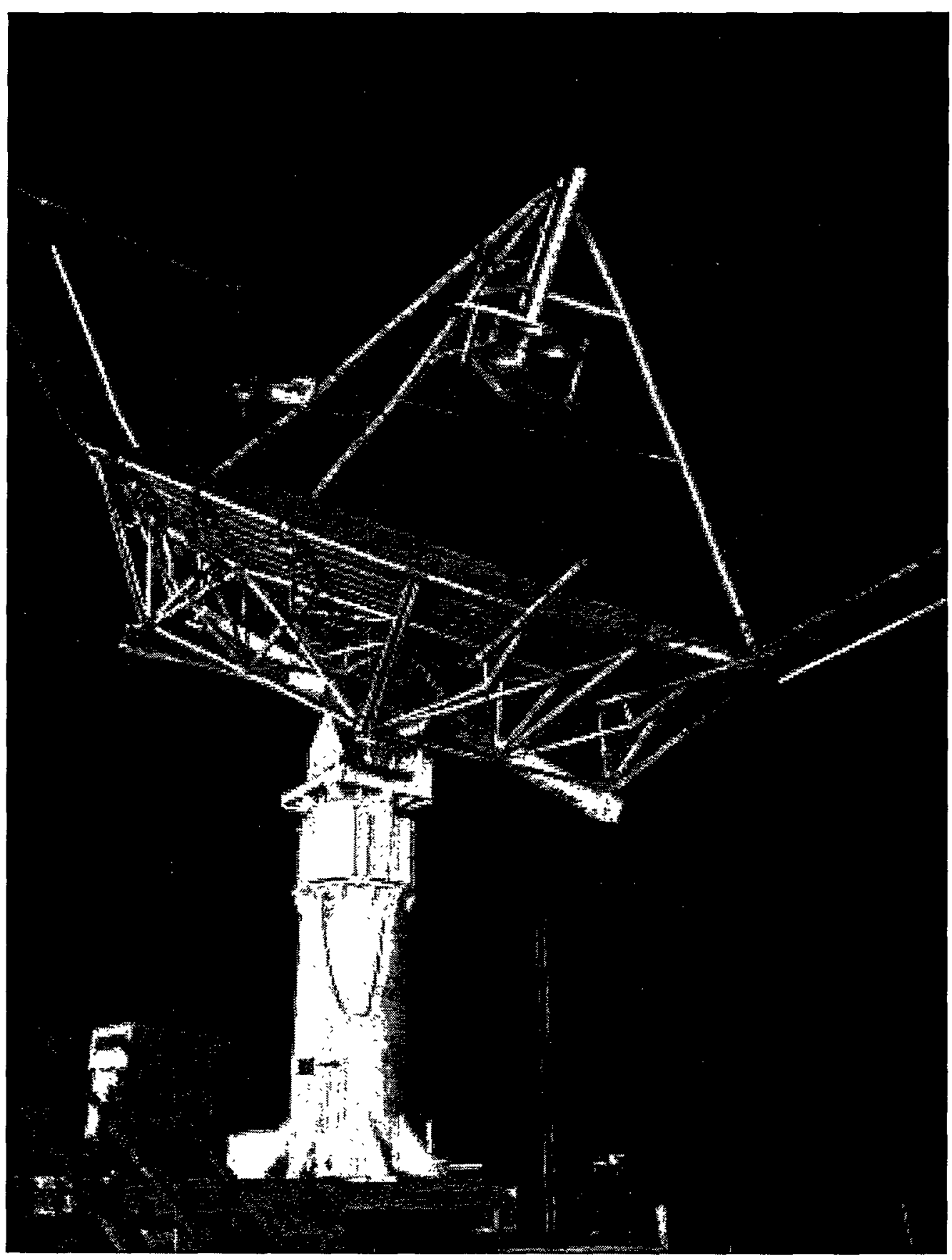

Figure 2.14: A photograph of the Los Alamos Portable Pulser (LAPP). A marx bank generator at the feed of the $13 \mathrm{~m}$ steerable dish simulates the electromagnetic pulse from a nuclear detonation. Transmissions from LAPP were used to calibrate and characterize Blackbeard's time tagging capability. 
a $100 \mathrm{MHz}$ bandwidth. A waveform and spectrogram of a LAPP pulse are shown in Figure 2.15. During the two month study period, LAPP transmitted 31 broadband pulses that were received by Blackbeard.

LAPP firing times were used in conjunction with calculated propagation delays to compute estimated times of arrival (ETOAs) for pulses reaching Blackbeard. The ETOAs were compared to Blackbeard reported times of arrival (RTOAs), which were computed using information returned by Blackbeard and a timing algorithm that was developed specifically for this purpose. For the 31 pulser shots received by Blackbeard, the mean difference between ETOA and RTOA was $1.97 \mathrm{~ms}$ with ETOAs occurring earlier than RTOAs. The distribution of the timing differences is shown in Figure 2.16. The standard deviation of the difference was $0.43 \mathrm{~ms}$. As a result of the study, a $1.97 \mathrm{~ms}$ offset was added to the time tag algorithm, and $0.43 \mathrm{~ms}$ was adopted as a measurement of the Blackbeard timing uncertainty. The calibration study was discussed in detail in Smith et al. [1997, a]. 
Time Series for e10178
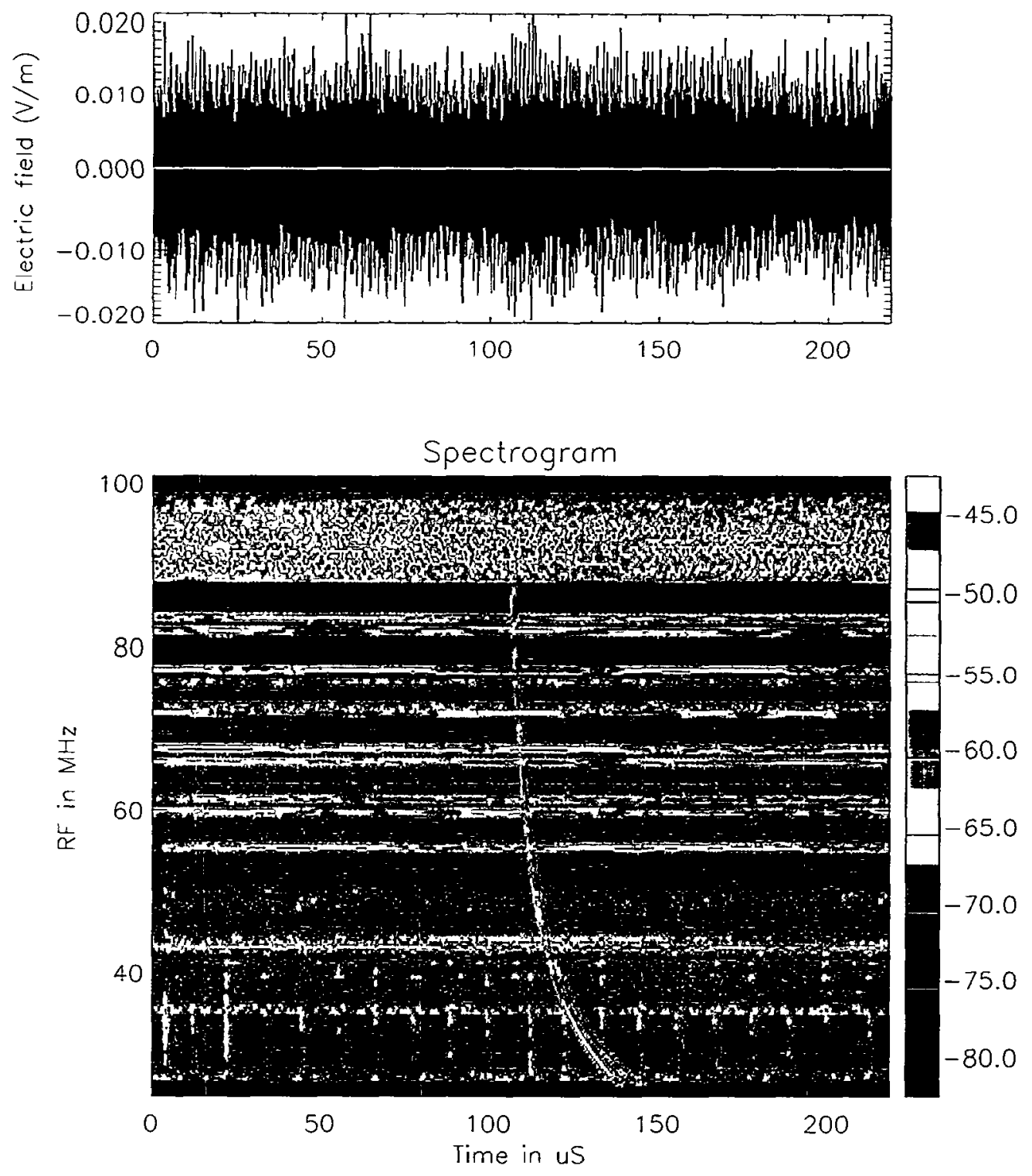

Figure 2.15: An electric field waveform and spectrogram of an electromagnetic pulse transmitted by LAPP and recorded by Blackbeard. At the source the duration of the pulse was less than $20 \mathrm{~ns}$ and the peak pulse power was $\sim 1 \mathrm{GW}$ in a $100 \mathrm{MHz}$ bandwidth. 


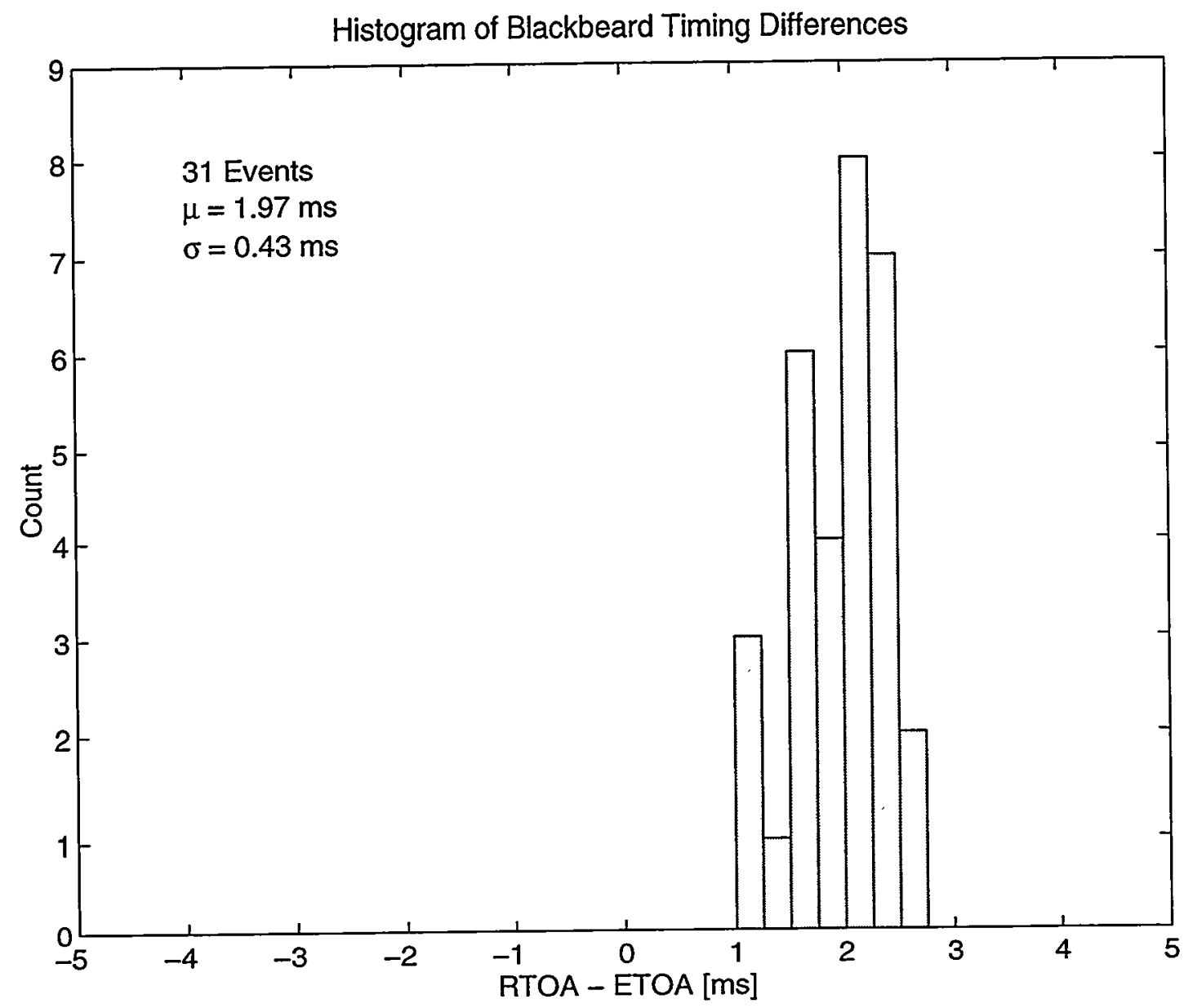

Figure 2.16: A histogram of timing differences recorded during the Blackbeard timing calibration study. 


\section{CHAPTER 3}

\section{SOURCE LOCATION METHODOLOGY}

A variety of methods have been used by researchers to locate lightning flashes and flash components. Among the methods have been techniques based on measurements of electric field amplitudes, magnetic field direction, electric field time of arrival, electric field interferometry, visible light direction, thunder time of arrival, and radar echoes. Uman $[1987, \mathrm{c}]$ provided a review of these techniques.

In this study, long baseline time of arrival techniques were used to locate the sources of electric field change and radio frequency signals in three dimensions. Long baseline refers to the fact that the distances between sensors represented a significant fraction of the distances to the sources being located. The concept of using time of arrival techniques to locate lightning was first published by Oetzel and Pierce $[1969$, b]. The authors described short baseline location techniques, whereby differential times of signal arrival at stations separated by a few hundred meters could be used to determine bearings to signal sources at ranges of many tens of kilometers. Short baseline systems do not provide the high accuracy that is characteristic of long baseline systems, but simplify the task of sorting out signals received by multiple stations from different locations. Because lightning signals are often received as bursts of pulses, it can be difficult or impossible to make one-to-one correlations 
between the individual pulses received by different stations. This task becomes more difficult as receiver baselines are extended. Before the advent of relatively inexpensive and simple methods of determining absolute time tags to a high degree of accuracy (e.g. through the use of GPS receivers), short baseline time of arrival systems were simpler to implement than long baseline systems.

Proctor [1971, 1981] performed the pioneering work on lightning location using long baseline time of arrival techniques. He used five VHF ground stations separated by distances ranging from 10 to $30 \mathrm{~km}$ to record and time tag lightning emissions that occurred within many tens of kilometers of his recording stations in South Africa. He used the differential times of arrival (DTOAs) between station pairs to form isochrones in a Cartesian coordinate system. These isochrones are hyperboloids along which constant DTOA relationships exist between station pairs. For a 2-D illustration of such isochrones refer to Figure 3.1. The figure shows three stations labeled X, Y, and $\mathrm{Z}$, and three isochrones labeled $\mathrm{XY}, \mathrm{YZ}$, and $\mathrm{ZX}$. The source location for an event (labeled S) is at the intersection of the isochrones formed from the measured differential times of arrival. If the lines do not intersect at a single point due to station threshold differences or other factors, an error minimization routine is implemented to determine the most likely source location and evaluate the location uncertainty. Proctor [1971] used his system to determine 3-D source locations. Because his vertical measurement baselines were short compared to his horizontal measurement baselines, the uncertainty in his determinations of source altitude was about five times the uncertainty in his determinations of source locations in $\mathrm{X}$ and $\mathrm{Y}$. Although larger 


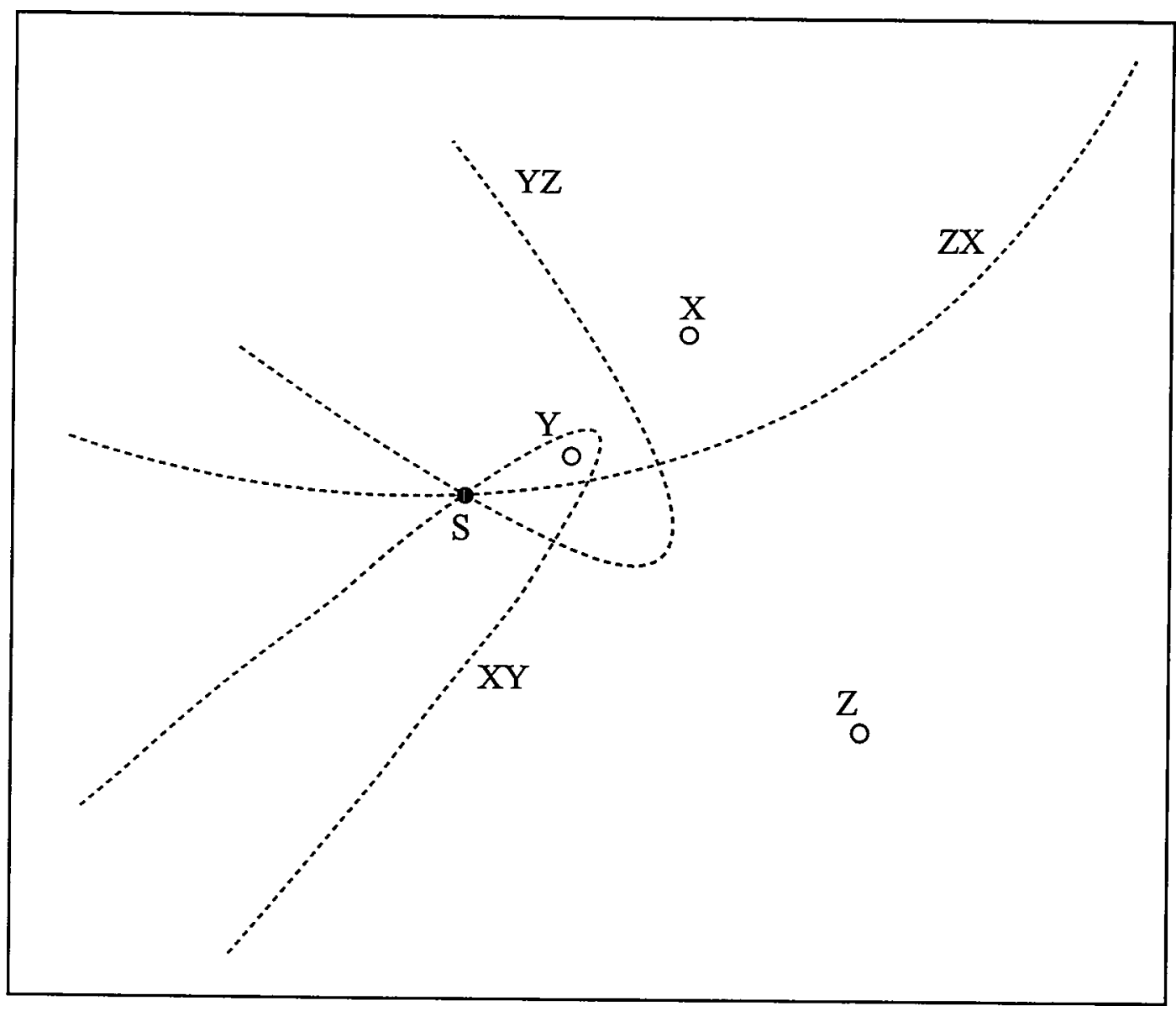

Figure 3.1: Illustration of isochrones in two dimensions. The isochrones (dotted lines) represent constant DTOA relationships between station pairs. Their intersection shows the location of a source (S). The stations are $\mathrm{X}, \mathrm{Y}$, and $\mathrm{Z}$. The pairs are $\mathrm{XY}, \mathrm{YZ}$, and ZX. 
than the horizontal location uncertainty, the vertical location uncertainty was still quite good, being on the order of $0.5 \mathrm{~km}$.

To locate lightning sources in three dimensions, techniques similar to those used by Proctor were utilized in the study described here, but with an important additional feature. Reflections of signals from the ionosphere and earth were used to form virtual vertical measurement baselines. The virtual baselines made it possible to determine accurate source heights for elevated events that occurred at distant ranges. Figure 3.2 shows the typical propagation paths (solid black lines) that were received from elevated electric field change sources by the electric field change meters. In many cases HF signals were also received along similar paths. In Figure 3.2, path A is the direct path from the elevated source to a ground-based receiver. Path B is the 1hop reflection path from the ionosphere. Path $\mathrm{C}$ is the 1-hop reflection path that reflected first from the surface of the earth and then from the ionosphere. The height of the source in the figure is $h$, the virtual reflection height of the ionosphere is $H$, and the range from the source to the receiver is $r$. In utilizing the times of arrival of the reflected signals to assist in the determinations of source locations, the signals were treated as if they had been received by image receivers located above the ionosphere and below the surface of the earth. This is because the signal propagation path lengths to the image receivers were the same as those actually followed by the reflected signals. The locations of the image receivers are shown in Figure 3.2, along with the virtual propagation paths (dashed gray lines). The figure provides a visual illustration of the improvement in the vertical baseline provided by the method. 


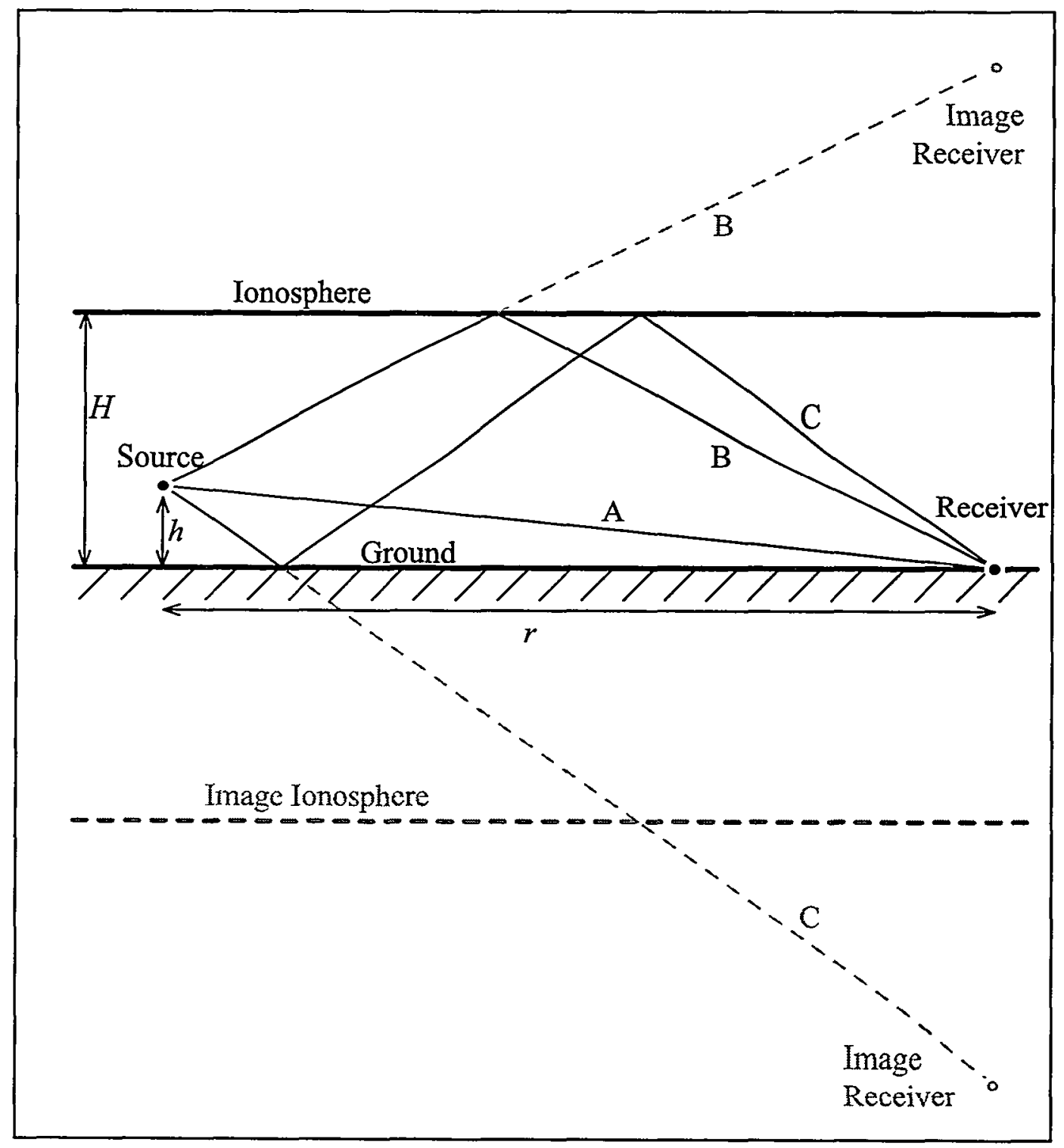

Figure 3.2: $\quad$ Illustration of propagation paths and virtual receiver locations. Solid lines $A, B$, and $C$ show propagation paths from a source to a receiver. Path $A$ is a groundwave or line-of-sight path. Paths $B$ and $C$ are 1-hop skywave paths that propagate via reflections from the ionosphere. Dotted lines show paths to images of the receivers. The concept of image receivers was critical in the determination of accurate source altitudes. 
As a simple quantitative demonstration of the improvement, consider that source heights $(h)$ were typically $8 \mathrm{~km}$, ionosphere heights $(H)$ were typically $90 \mathrm{~km}$ for reflections from the E-layer ( $H$ was over $250 \mathrm{~km}$ for reflections from the F-layer, but such reflections were less commonly observed), and source ranges $(r)$ were between 80 and $460 \mathrm{~km}$. The source height uncertainties for actual events were between \pm 0.3 and $\pm 0.8 \mathrm{~km}$ based on timing uncertainties of 2 to $5 \mu \mathrm{s}$. Three-dimensional locations for these events were determined using TOAs from between two and five stations. Times of arrival of reflected signals were also utilized. If the reflection data had not been used (or had not been available due to less favorable ionospheric conditions), then at least four stations would have been necessary to determine 3-D locations and the height uncertainties would have been on the order of tens of $\mathrm{km}$ for the same timing uncertainties. Thus source height determinations would have been useless without the ionospheric reflections.

In order to use the times of arrival of the reflected signals, it is necessary to either know a priori the virtual height of the ionosphere or to calculate the virtual height using recorded data. For all of the events described in this dissertation, enough information was available from multiple sensors and multiple reflections to compute $H$ from the data alone.

The relative timing of reflections of electric field change signals from the ionosphere has previously been used by Laby et al. [1940], Kessler and Hersperger [1952], Kinzer [1974], and McDonald et al. [1979] to locate signal sources. These researchers used the timing of multiple hop reflections to determine ranges to lightning ground strokes. Thus the authors studied signals that had their origin at 
ground level. There appears to be no mention in the literature of using ionospheric reflections to determine the altitudes of elevated sources. The present application appears to be the first such application.

\subsection{SOURCE LOCATIONS FROM GROUND-BASED MEASUREMENTS}

Times of arrival from between two and five stations were used to determine CD source locations within three southwestern thunderstorms during the summer of 1996. A well-developed, nighttime ionospheric E-layer was present during the times that the storms were observed. As mentioned in the previous section, it would have been impossible to determine source heights without the ionospheric reflections of electric field change and/or broadband HF signals. It is probably not a coincidence that all of the observations were made at night. Electric field change signals are VLF/LF radio waves and suffer absorption from the D-region during the daytime. During four years of measurements, it has been observed that good reflections of electric field change signals are most often recorded after sundown, especially after about 22:00 local time. It has also been observed that groundwave signals tend to propagate further during the night. These observations are consistent with decay of the D-region after sundown due to recombination in the upper atmosphere.

Reflections from the ionosphere and the surface of the earth provided image receivers located directly above and below the actual receiving stations as illustrated in Figure 3.2. In order to use the information from the image receivers, 
simplifications were made regarding the propagation of radio signals from the sources to the receivers and regarding reflections from the ionosphere and ground. The radio signals were assumed to follow the rules of geometric optics. Thus signals were assumed to propagate in straight lines and ideal reflections were assumed to take place from the ground and from the virtual reflection height of the ionosphere. By definition, the virtual reflection height is the height from which a signal appears to reflect under the laws of geometric optics. In reality, reflections from the ionosphere are not true reflections. Radio waves are actually refracted back to earth from the ionosphere and the virtual reflection height is higher than the actual maximum height attained by radio signals [Davies, 1990]. Descriptions of the refraction processes become much more complex if horizontal electron density gradients, electron density inhomogeneities, and the earth's magnetic field are taken into account. Fortunately, the characteristics of the E-layer lend well to its being treated as a geometric reflector. The E-layer, which is typically centered at an altitude of $100 \mathrm{~km}$, acts much like a mirror for radio waves with wavelengths longer than the density gradient scale length. This is certainly true for VLF/LF transmissions and approximately true for HF. All location techniques were based on differential times of arrival and all solutions were found using techniques of error minimization. That is, source locations were simply moved in latitude, longitude, and altitude until the location was found that most closely reproduced the recorded DTOAs. Thus it was important to determine sets of equations that properly described the relative timing relationships as a function of the source and receiver positions for every different case. Each set of 
equations was solvable as long as the number of independent equations was greater than the number of unknowns (and the equations were reasonably well behaved).

For the first and third storms that were studied, at least three spatially separated recording stations recorded events of interest. Spatial separation is required because co-located stations do not provide independent timing data. The FC1 and HF1 stations were co-located at LANL. For the events recorded during storms 1 and 3, three dimensional source locations were determined using a two step process. Because three or more independent stations time tagged events during the storms, it was possible to first determine 2-D source locations (latitude and longitude) and then determine source altitudes and ionospheric virtual heights in a second step using the reflection delays. For the second storm, only two spatially separated stations recorded events. For these events, latitude, longitude, altitude, and ionospheric virtual height were determined in a single step by simultaneously solving the equations that described the DTOA relationships. The following sections describe the two-step and one-step location methods in detail.

\subsubsection{Location Method One: Three or More TOA Receivers}

For each $\mathrm{CID}$ recorded during storms 1 and 3 , at least three receivers recorded time tags. For these events, 3-D source locations were determined in two steps. The first step was to find the latitude and longitude of the source (2-D location) as determined from multiple station differential times of arrival of the groundwave signals (for field change) or line-of-sight signals (for HF). These determinations were 
accomplished by systematically computing the expected differential times of arrival at the actual stations over a coarse grid of source location guesses. The point on the grid at which the calculated DTOAs most closely matched the measured DTOAs for the actual event was determined. This point was used as the center of a smaller, finerresolution grid of location guesses. The magnification procedure was repeated iteratively until the best estimate of the source location with the desired spatial precision was determined. Since 2-D location uncertainties were rarely less than a few hundred meters, this was usually the approximate precision obtained. This step of the location procedure was an exercise in minimizing the summed square errors between actual measured DTOAs and expected DTOAs computed for locations on a grid of increasingly finer resolution. For each guess on a grid, the expected DTOAs were computed as in the following example.

Figure 3.3 shows three TOA recording stations $\left(R_{1}, R_{2}\right.$, and $\left.R_{3}\right)$ and a source ( $S$ ) that produced a signal that was recorded by all three stations. The figure also shows a guess $(G)$ at the location of the source, the point for which the expected DTOAs in this example are to be computed. In order to determine the expected differential times of signal arrival between stations $R_{1}$ to $R_{2}, R_{2}$ to $R_{3}$, and $R_{3}$ to $R_{1}$, it is necessary to determine how long it takes the signal to propagate to each station from the location of the guess, G. In this step it is assumed that the earth is a sphere and that the signals propagate from their source to the receivers via great circle propagation paths. The arc distance, $r_{G C}$, between two points on the surface of the earth is given by the following equation: 


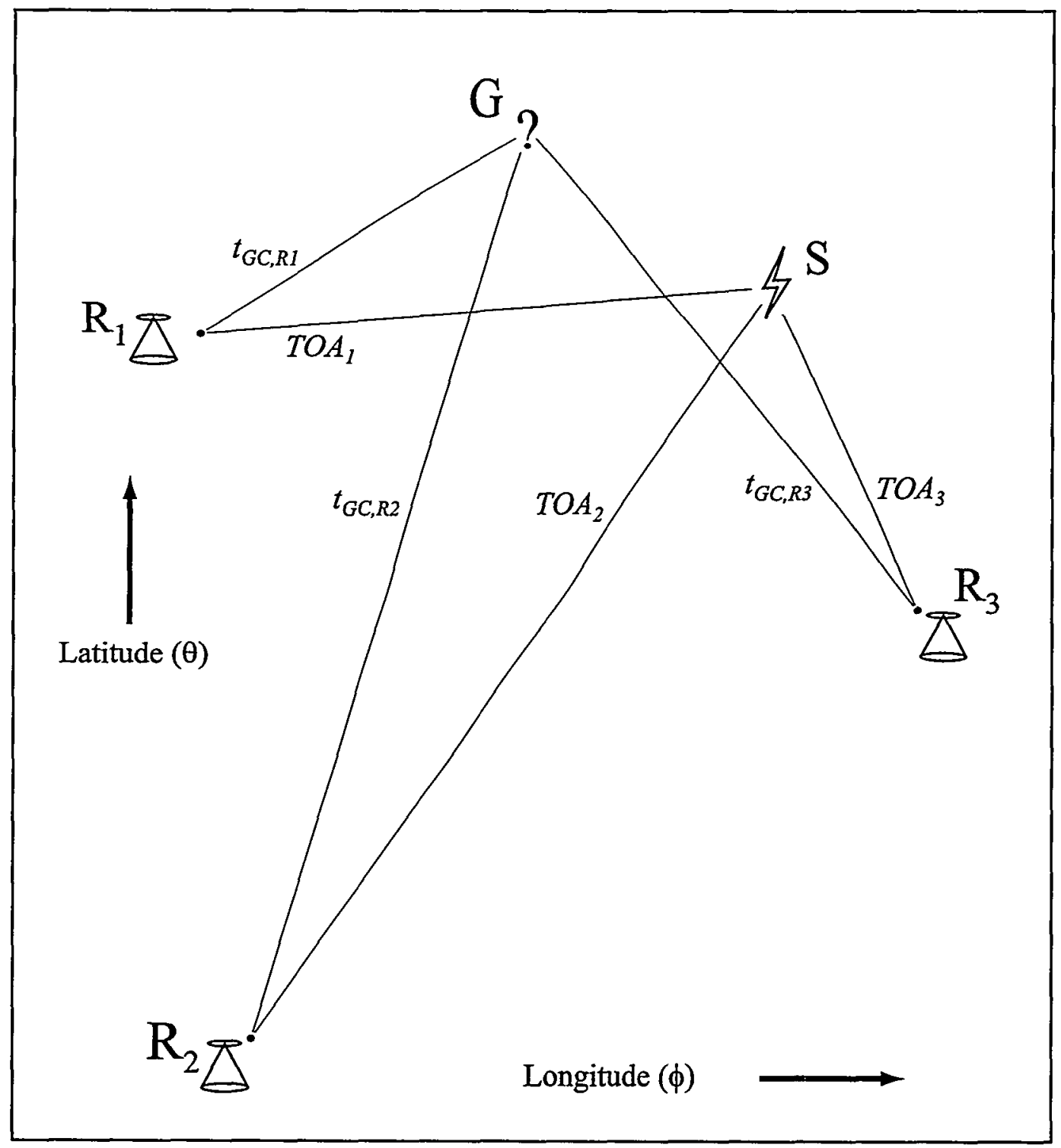

Figure 3.3: Ilustration of the method used to locate sources in latitude and longitude. Differences between $t_{G C}$ values and TOA values were minimized to determine the guess location (G) that most closely matched the actual source location (S). 


$$
r_{G C}=r_{e} \cos ^{-1}\left[\sin \left(\theta_{R}\right) \sin \left(\theta_{G}\right)+\cos \left(\theta_{R}\right) \cos \left(\theta_{G}\right) \cos \left(\phi_{R}-\phi_{G}\right)\right]
$$

where $r_{e}$ is the radius of the earth, $\theta_{R}$ and $\phi_{R}$ are the latitude and longitude of the recording station under consideration, and $\theta_{G}$ and $\phi_{G}$ are the latitude and longitude of the source guess location.

The time required $\left(t_{G C}\right)$ for the signal to propagate along the great circle path from the source guess location to the given recording station is given by the following relation:

$$
t_{G C}=\frac{r_{G C}}{c}
$$

where, as in previous chapters, $c$ is the speed of light.

The expected TOA $\left(t_{G C}\right)$ is computed at each receiver for each source guess location. In this example the TOAs are represented as $t_{G C, R I}, t_{G C, R 2}$, and $t_{G C, R 3}$. The differences between the expected TOAs are represented as $d t_{1,2}=t_{G C, R I}-t_{G C, R 2}, d t_{2,3}=$ $t_{G C, R 2}-t_{G C, R 3}$, and $d t_{3,1}=t_{G C, R 3}-t_{G C, R I}$. These differences are compared to the actual measured differential times of arrival: $D T O A_{1,2}=T O A_{1}-T O A_{2}, D T O A_{2,3}=T O A_{2}$ $T O A_{3}$, and $D T O A_{3,1}=T O A_{3}-T O A_{1}$. The summed square error (SSE) between the estimated TOAs for the location guess and the actual TOAs is given by:

$$
S S E=\left(\operatorname{DTOA}_{1,2}-d t_{1,2}\right)^{2}+\left(\operatorname{DTOA}_{2,3}-d t_{2,3}\right)^{2}+\left(D \operatorname{TOA}_{3,1}-d t_{3,1}\right)^{2}
$$


The summed square error value is computed for every point on each grid of source guess locations. The point with the minimum summed square error is the best location estimate for the grid. The procedure is repeated for finer resolution grids centered on the best location estimates until the desired location resolution is attained. The grid magnification technique was used because it was computationally faster than starting with a grid of the desired resolution that had the same spatial size as the initial coarse grid.

Once the 2-D location of an event was determined, the source altitude was determined using the delays of the reflected signals (see paths B and C in Figure 3.2) relative to that of the direct path (path A). For storm 1, only field change reflections were used to determine source heights because no HF reflections were recorded from the source. For storm 3, both field change and HF reflections were used to determine source heights. Because the ionosphere virtual heights were different for the two frequency ranges of signals (as will be shown in Chapter 4), the computations were performed independently of one another.

For each receiving station that recorded the reflected signals, a source height estimate $(h)$ and ionospheric virtual height estimate $(H)$ were made based on the range to the source $(r)$ and the difference in the times of arrival from the direct pulse to the two reflected pulses. Again, geometric optics were assumed to describe the paths of the reflected signals. In this step, a flat earth propagation model was used to simplify the computations of the path delays. The simplification caused very small errors in the computations of source heights. Even for the most distant events, that occurred at ranges of over $400 \mathrm{~km}$, the error in the source height calculation as a result of using 
the flat earth model was less than $10 \mathrm{~m}$. This error was much smaller than the height uncertainties, which were at least a few hundred meters.

The lengths of paths $\mathrm{A}\left(P_{A}\right), \mathrm{B}\left(P_{B}\right)$, and $\mathrm{C}\left(P_{C}\right)$ in Figure 3.2 are given by:

$$
\begin{aligned}
& P_{A}=\sqrt{r^{2}+h^{2}} \\
& P_{B}=\sqrt{r^{2}+(2 H-h)^{2}} \\
& P_{C}=\sqrt{r^{2}+(2 H+h)^{2}}
\end{aligned}
$$

The differences between the path lengths are related to the measured differential delays, $t_{1}$ and $t_{2}$, by the following relations:

$$
\begin{aligned}
& P_{B}-P_{A}=t_{1} c \\
& P_{C}-P_{A}=t_{2} c
\end{aligned}
$$

Substituting Equations 3.4 through 3.6 into Equations 3.7 and 3.8, two equations with two unknowns, $h$ and $H$, remain. These equations were solved simultaneously using a minimum mean square error technique similar to that used in step one of the location procedure. Independent height estimates were computed for each station that recorded ionospheric and ground reflections. 
Uncertainty in the determinations of source locations depended on the timing uncertainties of the recording stations and the positions of the stations relative to each other and the source. Two-dimensional location uncertainties were computed by systematically varying the actual time tags from stations by their respective timing uncertainties, solving for the source locations, and comparing the new source locations to the original source locations. Computations of height uncertainties for location method one depended on the accuracy with which it was possible to determine the differential times of arrival between the direct signals and their reflections from the ionosphere, and also on the uncertainty in the 2-D source location. Two-dimensional uncertainties ranged from $\pm 200 \mathrm{~m}$ to $\pm 20 \mathrm{~km}$. Height uncertainties ranged from \pm 0.3 to $\pm 0.8 \mathrm{~km}$. More precise numbers will be presented in Chapter 4.

\subsubsection{Location Method Two: Two TOA Receivers}

The location method described in the previous section was used to determine the 3-D locations of $\mathrm{CD}$ s that occurred during storms 1 and 3 . During storm 2 only two spatially separated time-of-arrival receivers recorded $\mathrm{CID}$ electric field change and HF emissions, so it was not possible to use step one from the previous procedure to first determine the 2-D source location. Instead, steps one and two were combined to determine 3-D source locations in a single step. Note that to use this procedure it was necessary to have recorded reflections from both the earth and ionosphere at both of the recording stations. 
As was the case in the previous method, it was assumed that reflections from the earth and ionosphere obeyed the rules of geometric optics. Also the flat earth/ionosphere model was utilized in order to simplify the relationships between the variables. Errors resulting from the simplification were on the order of $10 \mathrm{~m}$, a number much smaller than the measurement uncertainty. It was further assumed that the ionospheric virtual heights were the same for both of the propagation paths and that the heights of the source and ionosphere above ground level were the same as viewed from both recording stations.

Using this method, it was necessary to solve for four variables simultaneously in order to determine each of the source locations. The variables were: $h$, the height of the source above ground level; $H$, the virtual height of the ionosphere above ground level; $r_{l}$, the range to the source from the first station; and $r_{2}$, the range to the source from the second station. In general, if these variables are solved for, there are actually two possible source locations for each event. For storm 2, the ambiguity was resolved by comparing locations to lightning location data reported by an independent resource, the National Lightning Detection Network (NLDN). The NLDN is described in some detail in later chapters, but basically provides the locations of cloud-to-ground lightning strokes to its customers. During storm 2 the $\mathrm{CD}$ source region was determined to be either in Texas near the southeastern corner of New Mexico, or in southeastern Utah. The NLDN did not report any lightning activity in the region in Utah, but reported intense cloud-to-ground lightning activity in the region in Texas (as will be shown in Chapter 4). The CIDs were assumed to occur in 
the region of other thunderstorm electrical activity and the ambiguity was thus resolved.

A system of equations was used to solve for the variables $\left(h, H, r_{1}\right.$, and $\left.r_{2}\right)$ using the differential times of arrival between all of the signals recorded by the two stations. The following equations describe the relationships between the unknown variables and the measured differential time delays:

$$
\begin{aligned}
& \sqrt{r_{1}^{2}+h^{2}}-\sqrt{r_{2}^{2}+h^{2}}=t_{0} c \\
& \sqrt{r_{1}^{2}+(2 H-h)^{2}}-\sqrt{r_{1}^{2}+h^{2}}=t_{11} c \\
& \sqrt{r_{1}^{2}+(2 H+h)^{2}}-\sqrt{r_{1}^{2}+h^{2}}=t_{12} c \\
& \sqrt{r_{2}^{2}+(2 H-h)^{2}}-\sqrt{r_{2}^{2}+h^{2}}=t_{21} c \\
& \sqrt{r_{2}^{2}+(2 H+h)^{2}}-\sqrt{r_{2}^{2}+h^{2}}=t_{22} c
\end{aligned}
$$

The differential times of arrival $\left(t_{0}, t_{11}, t_{12}, t_{21}\right.$, and $\left.t_{22}\right)$ correspond to differences between paths shown in Figure $3.4\left(P_{1 A}-P_{2 A}, P_{1 B}-P_{1 A}, P_{1 C}-P_{1 A}, P_{2 B}-P_{2 A}\right.$, and $P_{2 C}-P_{2 A}$ respectively). The equations were solved simultaneously using an error minimization routine written by Xuan-Min Shao of LANL. The routine used a gradient search to 


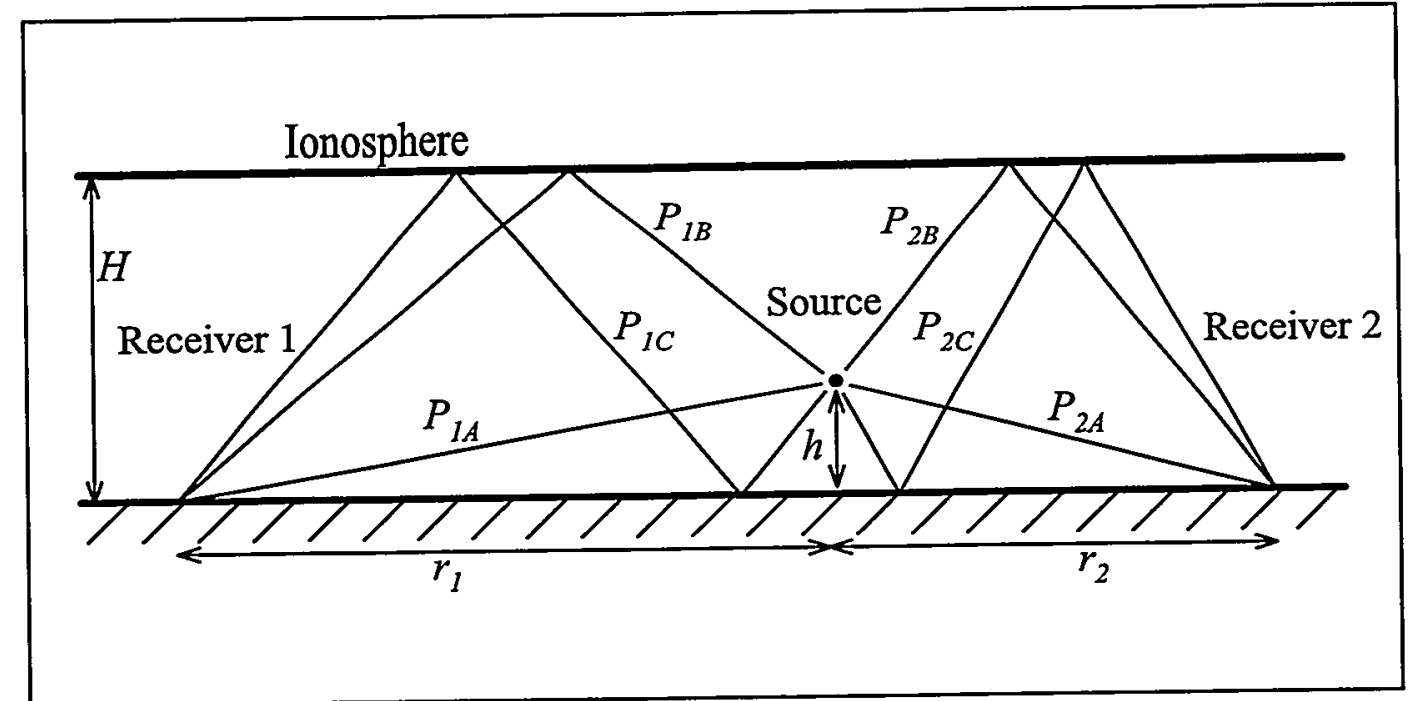

Figure 3.4: Illustration of the propagation paths used to determine $h, H, r_{1}$, and $r_{2}$ using location method two. 
determine the combination of variables that resulted in the minimum error. Threedimensional location uncertainties were determined by systematically varying the times of arrival of the direct and reflected pulses by their respective timing uncertainties and recomputing the source locations.

\subsubsection{Other Location Notes}

The differences in the altitudes of the recording stations were not taken into account in the previously described location methods. A simplification was used and it was assumed that the stations were all in the same plane at the altitude of the reflection point of the signal. The simplification introduced small errors in the 3-D source locations. The horizontal error in the worst case (close events recorded by FC2 and FC3, which were separated in altitude by nearly $2 \mathrm{~km}$ ) was $10 \mathrm{~m}$. The vertical error was $20 \mathrm{~m}$. Both of these uncertainties are much smaller than the relative uncertainties.

Since all of the measurements were relative to ground level, source and ionosphere altitudes were determined above ground level. In order to convert the AGL values to altitudes above mean sea level (MSL), USGS (United States Geological Survey) maps were consulted to determine the elevations at the approximate reflection points for each of the three storms. Since 3-D source locations were known, it was possible to determine the locations of the reflection points to within the accuracy of the 2-D source locations themselves. The elevations for the three storms were $1.8,1.2$, and $2.1 \mathrm{~km}$ MSL respectively. Throughout the remainder 
of the dissertation, these offsets will be applied to convert AGL values to MSL. MSL values are more pertinent to determining meteorological conditions, such as isotherm heights, than AGL values.

\subsection{SOURCE LOCATIONS FROM GROUND- AND SATELLITE-BASED MEASUREMENTS}

On two occasions in September of 1996 coincident observations of RF emissions from CIDs were made using both ground-based receivers and the satellite-borne Blackbeard instrument. As was the case for the ground-based only measurements, methods of differential time of arrival (including the use of image receivers) were used to locate the sources of the events. For these observations, the methodology used to locate the sources was quite specific to the data received by the different sensors. For this reason, the descriptions of the source location methods are left to Chapter 5, where the coincident satellite observations will be described. 


\section{CHAPTER 4}

\section{OBSERVATIONS FROM THREE SOUTHWESTERN THUNDERSTORMS}

The data presented in this chapter were recorded from three nighttime thunderstorms that took place in New Mexico and west Texas during the summer of 1996. Storm 1 took place on July 30 and was centered approximately $170 \mathrm{~km}$ eastnortheast of Los Alamos. Storm 2 took place on August 6 approximately $440 \mathrm{~km}$ southeast of Los Alamos, just east of the border between New Mexico and Texas. Storm 3 took place on August 17 approximately $100 \mathrm{~km}$ south-southeast of Los Alamos. The locations of the three storms and the locations of the LANL and NMIMT receivers are shown in Figure 2.1.

The storms were summertime air-mass thunderstorms. Each was fairly localized, began in the early to late afternoon, and lasted until after 02:00 local time (MDT). Each storm produced large numbers of intracloud and cloud-to-ground lightning strokes. Maximum cloud-to-ground (CG) flash rates for the storms reached 9, 17, and 4 flashes per minute respectively, based on data from the National Lightning Detection Network (NLDN). 


\subsection{El.eCTRIC FIELd CHANGF ANI HF WAVEFORMS FROM CIDS}

What is noteworthy about the three thunderstorms described in this chapter is that each produced distinct, isolated, powerful, short-duration fast electric field change pulses that were accompanied by very powerful HF radio bursts during periods of intense thunderstorm activity. The sources of these emissions were dubbed CIDs in the Introduction. As will be shown in this and subsequent chapters, CID radio emissions were profoundly different from those produced by CG and typical IC (intracloud) lightning strokes that occurred in the same storms.

The electric field change waveform and broadband HF waveform from a CID are shown in Figure 4.1. The event was recorded by the LANL field change and HF systems (FCl and $\mathrm{HF} 1$ ) and occurred during storm 3. The distance from the source to the receivers was $103 \mathrm{~km}$. The height of the source was $9.2 \mathrm{~km} \mathrm{MSL}$. The event was also recorded by the FC2 and FC3 field change systems and was located using method one, which was described in the previous chapter.

The top panel of Figure 4.1 shows the electric field change waveform associated with the event. The distinct, bipolar, initially-positive waveform closely matches NPBPs recorded by Le Vine [1980], Willett et al. [1989], and Medelius et al. [1991] (see Figures 1.9 and 1.10, recalling that Le Vine [1980] used the opposite convention for electric field change polarity). The FWHM of the initial portion of the pulse in Figure 4.1 is $6 \mu \mathrm{s}$. The overall duration is $26 \mu \mathrm{s}$. The rise time is $4 \mu \mathrm{s}$. The peak amplitude of the field change waveform is $4.3 \mathrm{~V} / \mathrm{m}$. 

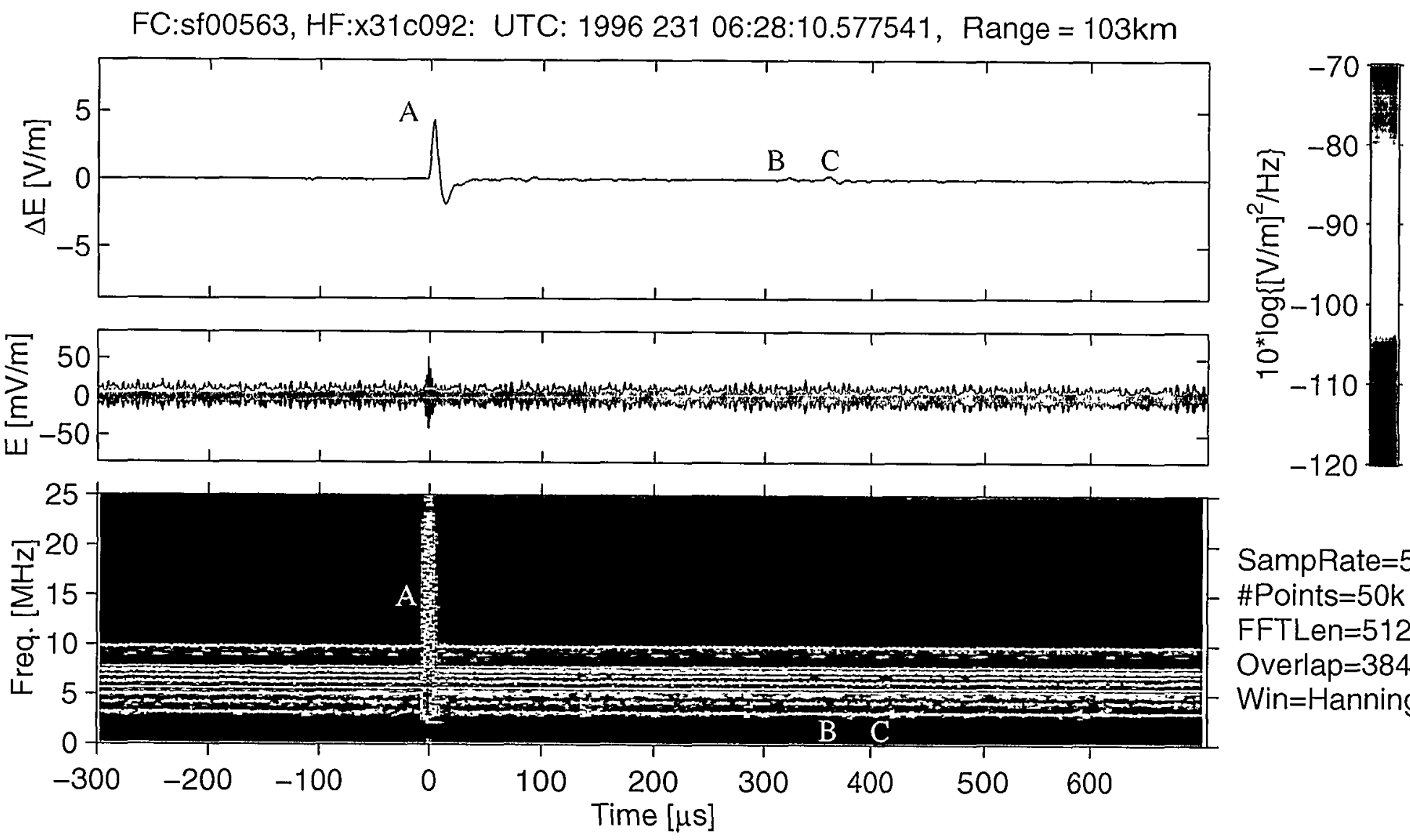

SampRate $=50 \mathrm{M}$

\#Points=50k

FFTLen $=512$

Overlap $=384$

Win=Hanning

Figure 4.1: Electric field change waveform, broadband HF waveform, and HF spectrogram of a CID that occurred during storm 3 . Very weak ionospheric reflections can be seen in the upper and lower panels and are labeled B and C. 
The middle panel of the figure shows the broadband HF electric field waveform from the CID. The duration of the HF radio burst is $2.0 \mu$ s. The peak amplitude is 51 $\mathrm{mV} / \mathrm{m}$. The high background noise level is from communication carriers within the receiver passband. The signal to noise ratio (peak signal power to mean noise power) for the event is $17 \mathrm{~dB}$.

The image in the bottom panel of Figure 4.1 is a time-frequency spectrogram of the broadband HF electric field waveform. The spectrogram was formed using a 512 point $(\sim 10 \mu \mathrm{s})$ sliding Hanning window. Successive waveform segments were overlapped by $3 / 4$. The horizontal lines below $10 \mathrm{MHz}$ are communication signals within the receiver passband.

Several hundred fast electric field change and $\mathrm{HF}$ waveforms with characteristics closely matching those exhibited by the event in Figure 4.1 were recorded by the New Mexico electric field change and HF systems from seven storms at ranges of up to $1800 \mathrm{~km}$ during the summer of 1996 . Accurate locations were obtained only during the three thunderstorms described in this chapter and the tropical cyclone described in Chapter 5.

Both the field change and HF waveforms shown in Figure 4.1 were small in amplitude compared to most of the waveforms recorded from NPBPs during the study. The waveforms did not saturate their respective recording systems. Because NPBPs were some of the largest-amplitude waveforms received from thunderstorms, recordings were often saturated. This was the case for the CID pictured in Figure 4.2 which, like the previous example, was recorded from storm 3 . For this event the 


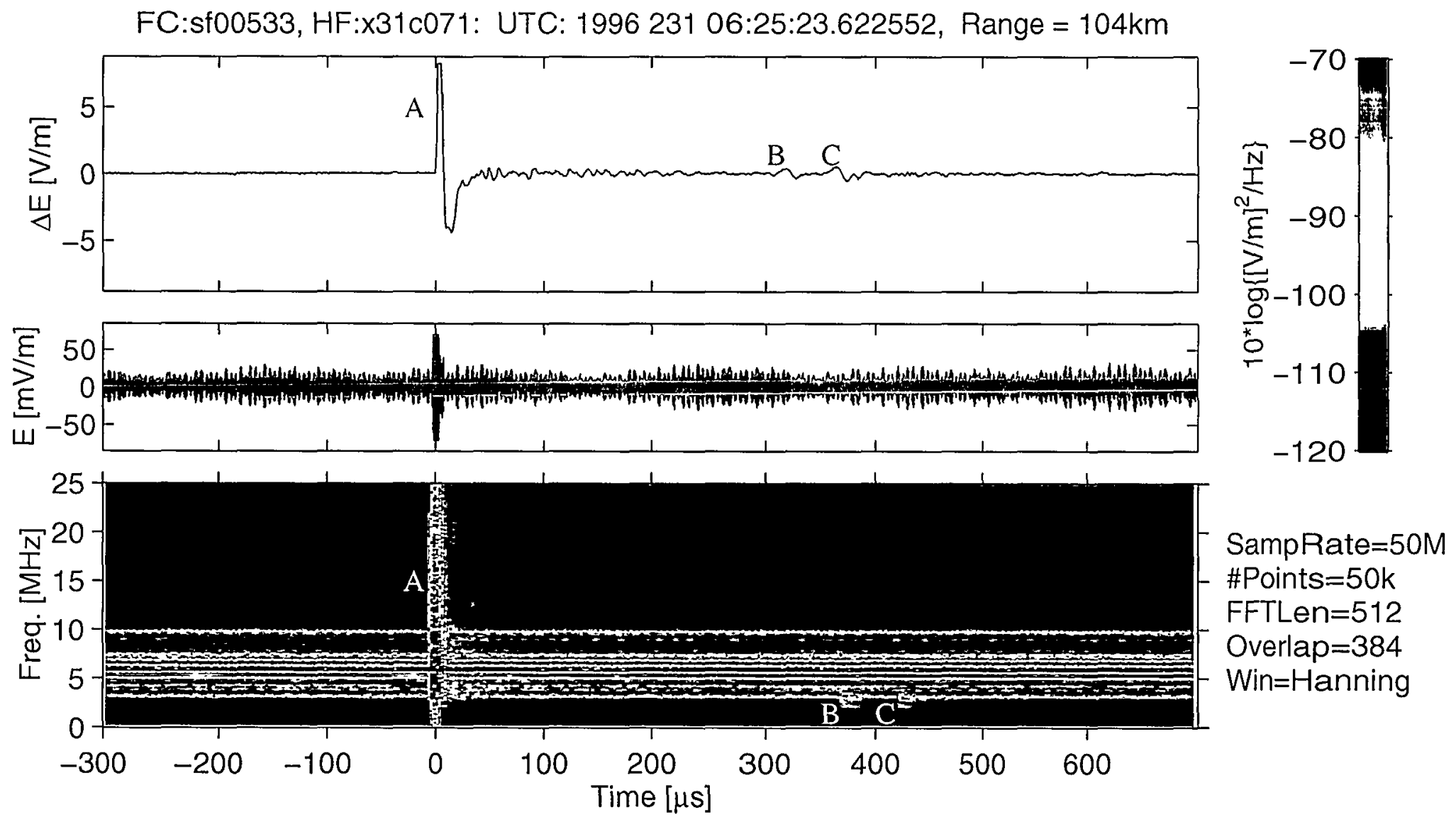

Figure 4.2: Electric field change waveform, broadband HF waveform, and HF spectrogram of a CID that occurred during storm 3. Both waveforms were saturated. Ionospheric reflections can be seen in the upper and lower panels and are labeled B and $\mathrm{C}$. 
distance from the source to the receivers was $104 \mathrm{~km}$ and the height of the source was $10.4 \mathrm{~km}$ MSL.

As stated in Chapter 3, reflections of both field change and HF signals from the ionosphere were often recorded. Both types of reflections are clearly visible in Figure 4.2. In the top panel the large bipolar pulse (labeled A) is the groundwave electric field change signal from the source. It propagated to the receiver along the surface of the earth. At delays of 312 and $361 \mu$ s are two smaller-amplitude pulses (labeled B and $\mathrm{C})$. The pulses are skywave signals that propagated from the source to the receiver via reflections from the earth and ionosphere. The first of the two pulses is the direct reflection of the source from the ionosphere (in this case, the E-layer). The second is the 1-hop ionospheric reflection of the ground reflection of the source. The two reflected electric field change pulses are also barely discernible in Figure 4.1. They are labeled in the same manner as the pulses in Figure 4.2.

For an illustration of the three propagation paths (the groundwave path and two skywave paths), refer to Figure 3.2. In Figure 3.2 the paths were labeled with letters (A, B, and C) corresponding to the waveform features labeled in Figures 4.1 and 4.2. In some instances, multiple pulse pairs (as many as 6) were recorded in ordered sequences following the arrival of groundwave signals. This was the case for the NPBP electric field change waveform shown in Figure 4.3, which features three pulse pairs in sequence after the initial groundwave pulse. Multiple observed pairs correspond to multiple-hop ionospheric propagation paths from the source to the receiver. The timing of the reflections relative to each other and to the groundwave signal made it possible to accurately determine source heights and virtual ionosphere 


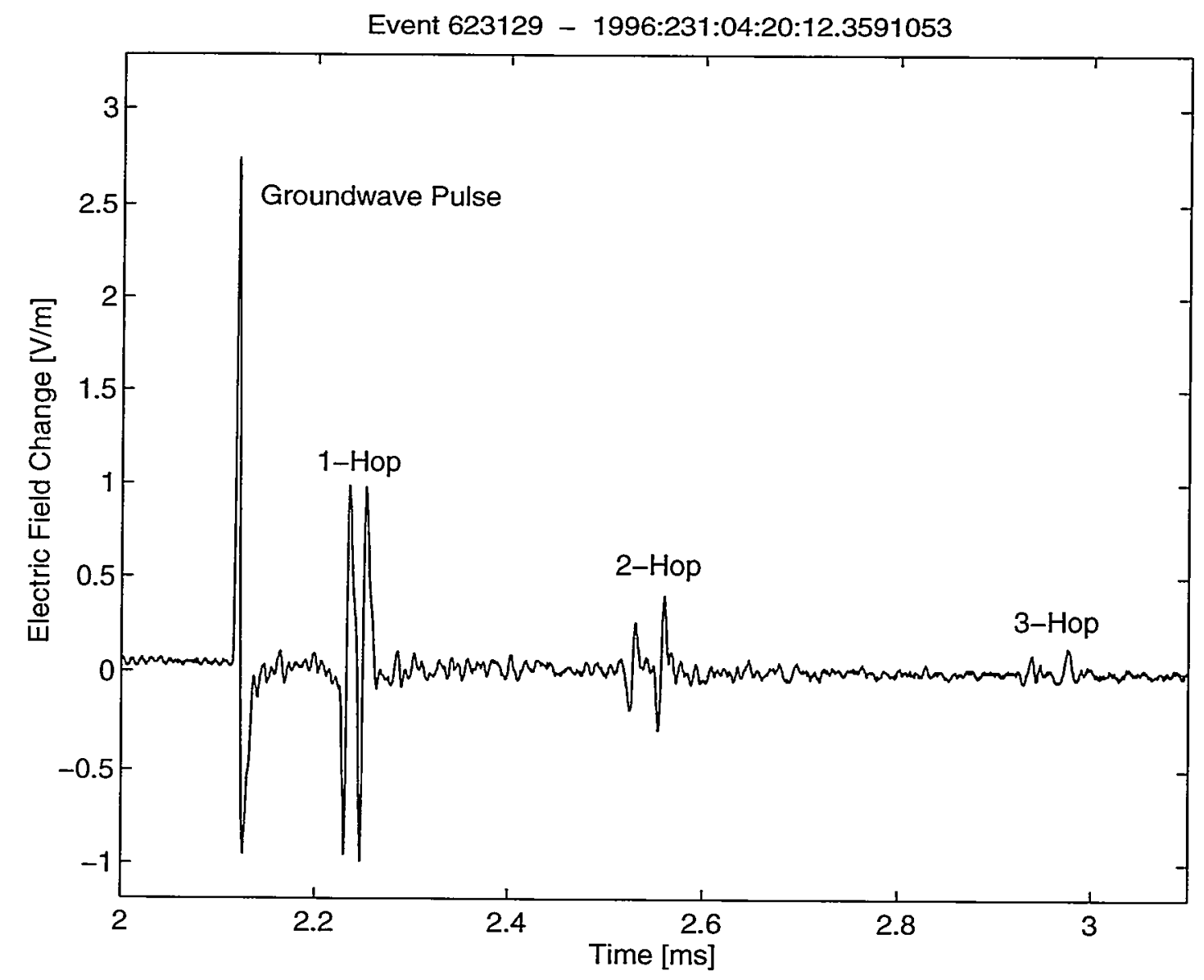

Figure 4.3: NPBP for which the groundwave and multiple-hop ionospheric reflections were recorded. 
heights using differential times of arrival. The event shown in Figure 4.3 was recorded on the same evening as the events recorded from storm 3, but was emitted from a thunderstorm in Mexico. The event was recorded by the Socorro field change meter (FC3) from a distance of $428 \mathrm{~km}$. The height of the source was $7.1 \mathrm{~km}$ AGL. The methods used to locate the source were similar to those described in the previous chapter.

HF reflections are also clearly visible in Figure 4.2. The broadband HF transient (labeled A) in the lower panel of the figure is the line-of-sight HF signal from the source. It arrived at approximately the same time as the groundwave field change pulse shown in the top panel. The two subsequent HF signals (labeled $B$ and $C$ ), which are visible only at low frequencies (between 2 and $4 \mathrm{MHz}$ ) and occur at delays of 381 and $431 \mu \mathrm{s}$, are reflections of $\mathrm{HF}$ emissions from the ionosphere and earth (again refer to Figure 3.2 for an illustration of the propagation paths). Note that the HF reflections arrived approximately $70 \mu$ s later than the field change reflections. This observation is consistent with the virtual ionospheric reflection height for the low frequency field change signals being lower in altitude than that for the high frequency signals. This is an expected result for low-angle skywave propagation paths [Davies, 1990]. The lower frequency (VLF/LF) electric field change signals refract more easily and thus, turn over at lower electron densities. As shown later in this chapter, the virtual heights for the field change and HF signals were approximately 90 and $100 \mathrm{~km}$ respectively. Bandlimiting of the $\mathrm{HF}$ reflections is also an expected result of interaction with the ionosphere. The spectrogram shows that the maximum usable frequency (MUF) for the propagation path from the source to the 
receiver was approximately $4 \mathrm{MHz}$ at the time of the event. $\mathrm{HF}$ reflections are barely discernible in Figure 4.1 at the same delays as in Figure 4.2. A less powerful source in combination with changing ionospheric conditions is sufficient to explain the weaker HF reflections in Figure 4.1.

NPBPs recorded from storms 1 and 2 were similar to those recorded from storm 3 , with a few noteworthy exceptions. Figure 4.4 shows an event recorded from storm 1. The range to the source was $185 \mathrm{~km}$. The height of the source was $8.2 \mathrm{~km}$ MSL. In most respects the event is very much like those pictured in Figures 4.1 and 4.2. Two weak electric field change reflections are discernible at delays of 247 and $278 \mu$ s in the top panel. However, no HF reflections are visible in the spectrogram. This was the case for all NPBPs observed during storm 1. This fact suggests that the ionospheric conditions were not suitable for the reception of $\mathrm{HF}$ reflections from the thunderstorm; i.e. the receiver was within the skip zone of the source. This was not unusual for HF propagation over distance of less than $200 \mathrm{~km}$ during the summer of 1996 and was actually more often the case than not. Note that the summer of 1996 coincided with a minimum in the solar cycle. The sensing geometry for storm 1 is illustrated in the upper panel of Figure 4.5. The illustration shows that no HF signals were reflected to the receivers. The three panels of Figure 4.5 show the sensing geometries for storms 1 through 3 respectively.

Figure 4.6 shows an event recorded from storm 2 . The distance to the event was $453 \mathrm{~km}$, much further than the distances to events recorded from storms 1 and 3 . The height of the source was $9.9 \mathrm{~km}$ MSL. As a consequence of the long range to the source, the delays from the time of arrival of the electric field change groundwave 


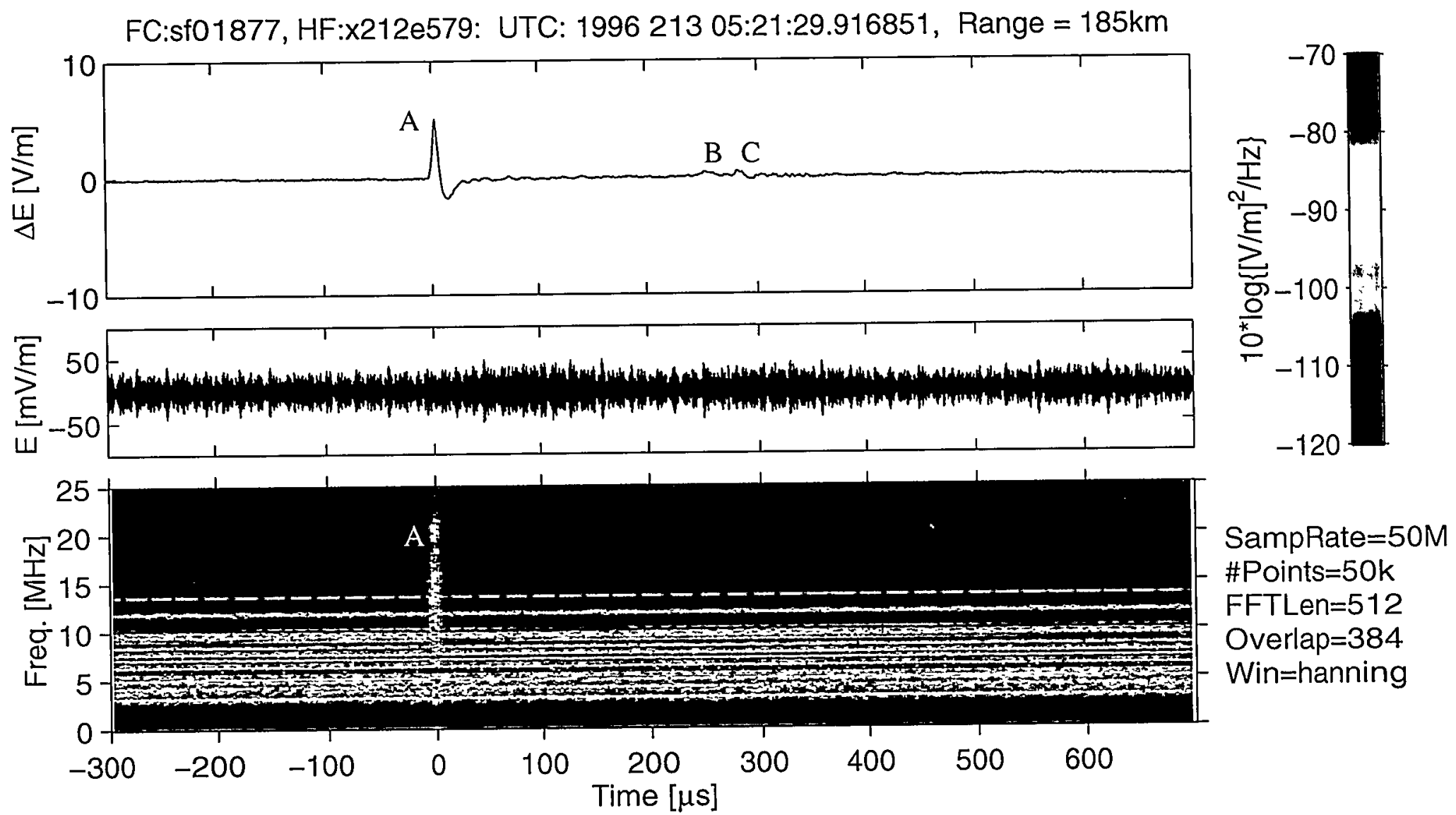

Figure 4.4: Electric field change waveform, broadband HF waveform, and HF spectrogram of a CID that occurred during storm 1. Two ionospheric electric field change reflections can be seen in the upper panel and are labeled B and C. No HF reflections were recorded during storm 1. 


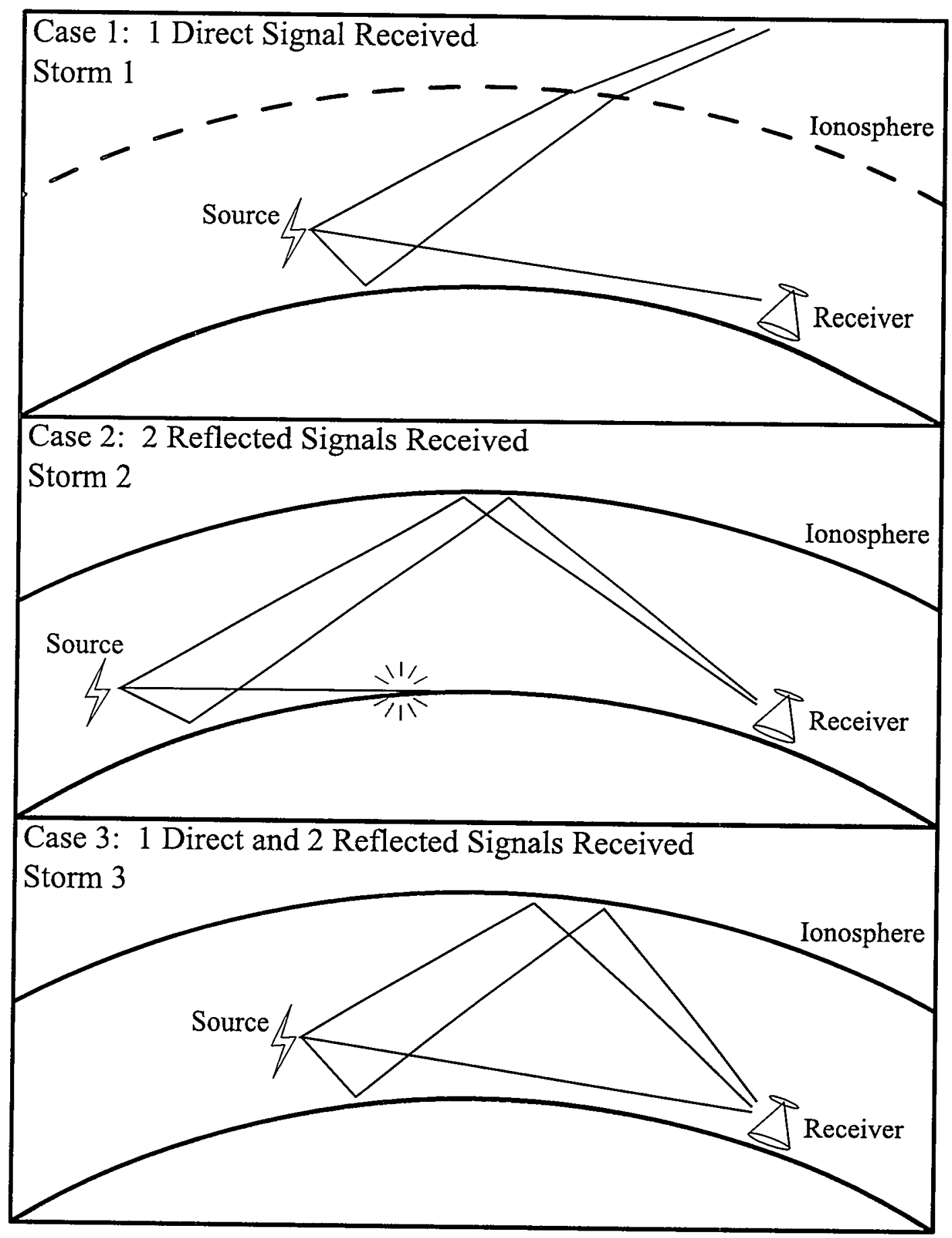

Figure 4.5: Illustrations of the $\mathrm{HF}$ propagation paths for storms 1, 2, and 3 . During storm 1, only line-of-sight signals were received. During storm 2, only 1-hop reflections from the E-layer were received. During storm 3, both line-of-sight signals and E-layer reflections were received. 
FC:su00372, HF:x19e454: UTC: 1996220 06:44:27.409872, Range $=453 \mathrm{~km}$
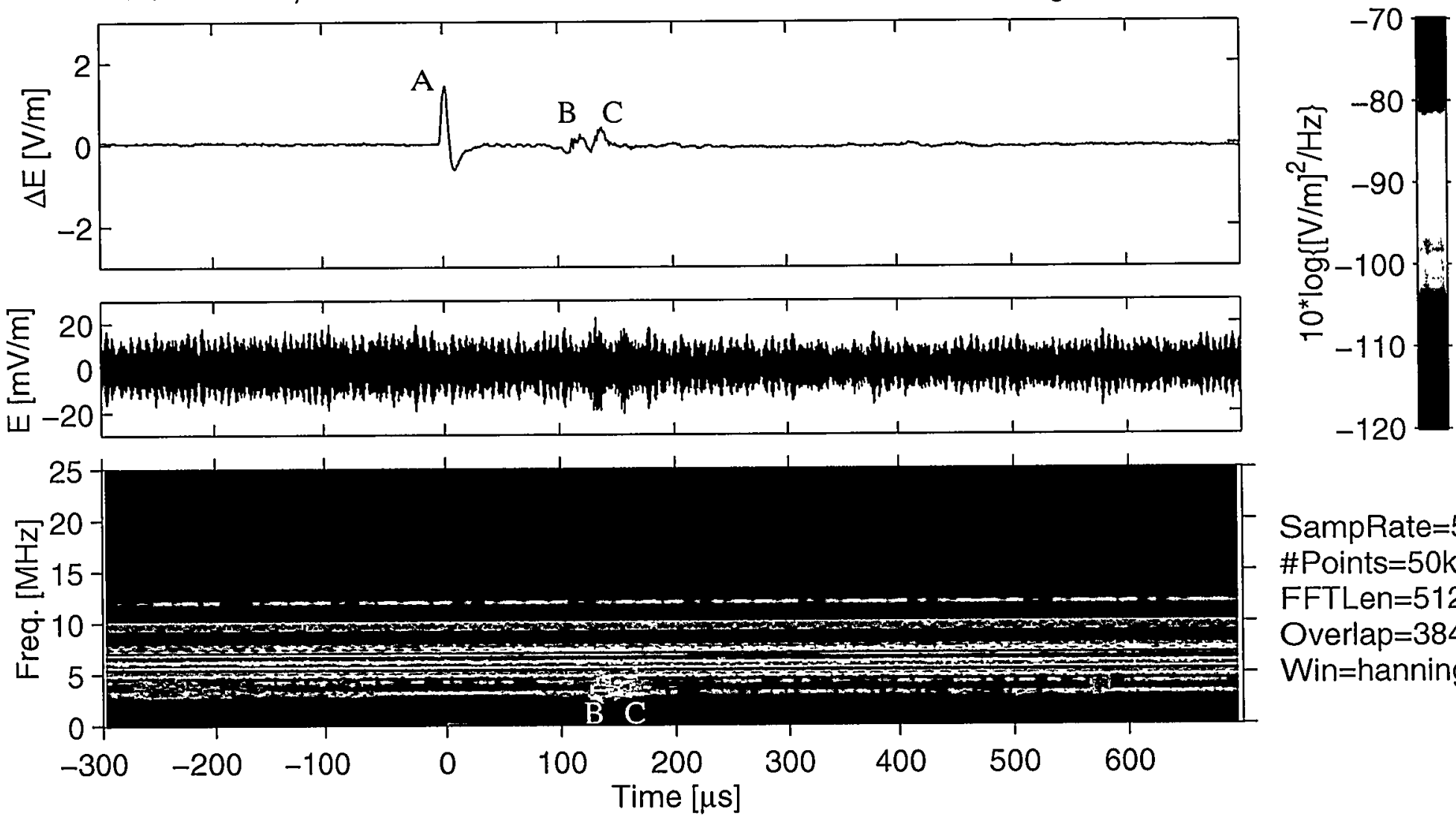

SampRate $=50 \mathrm{M}$

\#Points $=50 \mathrm{k}$

FFTLen $=512$

Overlap $=384$

Win=hanning

Figure 4.6: Electric field change waveform, broadband HF waveform, and HF spectrogram of a CID that occurred during storm 2. Ionospheric reflections can be seen in the upper and lower panels and are labeled B and C. No HF line-of-sight signals were recorded from storm 2 because the events occurred over the horizon. 
pulse to those of the two reflected pulses are shorter: 106 and 128 microseconds. The shorter delays result from the fact that the difference in the propagation path length between the groundwave path and a reflected path becomes smaller for propagation over further distances, since the reflected path meets the earth and ionosphere at increasingly oblique angles of incidence. Notice that no HF signal was received at the time of arrival of the field change groundwave pulse, but that two reflections are clearly visible in the HF record at delays of 135 and $157 \mu$ s. As explained earlier, the delays are greater than for the field change reflections because the signals reflected from greater ionospheric virtual heights. The distance to the source and the source height for this event were such that the event occurred beyond the radio horizon of the HF ground station; that is, the line-of-sight HF signal was blocked by the earth. The observation is consistent with a calculated distance of $391 \mathrm{~km}$ to the radio horizon for a source at $9 \mathrm{~km}$ and a receiver located at ground level (from Goodman [1992]). This case is illustrated in the center panel of Figure 4.5.

Figures $4.1,4.2,4.4$, and 4.6 show that depending on the distance to the source, the height of the source, and the ionospheric conditions, the recorded electric field change and HF emissions can appear quite different.

\subsection{WAVHFORMS FROM CI.OUD-TO-GROUND AND INTRACLOUD LIGHTNING}

To illustrate how distinct CID waveforms are in comparison to other thunderstorm radio emissions, it is useful to contrast the electric field change and HF 
waveforms shown in Figures 4.1, 4.2, 4.3, 4.4, and 4.6 with those produced by lightning processes more typical of those observed from thunderstorms. The comparison is most appropriate for the events pictured in Figures 4.1 and 4.2 because the lightning waveforms presented in this section were recorded from the same storm (and thus, from approximately the same distance) and were plotted using the same amplitude and power scales. Figure 4.7 shows the radio emissions from a negative cloud-to-ground lightning stroke that was recorded from storm 3 by the LANL field change and HF systems. The $1 \mathrm{~ms}$ record shows weak radiation from the last few hundred microseconds of a stepped leader followed by a negative-polarity return stroke. The weak stepped leader field change pulses in the top panel are separated by tens of microseconds and have polarities that are the same as that of the return stroke. These observations are consistent with those of Krider and Radda [1975], Krider et al. [1977] and Beasley et al. [1983]. The large negative-polarity feature that begins at time $-80 \mu \mathrm{s}$ is the signal from the actual return stroke. The polarity is indicative of the lowering of negative charge from overhead. Characteristics of the return stroke waveform are consistent with those presented by Weidman and Krider [1978]. A reflection of the return stroke signal from the ionosphere occurs at time $+260 \mu \mathrm{s}$.

The HF spectrogram in the lower panel of Figure 4.7 shows weak stepped leader RF pulses that occur prior to the return stroke. The leader inter-pulse periods are on the order of tens of microseconds. The leader RF characteristics are consistent with observations by Brook and Kitagawa [1964]. The strong radiation that occurs during the actual return stroke indicates that the stroke is the first stroke to ground of the 


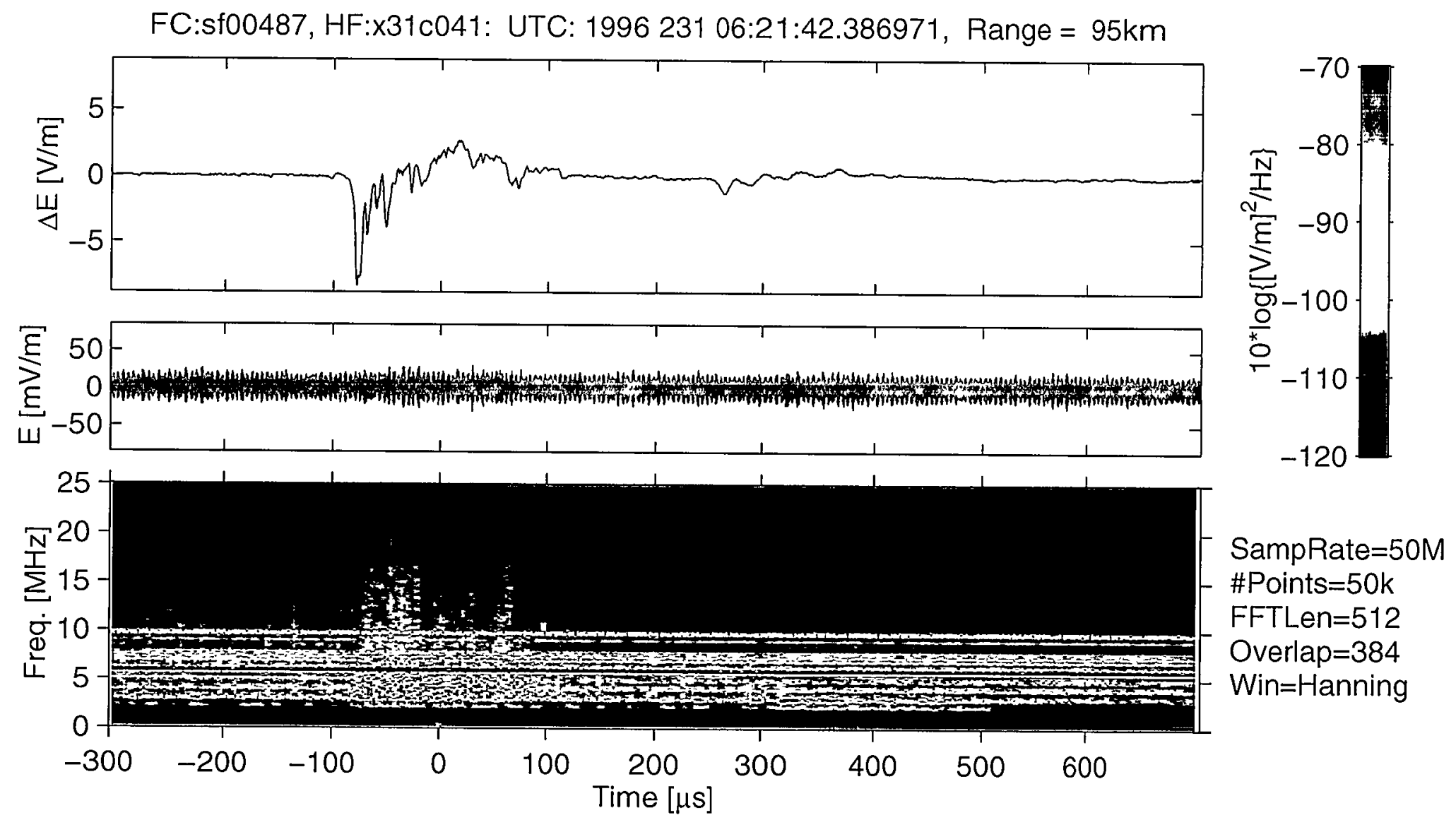

Figure 4.7: Electric field change waveform, broadband HF waveform, and HF spectrogram of a cloud-to-ground lightning stroke that occurred during storm 3 . 
flash. Subsequent strokes usually produce no or weak RF radiation [Brook and Kitagawa, 1964; Willett et al., 1990; Rhodes et al., 1994; Shao et al., 1995].

Figure 4.8 shows the radio emissions recorded during $1 \mathrm{~ms}$ of an intracloud flash from storm 3. The electric field change record shows a number of small-amplitude, initially-positive, bipolar pulses separated by time intervals ranging from tens to hundreds of microseconds. The HF spectrogram shows radio bursts with similar inter-pulse periods that often occur in coincidence with the electric field change features. The field change and RF observations of the intracloud flash are consistent with observations of intracloud flashes by Kitagawa and Brook [1960], Krider et al. [1979], and Weidman and Krider [1979].

The CIDs described in this chapter, as well as numerous others recorded during the summer of 1996, share a number of characteristics that clearly distinguish them from cloud-to-ground and intracloud flashes like those pictured in Figures 4.7 and 4.8. Among the defining characteristics of the events are their usual occurrence in temporal isolation from other events, their short durations, and their large peak amplitudes. These defining characteristics will be quantified in Chapter 6 .

\subsection{CID LOCATIONS}

CID sources were located within the three storms using the differential time of arrival techniques described in Chapter 3. The following sections summarize the results obtained from each of the storms. 


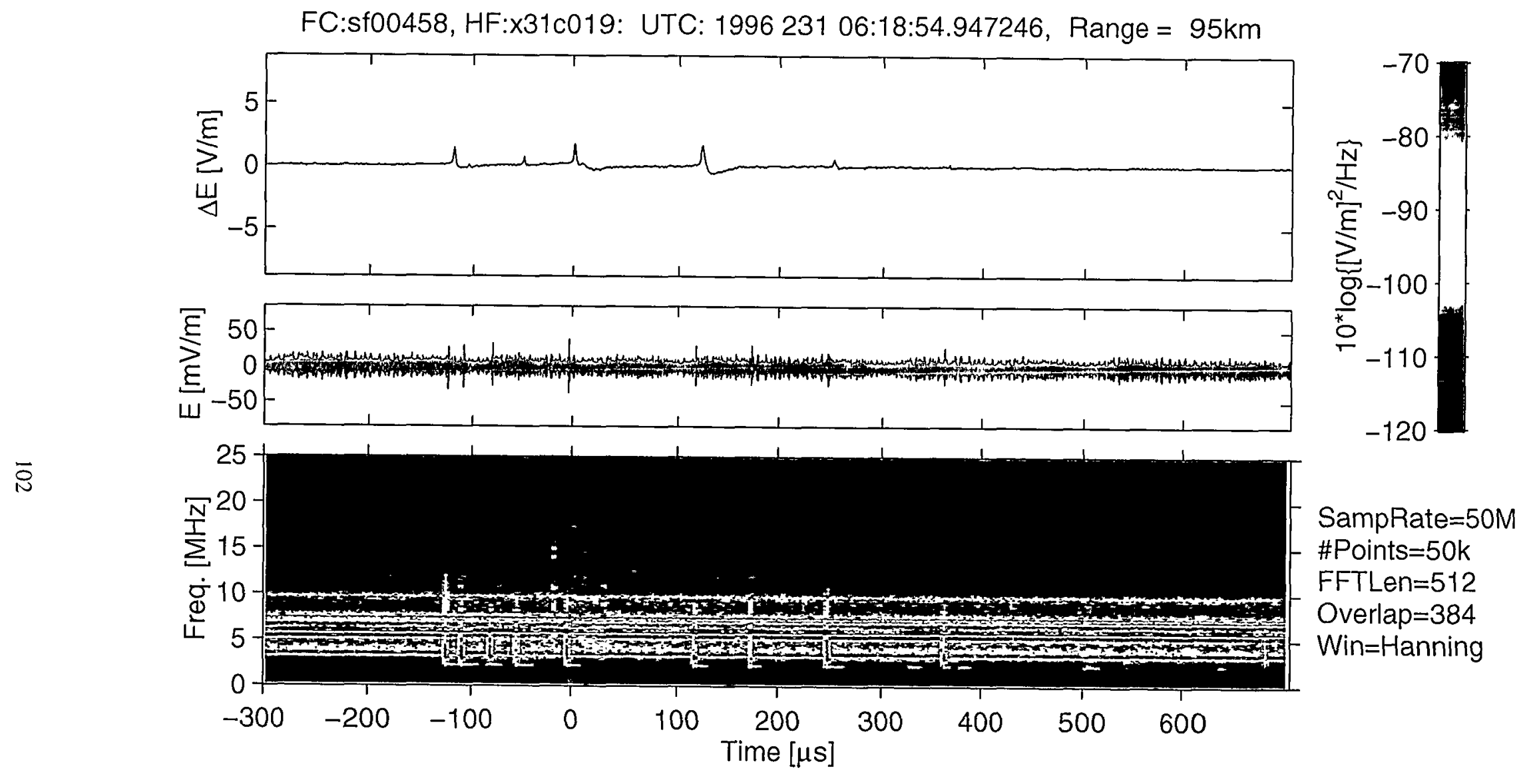

Figure 4.8: Electric field change waveform, broadband $\mathrm{HF}$ waveform, and $\mathrm{HF}$ spectrogram of a portion of an intracloud lightning flash that occurred during storm 3 . 


\subsubsection{Storm 1}

The locations of CIDs recorded during storm 1 are shown in plan view in Figures 2.1 and 4.9, where they are represented by diamonds. Five NPBPs were recorded by stations FC1, FC2, HF1, HF2, and HF3 between 23:06 and 23:25 MDT (local time) on 30 July 1996. The numbers next to the diamonds in Figure 4.9 indicate the time order of occurrence of the five events. The CIDs were located using location method one, which was described in Section 3.1.1. The figure also shows the locations of cloud-to-ground lightning flashes reported by the National Lightning Detection Network (NLDN) during the same time period. Negative CGs are represented as dots in the figure and positive CGs are represented as plus signs. The size and shape of the $\mathrm{CID}$ location error uncertainty ellipse is shown in the legend. The ellipse was computed based on the uncertainties in the timing of the groundwave field change and line-of-sight HF signals from the events. The ellipse is highly eccentric with its major axis aligned with the vector to the recording stations (toward the southwest). Four of the five CIDs occurred along a line parallel to the major axis of the error ellipse. The size and shape of the ellipse easily allows for the possibility that the four CIDs occurred in the same region of the storm that produced the majority of the cloud-toground lightning activity. The computed location of the fifth $\mathrm{CID}$ was $15 \mathrm{~km}$ southeast of the line that contained the other $4 \mathrm{CDs}$. Unless a gross timing error occurred ( $>20 \mu \mathrm{s}$ ), which was much greater than the assumed timing uncertainties (2 to $5 \mu \mathrm{s}$ ), the fifth $\mathrm{CID}$ appears to have occurred in a different location from the 


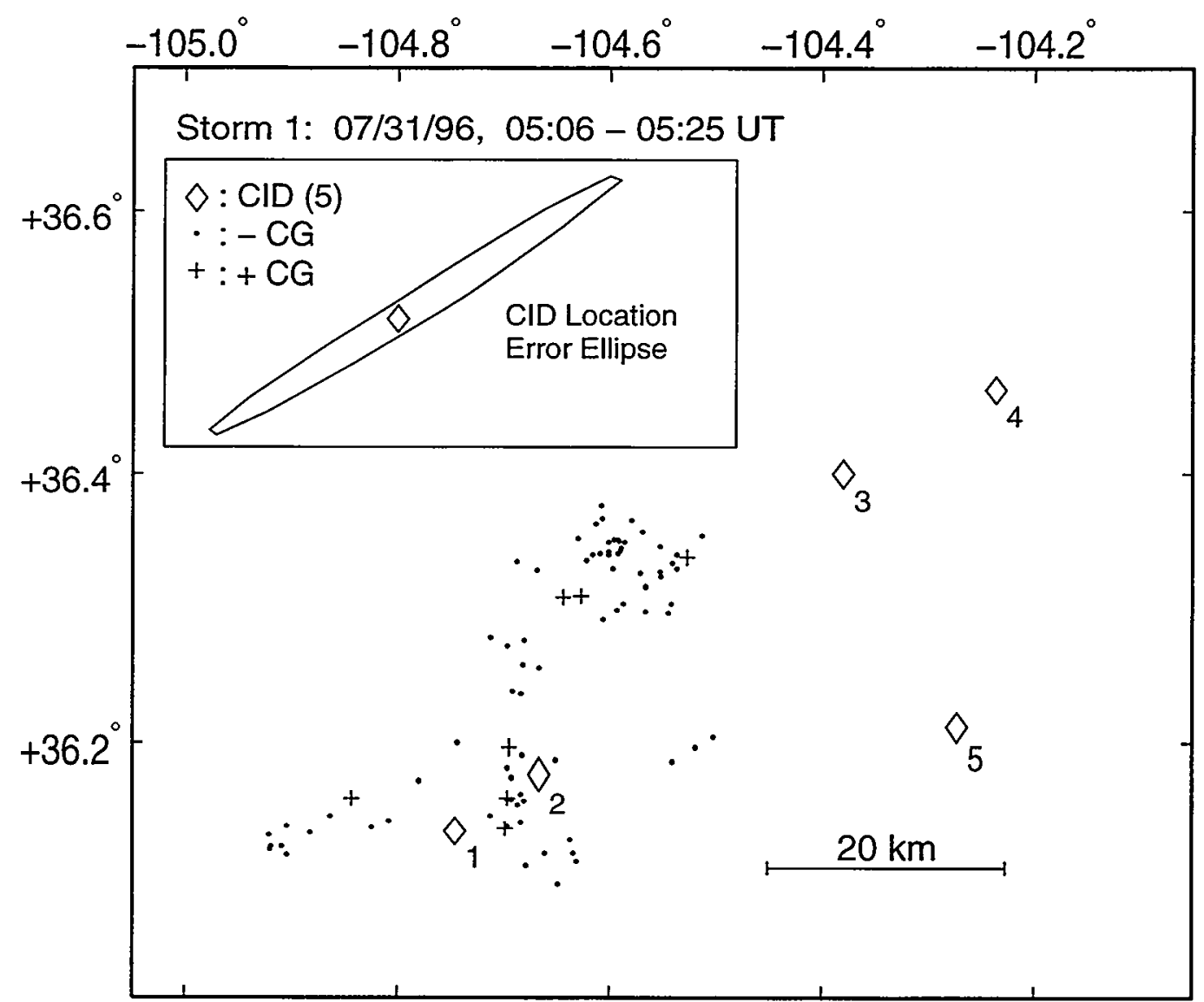

Figure 4.9: Locations of events recorded during storm 1. CID locations are represented by diamonds. Negative CG locations (as reported by the NLDN) are represented by dots. Positive CG locations are represented by plus symbols. 
previous four. The location uncertainties for events reported by the NLDN have been claimed to be on the order of a few kilometers.

The heights of CIDs recorded from storm 1 as a function of the local time of day are shown in Figure 4.10. Heights were computed using ionospheric reflection delays from the $\mathrm{FCl}$ and $\mathrm{FC} 2$ field change stations (the $\mathrm{HF}$ stations did not receive reflections from the events recorded during this storm). $\mathrm{FCl}$ heights are represented as blue circles. FC2 heights are represented as red circles. Black plus signs indicate the mean values for heights recorded by the two stations. The plus signs are joined by a black line. There does not appear to be any trend in source altitude during the time the CIDs were recorded. The mean source height and standard deviation for CIDs recorded during storm 1 was $8.7 \pm 0.4 \mathrm{~km} \mathrm{MSL}$. The median source height was also 8.7 km MSL. The calculated measurement uncertainty was $0.6 \mathrm{~km}$.

\subsubsection{Storm 2}

The locations of CIDs recorded during storm 2 are shown in plan view in Figures 2.1 and 4.11. Eleven CIDs were recorded by stations FC1, FC2, and HF1 between 00:26 and 00:56 MDT on 7 August 1996. Numbers next to the diamonds in Figure 4.11 indicate the time order of occurrence of the eleven events. The CIDs were located using location method two, which was described in Section 3.1.2. The figure also shows the locations of cloud-to-ground lightning flashes reported by the NLDN during the same time period. The error ellipse for the 2-D CID locations is shown in 


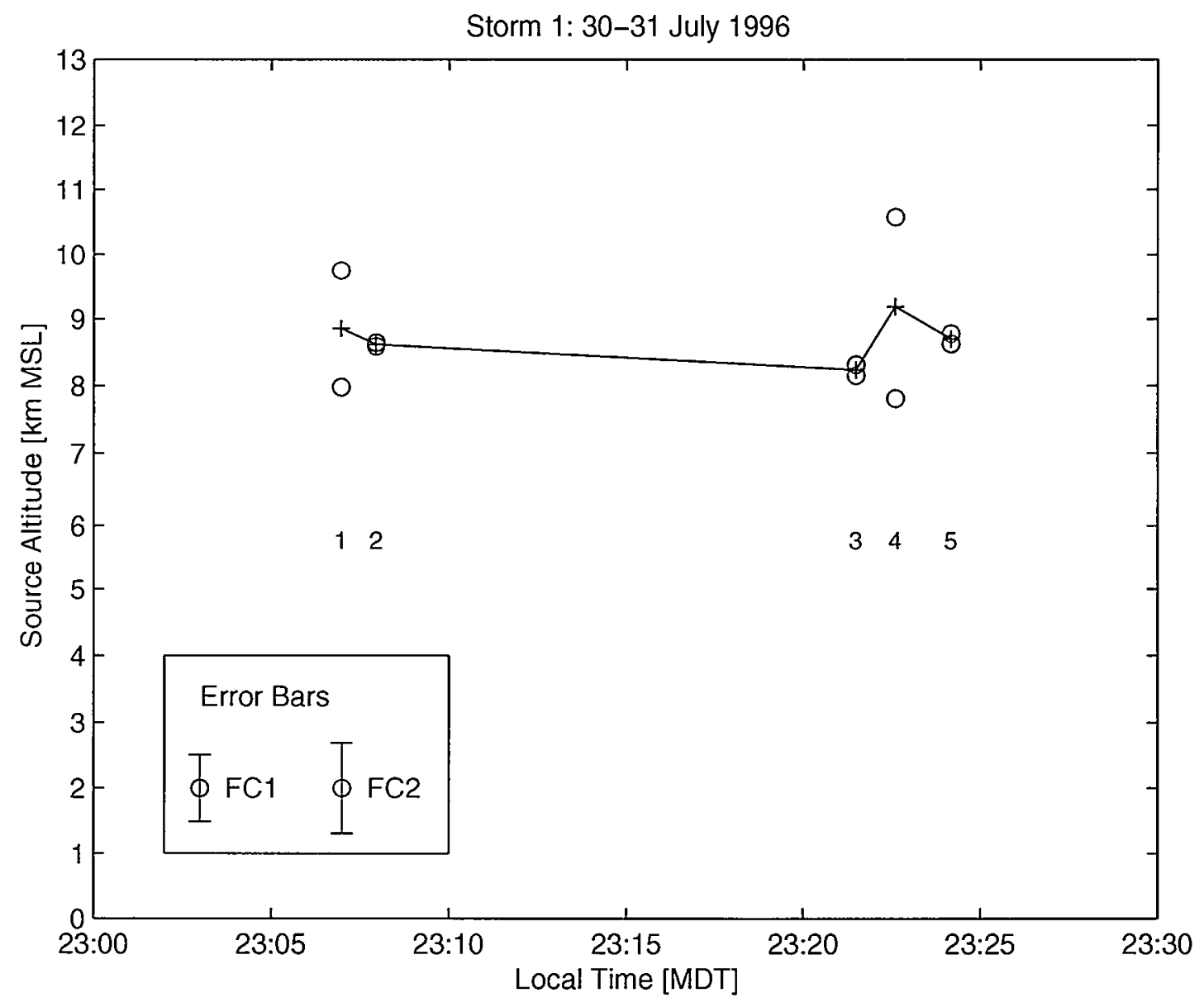

Figure 4.10: Time-altitude plot for CIDs recorded during storm 1. Circles show the altitudes determined from individual recording stations. Black plus signs show the mean altitudes determined for each event. 


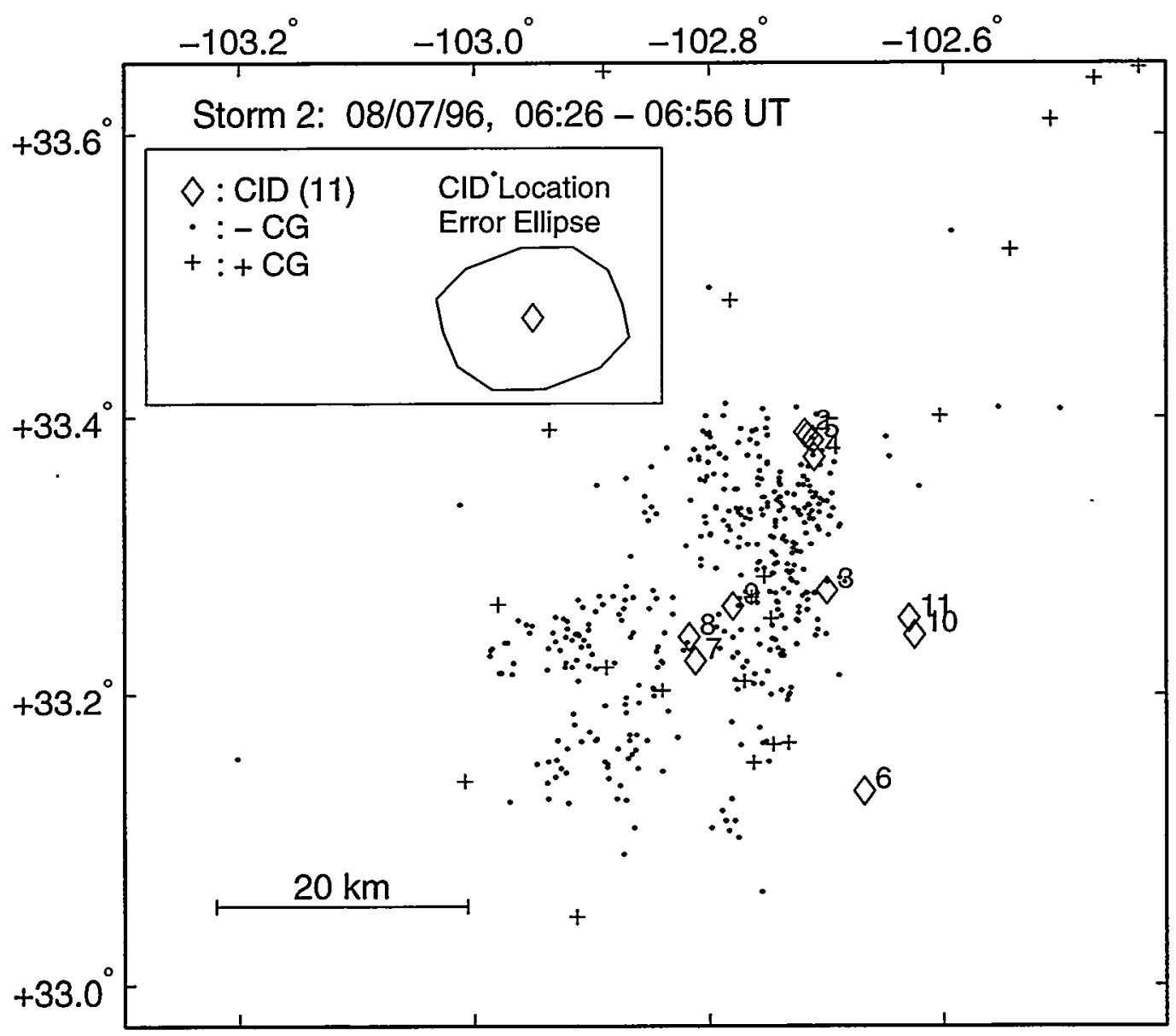

Figure 4.11: Locations of events recorded during storm 2. CD locations are represented by diamonds. Negative CG locations (as reported by the NLDN) are represented by dots. Positive CG locations are represented by plus symbols. 
the legend. All of the events occurred in the same region of the storm that produced the cloud-to-ground lightning activity.

The heights of CDs recorded from storm 2 as a function of the local time of day are shown in Figure 4.12. Heights were computed using reflection delays from the $\mathrm{FCl}, \mathrm{FC} 2$, and $\mathrm{HF} 1$ recording stations and are indicated by blue, red, and green circles respectively. Black plus signs indicate the mean height values for each event. The mean values are joined by a black line. There is some suggestion that the altitude at which the CIDs occurred lowered by as much as a kilometer during the time period when the discharges were recorded. The mean source height and standard deviation for CIDs recorded during storm 2 was $9.6 \pm 0.7 \mathrm{~km}$ MSL. The median source height was $9.5 \mathrm{~km}$ MSL. The calculated measurement uncertainty was $0.8 \mathrm{~km}$.

\subsubsection{Storm 3}

The locations of CIDs recorded during storm 3 are shown in plan view in Figures 2.1 and 4.13. Eight $\mathrm{CIDs}$ were recorded by stations FC1, FC2, FC3, and HF1 between 23:43 and 00:29 MDT on 18 and 19 August 1996. Numbers next to the diamonds in Figure 4.13 indicate the time order of occurrence of the eight events. The CIDs were located using location method one from Section 3.1.1. The figure also shows the locations of cloud-to-ground lightning flashes reported by the NLDN during the same time period. The error ellipse for the 2-D CID locations is shown in the legend. The large eccentricity of the ellipse is due to the station locations with respect to the sources. The source location uncertainty for events recorded from 
Storm 2: 6-7 Aug. 1996

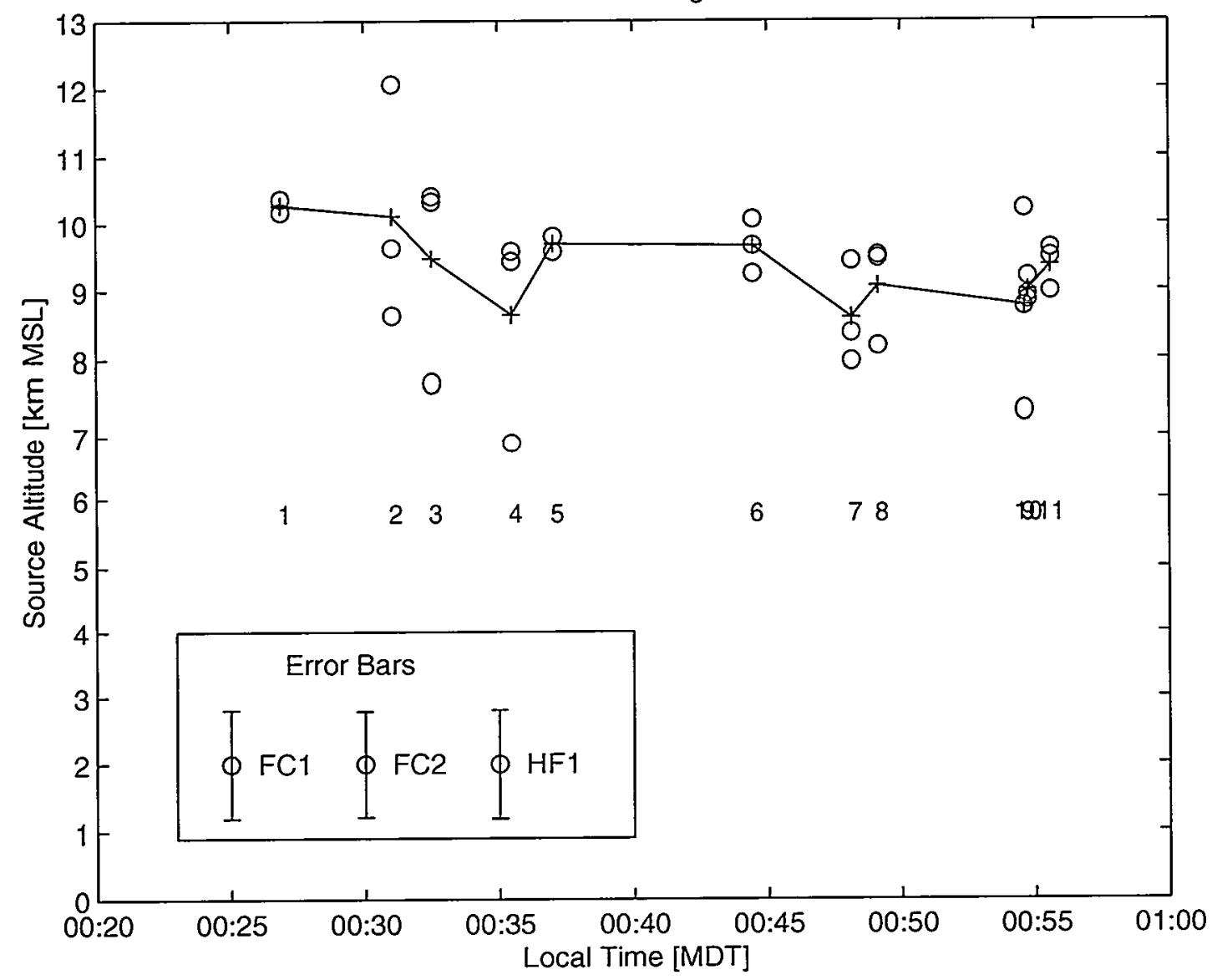

Figure 4.12: Time-altitude plot for CIDs recorded during storm 2. Circles show the altitudes determined from individual recording stations. Black plus signs show the mean altitudes determined for each event. 


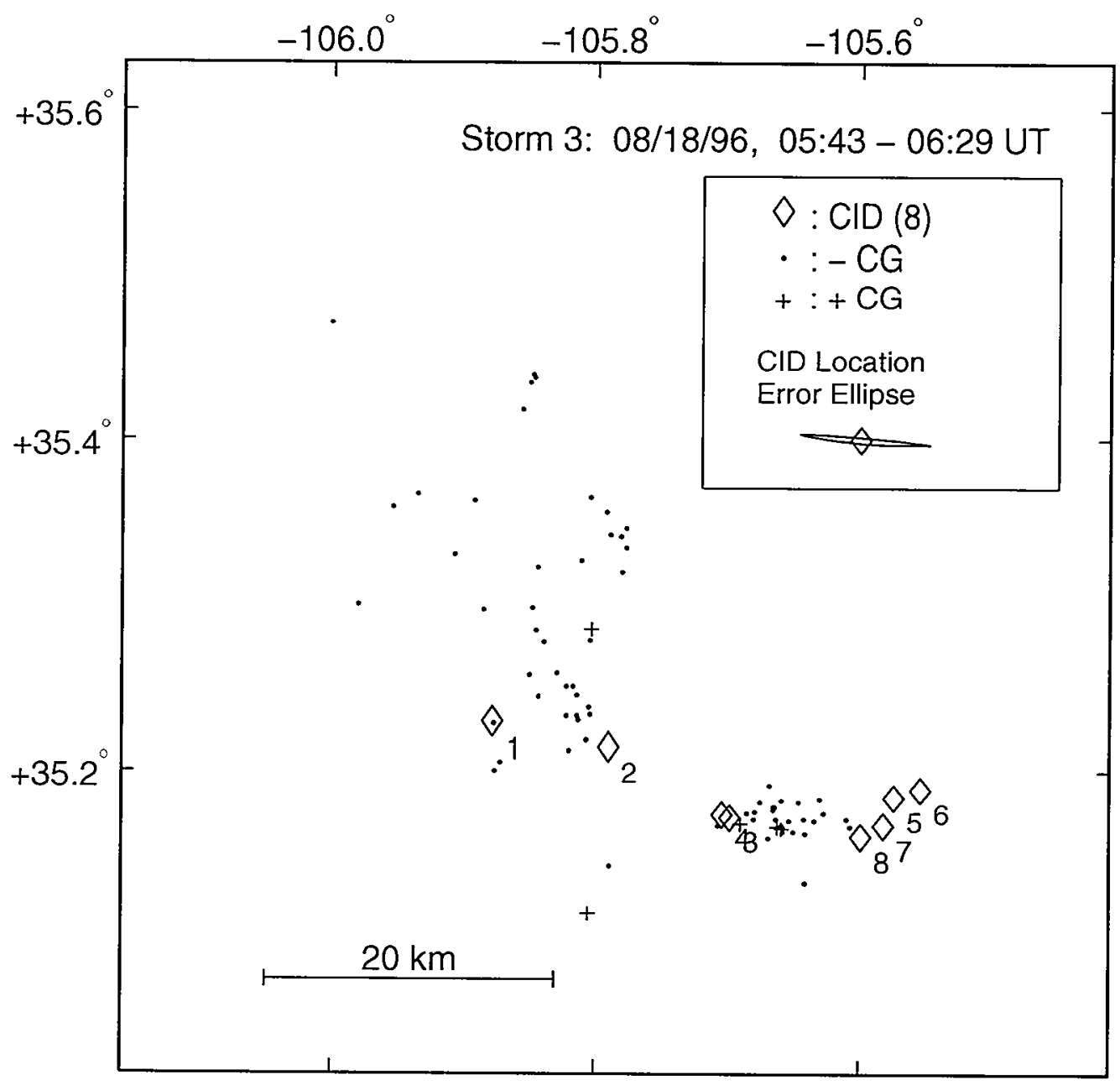

Figure 4.13: Locations of events recorded during storm 3. CID locations are represented by diamonds. Negative CG locations (as reported by the NLDN) are represented by dots. Positive CG locations are represented by plus symbols. 
storm 3 is smaller than those for storms 1 and 2, especially in latitude. It is approximately $0.4 \mathrm{~km} \times 8.0 \mathrm{~km}$. Figure 4.13 shows that the CID source regions was well localized and moving toward the southeast. The source region was embedded within a less well localized region that produced the cloud-to-ground lightning activity.

The heights of CIDs recorded from storm 3 as a function of the local time of day are shown in Figure 4.14. Heights were computed using reflection delays from the $\mathrm{FC} 1, \mathrm{FC} 2, \mathrm{FC} 3$, and $\mathrm{HF} 1$ recording stations and are indicated by blue, red, cyan, and green circles respectively. Black plus signs indicate the mean height values for each event. The mean values are joined by a black line. There does not appear to be a source altitude trend. The mean source height and standard deviation for CIDs recorded during storm 3 was $9.6 \pm 0.6 \mathrm{~km} \mathrm{MSL}$. The median source height was also $9.6 \mathrm{~km}$ MSL. The calculated measurement uncertainty was $0.4 \mathrm{~km}$.

\subsubsection{Observations from all Three Storms}

Reflections of signals from the ionosphere and earth were used to compute 3-D source locations for $24 \mathrm{CIDs}$ that occurred in three southwestern thunderstorms. As described in Chapter 3, each source height determination also required that the virtual height of the ionosphere be determined. For 12 of the events, ionospheric reflections of both electric field change and broadband $\mathrm{HF}$ emissions were recorded. For these events, independent source heights were computed using the two types of recorded emissions. For the remaining 12 events, only electric field change reflections were 
Storm 3: 17-18 Aug. 1996

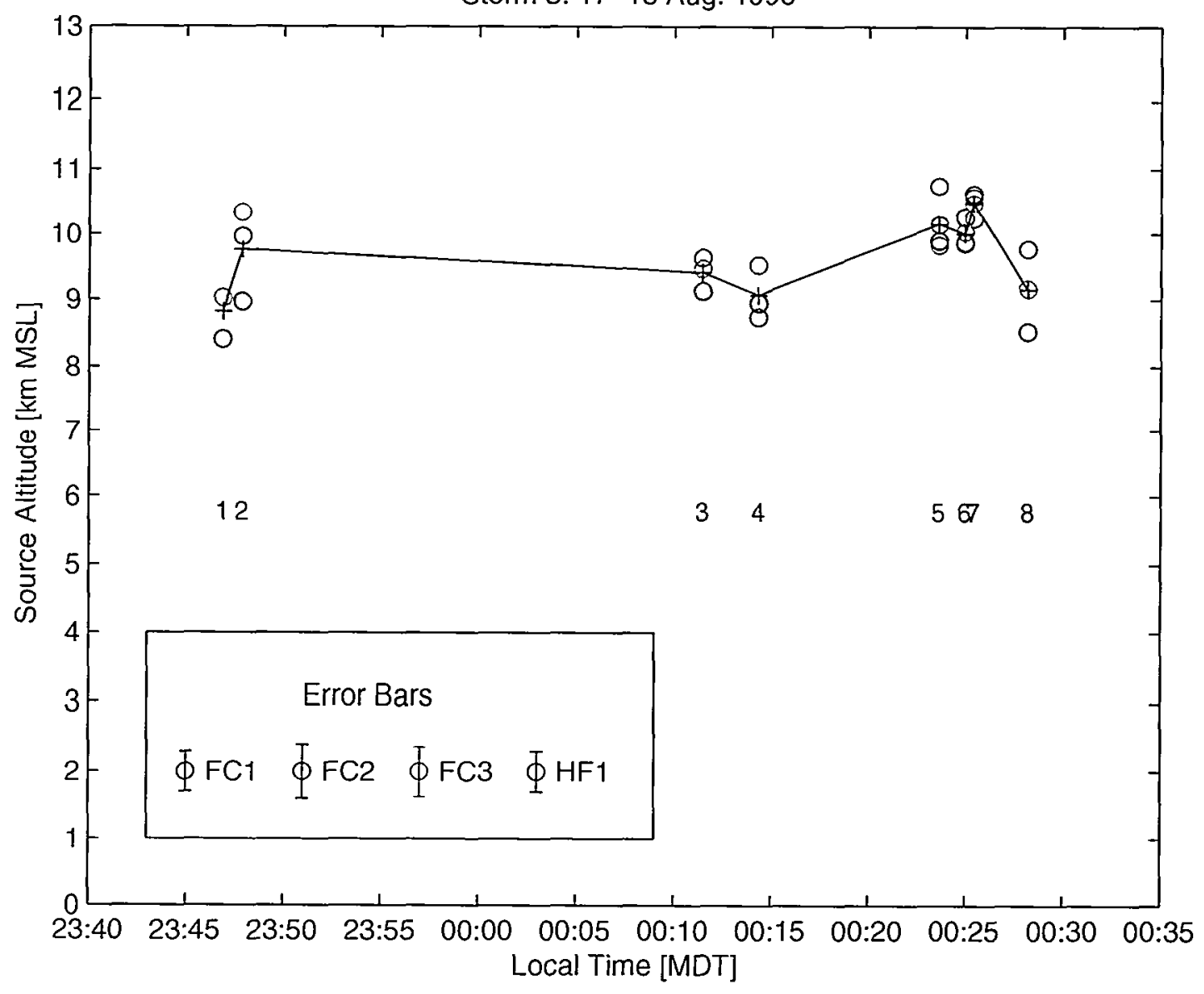

Figure 4.14: Time-altitude plot for CIDs recorded during storm 3. Circles show the altitudes determined from individual recording stations. Black plus signs show the mean altitudes determined for each event. 
received. The lack of $\mathrm{HF}$ reflections in each case was due either to the fact that the receivers were within the ionospheric skip zone of the source, or to the fact that the reflections were too weak to time tag accurately. For these events, source heights were computed using electric field change reflections only.

The mean source height for all 24 events, based on electric field change data only, was $9.43 \pm 0.68 \mathrm{~km}$ MSL. The median source height was $9.36 \mathrm{~km}$ MSL. The mean source height for 12 of the events, based on HF data only, was $8.92 \pm 1.15 \mathrm{~km}$ MSL. The median source height was $9.23 \mathrm{~km}$ MSL. The distributions of source heights for both the field change and HF calculations are shown in Figure 4.15. The distributions have mean and median values that agree to within about $0.5 \mathrm{~km}$. Recall that height uncertainties were on the order of 0.4 to $0.8 \mathrm{~km}$. The data show that that the sources of the electric field change emissions are co-located with the sources of the broadband HF emissions within the resolution of the measurements. Source heights for the individual storms (based on both FC and HF data) are summarized in Table 4.1.

An interesting result was obtained in converting CID altitudes above ground level to altitudes above mean sea level using the ground elevations of the source regions. In doing so, the distribution of source heights became narrower. This is apparent in comparing the standard deviation of the AGL height distributions to that of the MSL height distribution. These standard deviations were 0.84 and $0.68 \mathrm{~km}$ respectively. A possible interpretation of this result is that the thunderstorm conditions that are necessary to spawn CID events are more closely related to altitude MSL than altitude AGL. This result is consistent with observations by Paul Krehbiel (private 

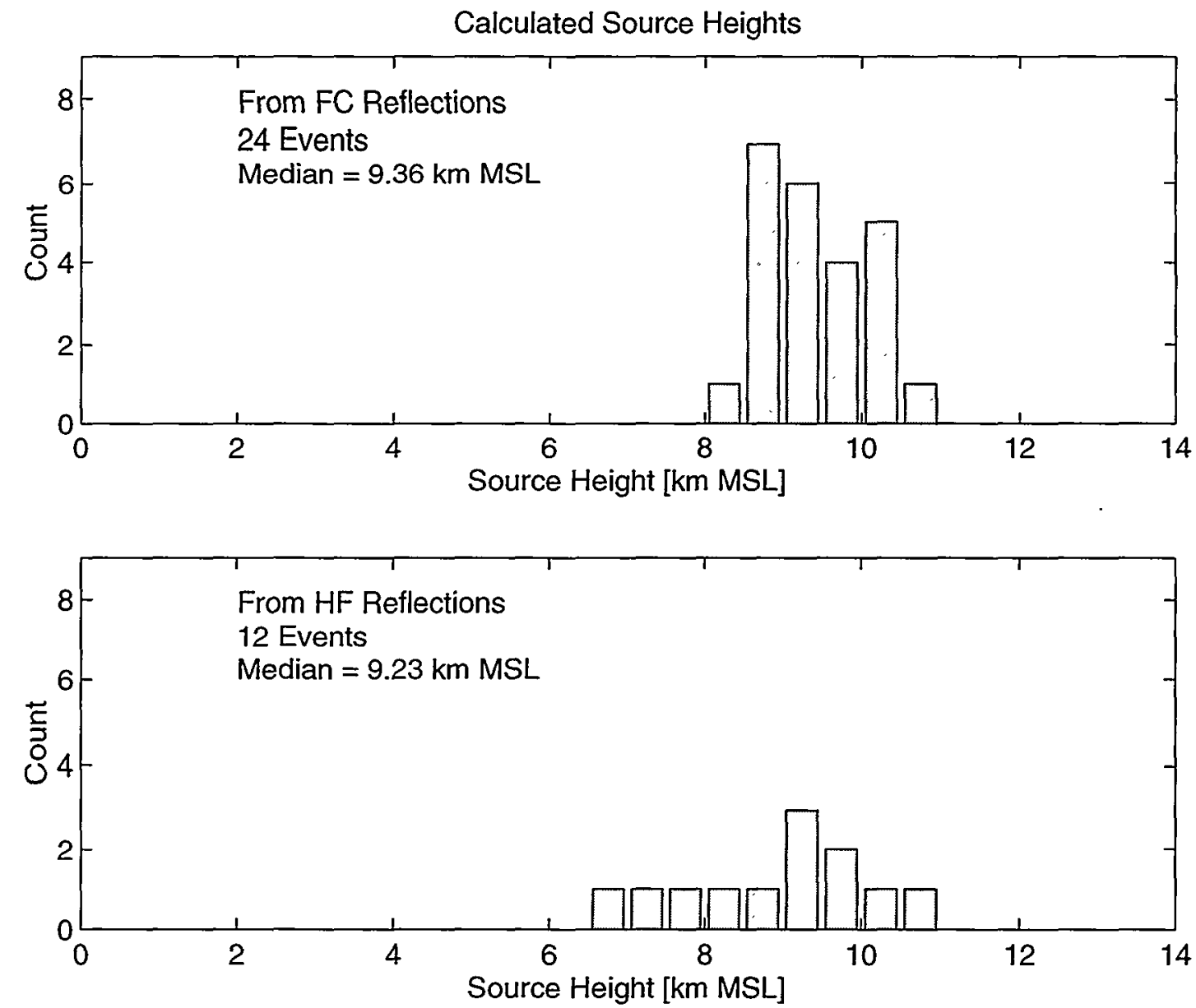

Figure 4.15: The upper panel shows the altitudes of $24 \mathrm{CDD}$ as determined from timing data from the electric field change stations. The lower panel shows the altitudes of $12 \mathrm{CIDs}$ as determined from timing data from the broadband HF stations. The histograms have similar median values. 
communication) that indicate that lightning phenomena tend to be associated with isotherms and that for a given type of thunderstorm the isotherms remain at fairly constant altitudes above mean sea level.

\begin{tabular}{|l|l|l|l|l|}
\hline & All Storms & Storm 1 & Storm 2 & Storm 3 \\
\hline Numbers of Events & 24 & 5 & 11 & 8 \\
Height of Ground [km MSL] & - & 1.8 & 1.2 & 2.1 \\
Mean Source Altitude [km MSL] & $9.4 \pm 0.7$ & $8.7 \pm 0.3$ & $9.6 \pm 0.7$ & $9.6 \pm 0.6$ \\
Source Altitude Range [km MSL] & $8.2-10.8$ & $8.2-9.2$ & $8.7-10.8$ & $8.8-10.4$ \\
Mean Ionosphere Altitude [km MSL] & $92.0 \pm 2.9$ & $96.7 \pm 3.1$ & $91.2 \pm 0.4$ & $90.0 \pm 0.5$ \\
Ionosphere Altitude Range [km MSL] & $90.8-100.0$ & $92.7-100.0$ & $90.8-92.2$ & $89.5-90.9$ \\
\hline
\end{tabular}

Table 4.1: Summary of Source and Ionosphere Height Observations.

The mean ionospheric virtual height calculated from electric field change data for the $24 \mathrm{CDs}$ was $92.0 \pm 2.9 \mathrm{~km}$ MSL. The median value was $91.0 \mathrm{~km}$ MSL. The mean ionospheric virtual height calculated from broadband HF data for 12 of the NPBPs was $101.9 \pm 0.7 \mathrm{~km}$ MSL. The median value was $101.6 \mathrm{~km}$ MSL. The distributions of ionospheric virtual heights (above mean sea level) for both the field change and HF calculations are shown in Figure 4.16. Ionosphere virtual height results for the individual storms are summarized in Table 4.1. The field change virtual height and $\mathrm{HF}$ virtual height distributions have mean and median values that differ significantly from each other. The difference in the height computations results from the fact that when reflections of both electric field change signals and broadband 
Calculated lonosphere Heights
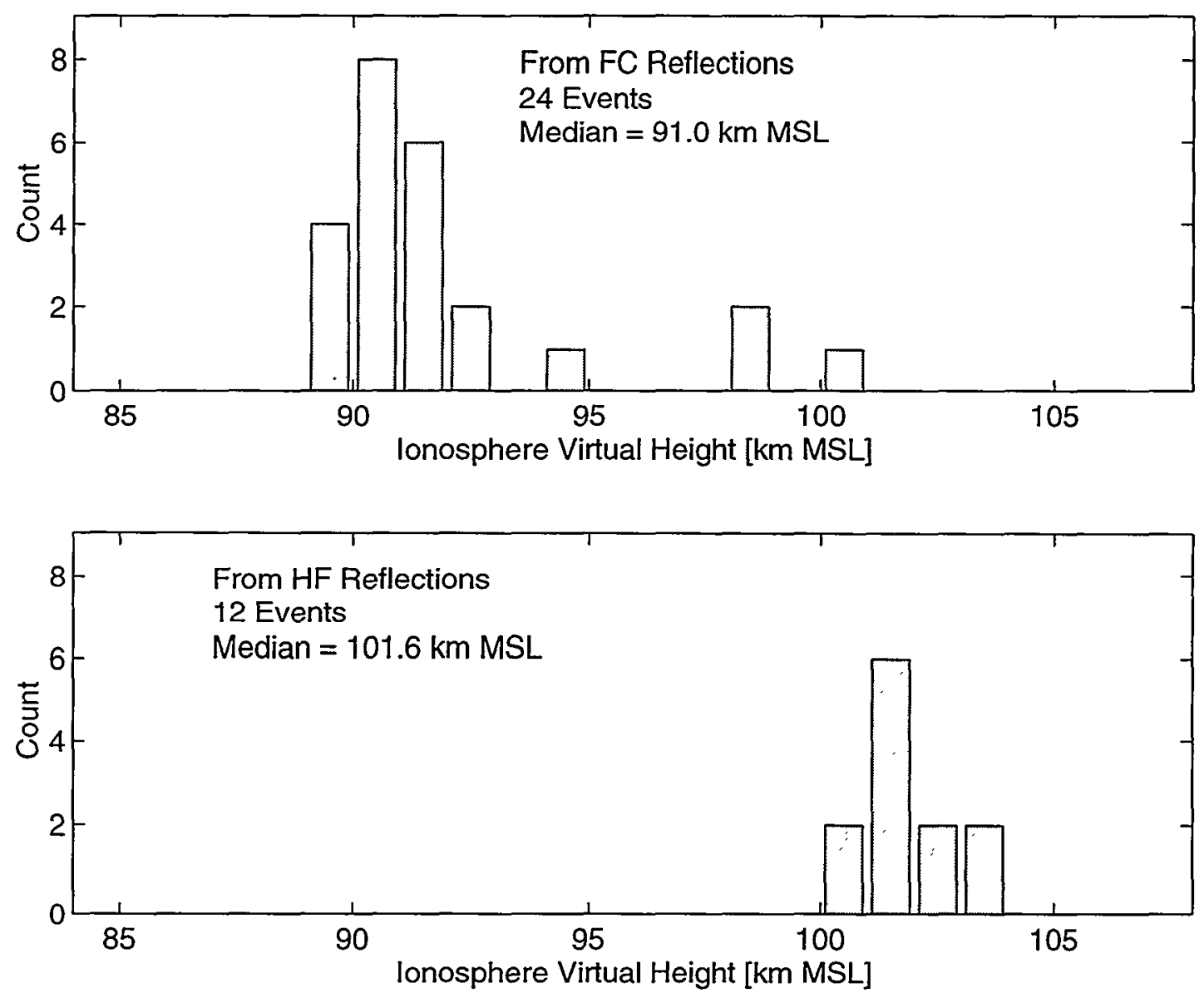

Figure 4.16: The upper panel shows ionosphere virtual heights as determined from $24 \mathrm{CIDs}$ recorded by the electric field change stations. The lower panel shows ionosphere virtual heights as determined from $12 \mathrm{CIDs}$ recorded by the broadband HF stations. The histograms have significantly different median values, a result consistent with the different frequency bands of the signals. 
HF signals were received, the field change signals arrived earlier, as if they had reflected from a lower altitude. This is, in fact, an expected result. The apparent discrepancy between the calculated ionospheric virtual height values can be attributed to the fact that ionospheric virtual heights for obliquely incident radio waves are a function of frequency with lower frequency waves being reflected from lower virtual heights. The median value of $91.0 \mathrm{~km}$ MSL for the field change emissions, which are VLF/LF radio waves, is consistent with results reported by Appleton and Barnett [1925] and Bracewell et al. [1951] for obliquely propagated VLF/LF waves over

paths of similar lengths during the night. The median value of $101.6 \mathrm{~km} \mathrm{MSL}$ for the broadband HF emissions is consistent with results from Martyn et al. [1934]. The fact that source height analyses performed using field change data and HF data independently of one another yielded similar source heights determinations, but different (yet expected) ionosphere heights provides validation for the methods of using times of arrival of earth and ionosphere reflections to determine source heights.

\subsection{TEMPoral CONTEXT}

A remarkable characteristic of CIDs recorded during the summer of 1996 was their occurrence as single, isolated events. In 17 out of 24 electric field change records from CIDs, the NPBP (with its ionospheric reflections) was the only transient emission within the record. Records were between 4.1 and $8.2 \mathrm{~ms}$ in duration. In all 24 recordings of $\mathrm{HF}$ emissions from CIDs, there were no other detectable transient 
emissions. HF records were $1 \mathrm{~ms}$ in duration. The NMIMT electric field change data acquisition system (FC2) was able to re-arm nearly instantly (within a few milliseconds) following the recording of an event, so was able to acquire data nearly continuously during bursts of lightning activity. Data from the NMIMT system indicated that NPBPs were temporally isolated from other detectable transients in the same thunderstorms on time scales comparable to the times between normal lightning flashes in those storms, namely from several seconds to several tens of seconds. The occurrence of CIDs as solitary, isolated emissions stands in stark contrast to the majority of lightning emissions, which occur as trains of multiple pulses with interpulse periods ranging from a few microseconds to hundreds of microseconds. The apparent temporal isolation of CIDs will be discussed further in Chapter 6. Despite their occurrence as isolated pulses on short time scales, CIDs occurred during relatively intense periods of thunderstorm electrical activity.

Figure 4.17 shows time histories for each of the three storms within the context of cloud-to-ground lightning activity as reported by the National Lightning Detection Network. For each of the three storms, geographical subsets of the NLDN flash-level data were formed to show cloud-to-ground lightning activity within that storm. The flashes were placed in 10-minute time bins to show variations in flash rate throughout the lives of the storms. In the figure, negative cloud-to-ground flashes are represented by the low, gray bars. Positive cloud-to-ground flashes are represented by the high, black bars. Occurrences of CIDs are indicated by numbers on the tops of columns. The numbers indicate how many NPBPs were recorded in the corresponding time bins. Note that the absence of CID observations in the columns of Figure 4.17 does 

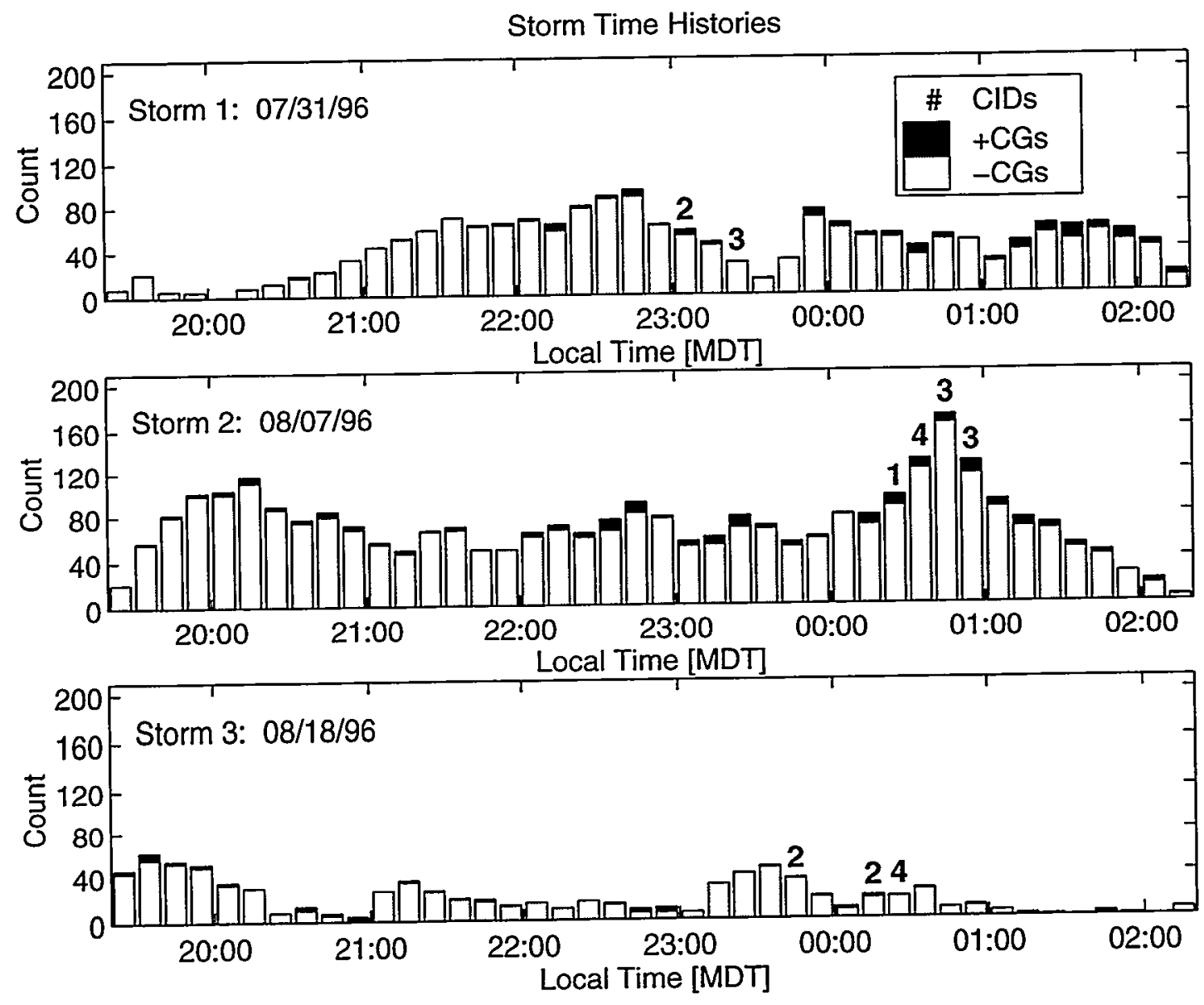

Figure 4.17: Plots of recorded $\mathrm{CD}$ activity and cloud-to-ground lightning activity as a function of time during the three storms. 
not necessarily mean that positive bipolar pulses did not occur, since recording stations were not operating during the entire time periods shown in the figure.

The time history plot for storm 1 shows that 5 CIDs occurred between 23:00 and 23:30 local time at the end of two hours of fairly intense lightning activity (5 to 9 CG flashes per minute). The storm was characterized by decreased activity following the occurrences of the recorded CIDs. One half hour after the last CID was recorded, the storm increased in CG flash intensity to the level that characterized the storm before the CDs were recorded. The storm ceased to produce much lightning activity approximately two hours later at 02:00 local time.

Storm 2 was by far the largest and most intense of the three storms analyzed in the study. The flash rate was 5 to 10 flashes per minute during the four hours preceding the recording of $11 \mathrm{CDs}$ that occurred between 00:30 and 01:00 local time. During the time when the CIDs were recorded, the CG flash rate jumped to between 10 and 18 flashes per minute. The storm ceased to produce CG lightning activity at about 02:00 local time, approximately 1 hour after the final $\mathrm{CDD}$ was recorded.

Storm 3 was the smallest of the storms. Eight CIDs were recorded between 23:30 and 00:30 local time. The events occurred during periods of relatively high intensity within the storm. The NLDN CG flash rate of 2 to 4 flashes per minute was quite low compared to rates observed by the NLDN during the other two storms when the CIDs were recorded.

Figure 4.17 shows that CIDs occurred during relatively active periods of cloudto-ground lightning activity within their respective storms. A high absolute flash rate does not appear to be a required condition or an indicator for the occurrence of CIDs, 
since only 2 to 4 flashes per minute were observed during storm 3 (although this is still a respectable flash rate).

\subsection{RADAR REFLECTIVITY OBSERVATIONS}

In addition to being active in terms of cloud-to-ground electrical discharges, storms 1 and 3 were active in terms of precipitation during the time periods when CIDs were recorded. Radar reflectivity data for storms 1 and 3 were obtained from the Albuquerque NEXRAD weather surveillance radar. Radar reflectivity data were not obtained for storm 2. During storms 1 and 3 the radar used a volume coverage pattern typically used to observe distant storms. Full $360^{\circ}$ azimuth sweeps were performed at 9 elevation angles every 6 minutes. The data received included reflectivity, mean radial velocity, and spectrum width. Only the reflectivity data are presented in this paper. Figure 2.1 shows the location of the Albuquerque NEXRAD radar $(A B X)$ and the two storms for which radar data were obtained. Storm 1 was located $250 \mathrm{~km}$ northeast of the radar. Storm 3 was located $100 \mathrm{~km}$ east of the radar.

\subsubsection{Storm 3}

Figure 4.18 shows a time sequence of four PPI (plan position indicator) radar reflectivity images from storm 3 . Plotted on top of the images are symbols representing recorded CIDs and cloud-to-ground lightning strokes as reported by the 
Day 96231: 05:43 - 06:29 UT; Radar Images @ 3.4 Elevation Angle $Z_{e}[\mathrm{dBZ}]$

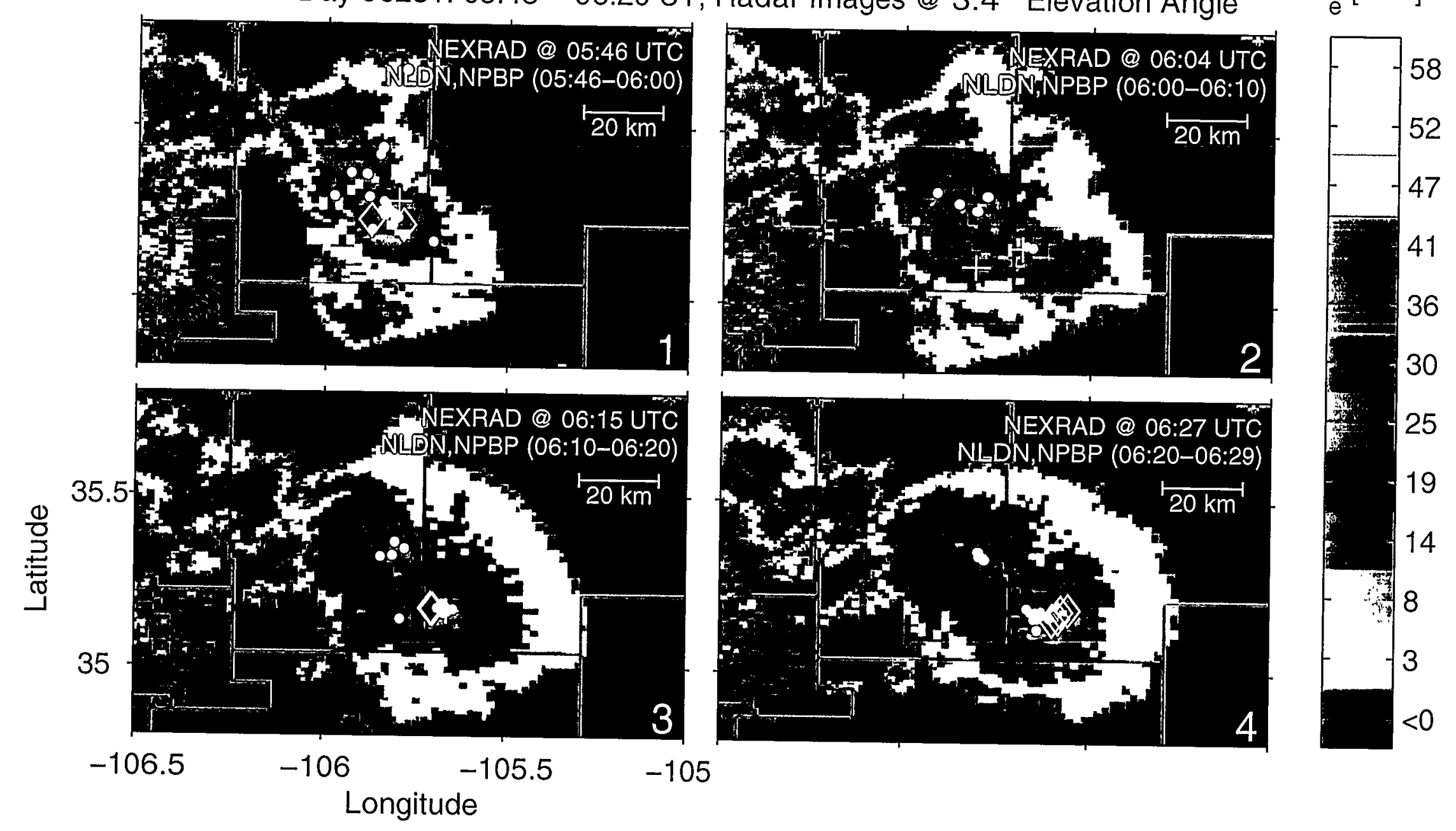

Figure 4.18: Plan view radar images of storm 3 as recorded by the Albuquerque NEXRAD radar. The radar sweep angle was $3.4^{\circ}$. 
NLDN. As in the previous plan view event location figures, CIDs are represented by diamonds, -CGs are represented by dots, and +CGs are represented by plus signs. The radar scans were acquired at 23:46, 00:04, 00:15, and 00:27 local time (MDT). Image 1 shows two CDs that occurred at 23:47 and 23:48 and all NLDN data for the time period from 23:46 to 00:00. Image 2 shows no CIDs and all NLDN data for the time period from 00:00 to 00:10. Image 3 shows two CIDs that occurred at 00:11 and 00:14 and all NLDN data for the time period from 00:10 to 00:20. Image 4 shows four CDs that occurred at 00:24, 00:25, 00:25, and 00:28 and all NLDN data for the time period from 00:15 to 00:29.

The radar elevation angle for each of the scans shown in Figure 4.18 was $3.4^{\circ}$. This elevation angle corresponds to an altitude of approximately $6.9 \mathrm{~km} \mathrm{MSL}$ at the position of the westernmost CID in panel 1 and an altitude of approximately $8.7 \mathrm{~km}$ MSL at the position of the easternmost CID in panel 4. A color scale showing effective radar reflectivity values in $\mathrm{dBZ}$ is shown at the right side of the figure.

The series of images indicates that the high reflectivity core of the storm was traveling toward the east-southeast at a speed of approximately $7 \mathrm{~m} / \mathrm{s}$. The locations of cloud-to-ground lightning flashes correspond well to regions of relatively high reflectivity (greater than $\sim 20 \mathrm{dBZ}$ ). Because the radar reflectivity images provide only a snapshot of precipitation echoes, but lightning activity is shown for time periods lasting from 9 to 14 minutes, exact studies of event locations with respect to reflectivity are not possible. However, it can be seen that the locations of cloud-toground flashes track well with the precipitation footprint of the storm. The 
observations of lightning activity and radar reflectivity are consistent with results from Krehbiel et al. [1984], Dye et al. [1986], Proctor [1991] and Maier et al. [1995].

CIDs also track well with the movement of the storm, but are more highly confined to a small region centered on the high reflectivity core. Note that source location uncertainty was greater in longitude $( \pm 4 \mathrm{~km})$ than in latitude $( \pm 200 \mathrm{~m})$ due to the location of the storm with respect to the recording stations. The elliptical location error ellipse for storm 3 was illustrated in Figure 4.13. Peak radar reflectivity in the core of the storm during the time period when the CIDs were recorded was greater than $52 \mathrm{dBZ}$. The bulk of the core was characterized by a peak reflectivity of greater than $40 \mathrm{dBZ}$.

Recall that the scans shown in Figure 4.18 were for a $3.4^{\circ}$ elevation angle only. Figures 4.19 and 4.20 show the PPI reflectivity scans for elevation angles of $4.3^{\circ}$ and $6.0^{\circ}$ respectively. In Figure 4.19 , the altitudes of the radar scan plane at the positions of the westernmost and easternmost CIDs were 8.3 and $10.5 \mathrm{~km} \mathrm{MSL}$ respectively. In Figure 4.20, the corresponding altitudes were 10.8 and $13.9 \mathrm{~km}$ MSL respectively. The time sequences of scans taken at all elevation angles during the time period when the CIDs were recorded show that the high reflectivity core of the storm was characterized by a peak reflectivity of $\geq 52 \mathrm{dBZ}$ up to an altitude of approximately 8 $\mathrm{km}$ MSL. Above $8 \mathrm{~km}$, reflectivity in the core of the storm dropped to a value of less than $20 \mathrm{dBZ}$ by an altitude of $11 \mathrm{~km}$ MSL. The height of the radar above mean sea level (MSL) is $1.8 \mathrm{~km}$. 


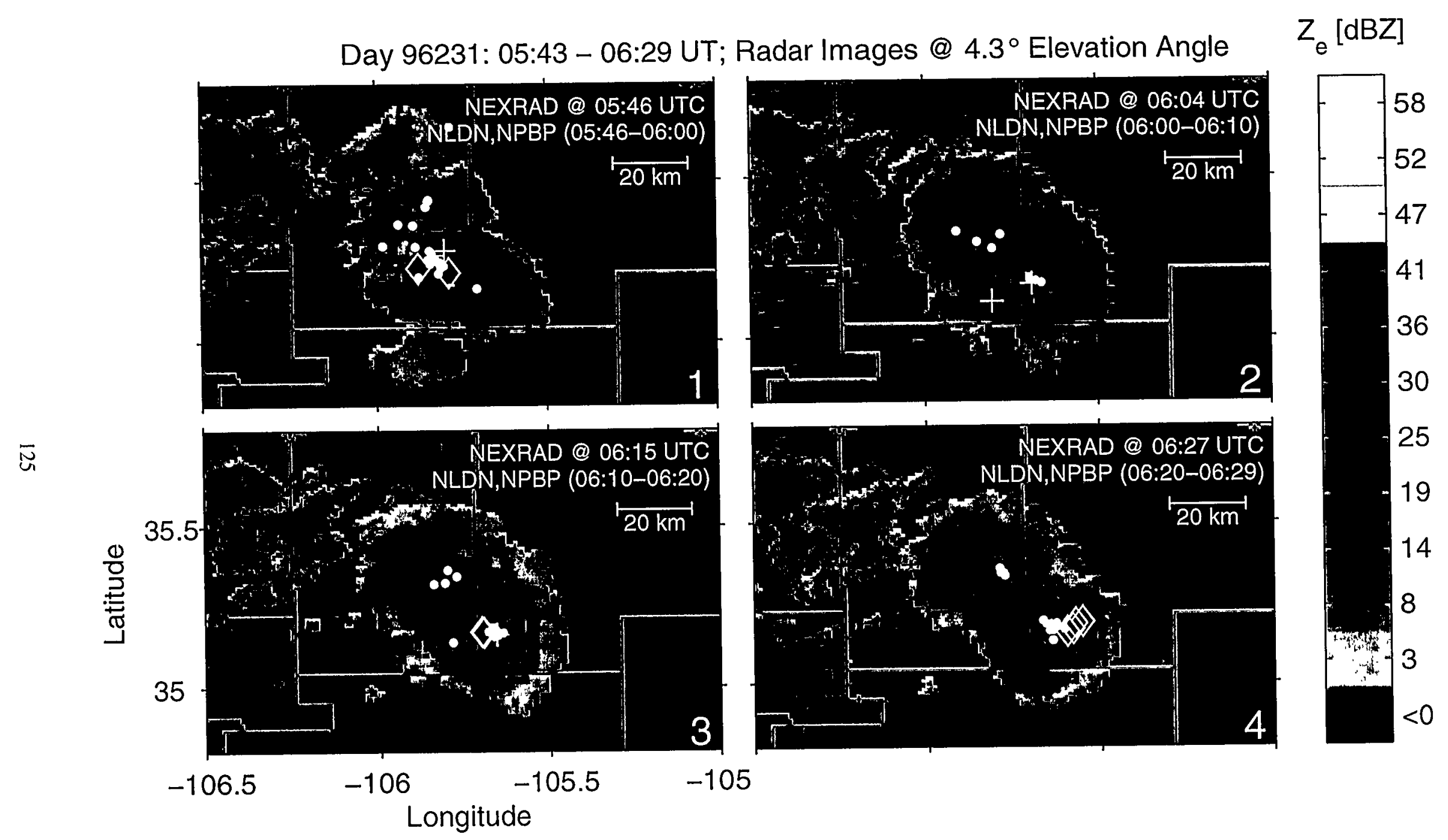

Figure 4.19: Plan view radar images of storm 3 as recorded by the Albuquerque NEXRAD radar. The radar sweep angle was $4.3^{\circ}$. 
Day 96231: 05:43 - 06:29 UT; Radar Images @ 6.0 Elevation Angle $Z_{e}[d B Z]$

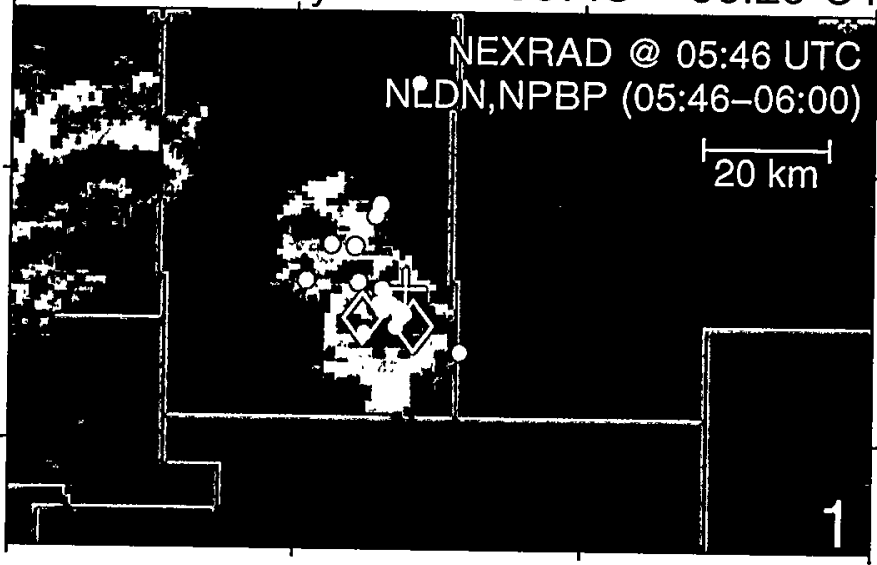
NEXRAD @ 06:04 UTC
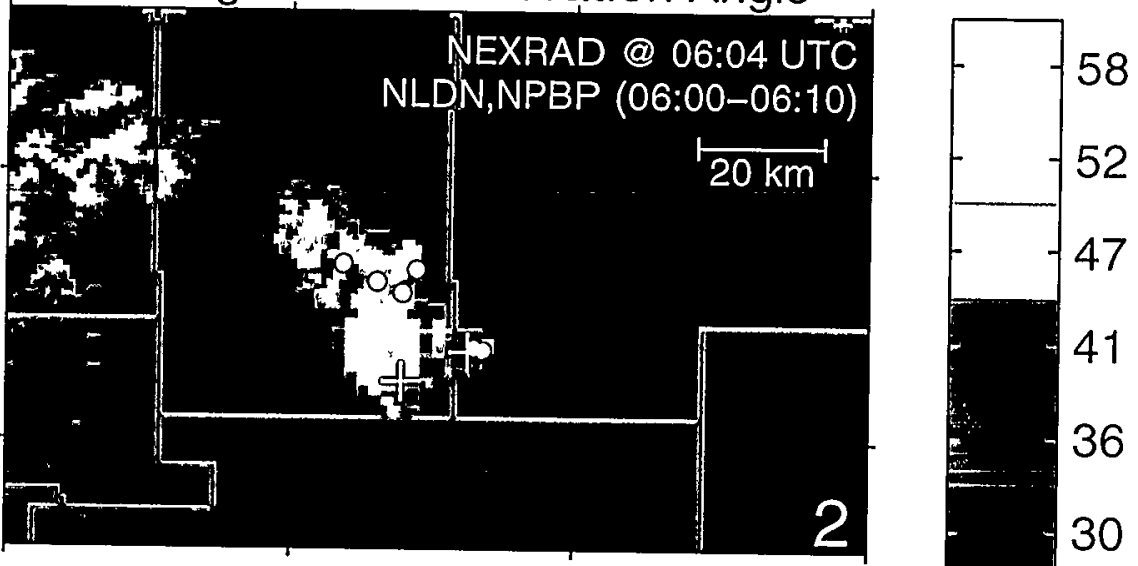

$\bar{\sigma}$

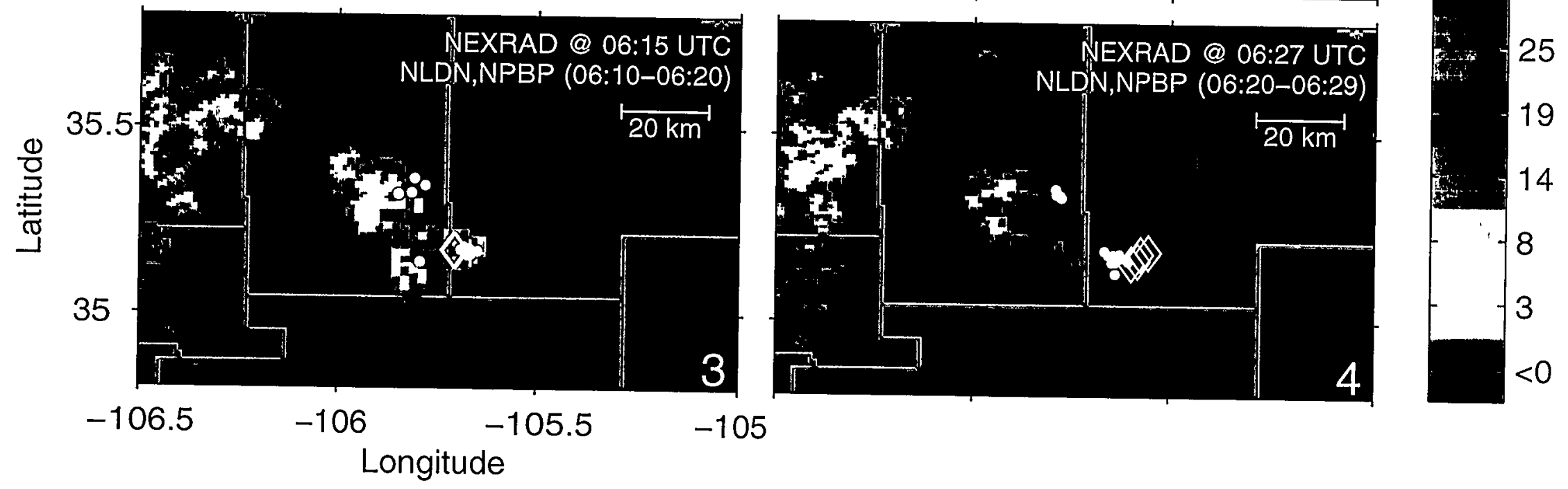

Figure 4.20: Plan view radar images of storm 3 as recorded by the Albuquerque NEXRAD radar. The radar sweep angle was $6.0^{\circ}$. 


\subsubsection{Storm 1}

The radar reflectivity results from storm 1 were similar to those from storm 3 . Figures 4.21, 4.22, and 4.23 show time sequences of PPI scans from storm 1 at elevation angles of $0.5^{\circ}, 1.5^{\circ}$, and $2.4^{\circ}$ respectively. The scans in the figures were acquired at approximately 23:07, 23:13, 23:19, and 23:25 local time (MDT) by the Albuquerque NEXRAD weather radar. Image 1 shows two CIDs that occurred at 23:07 and 23:08 and all NLDN data for the time period from 23:06 to 23:10. Image 2 shows no CIDs and all NLDN data for the time period from 23:10 to 23:16. Image 3 shows one CID that occurred at 23:21 and all NLDN data for the time period from 23:16 to $23: 22$. Image 4 shows two CIDs that occurred at 23:23, and 23:24 and all NLDN data for the time period from 23:22 to 23:25. In Figure 4.21, which shows the $0.5^{\circ}$ elevation angle sweeps, the altitudes of the radar scan plane at the positions of the southernmost and northernmost CIDs were 3.7 and $4.2 \mathrm{~km}$ MSL respectively. In Figure 4.22 , which shows $1.5^{\circ}$ elevation angle scans, the altitudes of the radar scan plane at the positions of the southernmost and northernmost CIDs were 7.5 and 9.0 $\mathrm{km}$ MSL respectively. In Figure 4.23 , which shows $2.4^{\circ}$ elevation angle scans, the altitudes of the radar scan plane at the positions of the southernmost and northernmost CIDs were 10.9 and $13.3 \mathrm{~km}$ MSL respectively.

The CID location errors for storm 1 were greater than for storm 3 due to the locations of the stations relative to the storm and their further distances from the

storm (>150 km for all field change and HF stations). As was illustrated in Figure 
Day 96213: 05:06 - 05:25 UT; Radar Images @ 0.5 Elevation Angle

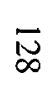

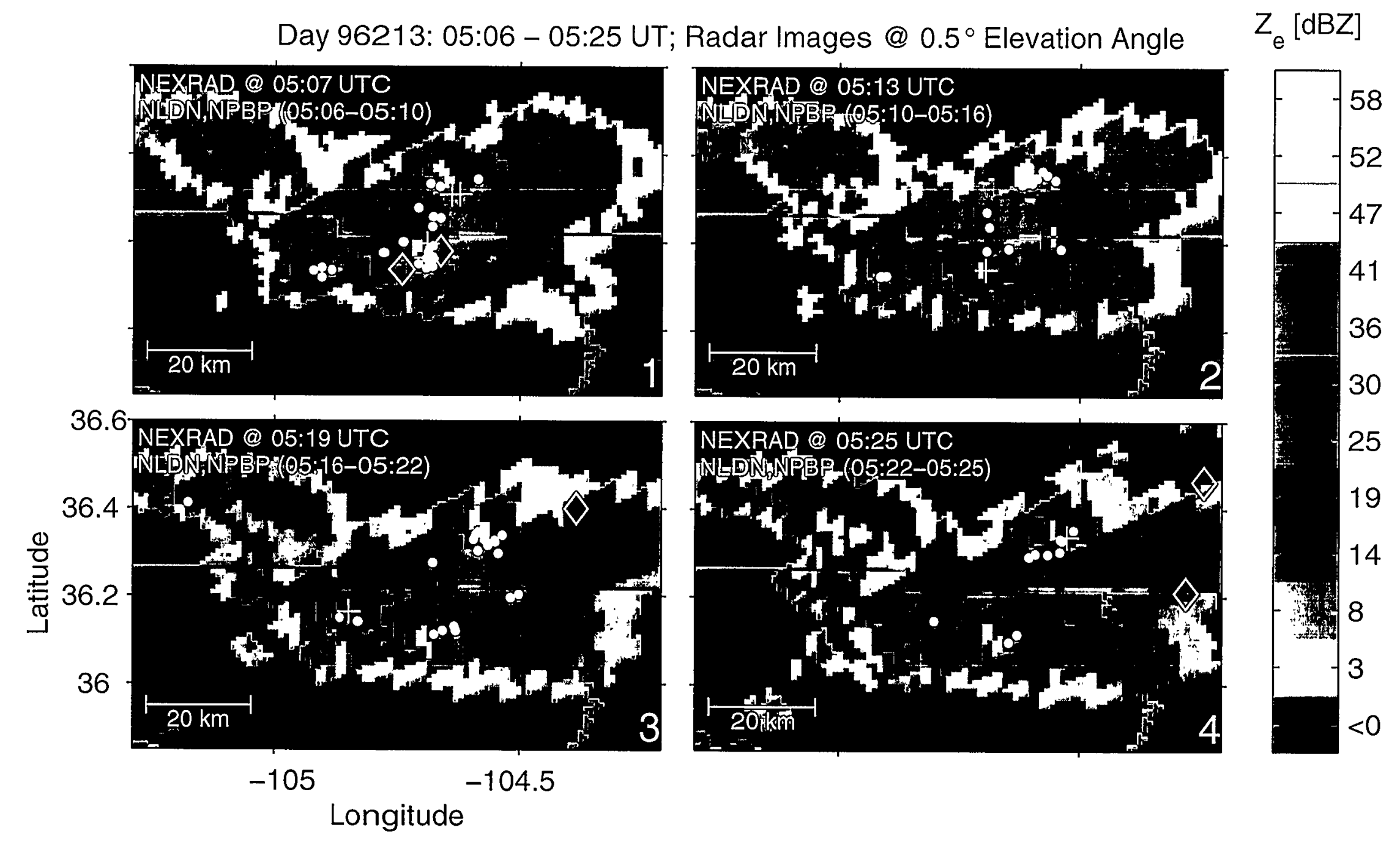

Figure 4.21: Plan view radar images of storm 1 as recorded by the Albuquerque NEXRAD radar. The radar sweep angle was $0.5^{\circ}$. 
Day 96213: 05:06 - 05:25 UT; Radar Images @ 1.5 Elevation Angle

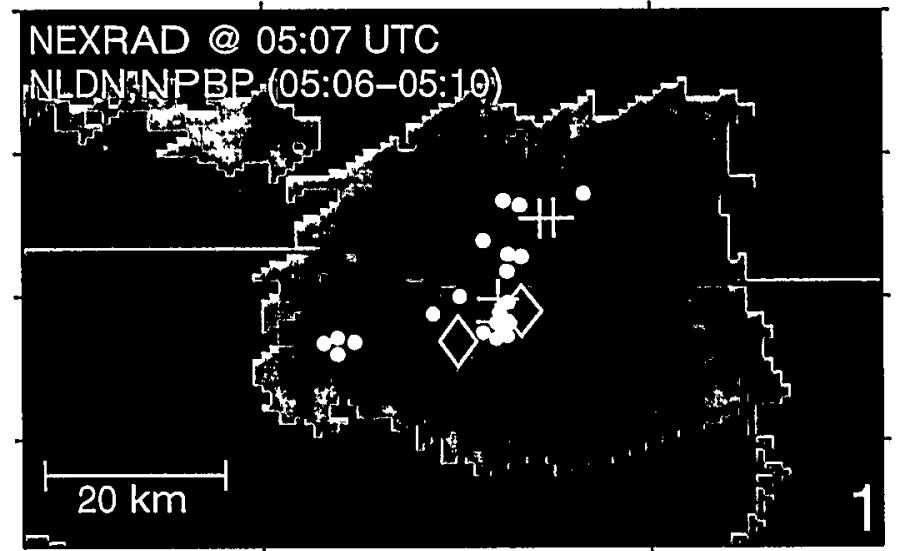

Nㅡㅇ

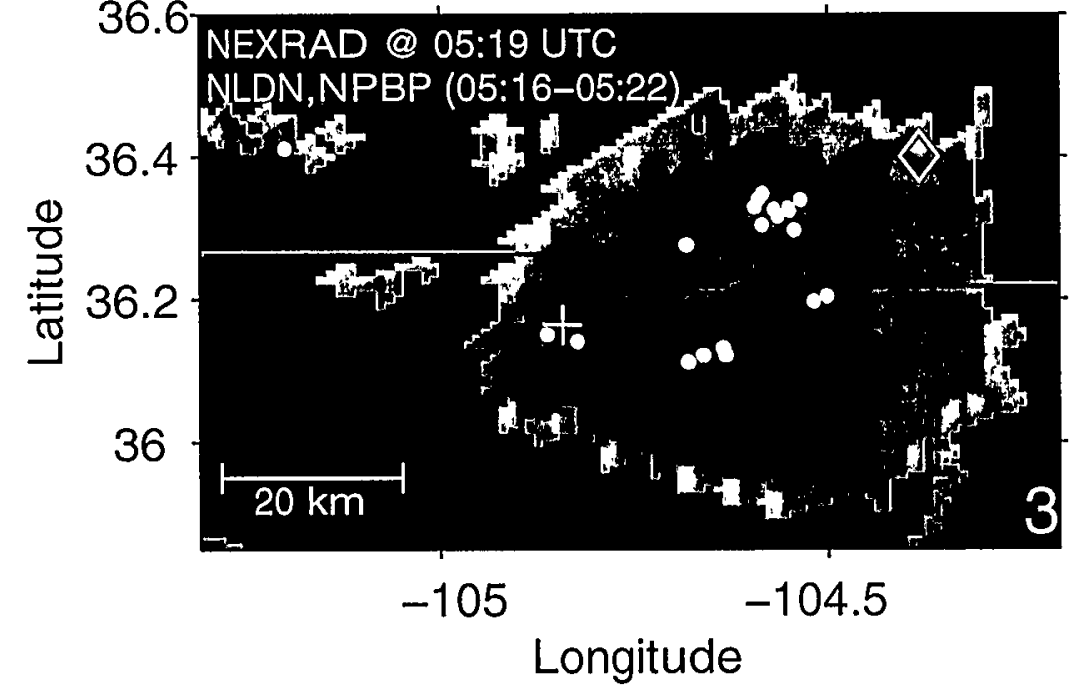

$Z_{e}[d B Z]$
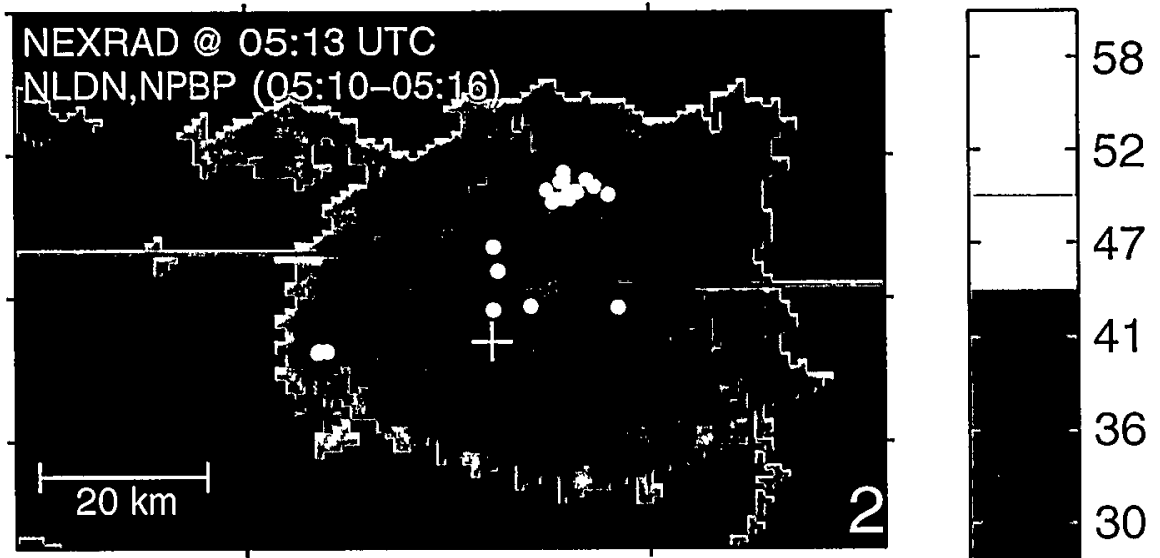

NEXRAD @ 05:25 UTC

NLDN,NPBP (05:22-05:25)

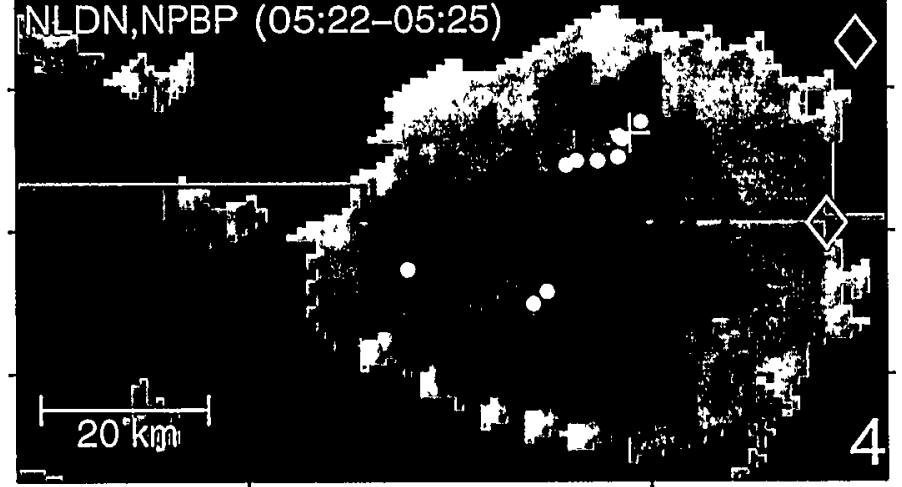

Figure 4.22: Plan view radar images of storm 1 as recorded by the Albuquerque NEXRAD radar. The radar sweep angle was $1.5^{\circ}$. 
Day 96213: 05:06 - 05:25 UT; Radar Images @ 2.4 Elevation Angle

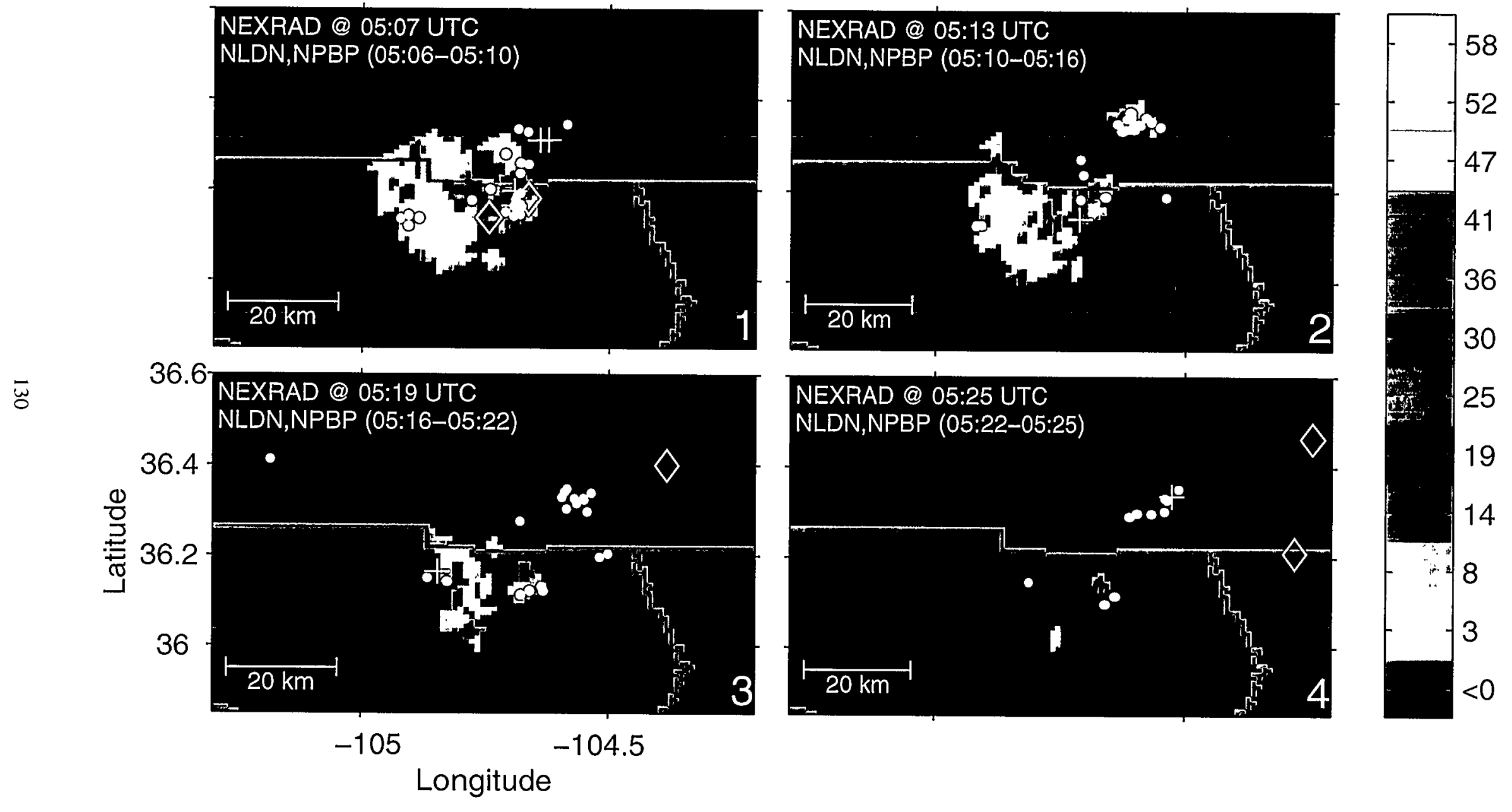

Figure 4.23: Plan view radar images of storm 1 as recorded by the Albuquerque NEXRAD radar. The radar sweep angle was $2.4^{\circ}$. 
4.9 , the $\mathrm{CID}$ source location uncertainty was greater than $\pm 20 \mathrm{~km}$ in a southwest by northeast direction and $\pm 4 \mathrm{~km}$ in the northwest by southeast direction. The radar data were also inferior to those obtained for storm 1 , since the radar was located at a distance of over $250 \mathrm{~km}$ from the storm.

As was the case for storm 3 , most of the CIDs recorded during storm 1 appear to have been associated with a region of high radar reflectivity. The large CID source location error uncertainty for storm 1 does not make it possible to determine whether the events were located within the high reflectivity core of the storm. The data do not rule out the possibility, except that the fifth event appears to have occurred $20 \mathrm{~km}$ from the others in the direction for which the spatial uncertainty was on the order of \pm $3 \mathrm{~km}$. As stated earlier, it is possible that a large timing error caused the discrepancy. The peak reflectivity in the core of the storm at the times when the CIDs were recorded was in excess of $47 \mathrm{dBZ}$ according to the NEXRAD data. 


\section{CHAPTER 5}

\section{OBSERVATIONS FROM TROPICAL CYCLONE FAUSTO}

One of the primary goals of the 1996 thunderstorm research campaign (and also a goal during previous years of observations) was to record an individual TIPP event using both Blackbeard and one or more recording stations located on the ground. During the summer of 1996, a large fraction of Blackbeard tasking was dedicated to arming the instrument over North America to search for TIPP events that might also be recorded by the ground-based electric field change and broadband HF arrays. The ALEXIS satellite typically makes four to six passes over the continental United States (CONUS) per day. During most of the thunderstorm campaign, three of the daily passes were dedicated to searching for TIPPs over CONUS. For each of the three passes, Blackbeard was armed for three one-minute time periods that began at fiveminute intervals. During each Blackbeard arm period, the instrument was able to record only the first event that exceeded its trigger threshold. Thus on any given pass, Blackbeard could record as many as three events, each separated from the next by approximately 5 minutes.

During the 1996 campaign, ground stations were operated in conjunction with every Blackbeard pass over North America. The stations were also operated at other times when they were used to make observations of local thunderstorms. Software 
was configured at each of the ground stations to arm the station two minutes prior to the start of the first Blackbeard arm period of the pass and to disarm the station one minute after the end of the final Blackbeard arm period of the pass. Thus ground stations were typically armed for 14 minutes during each pass. Unlike Blackbeard, ground stations were capable of recording many events during arm times. Typical ground station re-arm times were a fraction of a second for the field change meters and a few seconds for the broadband HF systems.

On two occasions in September of 1996, Blackbeard time tags for TIPP events occurred within $15 \mathrm{~ms}$ of time tags for events recorded by the LANL ground-based broadband HF data acquisition system (HF1). Fifteen milliseconds was used as a cutoff differential time of arrival (DTOA) to check whether events recorded by Blackbeard and a ground station could possibly be from the same source. The cutoff value was determined by characterizing the sensor/source configuration that produces the largest possible DTOA. This configuration occurs when the source occurs very close to the ground station that records the event and the satellite is as far as possible away from the source such that it can still receive VHF emissions from the source. For a source on the ground and under the assumption that radio waves travel in a straight line, the maximal distance occurs when the source is positioned on the limb of the earth as viewed from the satellite. For Blackbeard this distance is approximately $3300 \mathrm{~km}$. The corresponding delay is $11 \mathrm{~ms}$. Because atmospheric/ionospheric refractive effects cause the radio horizon to extend beyond the optical horizon, a cutoff delay of $15 \mathrm{~ms}$ was used, which corresponds to a path difference of $4500 \mathrm{~km}$. 
The two occurrences of sub-fifteen ms DTOAs between the Blackbeard TIPP events and the ground station event detections were the first such acquisitions since concurrent observation attempts had been begun three years earlier. The actual DTOA values for the events were 228 and $557 \mu$ s for the events that occurred on 11 and 19 September respectively. In each case the signal arrived at the ground station prior to its arrival at Blackbeard. As an initial indicator of the significance of the small DTOAs, note that during the three previous years, differences of less than 100 ms had occurred on only two occasions. In both of these cases the differences were greater than $50 \mathrm{~ms}$, far beyond that which is possible for signals emitted from the same source. The historically rare occurrence of small DTOA values qualitatively suggested that there was a high probability in each of the two cases that the signals were emitted by the same source.

From a statistical standpoint, the possibility that the sensors had recorded independent, unrelated events with such small differences in their times of arrival was also determined to be very unlikely. Based on typical Blackbeard arm-to-trigger times and typical ground-based event detection rates, it was estimated that the likelihood of "chance" detections from independent sources for each of the two cases was approximately 1 in 10,000 [Smith et al., 1997, a]. Knowing that the two events were excellent candidates for the first Blackbeard/ground station coincident detections, analyses were performed to locate the sources using two location methods. The analyses presented in the following sections show that locations determined using the two different techniques were consistent with each other and with data from another resource, the National Lightning Detection Network. These data have 
provided conclusive evidence of the association between large amplitude field change pulses (NPBPs) and TIPP/SIPP radio frequency pulses. The data have also provided further information about the conditions necessary for the production of CIDs and have provided more data on CID source heights.

\subsection{FIRST COINCIDENT EvENT: 11 SEPTEMBER 1996}

The first coincident detection was recorded at approximately $02: 30$ local time (MDT) on 11 September 1996. Figure 5.1 shows a spectrogram of the TIPP event that was recorded by Blackbeard. Note that a only a small fraction of the Blackbeard receiver bandwidth contained a signal. This is because a narrow, low-frequency, front-end analog filter setting (28 to $38 \mathrm{MHz}$ ) was used on Blackbeard for most of the TIPP searches over North America. The setting provides the greatest sensitivity to TIPP events in the Blackbeard low band (28 to $95 \mathrm{MHz}$ ) over CONUS. North American television and radio stations greatly reduce TIPP detectability in the upper portion of the low band (above $\sim 50 \mathrm{MHz}$ ). Each TIPP pulse in Figure 5.1 has a duration of a few microseconds. The pulses are separated by 27 microseconds.

Figure 5.2 shows the broadband HF waveform and HF spectrogram for the event as recorded by the ground-based LANL HF1 data acquisition system. The spectrogram exhibits multiple signals that propagated from the source to the receiver via reflections from the earth and multiple layers of the ionosphere. 


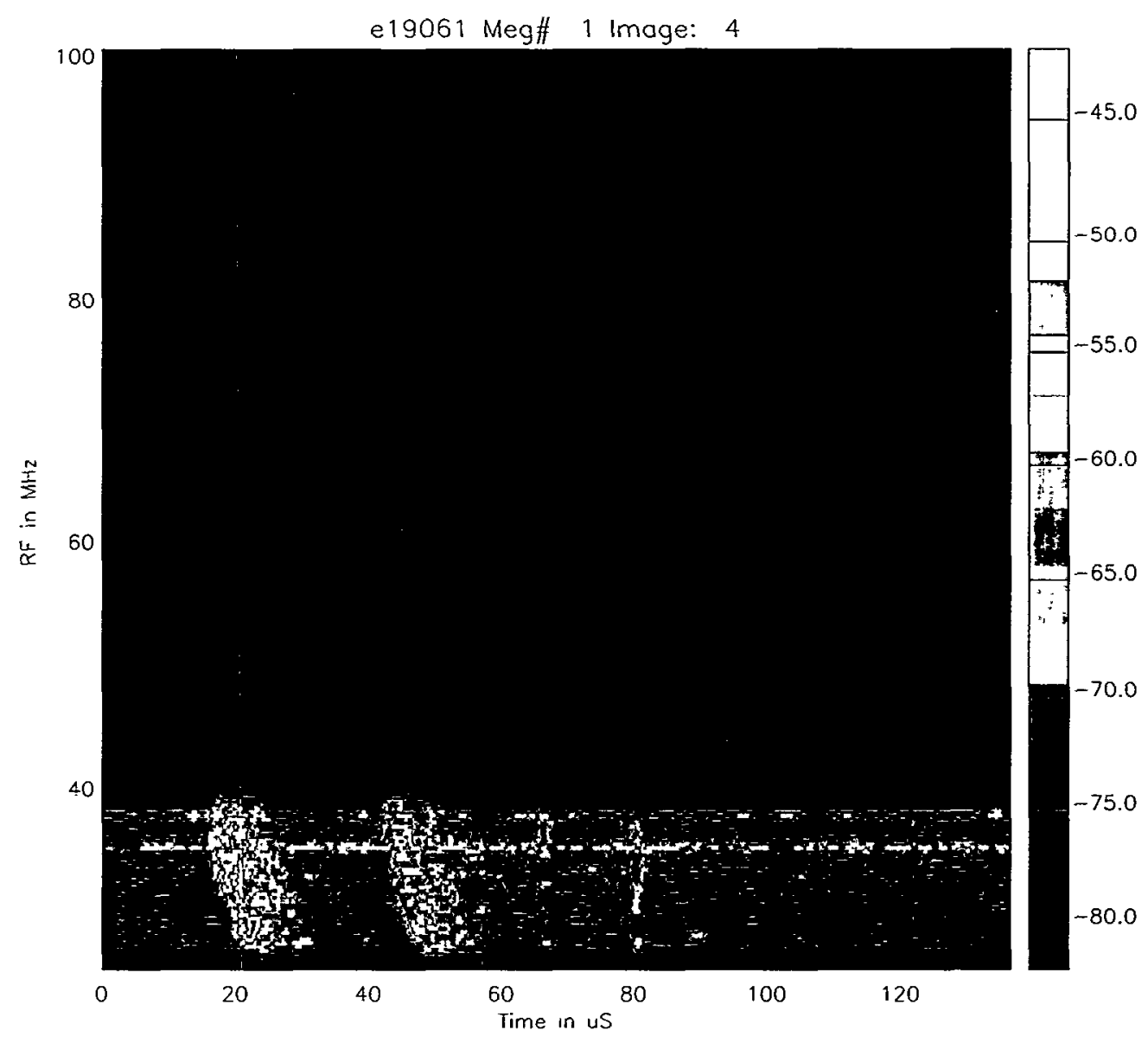

Figure 5.1: $\quad$ Spectrogram of the third TIPP event recorded by Blackbeard on 11 September 96 . The event was also recorded by the LANL broadband HF station (see Figure 5.2). 


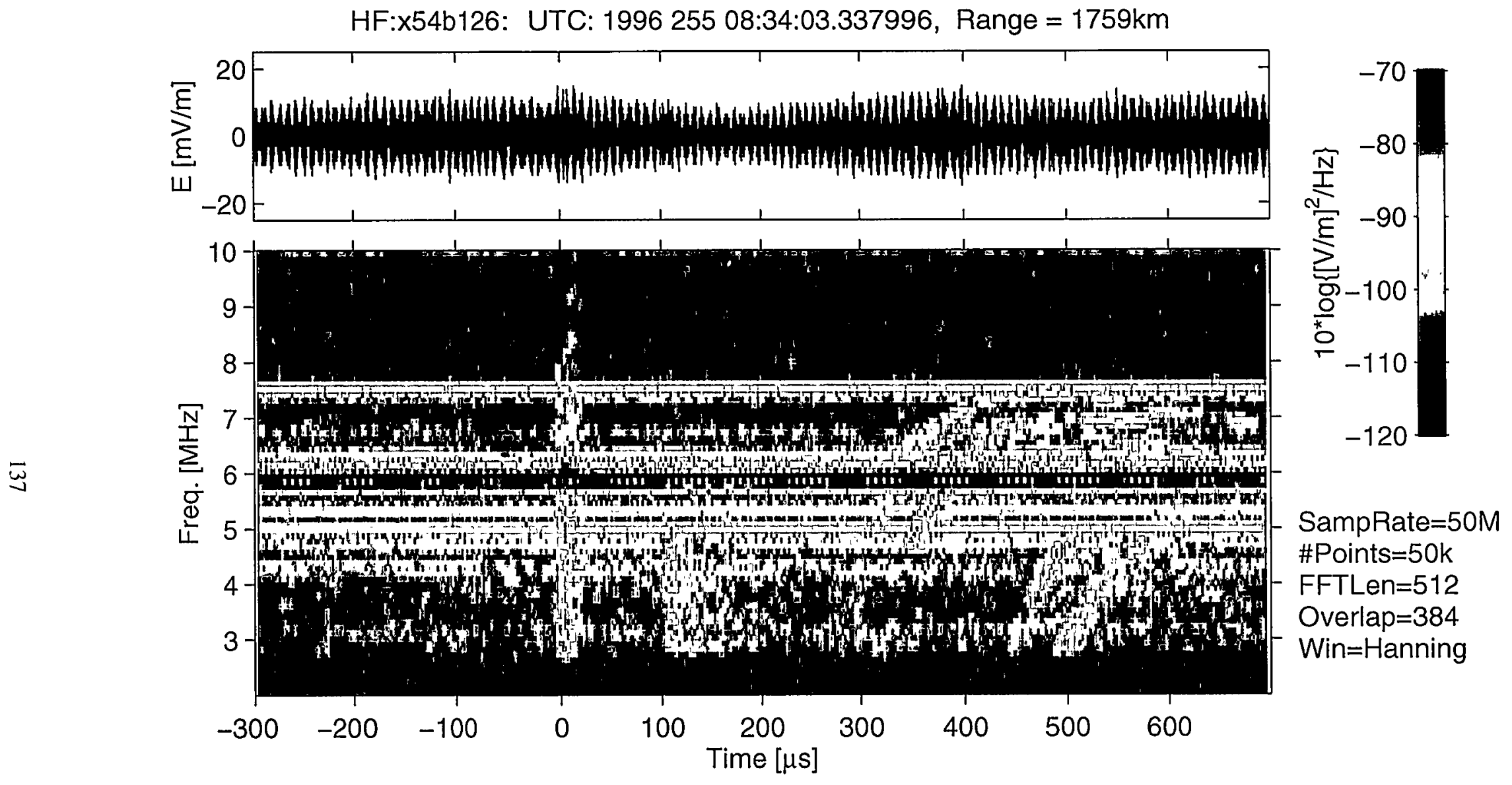

Figure 5.2: HF electric field waveform and spectrogram from a CID recorded on 11 September 1996. Multiple hops from multiple layers of the ionosphere were received. The event was coincident with the Blackbeard TIPP event pictured in Figure 5.1 . 
During the days when both observations were made (11 and 19 Sep.), the only ground resource that was recording waveforms was the Los Alamos broadband HF station (HF1). The Los Alamos field change station (FC1) was operating during both times and was being used as the trigger source for the HFl station, but was not configured to record waveforms.

Carrier signals between approximately 4.5 and $7.5 \mathrm{MHz}$ obscure some details of the recorded pulses in Figure 5.2. Figure 5.3 shows a "whitened" version of the figure in which the carrier signals have been removed through the use of digital filtering. The whitening filter operates on the raw broadband waveform in the following manner: A periodogram of the entire data record is formed using the fast Fourier transform (FFT). Then a vector of weights with the same length as the discrete frequency spectrum is created. Each weight in the weighting vector is inversely proportional to the waveform power in the corresponding frequency bin. Then the complex spectrum from the original FFT is multiplied by the square root of the weighting vector. The resulting spectrum has a flat (white) frequency response. The white output spectrum is processed using the inverse FFT to create a new time series with a flat frequency response. This time series is the output of the whitening filter. The filter removes CW signals from the original waveform by creating an output waveform with an equal amount of energy (integrated over the duration of the record) in each frequency bin. Thus frequency bins that have initially large power values (presumably from carrier signals) are multiplied by relatively small weights to assure that they have the same time-integrated power contributions as the other frequency steps. The method is not useful for characterizing waveform spectral characteristics, 


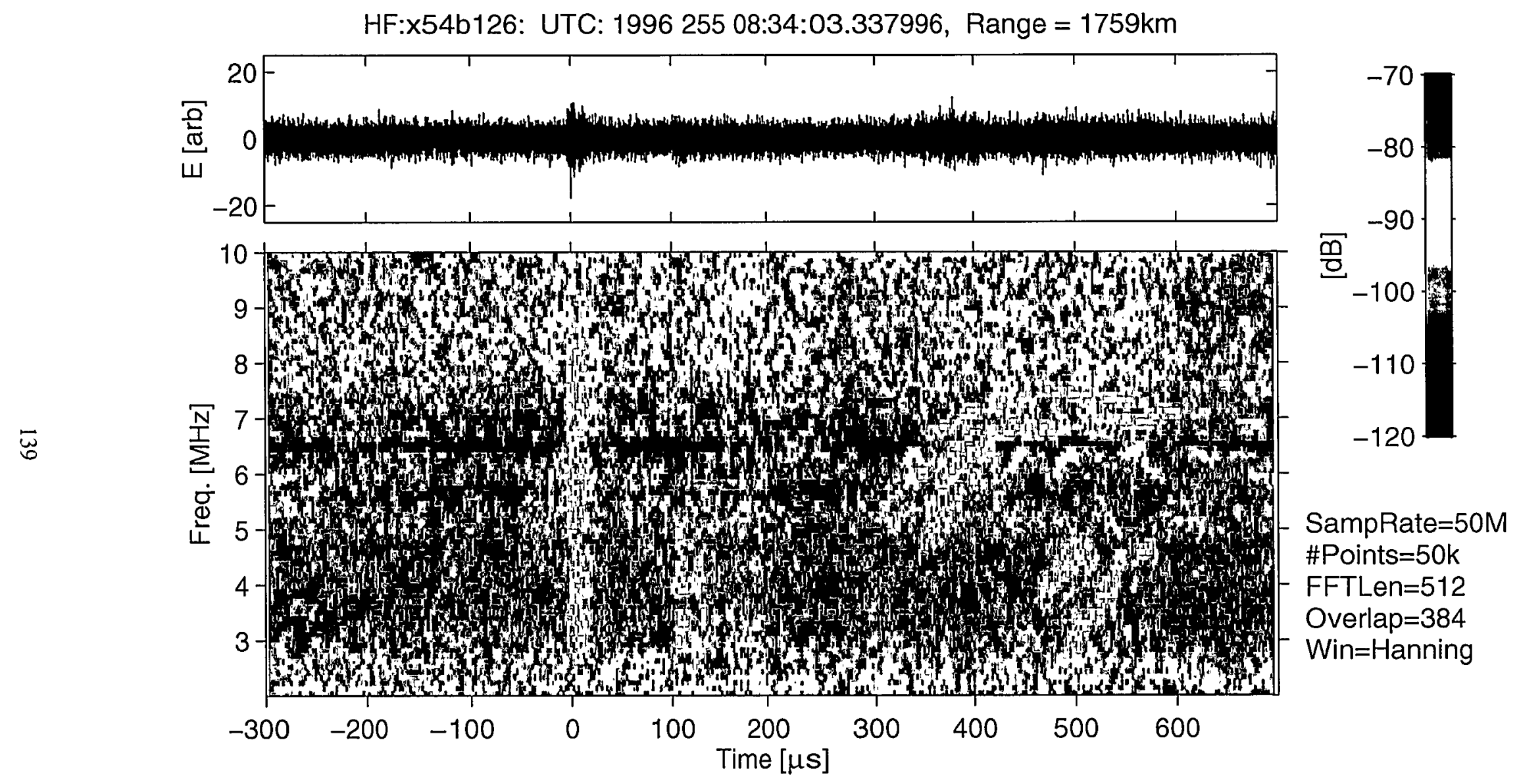

Figure 5.3: "Whitened" version of the waveform and spectrogram from Figure 5.2. 
but can create significant gain in waveform detectability in the presence of $\mathrm{CW}$ signals (as evidenced by comparing the time series and spectrograms in Figures 5.2 and 5.3). The method also makes some features in the spectrogram more obvious to the human eye. Horizontal dark bands that are evident in the whitened spectrogram of Figure 5.3 are drop-out regions where strong carriers were present in the pre-whitened spectrogram.

Two geolocation analysis techniques were used to determine the location of the source that produced the signals shown in Figures 5.1 and 5.2. In implementing the techniques, it was assumed that the one-source hypothesis (described in Section 1.1.1) provides the proper explanation as to why received pulses often occur in pairs. Thus, second pulses were regarded as reflections from the surface of the earth. The consistent results between the two location techniques provide further support for the hypothesis.

\subsubsection{Temporal Context of the Events Recorded on 11 September}

The TIPP event in Figure 5.1 was recorded by Blackbeard at 08:34:03 UTC (to the nearest second) on 11 Sep. 96. TIPP events were also recorded by Blackbeard at 08:24:17 and 08:29:01 on the same pass of the satellite over North America (times also rounded to the nearest second). The two TIPP events that were recorded prior to the one shown in Figure 5.1 are shown in Figures 5.4 and 5.5. The event in Figure 5.4 appears to consist of a single pulse rather than a pulse pair. However, the high signal strength of the pulse suggests that it was in fact a transionospheric pulse pair for 


\section{e19059 Meg\# 1 Imoge: 60}

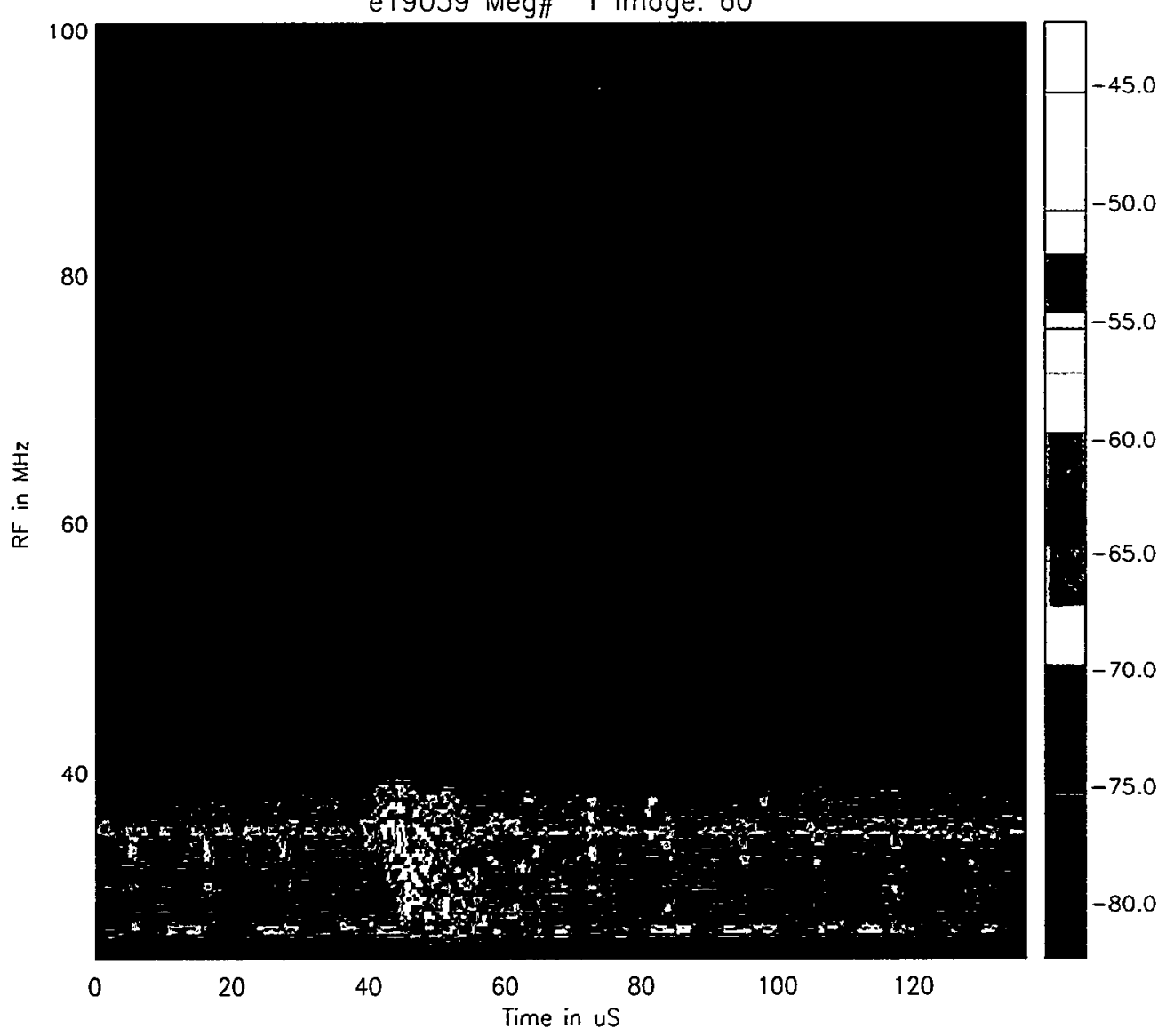

Figure 5.4: Spectrogram of the first TIPP event recorded by Blackbeard on 11 September 96. Because the source was on the limb of the earth (as viewed from Blackbeard), the two pulses are indistinguishable in the spectrogram. 


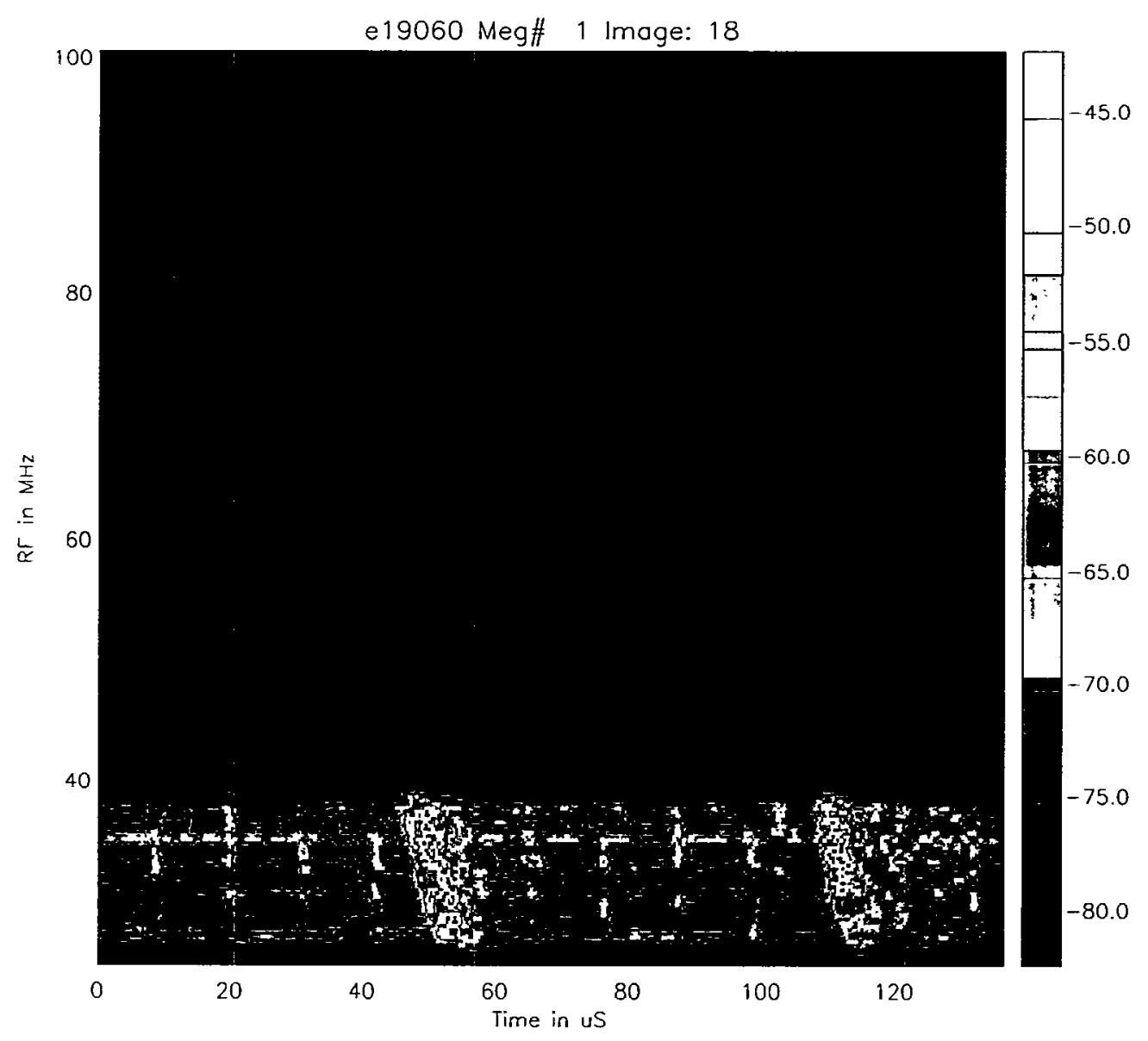

Figure 5.5: Spectrogram of the second TIPP event recorded by Blackbeard on 11 September 96. 
which the two pulses arrived at times so close together that the two pulses were indistinguishable. The longer duration of the emission in comparison to other TIPP pulses recorded during the pass provides support for this claim. The Blackbeard subsatellite locations and optical fields of view at the times of the three triggers are shown in Figure 5.6. ALEXIS was on a descending node as indicated by the approximate trigger times in the figure. During the pass, the Blackbeard receiver was armed for 1-minute time windows beginning at exactly 24,29 , and 34 minutes after 08:00 UTC on 11 Sep. Blackbeard was triggered 17, 1, and 3 seconds into the respective arm times. The relatively short time periods that were observed between Blackbeard arm times and trigger times suggest that there was a high event rate during the time of the satellite pass, although there is no way of knowing from the Blackbeard data alone whether a single storm or storm region produced the high rate or whether multiple storms or storm regions produced the triggers.

A high event rate was also observed by the ground-based HF system during the Blackbeard pass on 11 Sep. The system was armed for a 14-minute time window that began at 08:22 UTC, two minutes before the first Blackbeard arm time of the pass. As was standard procedure, the arm window was chosen to overlap all three Blackbeard arm periods. During the 14-minute window, the ground station recorded 111 events with spectrograms nearly identical to the one pictured in Figure 5.2. One of the events, which had a particularly high signal to noise ratio and was recorded 9 seconds before the coincident event, is shown in Figure 5.7. A whitened version of the event is shown in Figure 5.8. The remarkable similarity between the 111 spectrograms that were received during the arm period strongly suggests that all of the 
Blackbeard Sub-Satellite Points and FOVs for TIPPs on Day 96255

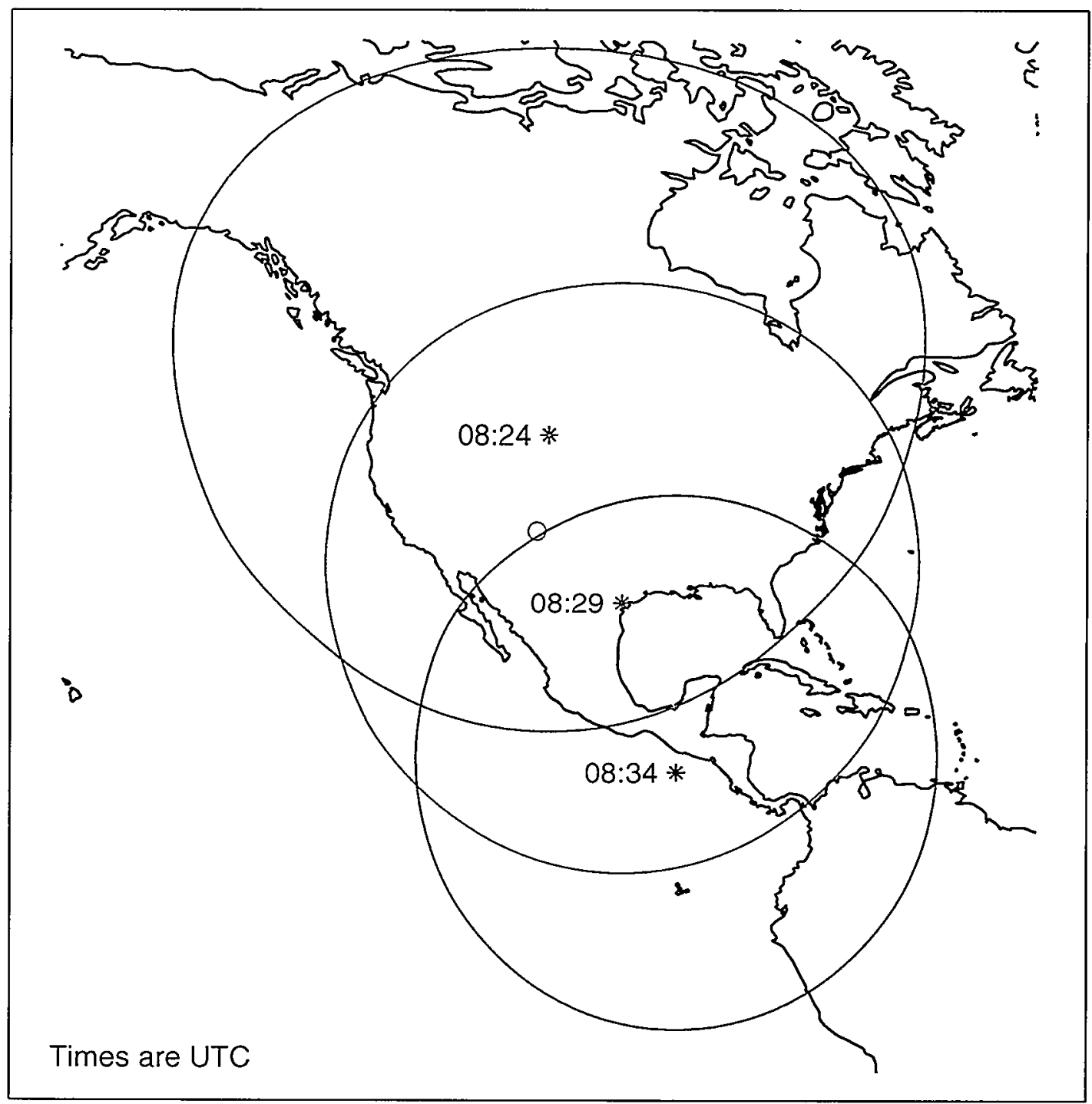

Figure 5.6: Blackbeard sub-satellite locations and fields of view during the times when the three TIPP events were recorded on 11 September 96 . The sub-satellite points are represented by colored asterisks. The correspondingly-colored lines show the location of the optical horizon as viewed from Blackbeard. 


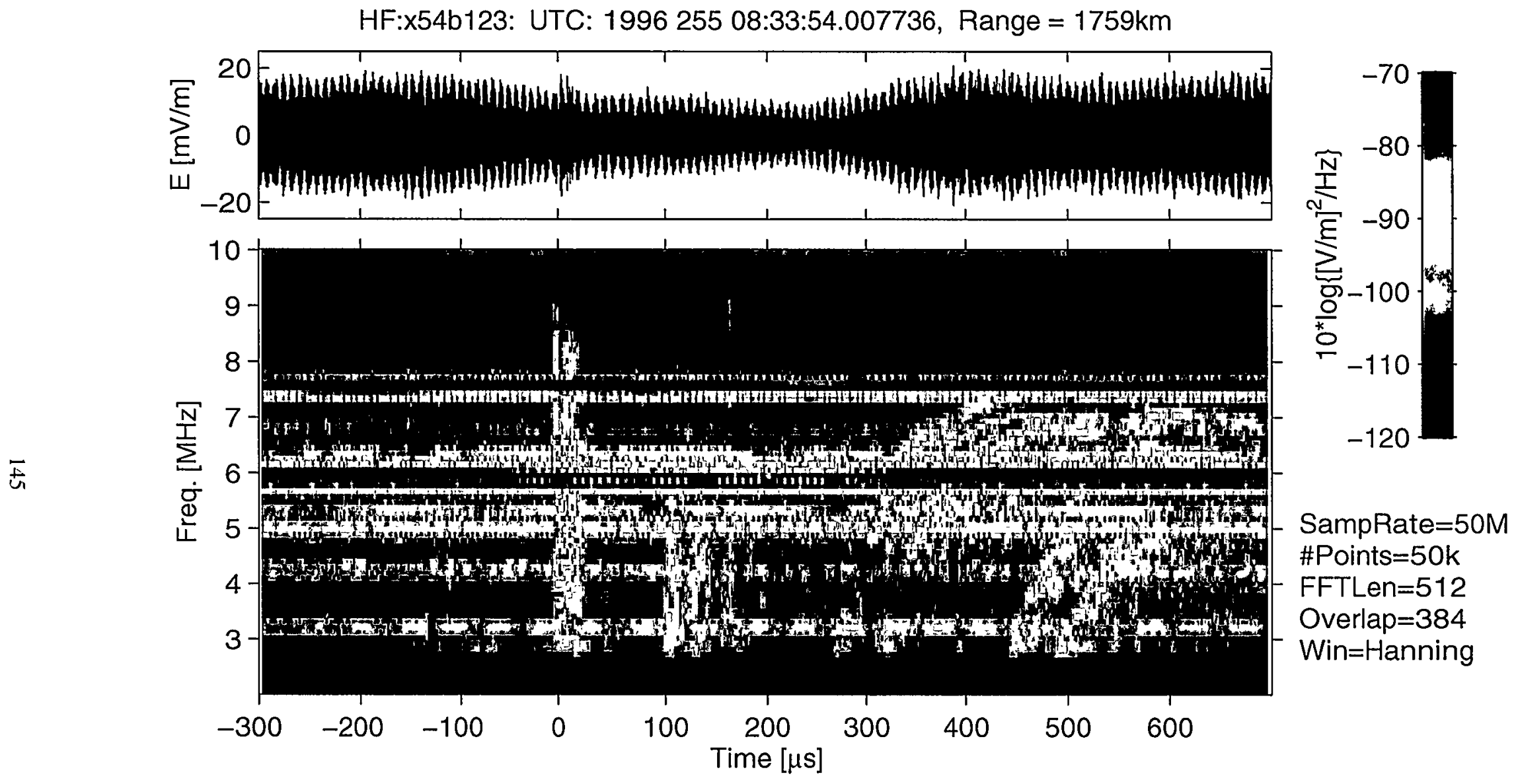

Figure 5.7: HF electric field waveform and spectrogram from a CID recorded on 11 September 1996. Multiple hops from multiple layers of the ionosphere were received. The event had a better signal-to-noise ratio than the event pictured in Figure 5.2 . 


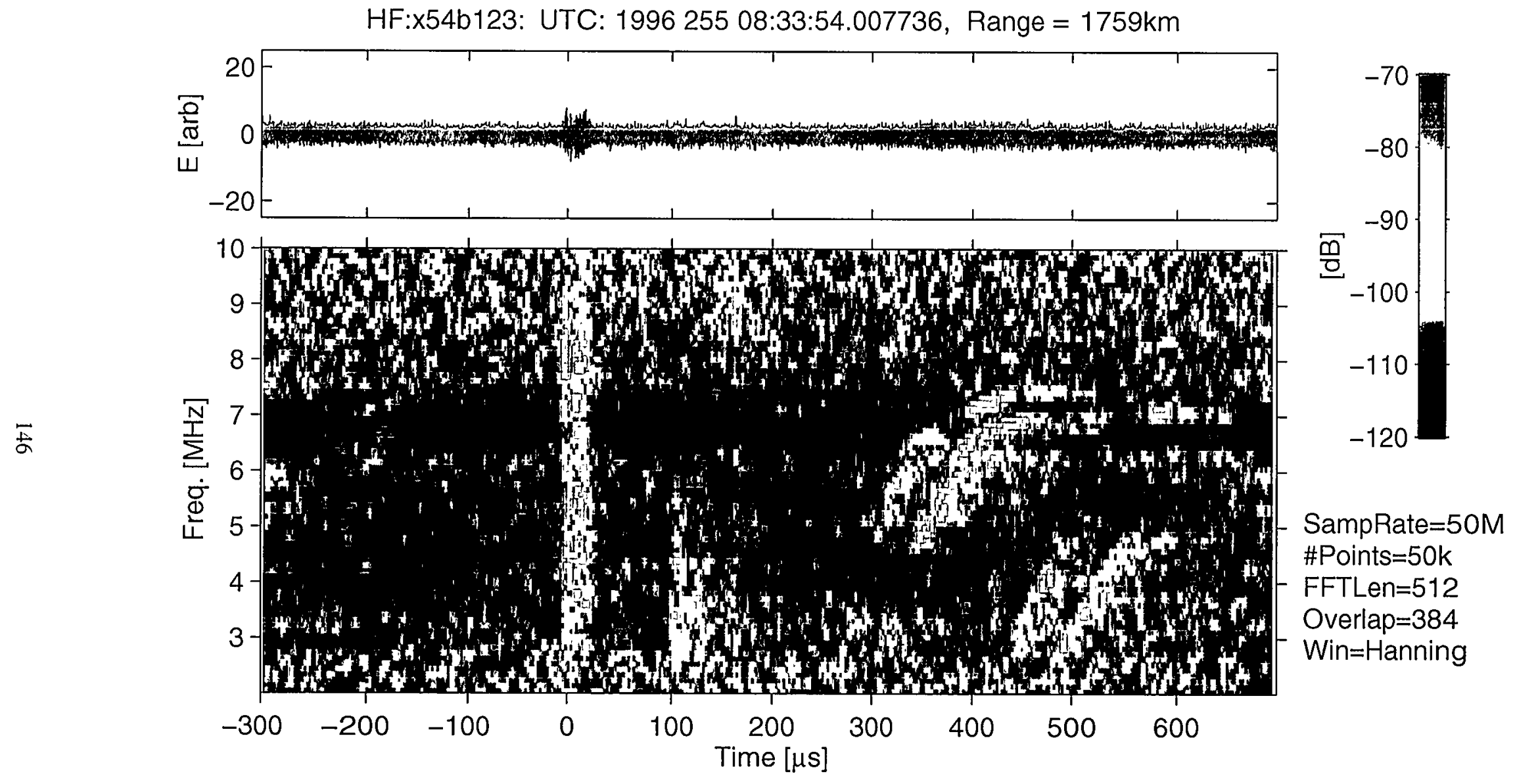

Figure 5.8: "Whitened" version of the waveform and spectrogram from Figure 5.7. 
signals originated from the same location. The average HF trigger rate for the distinct events during the arm window was 7.9 triggers per minute. The high trigger rate observed by the HF ground station is consistent with the high trigger rate inferred from the short trigger-to-arm times recorded by Blackbeard, providing further evidence that the two platforms observed events from the same location. Figure 5.9 illustrates the timing relationships between the arm times and trigger times for the ground-based station and Blackbeard during the satellite pass on 11 Sep. The green bar in the top panel shows the 14-minute time period during which the broadband HF data acquisition system was armed. The green plus symbols below the green bar show the times of occurrence of the $111 \mathrm{HF}$ events with matching spectrograms that were recorded. The lower red bars in the top panel show the three 1-minute Blackbeard arm times. The red plus symbols show the times of occurrence of the Blackbeard TIPP events. Recall that Blackbeard can record only one event during each arm period. The lower three panels of Figure 5.9 show magnified sections of the timeline pictured in the upper panel. The magnified sections are centered on the Blackbeard arm periods. The actual coincident event occurred during the third Blackbeard arm time.

\subsubsection{HF Propagation Model}

The multiple features evident in the ground-based HF spectrograms of Figures $5.2,5.3,5.7$, and 5.8 provide valuable information regarding the location of the source. Measurements of the relative timing between the multiple received 
Comparison of Blackbeard/Ground Station Events - DOY 255

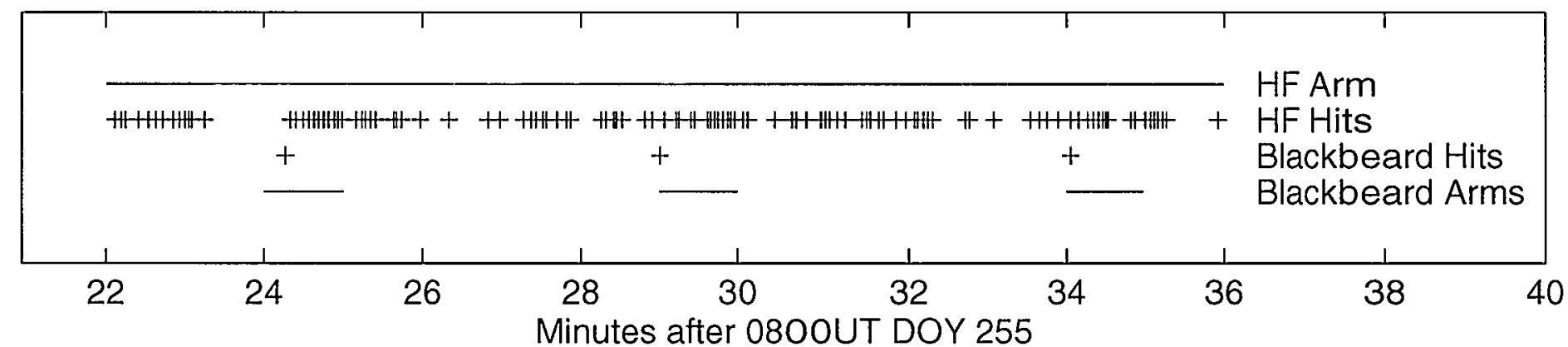

First Event

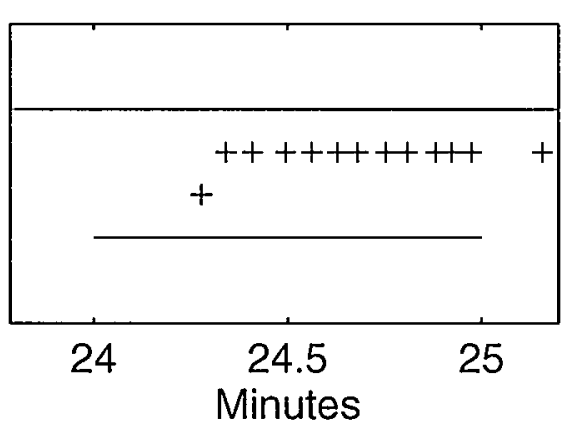

Second Event

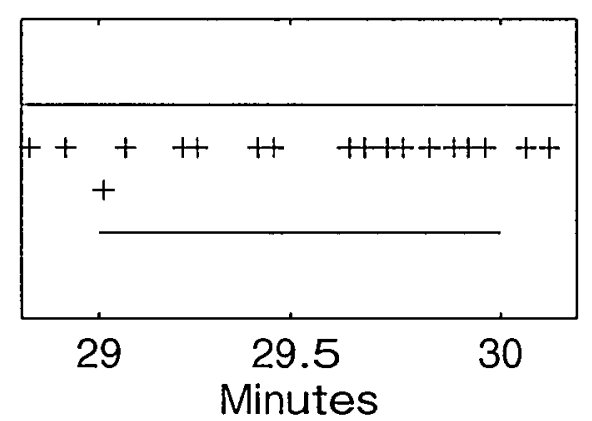

Third Event - BINGO

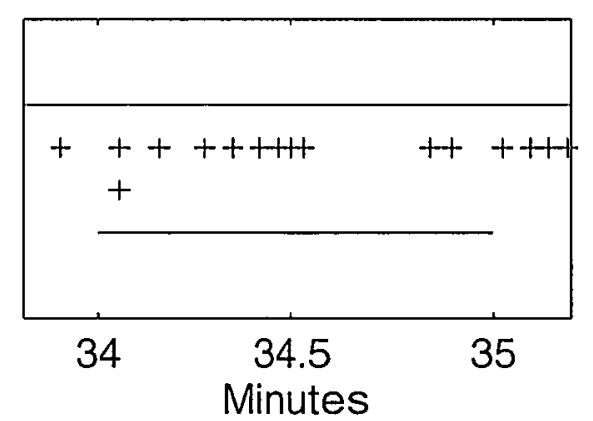

Figure 5.9: Time sequence of events on 11 September 1996. The plots show the arm periods and trigger times for Blackbeard and the ground-based HF system. The coincident event occurred during the third Blackbeard arm period. 
reflections allowed the distance to the source, the height of the source, and the virtual heights of the ionospheric E- and F- layers to be estimated. In order to make these determinations, an oblique ionospheric propagation code was developed to compute propagation delays for various propagation paths. In the ionospheric model it was assumed that ideal geometric reflections occurred from the surface of the earth and from single E- and F- ionospheric layers with fixed virtual heights. It was also assumed that the propagation paths followed straight lines. The model takes the curvature of the earth and ionosphere into consideration. The primary shortcoming of the model is the simplification of the reflection process. As in Chapter 3, the concept of virtual height was used to describe ionospheric reflections (which actually result from refraction). Factors such as the earth's magnetic field and electron gradients are also important, but probably secondary compared to simplification of the reflection process. Because propagation delays were measured at a single frequency, the largest discrepancy between the model and reality likely results from the fact that ionospheric virtual height is actually a function of angle of incidence for a given frequency. This effect is less important in consideration of the E-layer than the F-layer, because the Elayer has a relatively thin and dense profile so rays either reflect or pass through without experiencing much refractive bending.

Initially the propagation model was used to determine the propagation paths for each of the signals evident in the HF spectrograms. The spectrogram in Figure 5.8 was used to determine the delays of each of the signals because its signal to noise ratio was high in comparison to most of the other events that were recorded during the ground station arm time. Delays were determined at a frequency of $5 \mathrm{MHz}$ because 
all of the signals in the spectrogram either have distinguishable frequency content at that frequency or can be easily extrapolated to that frequency. Close inspection reveals that eight distinct signals are present in the spectrogram of Figure 5.8. The signals occur in pairs that begin at delays of $2,105,320$, and $503 \mu$ s at a frequency of $5 \mathrm{MHz}$ in the figure. The pair separations are $13,23,36$, and $54 \mu$ s respectively. In the initial interpretation step, general representative values for the virtual heights of the E- and F-layers (100 and $250 \mathrm{~km}$ respectively) and the height of the source $(10 \mathrm{~km})$ were plugged into the model. Then the range to the source was varied over a span of values from a few tens of $\mathrm{km}$ to $2000 \mathrm{~km}$. A good fit between the delays measured from the spectrogram and the delays output by the ionospheric model were obtained for a range of $1800 \mathrm{~km}$. It was determined that the various signals in the spectrogram corresponded to the following propagation paths:

The two signals in the first pair arrived at 2 and $15 \mu$ s in Figure 5.8 (and in Figures 5.2, 5.3, and 5.7). The first of the two was the 1-hop reflection of the source from the E-layer. Apparently the field change meter that was used to trigger the broadband HF system triggered off of the 1-hop field change signal and not the groundwave signal from the source. This is common for electric field change signals that propagate long distances. It has been observed that beyond 700 to $1000 \mathrm{~km}$, the amplitude of the reflected signals become greater than that of the groundwave signal. The second signal of the first pair was the 1-hop ionospheric reflection of the ground reflection of the source. Refer to the first panel of Figure 5.10 for an illustration of the propagation paths for the first pair of signals in the spectrogram. The two signals in the second pair arrived at 105 and $128 \mu \mathrm{s}$. These signals were the two-hop E-layer 


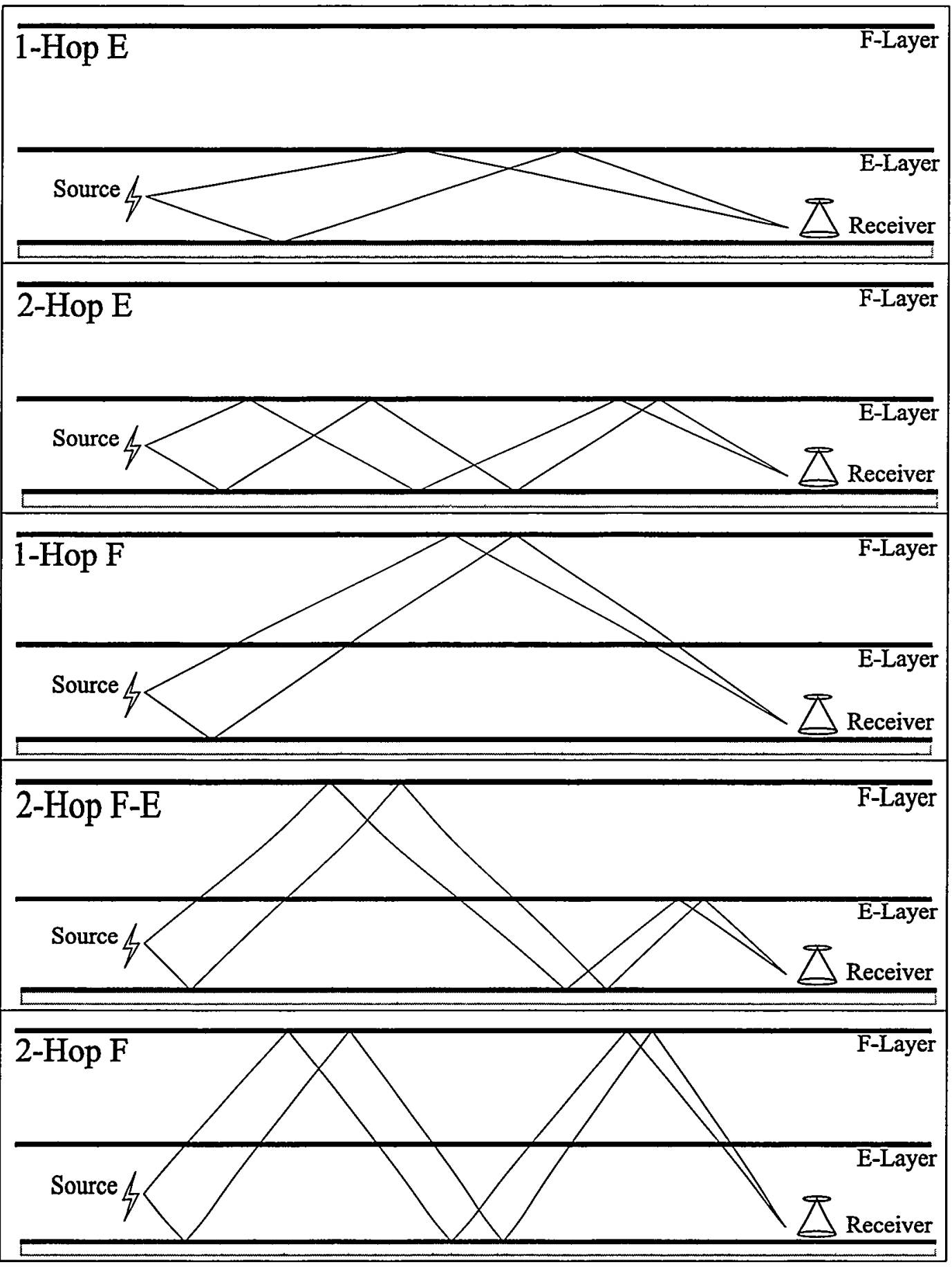

Figure 5.10: Illustrations of HF ionospheric propagation paths. 
ionospheric reflections of the source. Again the second signal of the pair was the initial ground reflection of the source. The second panel of Figure 5.10 illustrates the propagation paths. Neither pair of E-layer reflections exhibited noticeable dispersion, i.e. the signal times of arrival were not a function of frequency. This is an expected result of propagation from the E-layer [McNamara, 1991] and is consistent with this author's previous observations of signals reflected from the nighttime E-layer.

The signals comprising the third pair of emissions arrived at $320 \mu$ s and $356 \mu \mathrm{s}$ (at $5 \mathrm{MHz}$ ). The first signal was the 1-hop reflection of the source from the F-layer. The second signal of the pair was the 1-hop ground reflection of the source from the F-layer. The propagation paths for these signals are illustrated in the third panel of Figure 5.10. The emissions of the final pair of signals shown in Figure 5.8 arrived at 503 and $557 \mu \mathrm{s}$. At first it was assumed that the emissions were 2-hop F-layer reflections, but the signal times of arrival were $500 \mathrm{~ms}$ too early based on predictions from the ionospheric model. The addition of other possible propagation paths to the model showed that the delays matched those expected for two-hop propagation with one hop reflecting from the E-layer and one hop reflecting from the F-layer (although not necessarily in that order). These paths are illustrated in the fourth panel of Figure 5.10. Shown in the fifth panel are the 2-hop F-layer paths that would have arrived after the end of the $1 \mathrm{~ms}$ broadband $\mathrm{HF}$ recording.

Having determined the propagation paths, the model was reconfigured to minimize the differences between the observed propagation delays and the delays from the model as a function of the height of the source, the range to the source, and the two ionospheric virtual heights. The times of arrival of the first 1-hop E-layer 
reflection and the first 2-hop E-layer reflection were treated as fixed numbers. The Elayer and F-layer virtual heights ( $H E$ and $H F$ ) and the height of the source $(h)$ were varied throughout reasonable ranges. $H E$ was varied from 80 to $120 \mathrm{~km}$ in $0.5 \mathrm{~km}$ increments. $H F$ was varied from 200 to $300 \mathrm{~km}$ in $0.5 \mathrm{~km}$ increments. Source height, $h$, was varied from 2 to $30 \mathrm{~km}$ in $0.2 \mathrm{~km}$ increments. For each value of $H E, H F$, and $h$, the model returned the estimated times of arrival for each of the remaining six reflections. The times of arrival (TOAs) from the model were compared to the measured TOAs from Figure 5.8. The minimum mean square error over the threedimensional $(H E, H F$, and $h)$ grid search was found for the following values: $H E=97$ $\mathrm{km}, H F=277 \mathrm{~km}$, and $h=16.8 \mathrm{~km}$. The values for the E- and F-layer ionospheric virtual heights are consistent with values from Davies [1990] and are consistent with previous calculations based on data from oblique HF sounding receivers. Based on the numbers that produced the minimum timing errors, the distance from the source to the LANL HF station was $1760 \mathrm{~km}$. The model inputs, outputs, and errors are summarized in Table 5.1. Model outputs for the times of arrival of the two-hop Flayer signals are included in the table to show that the signals would have arrived after the end of the data record.

The ionospheric model, in combination with multiple, reflected HF signals from the source, allowed two important characteristics of the source to be determined: its height above ground level and its range from the LANL HF1 recording station. Having made these determinations, two independent location techniques were implemented to further pinpoint the sources of the recorded events by making use of data from the Blackbeard receiver. 
Curved-Earth Ionospheric Model

Frequency Used: $5 \mathrm{MHz}$

Earth Radius: 6378 km

\begin{tabular}{|l|l|l|l|}
\hline & Model Input: & Model Output: & Difference \\
\hline Measured Values: & & $2 \mu \mathrm{s}$ & $\mathrm{n} / \mathrm{a}$ \\
TOA for 1-Hop E & $2 \mu \mathrm{s}$ & $105 \mu \mathrm{s}$ & $\mathrm{n} / \mathrm{a}$ \\
TOA for 2-Hop E & $105 \mu \mathrm{s}$ & & $+0.9 \mu \mathrm{s}$ \\
1-Hop E Separation & $13 \mu \mathrm{s}$ & $13.9 \mu \mathrm{s}$ & $+1.2 \mu \mathrm{s}$ \\
2-Hop E Separation & $23 \mu \mathrm{s}$ & $24.2 \mu \mathrm{s}$ & $-3.3 \mu \mathrm{s}$ \\
TOA for 1-Hop F & $320 \mu \mathrm{s}$ & $316.7 \mu \mathrm{s}$ & $-2.4 \mu \mathrm{s}$ \\
1-Hop F Separation & $36 \mu \mathrm{s}$ & $33.6 \mu \mathrm{s}$ & $+5.9 \mu \mathrm{s}$ \\
TOA for 2-Hop E-F & $503 \mu \mathrm{s}$ & $508.9 \mu \mathrm{s}$ & $+2.5 \mu \mathrm{s}$ \\
2-Hop E-F Separation & $54 \mu \mathrm{s}$ & $56.5 \mu \mathrm{s}$ & \\
Varied Parameters: & & $1758.8 \mathrm{~km}$ & \\
E-Layer Virtual Height & $80-120 \mathrm{~km}$ & $97 \mathrm{~km}$ & \\
F-Layer Virtual Height & $200-300 \mathrm{~km}$ & $277 \mathrm{~km}$ & \\
Source Height & $2-30 \mathrm{~km}$ & $16.8 \mathrm{~km}$ & \\
Outputs: & & $5929.6 \mu \mathrm{s}$ & \\
Source Range (arc) & & $1076.5 \mu \mathrm{s}$ & \\
Time of Event & & $5 \mathrm{~s}$ & \\
TOA for 2-Hop F & & & \\
2-Hop F Separation & & & \\
\hline
\end{tabular}

Table 5.1: Inputs and outputs of ionospheric HF propagation model.

The first technique was based on the pulse separations observed for the three TIPPs recorded during the three Blackbeard arm times. The second technique was 
based on the measured differential time of arrival between the ground station signal and the Blackbeard signal for the event that was recorded by both platforms. The techniques and geolocation results are described in detail in the following sections.

\subsubsection{Location Technique One: Source Height and Pulse Separations}

The pulse separations for the Blackbeard TIPP events pictured in Figures 5.1 and 5.5 were 26 and 62 microseconds respectively. The Blackbeard event pictured in Figure 5.4 does not feature an obvious second pulse, but in other respects resembles the events pictured in Figures 5.1 and 5.5. Because the event is otherwise similar to the two TIPPs that were recorded approximately 5 and 10 minutes after the event, it was assumed that the single pulse was, in fact, a TIPP for which the separation between the two pulses was so small, that the two pulses were indistinguishable. This assumption is supported by the fact that the duration of the single pulse is longer than the those of the pulses in Figures 5.1 and 5.5.

As described in Chapter 1, if it is assumed that TIPP second pulses are reflections of singular pulses from the surface of the earth, then observed pulse pair separations from Blackbeard are functions of both the source height and the satellite look angle. This relationship was illustrated in Figures 1.3 and 1.4, which showed that the smallest separations are measured from low sources that occur near the limb and that the largest separations are measured from high sources that occur near satellite nadir. If, indeed, the three events in Figures 5.1, 5.4, and 5.5 were produced by sources in approximately the same location and at approximately the same altitude, then an 
analysis of the pulse separations and satellite locations given the estimated height of the source should indicate the source location.

A computer code was written to determine the locus of possible source locations on the earth that would produce a measured pulse separation given the height of the source and the location of the satellite at the time of the source emission. The loci take the form of circles centered on the sub-satellite positions. As in the oblique ionospheric model, geometric optics and a curved earth were assumed. The three Blackbeard TIPP detections on 11 Sep. were analyzed using the code. The satellite locations at each of the trigger times (which were shown in Figure 5.6) were computed from the ALEXIS two-line orbital elements, and were known to a reasonable degree of accuracy (within $20 \mathrm{~km}$ ). The source heights for the three events were assumed to lie within a range of $\pm 2 \mathrm{~km}$ from the estimate of $16.8 \mathrm{~km}$ AGL that was determined from the $\mathrm{HF}$ spectrograms as described in the previous section. Use of a range of possible heights rather than a single height caused the possible source loci to widen from circles into annuli.

Figure 5.11 shows the results of the analysis for the TIPP events recorded on 11 Sep. The red, green, and blue asterisks (which are labeled BB) show the Blackbeard sub-satellite locations at the times of each of the three TIPP events. The red, green, and blue annuli correspond to the projected possible source locations for each of the three events. The LANL HF1 station is represented by a cyan circle labeled 'HF1.' The large cyan circle around the station represents the estimated range to the source $(1760 \mathrm{~km})$ based on the ionospheric model described in the previous section. 


\section{Source Location Method One for TIPPs on Day 96255}

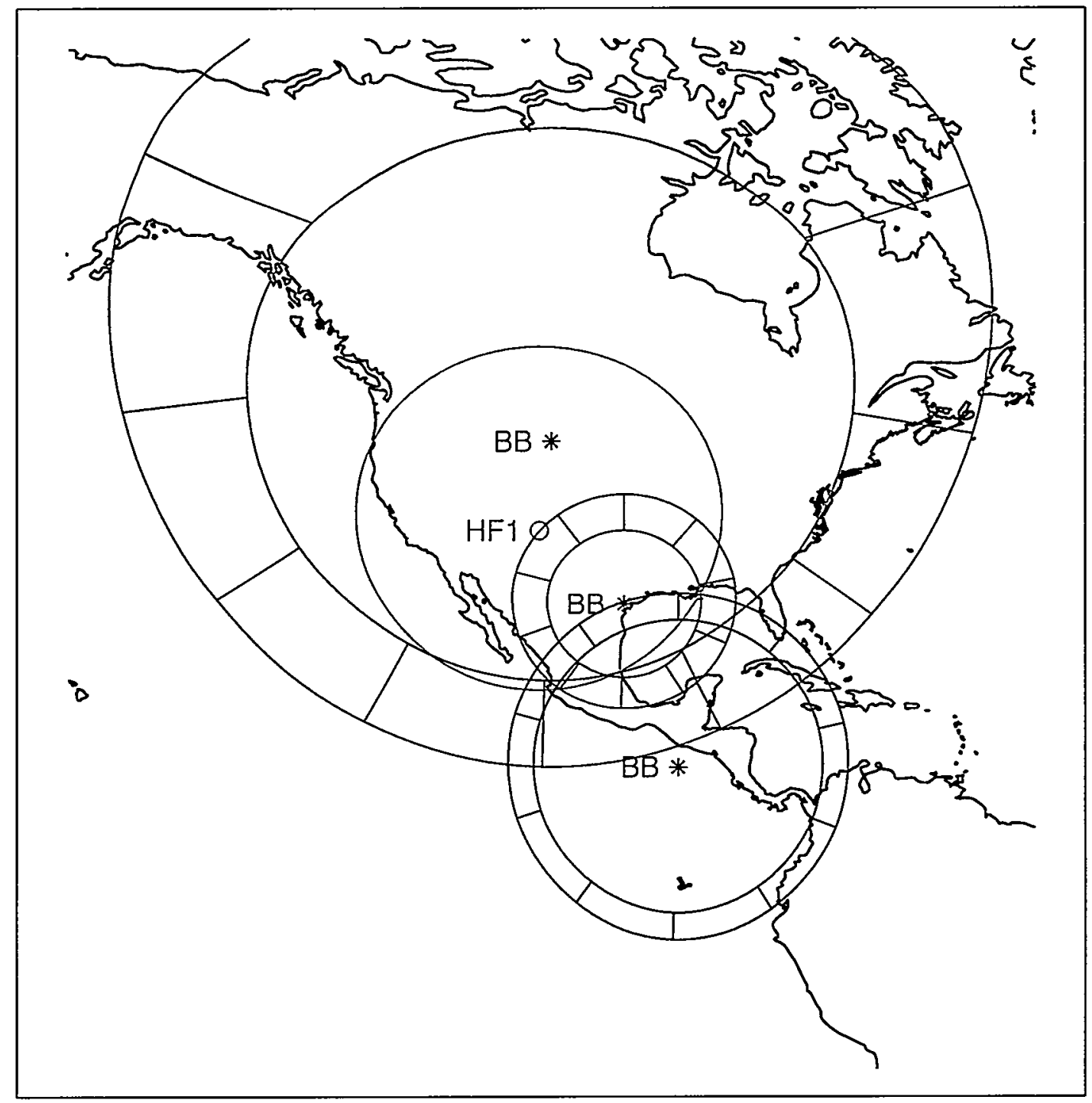

Figure 5.11: Illustration of source location method one for events recorded on 11 September 96. Colored (red, green, and blue) annuli show the most probable source locations for the three events based on the estimated source height and the pulse separations for the three TIPP events. The cyan circle shows the estimated range to the source from the HF1 recording station. 
The figure suggests that if the assumptions were valid, then there are two possible source regions. The regions are symmetric about the sub-satellite track and lie where the three annuli overlap. An expanded view of the two regions is shown in Figure 5.12, where the regions are represented as green triangles. One of the regions is on the west coast of Mexico near Punta Eugenia. The other is in the Gulf of Mexico approximately $500 \mathrm{~km}$ west of the central Florida peninsula. Using only the source height and pulse separations, it is not possible to resolve the ambiguity regarding which region produced the events. The ambiguity can be resolved using other data, however. One method of doing so is to make use of the range estimate to the source that was calculated from the ionospheric model. The estimated distance to the source from the LANL HF station (shown as a cyan circle in Figures 5.11 and 5.12) was $1760 \mathrm{~km}$. The distance to the potential source region in the Gulf of Mexico was approximately $2200 \mathrm{~km}$. The distance to the potential source region on the west coast of Mexico was approximately $1750 \mathrm{~km}$. The results from the model identify the region on the Mexican coast as the more likely source region. Further evidence in support of this source location is provided by subsequent analyses.

\subsubsection{Location Technique Two: Source Range and DTOA}

A second, independent method was used to determine the location of the single event that was recorded by both Blackbeard and the LANL HF ground station (HF1) on 11 Sep. In this method the range estimate to the source from HFl and the 
Source Location Method One for TIPPs on Day 96255

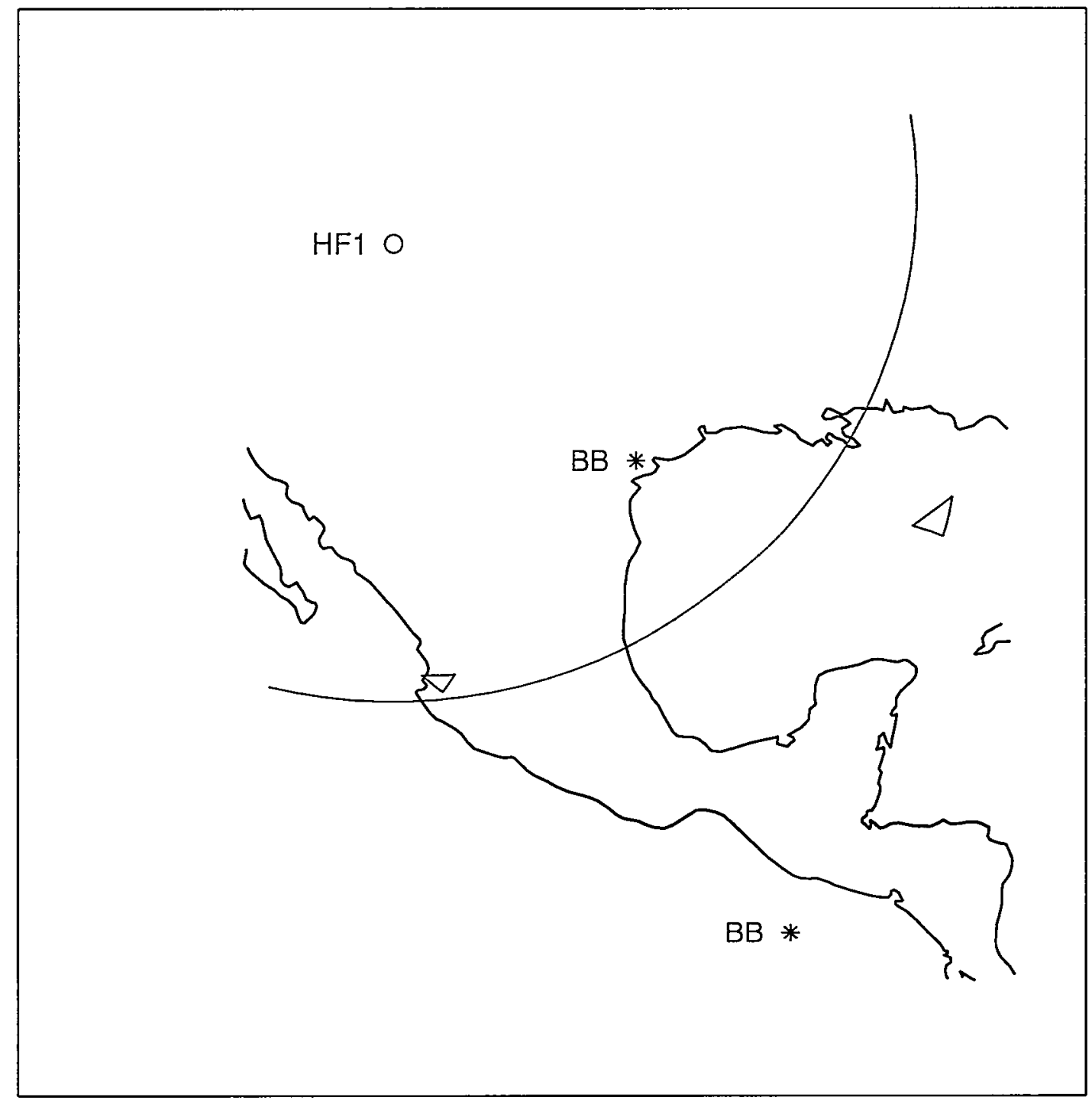

Figure 5.12: Illustration of the most probable source locations based on location method one. The green triangles show the overlapping portions of the three colored annuli from Figure 5.11. The close proximity of the cyan range circle to the westernmost triangle suggests that the events originated in the vicinity of the triangle. 
difference in the signal times of arrival between the satellite- and ground-based plat forms were used.

Based on the results of the ionospheric model presented in Section 5.1.2, the estimated range to the source from the LANL ground-based HF station was $1760 \mathrm{~km}$. From that information alone, the locus of possible source locations is restricted to a circle centered on the recording station with an arc radius of $1760 \mathrm{~km}$. Such a circle is depicted in cyan in Figure 5.13. The uncertainty in the range was estimated to be \pm $100 \mathrm{~km}$.

Using the differential time of arrival (DTOA) of the coincident signals received by HFI and Blackbeard, the source of the event that was recorded from both platforms was further pinpointed. In two dimensions, the locus of points, or isochrone, for which a constant DTOA relationship is satisfied between two recording points is a hyperbola. In three dimensions, the locus of points is one half of a twosheet hyperboloid (if the sign of the DTOA is unknown, then the locus of points is a full two-sheet hyperboloid). The hyperboloid is an isochronal surface, since a constant differential time of arrival relationship exists for all points on its surface. For the problem at hand, the intersection of the hyperboloid with the constant range circle on the surface of the earth defines a possible source location or possible source locations. In general, two solutions will exist. They will be symmetric about the line between the ground station and the sub-satellite point.

The event that was recorded by both Blackbeard and the LANL HF station on 11 Sep. was detected by Blackbeard $228 \mu$ s after it was detected by the ground station. As stated in Chapter 2, the uncertainty in the ground station timing was $2 \mu$ s and the 
Source Location Method Two for TIPP on Day 96255

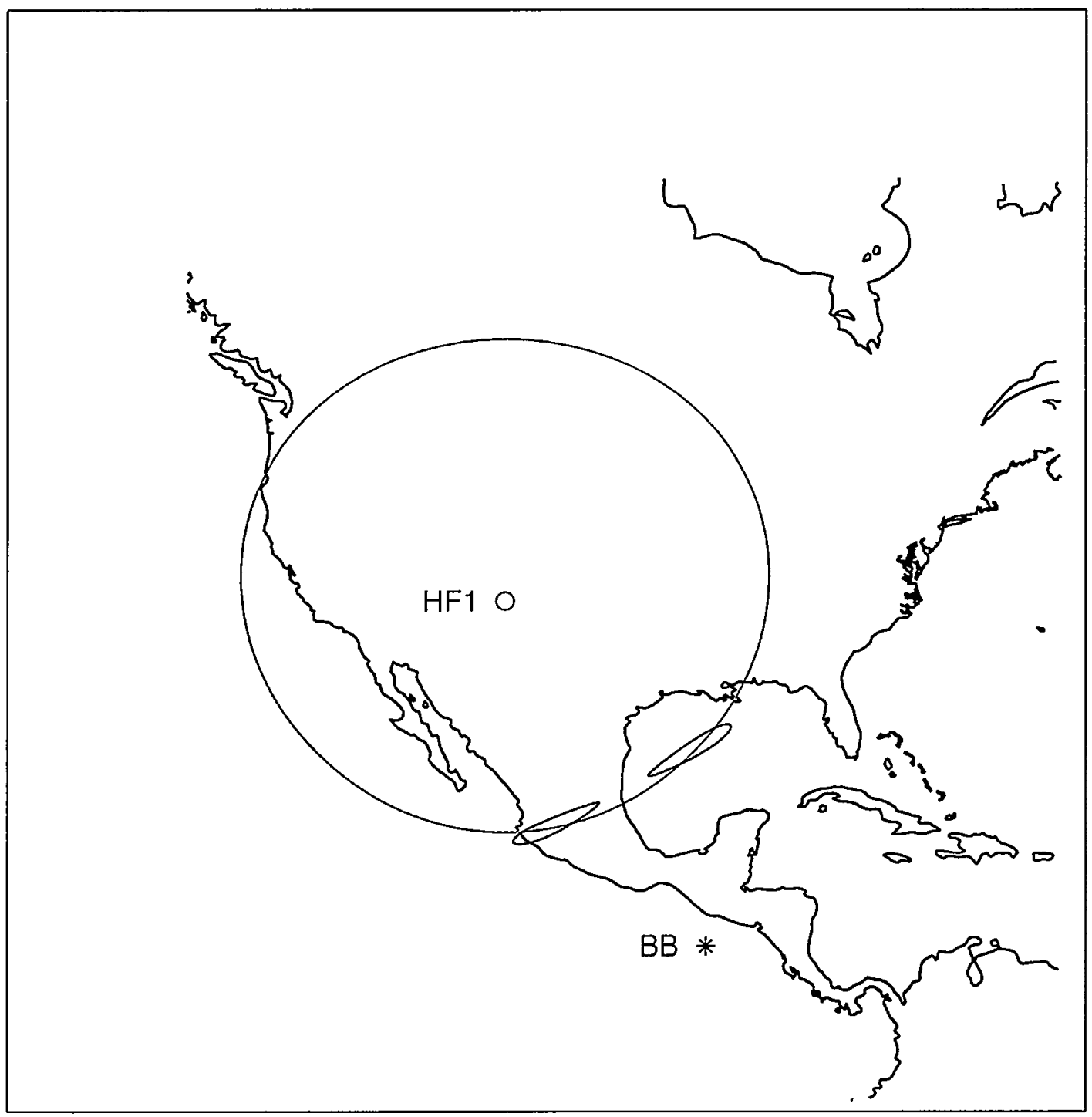

Figure 5.13: Results of location method two, which made use of the estimated range to the source from HF1 and the differential times of arrival between the Blackbeard and HF recordings of the third TIPP event on 11 September 96. The blue airfoils show the most likely source regions. 
uncertainty in Blackbeard timing was approximately $430 \mu$ s. Obviously the Blackbeard timing uncertainty dominates the overall DTOA uncertainty. A program was written to determine the points on the constant radius arc for which the DTOA relationship was satisfied, taking into account the uncertainties in the range and the relative timing measurements. The program output is depicted in Figure 5.13. The two, blue, airfoil-shaped contours are the error ellipses for the two possible source regions. As was the case in the previous method, two possible source locations were obtained. One solution was over Western Mexico and the other was over the Gulf of Mexico. A comparison of the results from this method with those from the previous method is shown in Figure 5.14. The green triangles show the solution from method 1 and the blue airfoils show the solution from method 2. The comparison further suggests that the actual source location was the region over western Mexico, where the two location solutions lie in close proximity to each other and even overlap by a small amount. The locations of the two solutions over the Gulf of Mexico are many hundreds of kilometers apart.

\subsubsection{Data from the National Lightning Detection Network}

The National Lightning Detection Network is a nationwide array of commercial, ground-based lightning receivers. The network provides the locations and times of cloud-to-ground lightning flashes or strokes to its customers. NLDN receiver locations are shown as green circles in Figure 5.15. Stations use magnetic direction finding sensors and electric field change meters to determine source locations and 
Source Location Methods One and Two for TIPPs on Day 96255

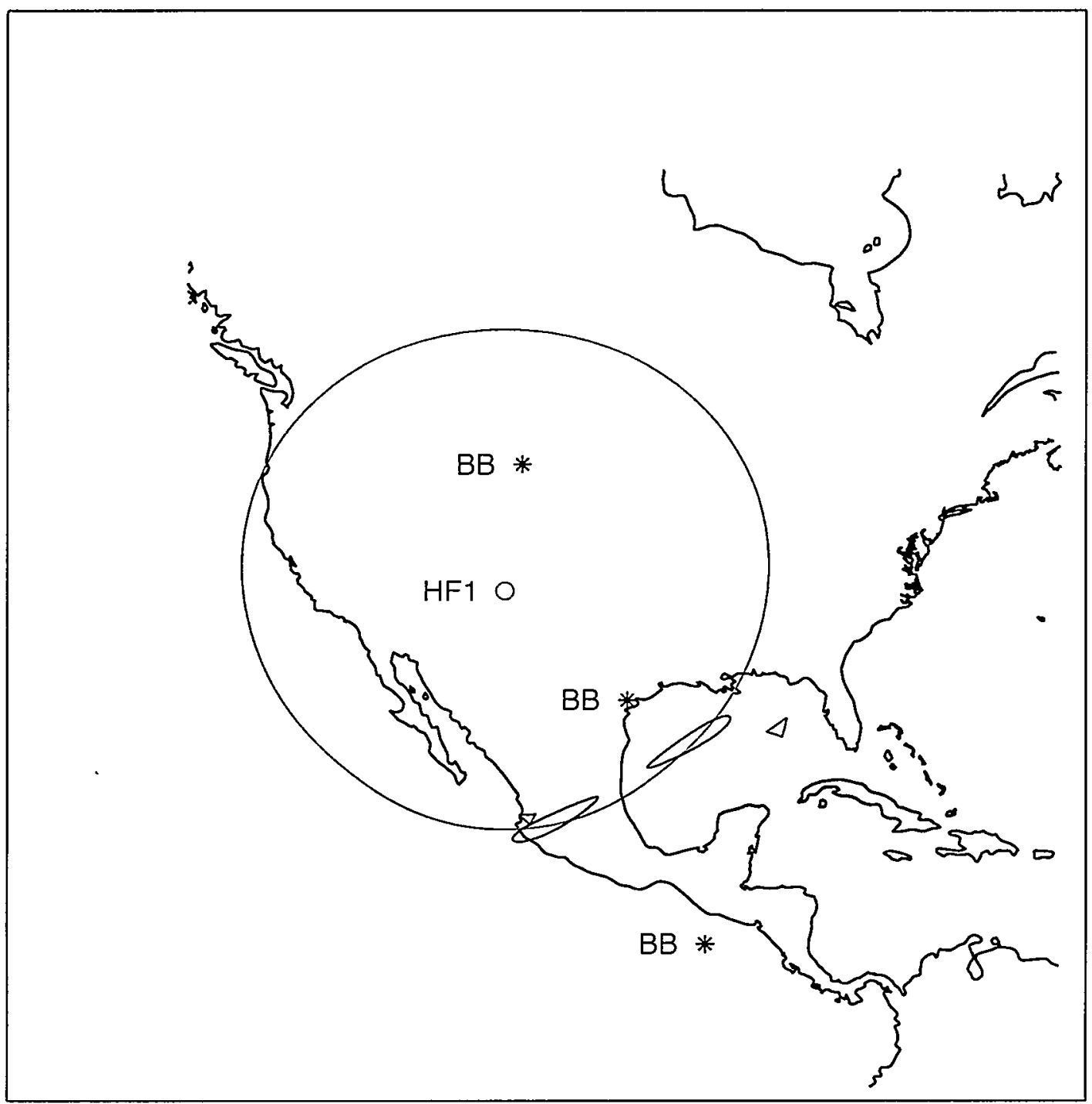

Figure 5.14: Results of location methods one and two for data acquired on 11 September 1996. Method one results are indicated by green triangles. Method two results are indicated by blue airfoils. The close proximity of the westernmost regions, near Punta Eugenia, Mexico, suggests that the events originated in that area. 
NLDN Site Locations and Event Locations on Day 96255

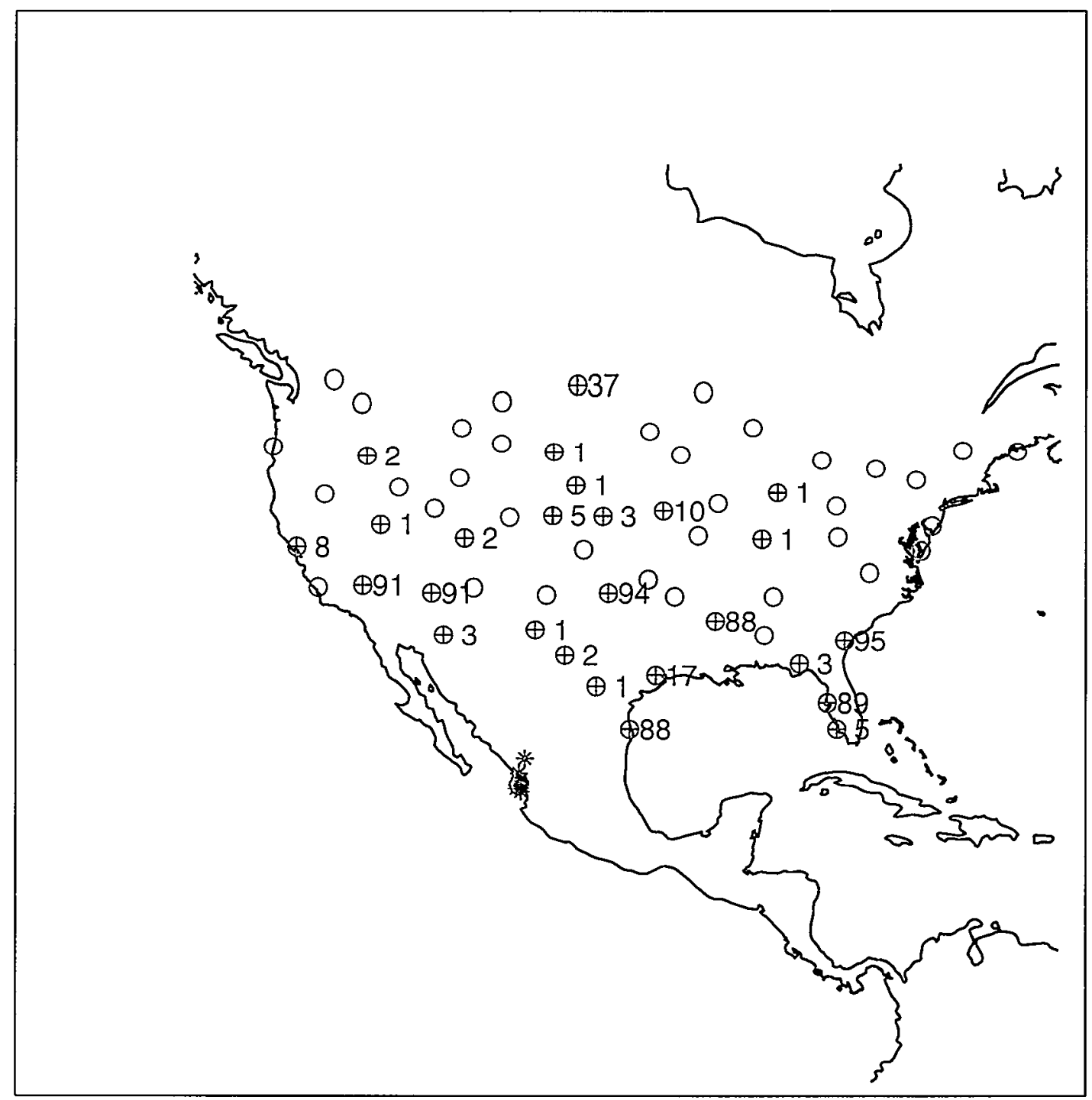

Figure 5.15: Locations of NLDN receivers and locations of events recorded by the NLDN on 11 September 96. NLDN receiver locations are shown as green circles. Stations that recorded signals from CIDs have red plus symbols within their green circles. Numbers next to the station locations indicate the number of events out of 97 that were recorded by the individual stations. CID locations are indicated by magenta asterisks. They are tightly clustered on the western coast of Mexico. 
characterize event waveforms. NLDN ground stroke location uncertainties are on the order of a few kilometers.

The NLDN is specifically designed to report only cloud-to-ground lightning flashes. They developed a number of waveform criteria to distinguish CG flashes from the more common intracloud flashes. Although data from events that do not meet the CG criteria are not reported in the standard final product delivered by NLDN, they are archived on magnetic tape. NLDN stores event times as well as a few specific waveform parameters such as risetime and peak-to-zero time. LANL arranged the purchase of raw, archived NLDN data for the time periods during which the two coincident detections occurred between the ground-based HF station and Blackbeard on 11 and 19 Sep. 1996.

Trigger times of NLDN events from all of the North American stations were compared to the trigger times of the three events recorded by Blackbeard and the 111 events recorded by the LANL HF station on 11 Sep. For 97 of the $111 \mathrm{HF}$ trigger times, between 3 and 11 NLDN stations recorded triggers that were coincident with the HF trigger times. The small timing differences between the HF events and the multi-station NLDN events indicated that the received signals were from the same sources. The large number of coincidences provided evidence that the high event rate seen by Blackbeard and the ground-based HF station was also observed by the NLDN. The NLDN did not report the events in their final product because the events did not meet their criteria for cloud-to-ground lightning strokes. The NLDN would not have reported the events anyway, because the computed source locations would have fallen well outside of the region around the continental United States for which the NLDN 
reports events. Because at least 3 stations recorded times of arrival for the vast majority of the events, it was possible to locate the sources of the events in latitude and longitude based on their differential times of arrival at multiple stations. NLDN stations that recorded coincident events are represented by red plus signs within their green circles in Figure 5.15. Numbers adjacent to the plus signs indicate how many of the events out of 111 were detected by their corresponding stations.

Bob Franz of LANL wrote a geolocation routine to determine the source locations for the events recorded by multiple NLDN stations. The routine involved two steps. In the first step, a rough source location was determined for each event by analyzing the DTOAs between selected NLDN station pairs. In this step, station times of arrival that produced inconsistent results (perhaps due to false alarms or other, independent events that occurred in close temporal proximity to the desired events) were identified, so that they could be eliminated prior to the second step. In the second step, Franz used a least squares routine to find the point on the surface of the earth (in the vicinity of the initial, rough location) that produced the minimal timing error between the delays calculated by the program and the delays measured by the NLDN. The routine used a curved earth model and assumed groundwave propagation paths from the sources to the receivers.

Figure 5.15 shows the locations of the 97 events that were recorded by at least three NLDN stations and occurred in coincidence with LANL HF or Blackbeard event detections on 11 Sep. The events are represented by magenta asterisks and are tightly clustered in a group just north of Punta Eugenia off of the western coast of Mexico. The events are remarkably well clustered considering the fact that most of the 
recording stations were located at distances greater than $1500 \mathrm{~km}$ away from the source.

It is interesting to note the locations of the stations that recorded large numbers of events in relation to the locations of the sources. Most of the stations lie within a band that extends from southern California to Florida. The LANL HF1 station was also within this band. It is the author's belief that these stations were well-situated from the source to receive signals reflected by the ionosphere. The signals received within this band were probably 1-hop reflections from the E-layer, as supported by the fact that the LANL HF1 station was triggered by a 1-hop electric field change signal. The two NLDN stations that recorded quite a few signals and did not lie within this band were the stations in Port Isabel, TX and Minot, ND. These stations were the closest and one of the furthest stations from the sources respectively. The Port Isabel station may have been in a position to receive strong groundwave signals. The Minot station may have been in a position to receive strong 2-hop E-layer signals.

\subsubsection{A Comparison of the Location Techniques}

Figure 5.16 shows a close-up view of the results from location methods 1 and 2 and from the NLDN study. The results from methods 1 and 2 are represented by green triangles and blue airfoils respectively. The locations of the 97 NLDN events are represented by magenta asterisks. Recall that method 1 made use of the HF source height estimate and the pulse separations of the three TIPP events. Method 2 made use of the HF source range estimate and the differential time of arrival between 
Comparison of Methods One and Two and NLDN for Events on Day 96255

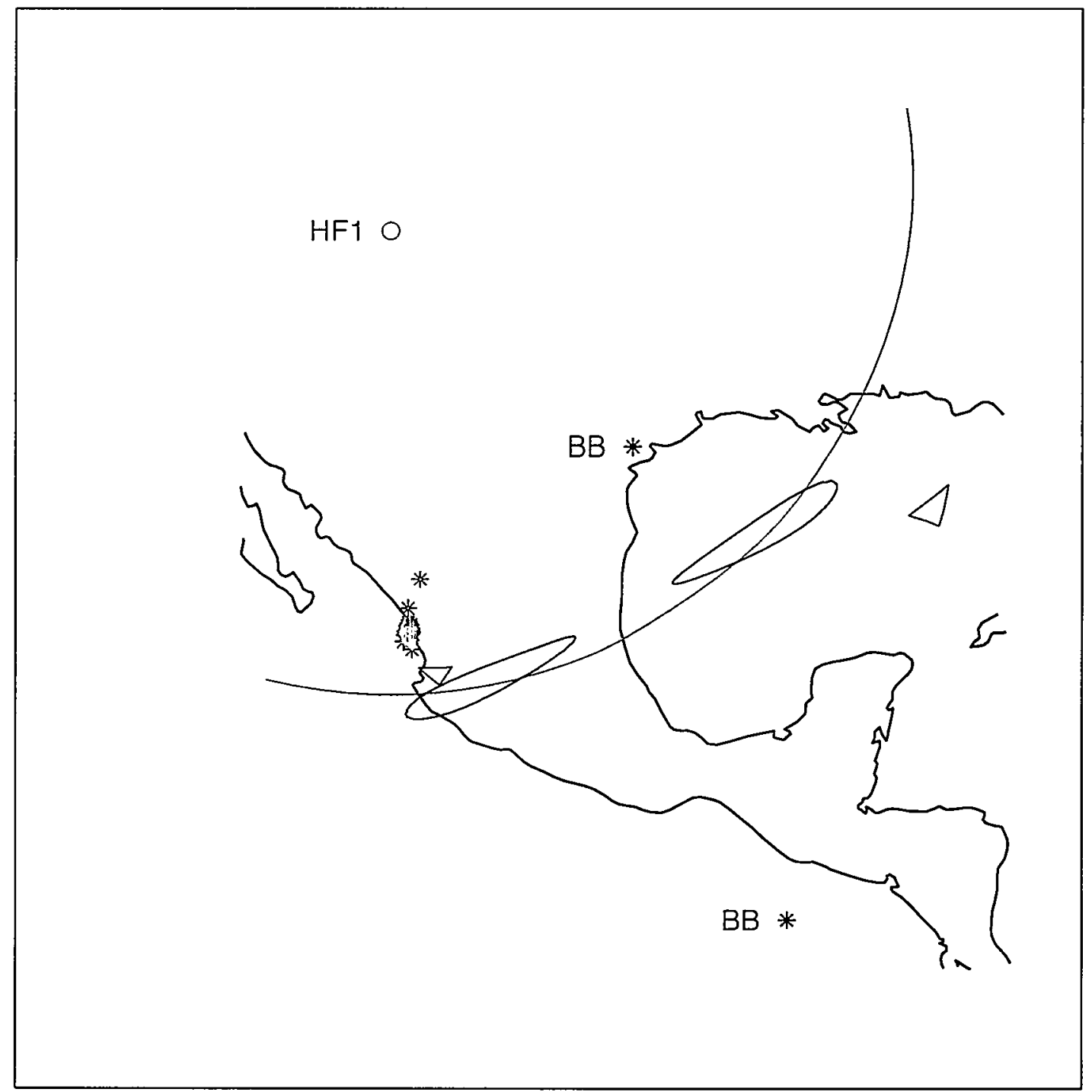

Figure 5.16: Comparison of results from source location methods one and two and from the NLDN data recorded on 11 September 96. The data are consistent with a source region near Punta Eugenia, along the coast of western Mexico. 
the coincident recordings by Blackbeard and HF1. The techniques yield source locations that lie in close proximity to each other along the western coast of Mexico and identify that area as the source region. The small discrepancies between the results are attributed to one or more of the following possible error sources.

In the case of the first location technique, an important assumption was that the sources occurred at the same location and altitude. Although small differences of even tens of $\mathrm{km}$ in the source locations would not have altered the results by a large margin, differences of one or two $\mathrm{km}$ in the source height could shift the location rings significantly. Recall that the spatial difference between the inner and outer rings for each of the TIPP location annuli (in Figure 5.11) resulted from an estimated source height uncertainty of $\pm 2 \mathrm{~km}$.

In the case of the second location technique, the estimate of the range to the source from the LANL HF station was highly dependent on the validity of the assumptions of the ionospheric model. Because the HF paths propagated quite obliquely between the source and receiver, small differences in virtual reflection heights could have led to a significant range errors. Recall that it was assumed that virtual reflection heights were independent of angle of incidence. Although this is usually a good assumption for reflections from the E-layer, the assumption is not as good in application to reflections from the F-layer. The virtual heights for the 1-hop F-layer reflections in Figures 5.2, 5.3, 5.7, and 5.8 could have been different than the virtual height of the F-layer reflection for the 2-hop E-F reflections. The difference could have led to a range estimate value that was too high. 
In the case of the third location technique, which made use of the independent NLDN data set, it is believed that the source locations were quite accurate. The fact that more than three stations (and as many as 11 stations) recorded and time tagged most of the events added redundancy to the location technique and made it possible to determine timing biases between the stations and to disregard inconsistent data. The fact that the event locations were tightly clustered (90\% of the events fell within a 20 $\mathrm{km}$ radius of the centroid location) indicates that random errors were quite small. There is one assumption that was made using this technique that is subject to some question, however. This was the assumption that all stations recorded groundwave signals from the source. Beyond a distance that is usually between 500 and $1000 \mathrm{~km}$, E-layer reflections of field change events produce larger-amplitude signals than the groundwave signals. It is likely that many of the NLDN stations triggered off of the one-hop E-layer reflections (or even two-hop reflections in the case of the distant detections), rather than off of the groundwave signals from the sources. Note that the LANL HF station received all of its triggers from 1-hop E reflections. Even if this was the case, the event locations probably would not have differed by much, since the path differences are not that great for the very oblique propagation paths and since it is the differential time relationships, rather than the absolute ones, that are important for geolocation.

Despite the small discrepancies between the novel source location methods, the methods provided a clear and consistent pointer to the general source region. 


\subsubsection{GOES-8 Infrared Imagery}

The good agreement between the three location methods leaves little doubt that the source region was along the western coast of Mexico near Punta Eugenia. Figure 5.17 shows an infrared satellite image from GOES-8. The image was acquired 10 minutes after the final Blackbeard arm time on 11 Sep., the arm time during which the coincident detection was made. The image shows that the events occurred in close spatial proximity to a rainband that extended far to the north-northeast from a tropical

cyclone named Fausto. The storm was centered $300 \mathrm{~km}$ from the west coast of Mexico, but a significant buildup of low-temperature cloud tops was present in the exact region pinpointed by the location techniques presented here. The lowtemperature tops in the image are indicative of high clouds possibly formed by vigorous convective activity. High cloud tops are often indicative of thunderstorm electrical activity.

\subsection{SECOND COINCIDENT EVENT: 19 SEPTEMBER 1996}

The second coincident event was recorded by the HF ground station in Los Alamos and the Blackbeard receiver on 19 September 1996. Techniques and analyses similar to those described in the previous sections were utilized to determine the location of the source of the event. During the satellite pass on that date, Blackbeard was armed for three one-minute time periods that began five minutes apart. 


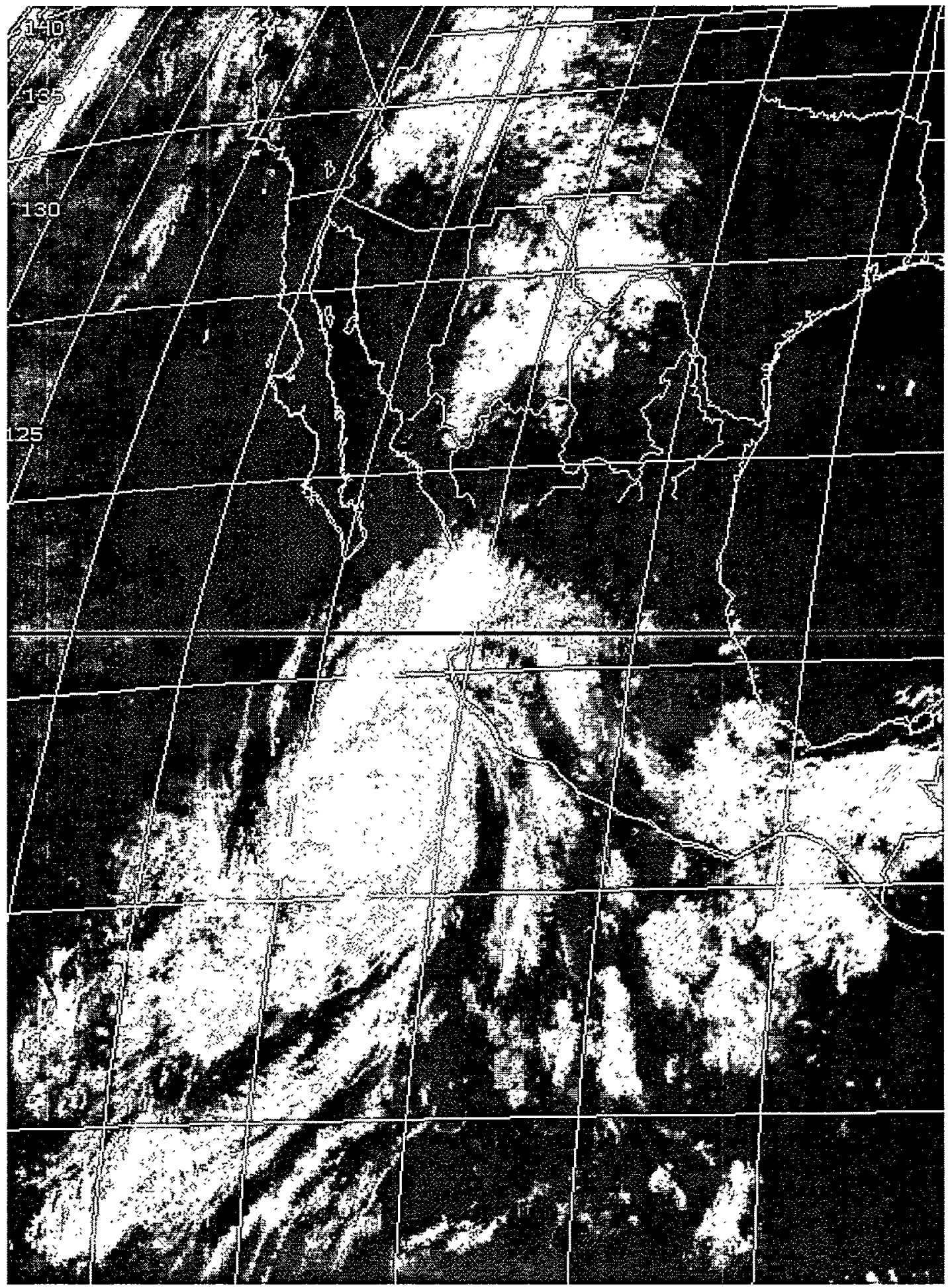

Figure 5.17: Infrared satellite image from GOES-8. The image was acquired ten minutes after the final Blackbeard arm time on 11 September 1996. The image shows that the CID source region occurred in close spatial proximity to cold clouds associated with tropical cyclone Fausto. 
Blackbeard detected and recorded TIPP events during the second and third arm periods, but not the first. Spectrograms of the two events are shown in Figures 5.18 and 5.19. The pulse separations for the two TIPPs were 40 and $23 \mu$ s respectively. The Blackbeard sub-satellite points and fields of view at the times of the two triggers are shown in Figure 5.20. Blackbeard was on a descending node, traveling from northwest to southeast, as indicated by the approximate trigger times in the figure. The TIPP event that was coincident with the ground-based HF event was the first of the two detections.

The LANL HF ground station was armed for 14 minutes inclusive of the three Blackbeard arm times. During the 14-minute arm, the ground station recorded four very powerful events with matching time-frequency spectrograms. The events originated over the horizon as indicated by their time-frequency characteristics. The event that was coincident with the Blackbeard TIPP event was the third of the four events recorded. Its spectrogram is shown in Figure 5.21. A whitened version of the spectrogram is shown in Figure 5.22. The fourth of the four HF events occurred approximately 5.5 seconds after the third and had a better signal to noise ratio. Because it better illustrates the signal time-frequency characteristics, it is pictured in Figure 5.23. Its whitened version is shown in Figure 5.24.

Figure 5.25 shows a time relationship plot similar to that generated for the analysis of the 11 Sep. data, but for the 19 Sep. data. As was the case in the previous analysis, Blackbeard triggers occurred near the beginnings of the arm windows of the two successful arm periods, at approximately 10 and 1 seconds respectively. The 


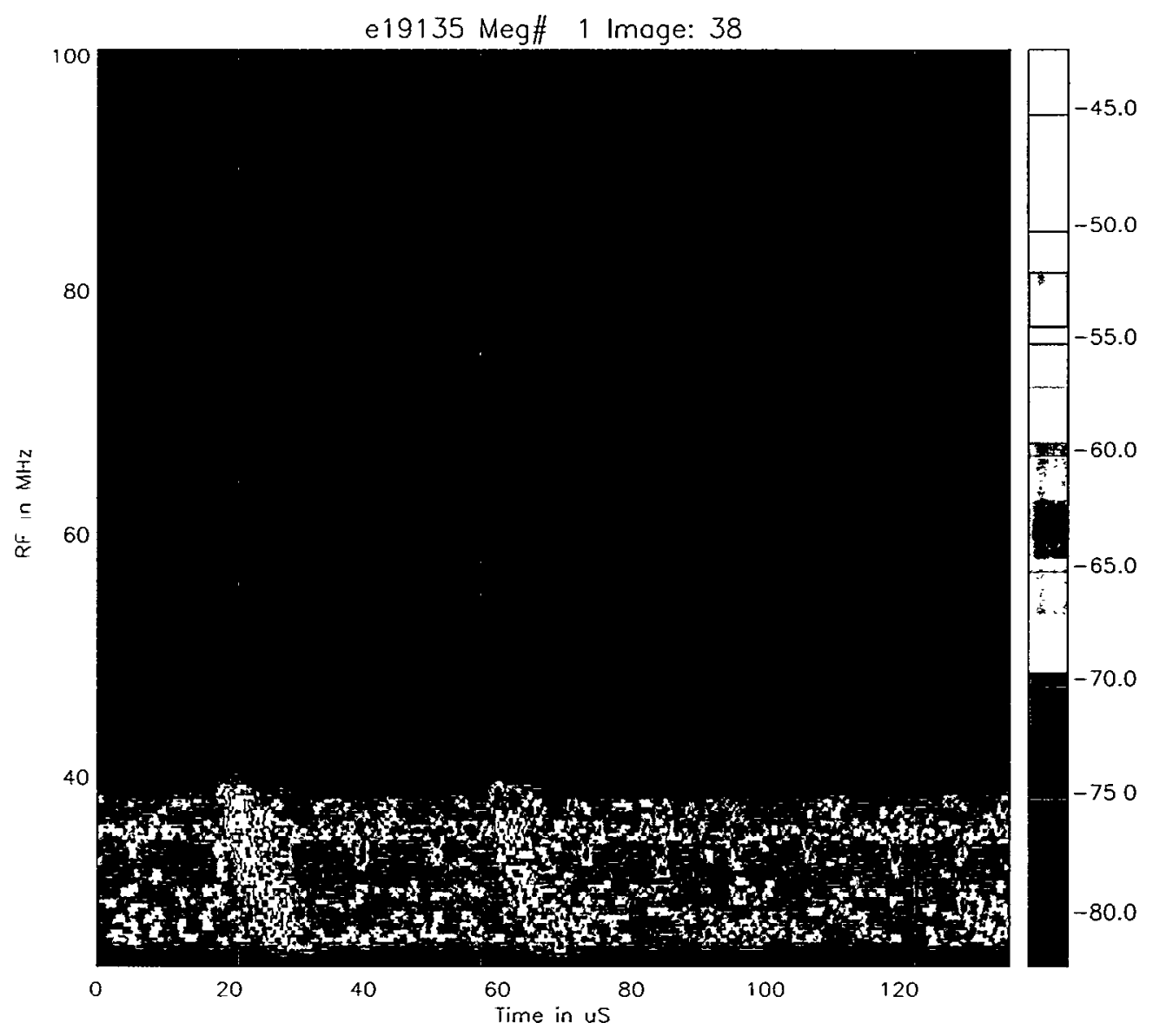

Figure 5.18: Spectrogram of the first TIPP event recorded by Blackbeard on 19 September 96 . The event was also recorded by the LANL broadband HF station (see Figure 5.21). 


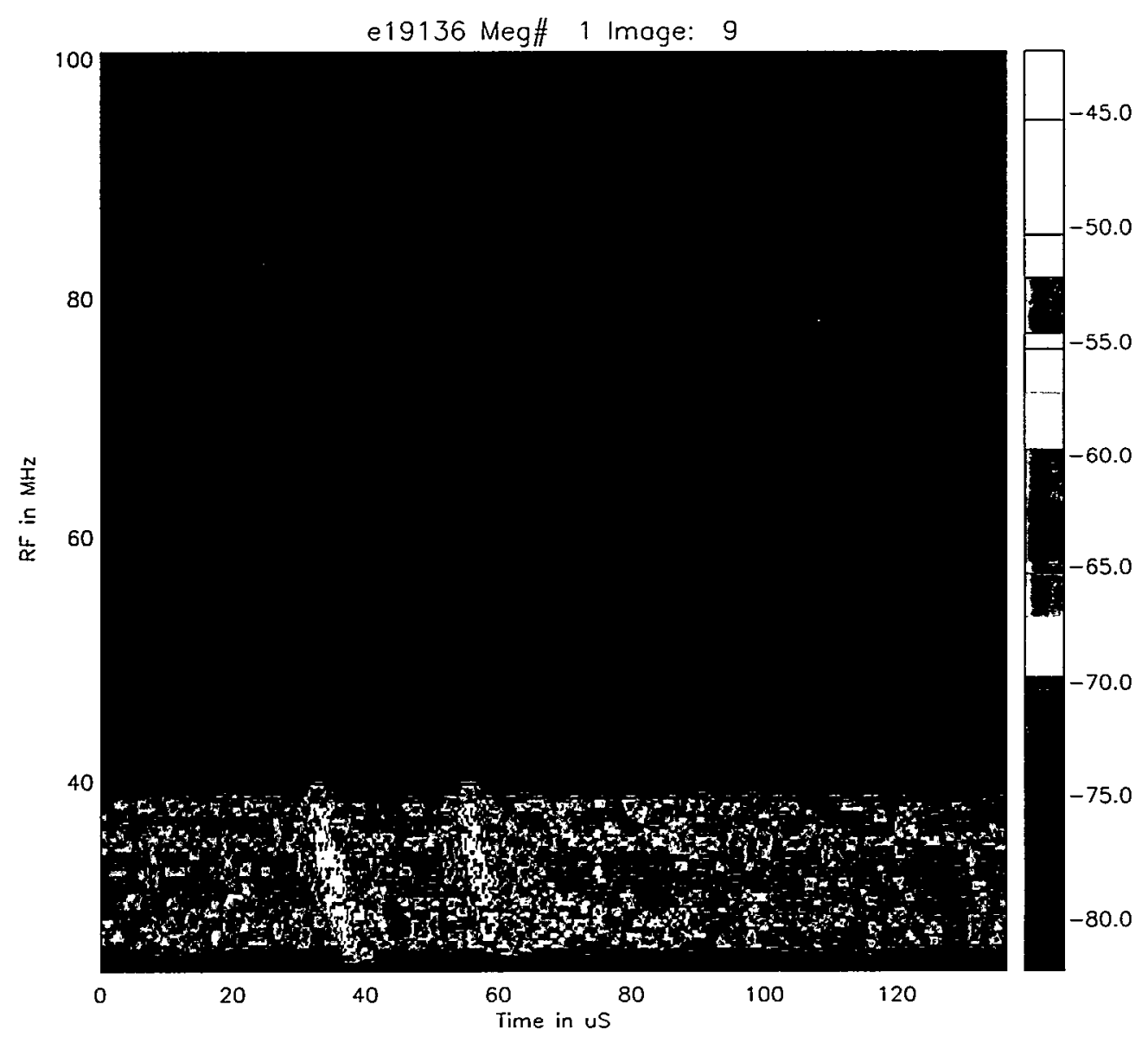

Figure 5.19: Spectrogram of the second TIPP event recorded by Blackbeard on 19 September 96. 
Blackbeard Sub-Satellite Points and FOVs for TIPPs on Day 96263

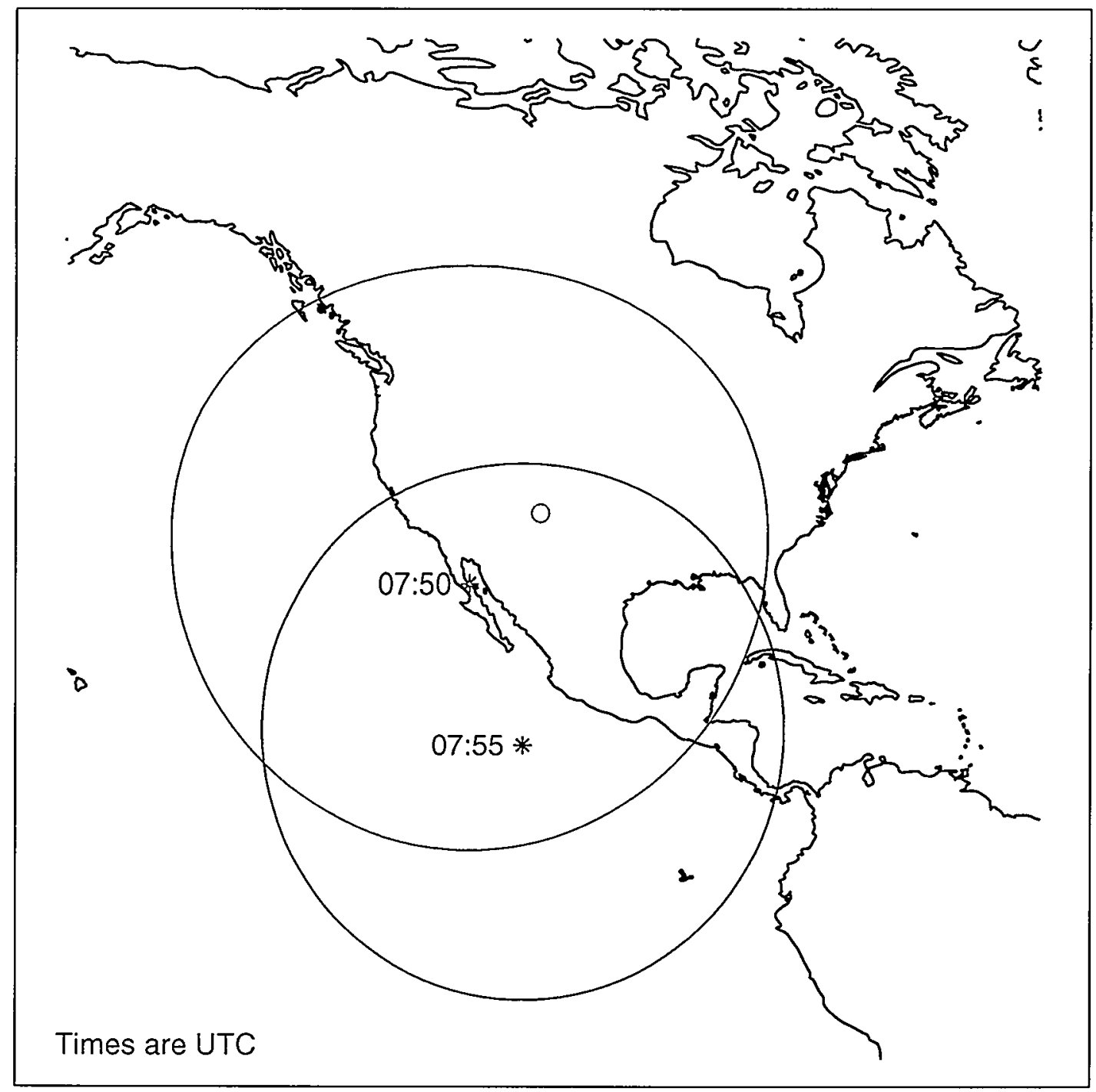

Figure 5.20: Blackbeard sub-satellite locations and fields of view during the times when the two TIPP events were recorded on 19 September 96. The sub-satellite points are represented by colored asterisks. The correspondingly-colored lines show the location of the optical horizon as viewed from Blackbeard. 
HF:x62b134: UTC: 1996263 07:50:10.157451, Range $=1229 \mathrm{~km}$

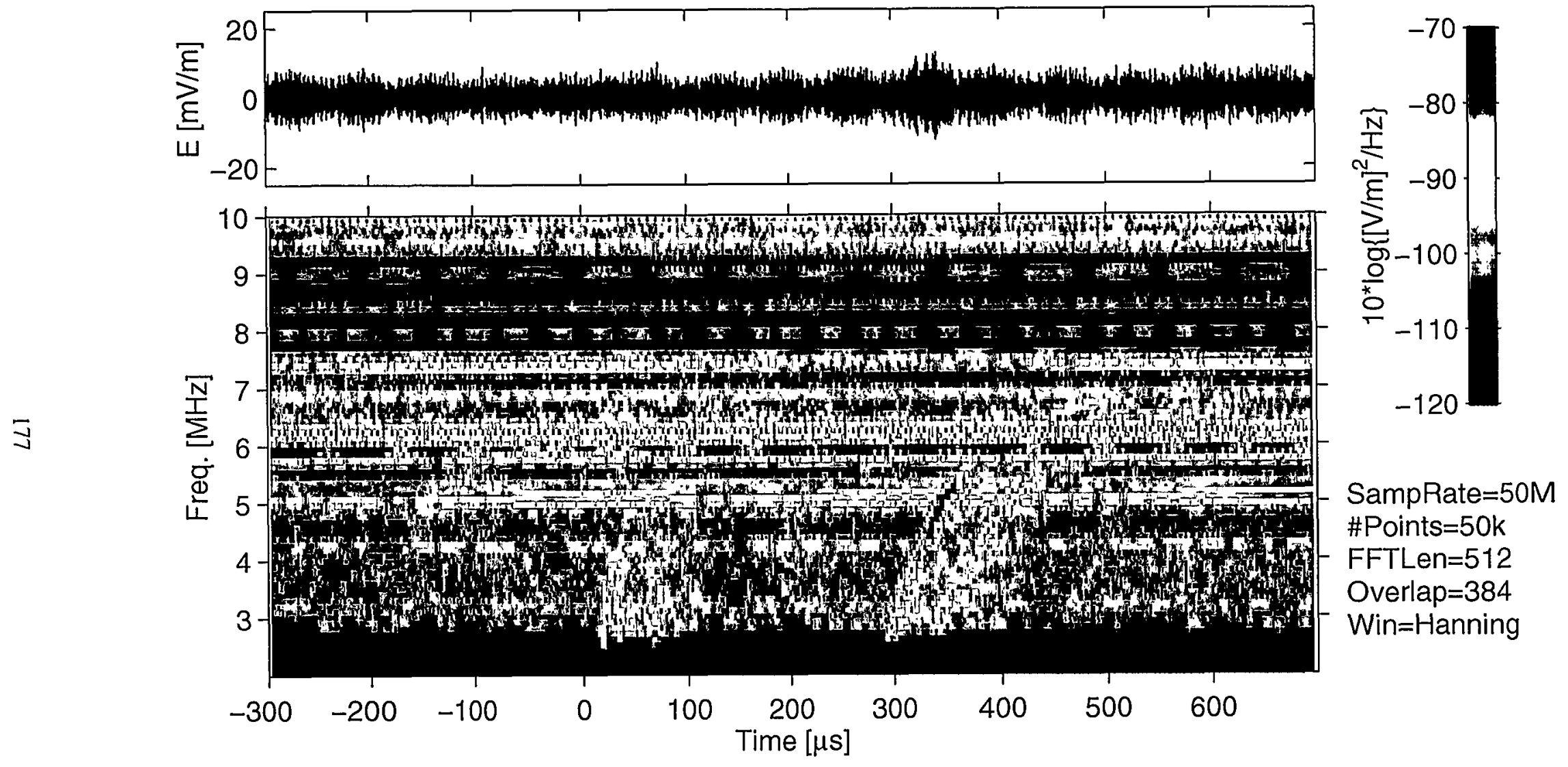

Figure 5.21: HF electric field waveform and spectrogram from a CID recorded on 19 September 1996. Signals from multiple layers of the ionosphere were received. The event was coincident with the Blackbeard TIPP event pictured in Figure 5.18. 


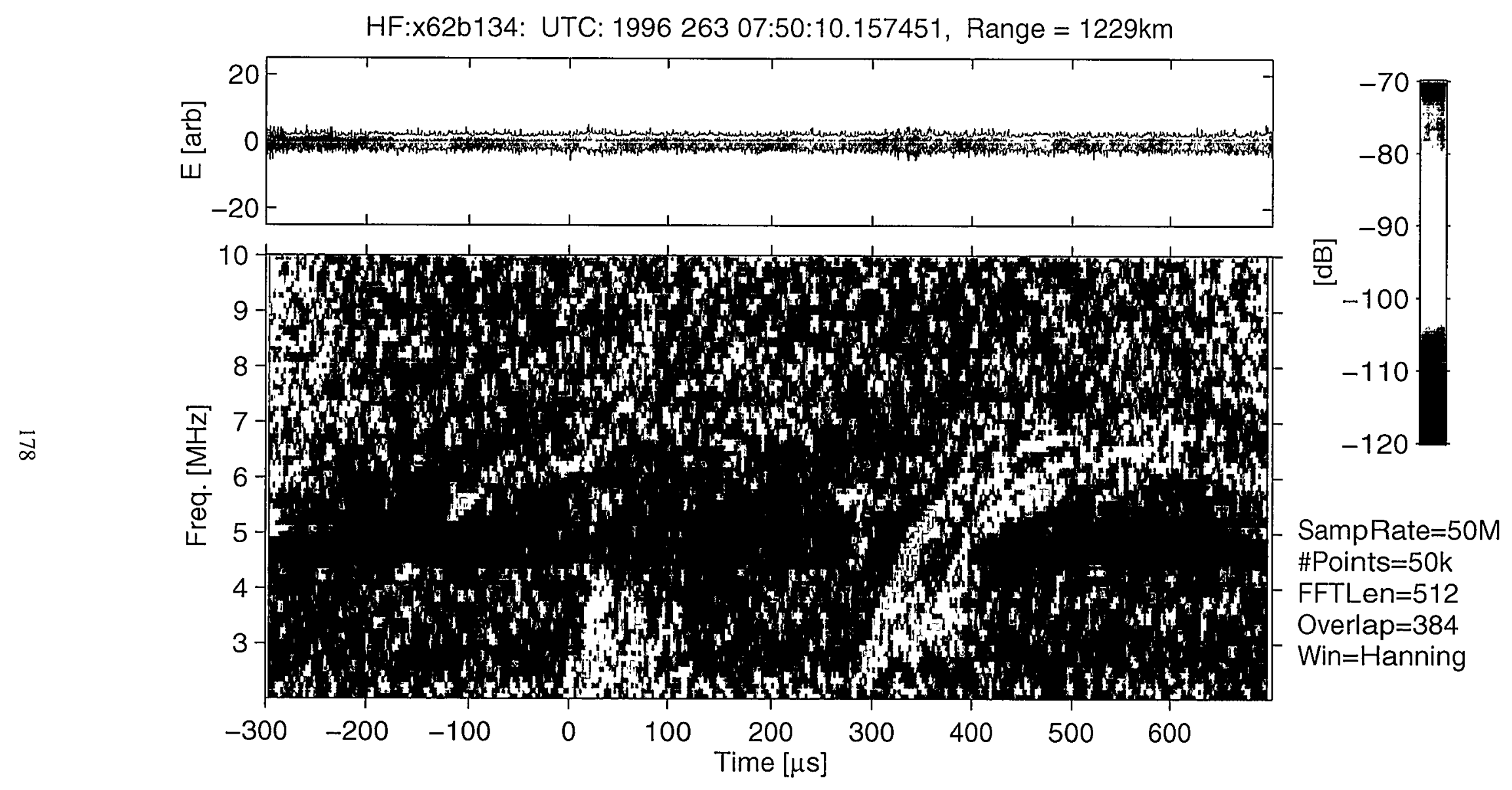

Figure 5.22: "Whitened" version of the waveform and spectrogram from Figure 5.21. 


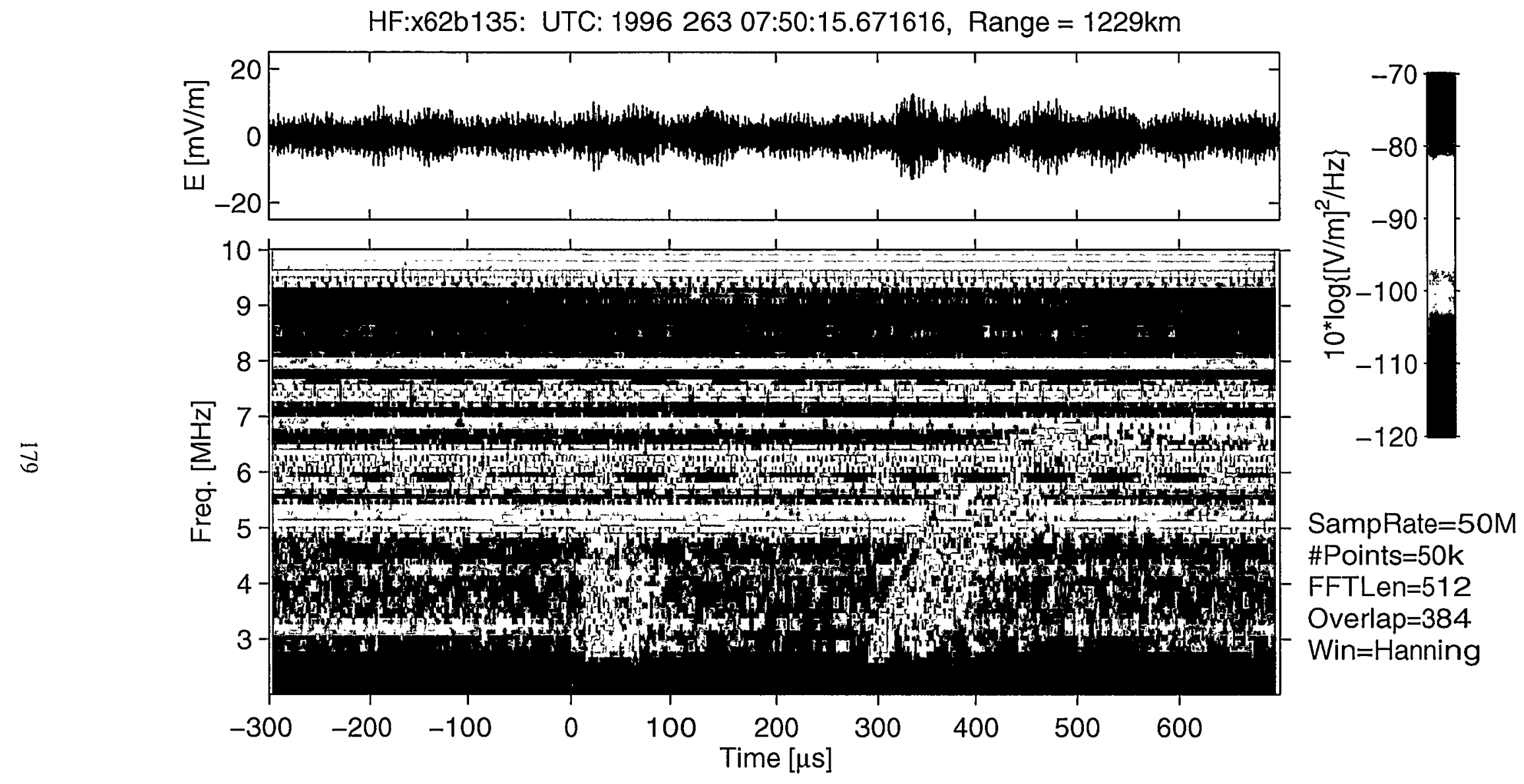

Figure 5.23: HF electric field waveform and spectrogram from a CID recorded on 19 September 1996. The event had a better signal-to-noise ratio than the event pictured in Figure 5.21. 


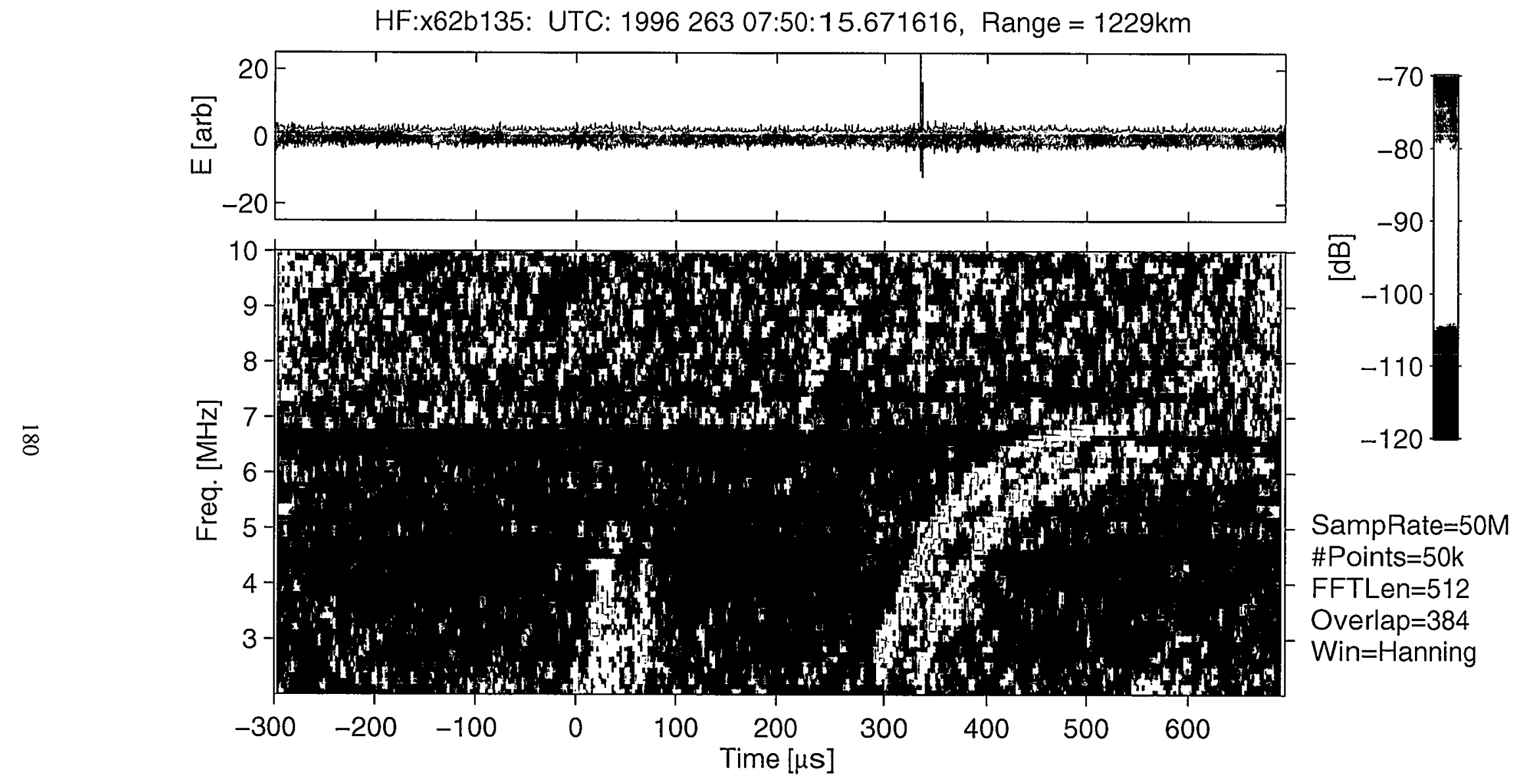

Figure 5.24: "Whitened" version of the waveform and spectrogram from Figure 5.23. 


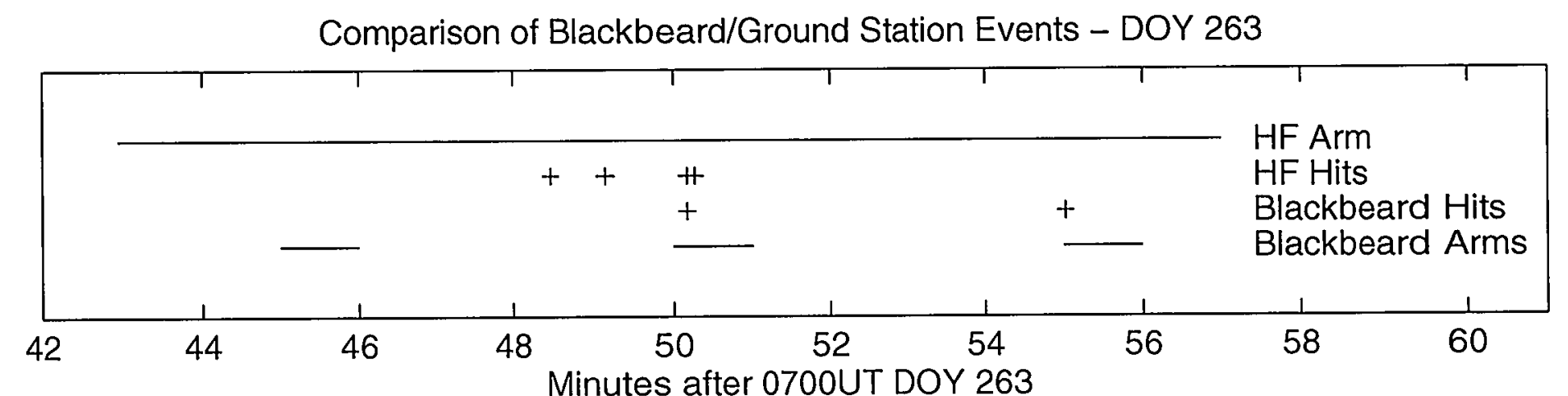

First Event

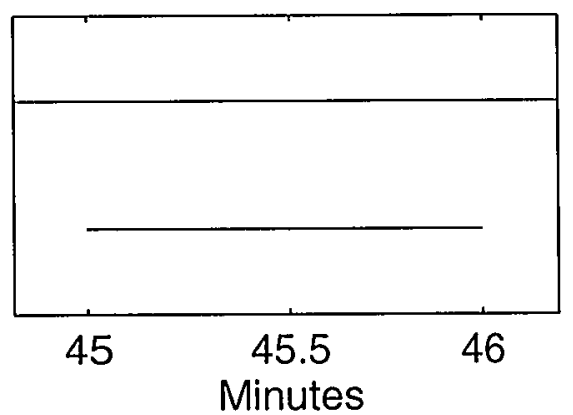

Second Event - BINGO

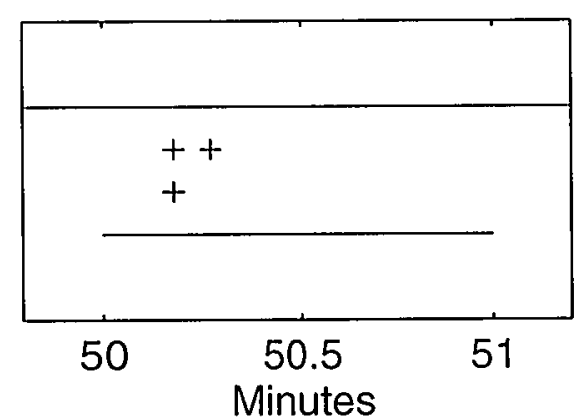

Third Event

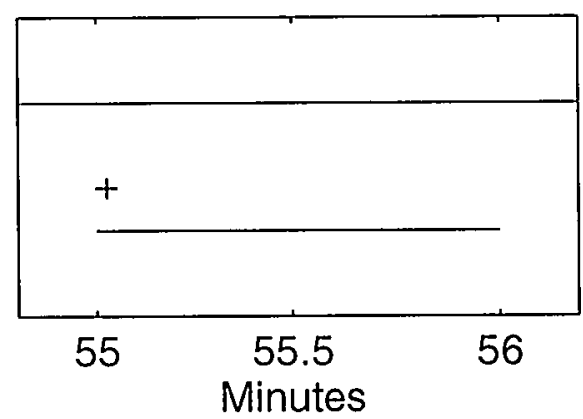

Figure 5.25: Time sequence of events on 19 September 1996. The plots show the arm periods and trigger times for Blackbeard and the ground-based HF system. The coincident event occurred during the second Blackbeard arm period. 
short arm-to-trigger times are suggestive of a high trigger rate from the TIPP source region. No triggers were received during the first Blackbeard arm period.

The HF spectrograms in Figures 5.21 through 5.24 are simpler to interpret than the ones for the previous case. Each spectrogram features two pairs of distinct events. The first pair of pulses appears undispersed. The pulses occur at 20 and $36 \mu$ s in the figures. The first pulse is the 1-hop E-layer reflection of the signal from the ionosphere. The second pulse is the 1-hop E-layer reflection of the ground reflection of the source. These two propagation paths were illustrated in the first panel of Figure 5.10. The second pair of pulses is dispersed. The pulses occur at 349 and 391 $\mu \mathrm{s}$ in the figures (at a frequency of $5 \mathrm{MHz}$, the frequency used for the analysis). The pulses are 1-hop reflections from the F-layer, the second being the ground-reflected pulse. These two propagation paths were illustrated in the third panel of Figure 5.10. Unlike the HF spectrograms shown in the previous analysis, the spectrograms shown here show no signs of multiple-hop signals.

Because fewer reflections were received than for the previous case, it was not possible to solve for all four of the unknowns: source height, E-layer virtual height, $\mathrm{F}$ layer virtual height, and range to the source. So for this case, it was assumed that the virtual height of the E-layer was known. On multiple nights during the summer of 1996 it was observed, based on relative delays of signals reflected from the ionosphere, that the virtual height of the E-layer remained fairly constant for obliquely propagated HF signals. The median value for this height was $97 \mathrm{~km}$.

Under the assumption that the virtual height of the E-layer $(H E)$ was fixed at 97 $\mathrm{km}$, an ionospheric raytracing code similar to that described in Section 5.1.2 was 
developed to find the values of source height $(h)$, source range $(r)$, and F-Layer virtual reflection height $(H F)$ for which the model best matched the data. For this model a flat-earth approximation was utilized to simplify and speed up the program. The effects of the simplification were determined to be fairly small, but not negligible. A range error as large as $50 \mathrm{~km}$ was predicted. The minimum mean square propagation delay error occurred for $h=15.4 \mathrm{~km}, r=1230 \mathrm{~km}$, and $H F=275.5 \mathrm{~km}$.

Using methods similar to the ones used to locate the first coincident event, Blackbeard data were incorporated to estimate the location of the source. Taking into account the calculated source height and the pulse separations for the two TIPPs recorded by Blackbeard (method one from the previous analysis), possible source location annuli were formed. They are shown in Figure 5.26. The two overlap regions between the annuli are depicted by large green diamonds in Figure 5.27. An uncertainty of $\pm 2 \mathrm{~km}$ was applied to the source height calculation and an uncertainty of $\pm 1 \mu$ s to the TIPP pulse separations. Using the calculated source range and the difference in the signal times of arrival at $\mathrm{HF} 1$ and Blackbeard (method two from the previous analysis), two possible source locations were determined. The locations are depicted as blue airfoils in Figure 5.28. An uncertainty of $\pm 100 \mathrm{~km}$ was applied to the source range calculation and an uncertainty of $\pm 860 \mu$ s to the DTOA measurement. The results from both of the location methods are shown in Figure 5.29. The close proximity of the regions in northern Mexico and southern Texas suggest that the actual source region was in that area. 
Source Location Method One for TIPPs on Day 96263

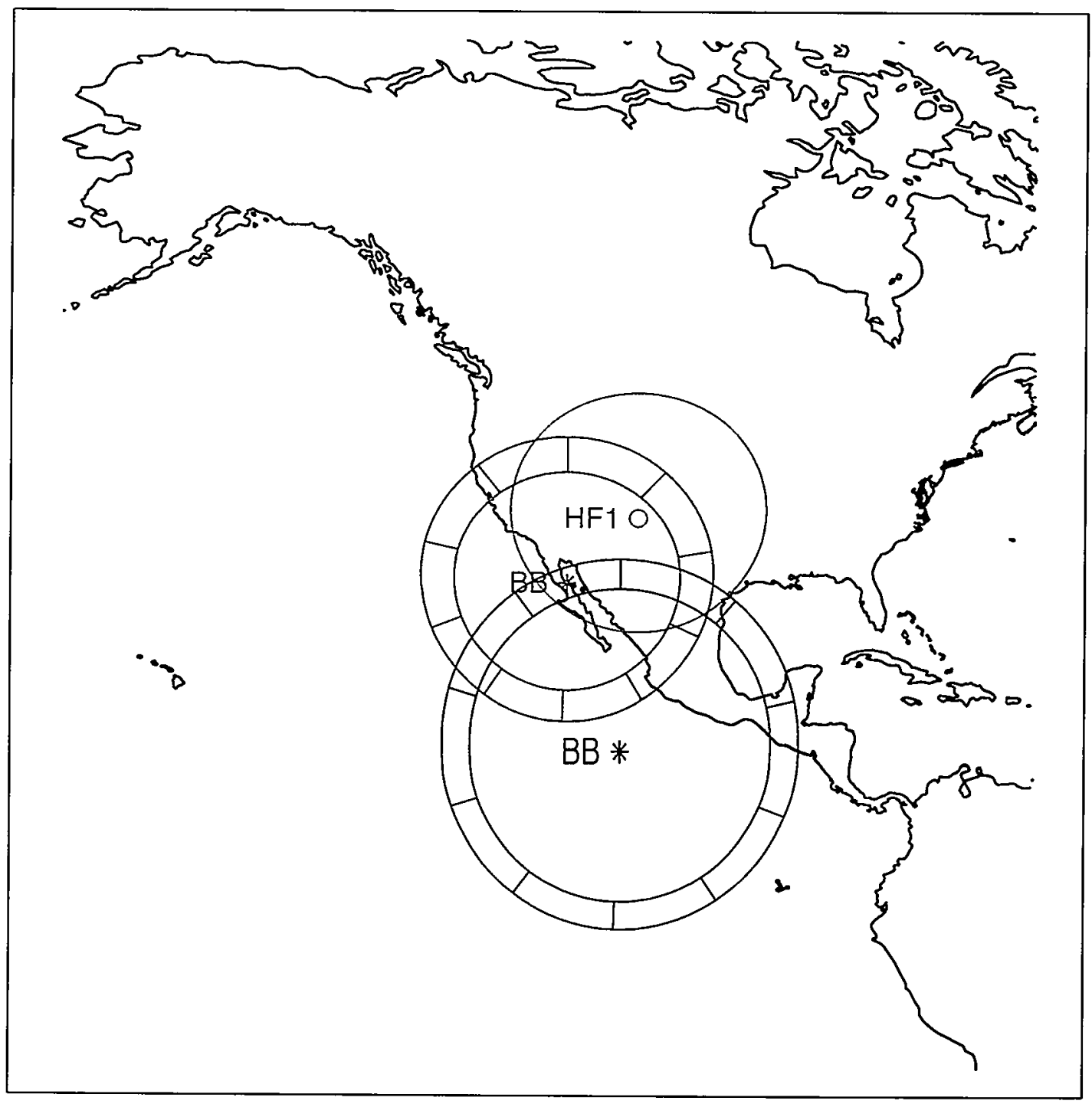

Figure 5.26: Illustration of source location method one for events recorded on 19 September 96. Colored (green, and blue) annuli show the most probable source locations for the two events based on the estimated source height and the pulse separations for the two TIPP events. The cyan circle shows the estimated range to the source from the HFl recording station. 
Source Location Method One for TIPPs on Day 96263

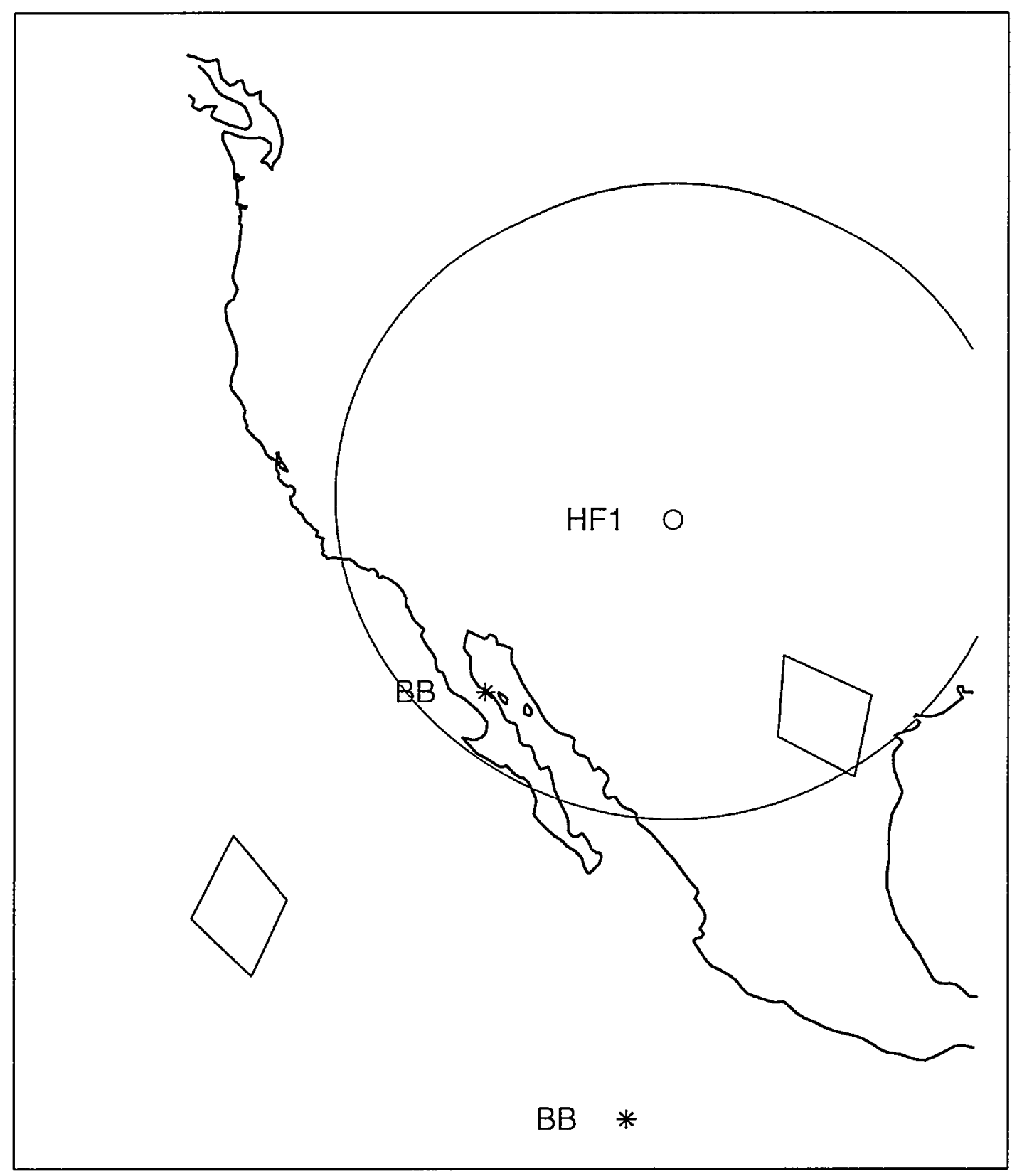

Figure 5.27: Illustration of the most probable source locations based on location method one. The green quadrilaterals show the overlapping portions of the two colored annuli from Figure 5.26. The close proximity of the cyan range circle to the easternmost quadrilateral suggests that the events originated in the vicinity of the quadrilateral. 
Source Location Method Two for TIPP on Day 96263

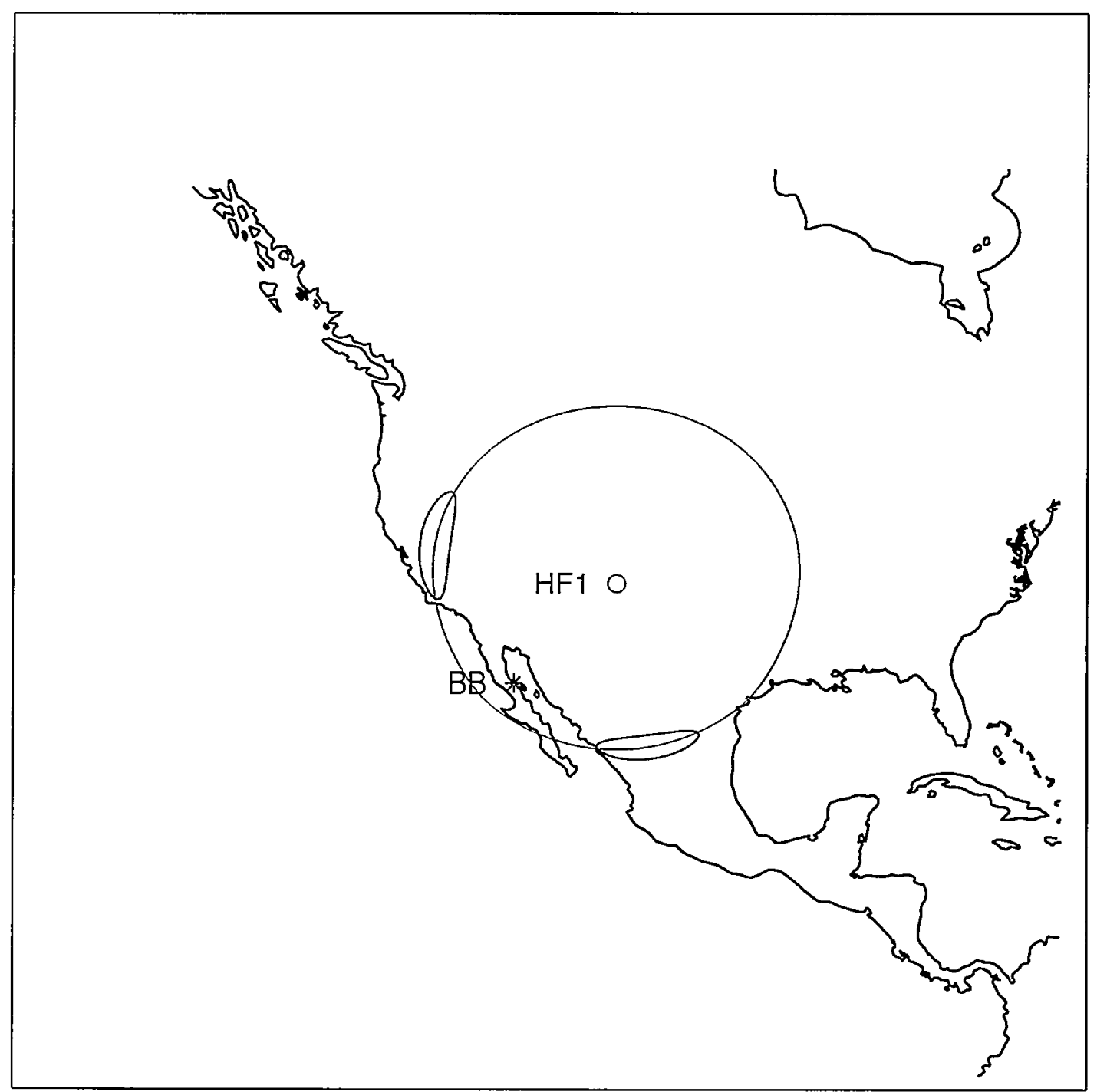

Figure 5.28: Results of location method two, which made use of the estimated range to the source from $\mathrm{HFl}$ and the differential times of arrival between the Blackbeard and HF recordings of the first TIPP event on 19 September 96. The blue airfoils show the most likely source regions. 
Source Location Methods One and Two for TIPPs on Day 96263

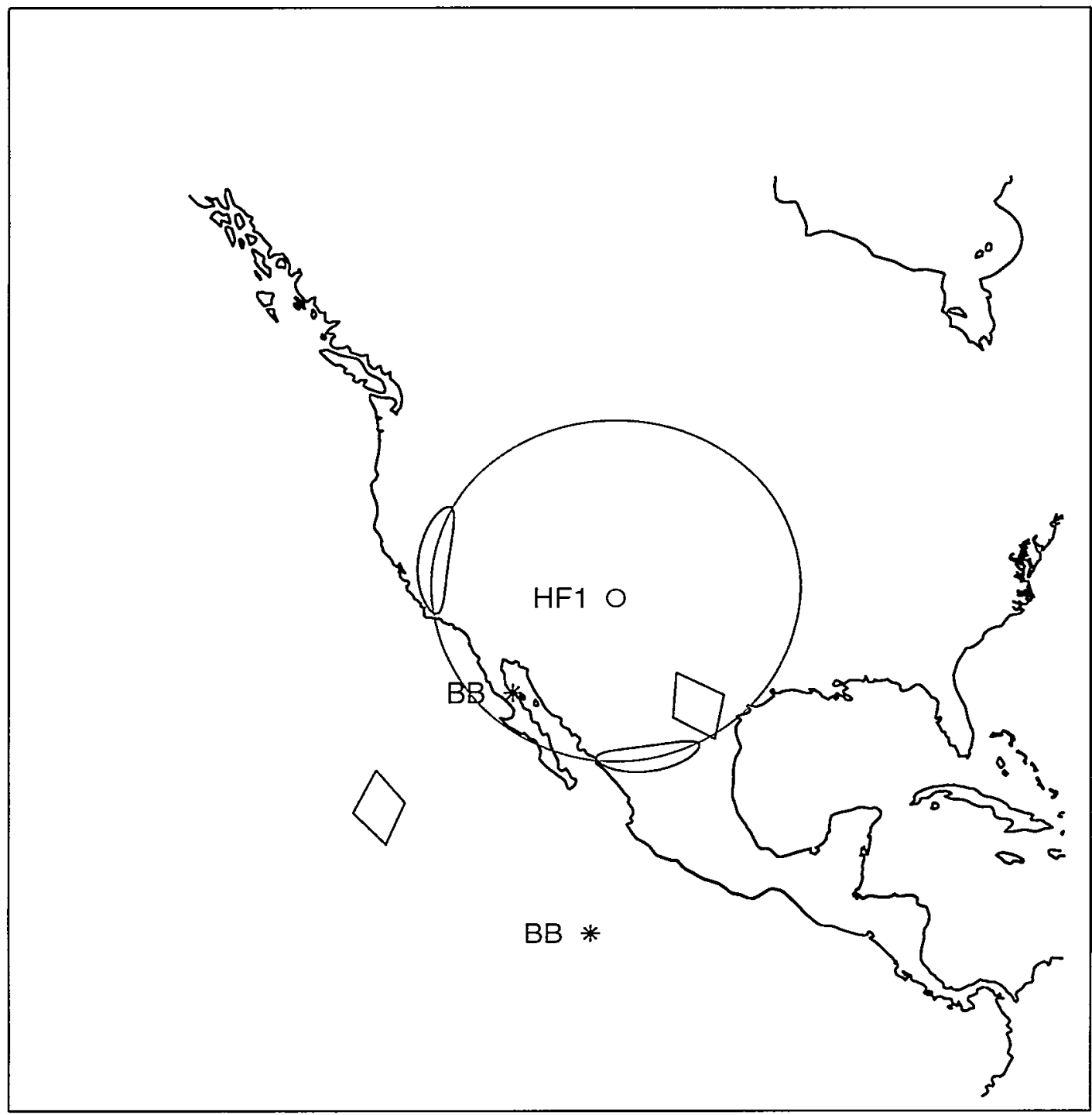

Figure 5.29: Results of location methods one and two for data acquired on 19 September 1996. Method one results are indicated by green quadrilaterals. Method two results are indicated by blue airfoils. The close proximity of the regions in Texas and Mexico suggests that the event sources occurred in that area. 
The National Lightning Detection Network also received signals that were coincident with the four HF triggers on 19 Sep. A program similar to that described in the previous section was written to determine the source locations based on the NLDN data. The results are shown in Figure 5.30 with the results from the other two location methods. The four NLDN locations are represented by magenta asterisks. The data show that south Texas was, in fact, the source region and that the HF/Blackbeard location results were consistent with the NLDN data.

Figure 5.31 shows an infrared satellite image from GOES-8. The image was acquired at the time of the beginning of the first Blackbeard arm period on 19 Sep. Once again the source region appears to have occurred in close conjunction with very high cloud tops. The system that produced the events was actually the remains of tropical cyclone Fausto, the tropical cyclone that produced the emissions described in Section 5.1 .

\subsection{OVF:RVIFW (OF COINCIDENT (GROUND ANI) SATFLLITE OBSFRVATIONS}

The events described in this chapter triggered the Blackbeard receiver, triggered the LANL field change system, were recorded by the LANL HF system, and triggered multiple NLDN lightning location sensors. Based on the data from the various recording platforms, the sources of the events have the following characteristics. Foremost, they produce very powerful radio frequency radiation, as evidenced by the Blackbeard VHF data and ground-based HF data. It is known from other Blackbeard 
Comparison of Methods One and Two and NLDN for Events on Day 96263

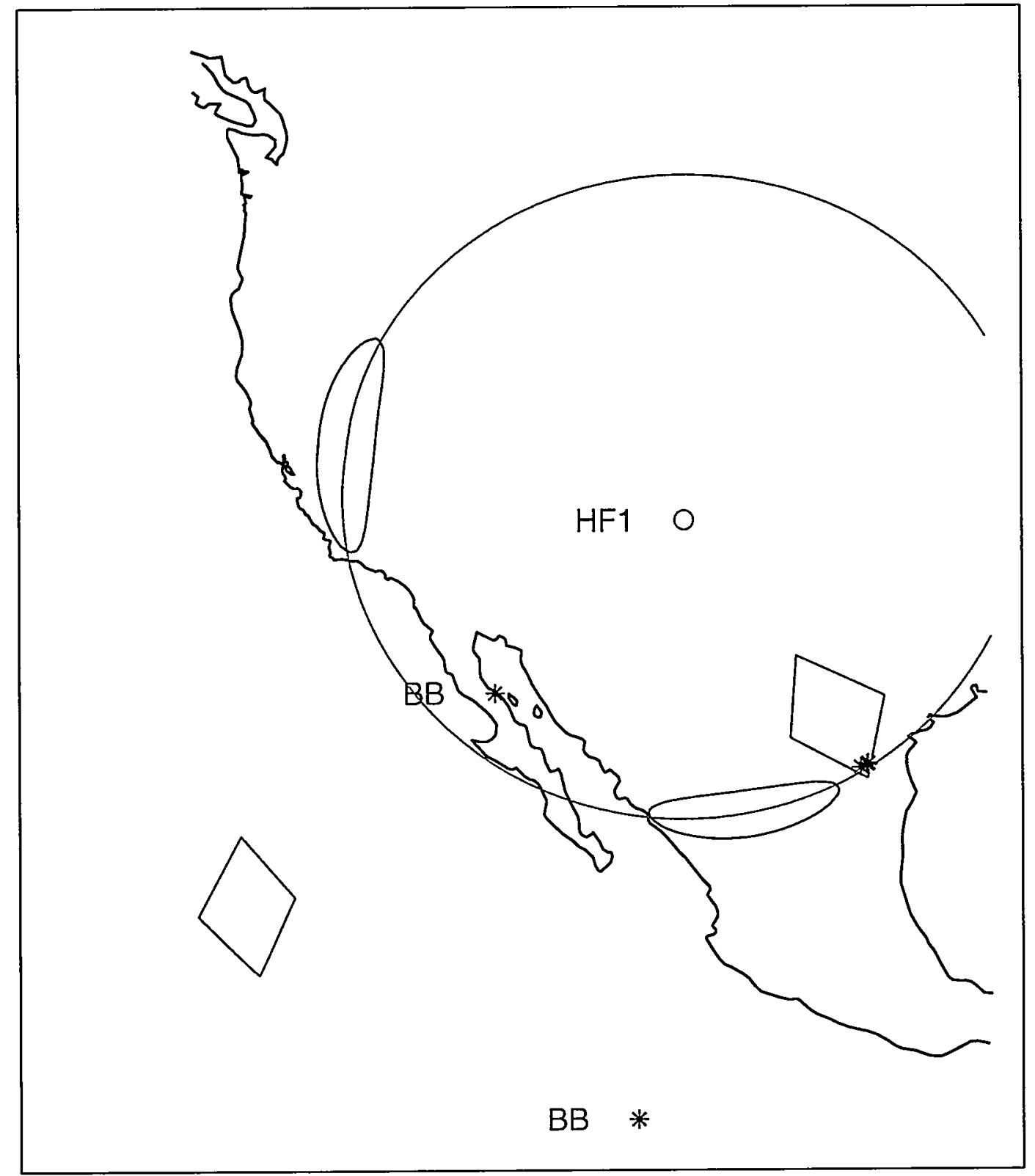

Figure 5.30: Comparison of results from source location methods one and two and from the NLDN data recorded on 19 September 96. The data are consistent with a source region in south Texas, close to the Mexican border. 


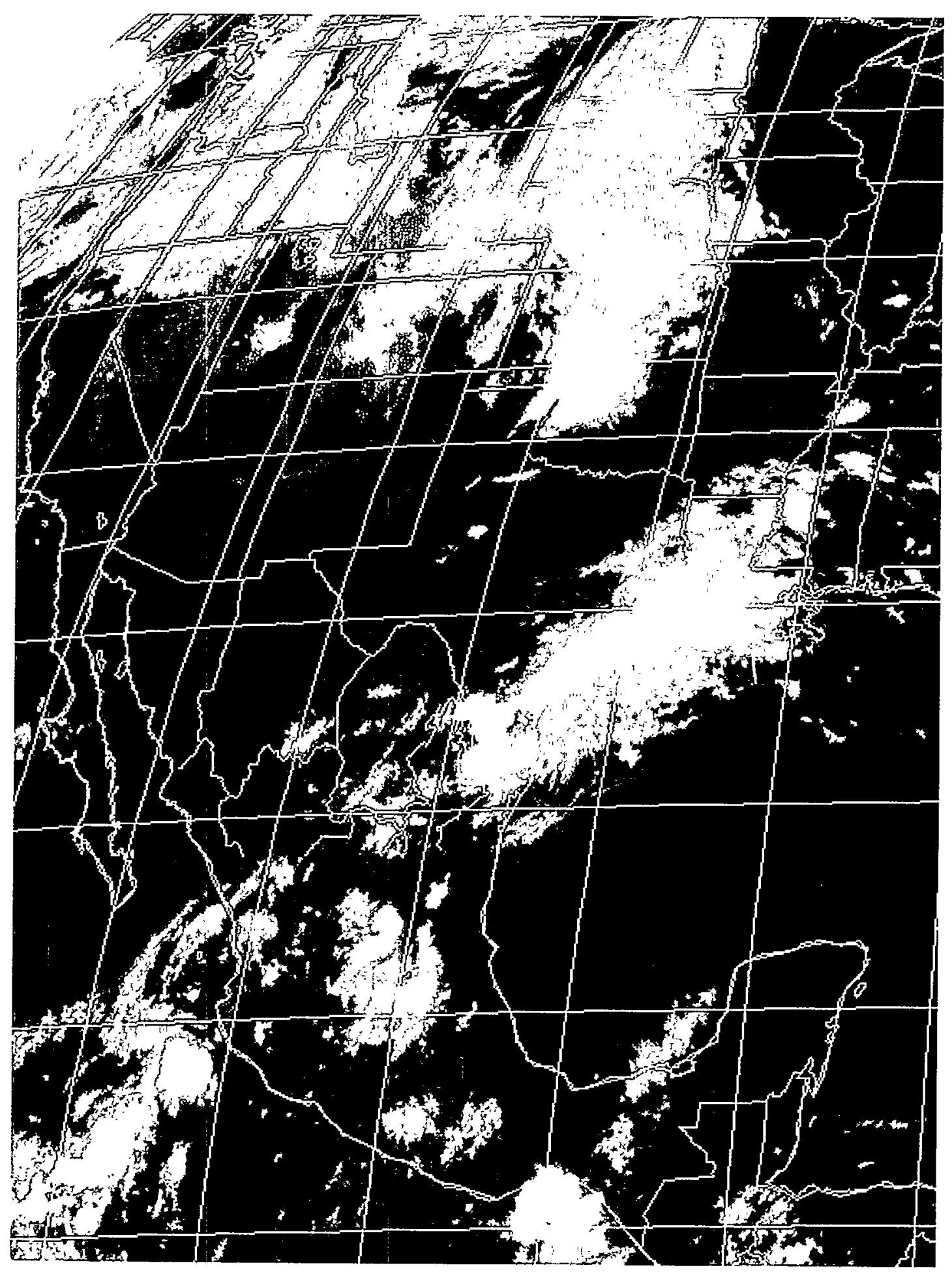

Figure 5.31: Infrared satellite image from GOES-8. The image was acquired near the beginning of the first Blackbeard arm period on 19 September 1996. The image shows that the CID source region occurred in close spatial proximity to cold clouds. 
recordings of TIPPs, that the events emit very powerful radiation up to a frequency of at least $166 \mathrm{MHz}$ (the data presented here were bandlimited by a 28 to $38 \mathrm{MHz}$ bandpass filter). Secondly, the events produce powerful field change and magnetic field signals (the NLDN relies primarily on crossed loop antennas that are sensitive to magnetic field changes). Events presented here were detected from ranges of a few tens of $\mathrm{km}$ to an impressive $2950 \mathrm{~km}$ (the latter detections were made on $11 \mathrm{Sep}$. by the NLDN station in Minot, ND). Finally, the events occurred in relative temporal isolation. The TIPP recordings did not contain any other noteworthy VHF transients in their $7 \mathrm{~ms}$ record lengths. Of the 111 millisecond-duration $\mathrm{HF}$ recordings on 11 Sep., only two records contained other transient signals. None of the four groundbased recordings on 19 Sep. contained other transient signals. The production of powerful $\mathrm{RF}$ radiation and large amplitude field change signals in isolation from other thunderstorm phenomena are the primary defining characteristics of both NPBPs and TIPP events, suggesting that all of the recorded emissions were produced by CIDs.

The third Blackbeard TIPP event of the pass on 11 Sep., which was also recorded by the LANL HF station and multiple NLDN stations, was the first instance of a simultaneous detection of a TIPP event from both the ground and space. The event has provided conclusive evidence of a tie between large amplitude field change pulses and the radio frequency radiation that produces SIPP and TIPP events.

The heights of the sources recorded from tropical cyclone Fausto on Sep. 11 were on the order of $17 \mathrm{~km}$ AGL. The heights of the sources recorded from the remnants of Fausto on Sep. 19 were on the order of $15 \mathrm{~km}$. Because both source regions appear to have been over water or very close to the coast, the corresponding heights above 
sea level were probably also close to 17 and $15 \mathrm{~km}$ respectively. These observed altitudes were significantly higher than $\mathrm{CDD}$ source heights calculated for events that were recorded from the southwestern convective thunderstorms that were observed and described in Chapter 4. The events from those thunderstorms occurred at heights between 8 and $11 \mathrm{~km}$ MSL.

The high source heights measured from tropical cyclone Fausto are consistent with Blackbeard TIPP data, however. The largest pulse separation detected by Blackbeard has been approximately $120 \mu$ s. Quite a few events with separations greater than $100 \mu$ s have been recorded. For a source and its reflection to produce a separation as large as $120 \mu \mathrm{s}$, the source must be at an altitude of at least $18 \mathrm{~km}$ above ground level. As described in Chapter 1, the time separation between the pulses of a TIPP place a lower bound on the source height. If the satellite observes a source and its reflection from directly overhead, the source height above ground level has to be equal to the speed of light times one half of the pulse time separation. If the satellite observes the source from an angle off of nadir, then the source height has to be even greater in order to produce the same pulse time separation. The observations presented in the previous two chapters indicate that different types of storms may host $\mathrm{CID}$ events and that the events may occur at quite different altitudes within those storms. The events produce similar radio emissions, however. 


\section{CHAPTER 6}

\section{CID WAVEFORMS AND PHENOMENOLOGY}

The previous two chapters described observations of distinct, isolated thunderstorm electrical discharges that were recorded from three southwestern thunderstorms and from tropical cyclone Fausto during the summer of 1996. The sources of these discharges were dubbed CIDs. CID discharges are distinct from the intracloud and cloud-to-ground lightning discharges that are most often recorded from thunderstorms. In this chapter, the electric field change waveforms and broadband RF emissions from CDs will be discussed in detail and compared to those from other lightning processes. Also the spatial and temporal phenomenology of the $\mathrm{CDD}$ source region will be discussed in the context of previous thunderstorm electrical observations.

\subsection{CID Electric Field Change EMISSIONS}

CID electric field change waveforms, dubbed NPBPs by Willett et al. [1989], represent a distinct category of thunderstorm electric field change emissions. Characteristics of the emissions clearly distinguish NPBPs from other intracloud 
lightning processes and cloud-to-ground processes. The following observations summarize the distinguishing characteristics of NPBPs. The events were most often recorded as singular, isolated pulses within records that were many milliseconds in duration. All other types of lightning processes were observed to produce tens to hundreds of detectable pulses in records of the same length. NPBPs had large characteristic peak amplitudes that were comparable to those from return strokes and nearly three times greater than those from other intracloud pulses. Also, NPBPs had characteristically short risetimes and durations: typically $2 \mu$ s and $5 \mu$ s respectively. Finally, NPBP electric field change waveforms were always accompanied by very powerful broadband $\mathrm{RF}$ radiation that was on average $20 \mathrm{~dB}$ more powerful than the RF emitted from other types of lightning discharges. Together, these characteristics provide a general description of the field change emissions from CIDs. This section provides a detailed account of NPBP electric field change waveforms recorded from CIDs. NPBP waveform characteristics are summarized in Table 6.1. The corresponding RF emissions are the topic of the following section.

The NPBP electric field change observations described in this section were recorded from $24 \mathrm{CIDs}$ that occurred during three isolated, nighttime, southwestern thunderstorms in the summer of 1996 . The observations were described in detail in Chapter 4. All of the CIDs occurred at altitudes between 8 and $11 \mathrm{~km} \mathrm{MSL}$, and at ranges between 80 and $460 \mathrm{~km}$ from the receivers that recorded them. The CIDs occurred in the general vicinity of cloud-to-ground lightning activity and in close proximity to high reflectivity ( $>40 \mathrm{dBZ}$ ) thunderstorm regions. 


\begin{tabular}{|l|l|l|}
\hline NPBP Waveform Characteristics: & & \\
\hline Risetime (10-90\%) & $2.3 \pm 0.8$ & {$[\mu \mathrm{s}]$} \\
FWHM initial $\Delta \mathrm{E}$ pulse & $4.7 \pm 1.3$ & {$[\mu \mathrm{s}]$} \\
Entire Duration & $25.8 \pm 4.9$ & {$[\mu \mathrm{s}]$} \\
Initial peak $\Delta \mathrm{E}$ & $9.5 \pm 3.6$ & {$[\mathrm{~V} / \mathrm{m} @ 100 \mathrm{~km}]$} \\
Overshoot peak $\triangle \mathrm{E}$ & $-3.9 \pm 1.6$ & {$[\mathrm{~V} / \mathrm{m} @ 100 \mathrm{~km}]$} \\
NPBP Initial Peak Amp. / Overshoot Peak Amp. & 2.7 & \\
Ratio of Peak Amplitudes (NPBP / CG Stroke) & 0.71 & \\
Ratio of Peak Amplitudes (NPBP / IC Stroke) & 2.6 & \\
\hline
\end{tabular}

Table 6.1: Summary of mean measured electric field change characteristics of narrow positive bipolar pulses recorded during this study.

Examples of NPBP waveforms were shown in Figures 4.1, 4.2, 4.3, 4.4, and 4.6. All NPBP electric field waveforms recorded during the 1996 summer thunderstorm campaign were bipolar with an initially-positive slope. No initially-negative, but otherwise NPBP-like pulses were observed. In their study of NPBPs, Willett et al. [1989] described observations of three narrow negative bipolar pulses (NNBPs) that were emitted by thunderstorms and were similar to NPBPs except for their polarity. Medelius et al. [1991] observed 156 negative pulses and only 10 positive pulses, approximately the reciprocal of the ratio observed by Willett et al. [1989]. If NNBPs occurred in the three storms that were observed during this study, they would not have been detected as efficiently as NPBPs. This is because the field change meters were 
configured to trigger only on positive-slope waveforms at a relatively high-amplitude positive thresholds (typically $1.5 \mathrm{~V} / \mathrm{m}$ ). The only way a NNBP could have triggered one of the systems would have been if the positive overshoot following the initial negative pulse had reached the trigger threshold of one of the field change meters. Of the 24 NPBPs recorded, 11 had overshoot amplitudes that were greater than the trigger thresholds at the FC1 station. Thus if it is assumed that NNBPs have an amplitude distribution similar to that for NPBPs, but with the polarity reversed (an assumption consistent with the observations of Willett et al. [1989]), then the FC1 field change station would have had a $46 \%$ detection efficiency for NNBPs relative to NPBPs. The other field change stations would have had similar detection efficiencies. Based on this efficiency it is likely that a number of NNBPs (on the order of 10) would have been observed if they occurred as frequently as NPBPs and if, indeed, they had similar amplitude distributions. Since no NNBPs were observed, it is concluded that negative narrow bipolar pulses were either much more scarce during the times when the thunderstorms were observed or that they had amplitudes that were significantly smaller than those observed for narrow positive bipolar pulses.

\subsubsection{NPBP Temporal Isolation}

Figure 6.1 shows a single NPBP waveform on multiple time scales. The event was recorded from storm 3 by the Socorro field change meter (FC3). The distance to the source was $161 \mathrm{~km}$. The height of the source was $9.4 \mathrm{~km}$ MSL. The top panel of Figure 6.1 shows a $5 \mathrm{~ms}$ electric field change record for the event. The bipolar pulse 

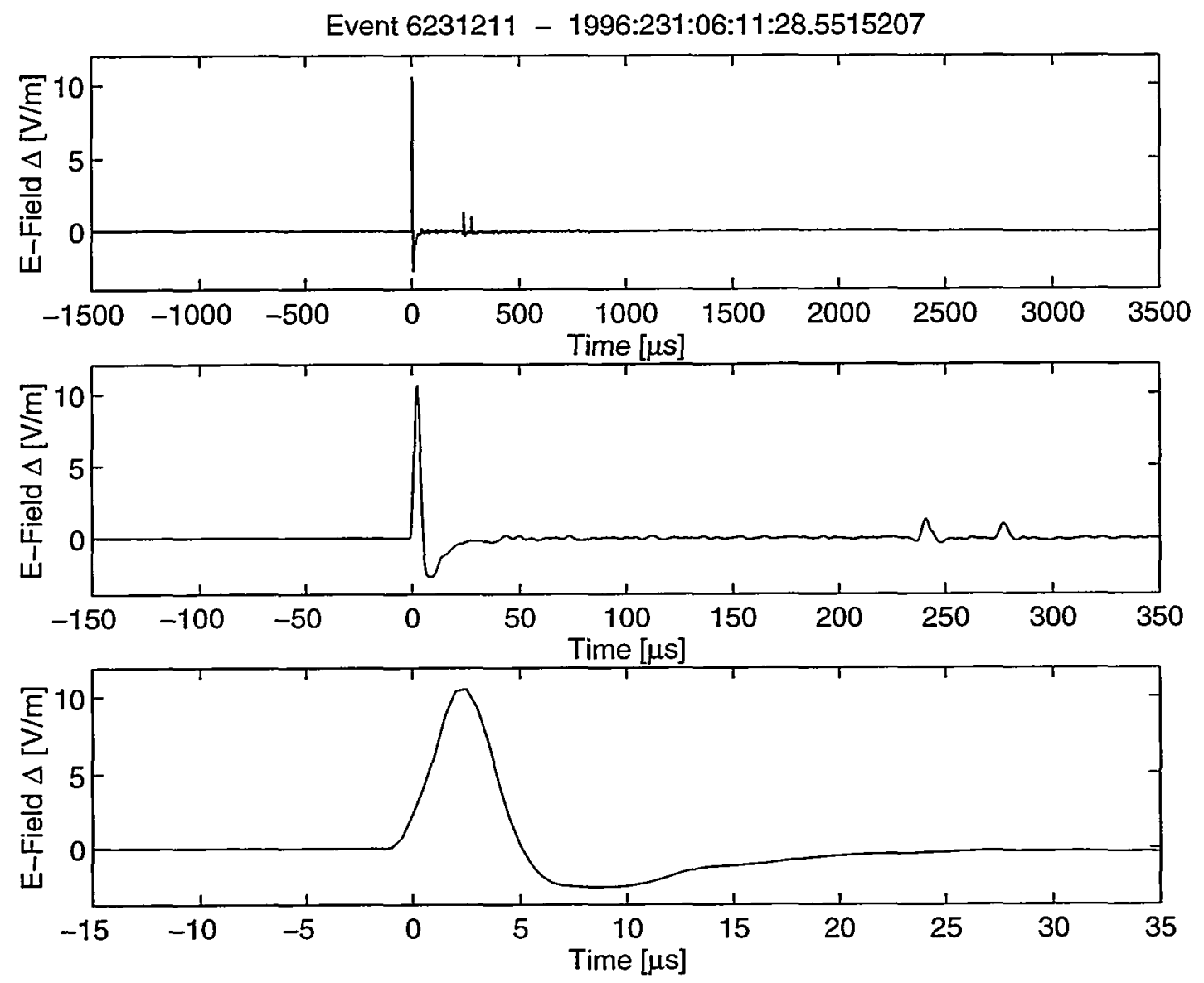

Figure 6.1: Illustrations of a single NPBP on different time scales. The event was recorded by the Socorro electric field change meter (FC3) on the night of 18 August 1996. From top to bottom, the record durations are 5000,500 , and $50 \mu$ s respectively. The NPBP and its reflections from the ionosphere (visible in the upper and middle panels) are the only events within the record. 
is isolated in the record except for its reflections from the ionosphere and earth, which occur at delays of 237 and $273 \mu$ s from the trigger time (time zero in the plot). The center panel shows the top NPBP magnified in time by a factor of 10 . The bottom panel shows the top NPBP magnified in time by a factor of 100. A characteristic of the NPBP in Figure 6.1 that is immediately apparent is its isolation from other pulses (except its own reflections from the earth and ionosphere). The pulse is a solitary occurrence within the $5 \mathrm{~ms}$ field change record. Electric field change records from other types of lightning processes always had multiple field change pulses within records of the same length.

The three field change stations operated during the summer of 1996 most often acquired records that were either $4.1,5.0$ or $8.2 \mathrm{~ms}$ in duration. During storm 2 the LANL FC1 field change meter acquired longer, $50 \mathrm{~ms}$ records. When NPBPs were recorded, they and their reflections from the ionosphere were usually the only features within the records. In 17 of the 24 NPBP electric field change records acquired by the field change meters, the NPBP was the only detectable event within a $4.1 \mathrm{~ms}$ window around the trigger time. In the other 7 records, the extra pulses were from intracloud activity that occurred after the NPBP. An example of such a record is shown in Figure 6.2, which shows data from the Socorro (FC3) field change meter. The NPBP at the trigger point occurred at an altitude of $8.9 \mathrm{~km} \mathrm{MSL}$ and at a range of $152 \mathrm{~km}$ from the recording station. The smaller, positive pulses that occurred after the NPBP are typical of the field change pulses recorded from "normal" intracloud lightning activity. Such IC pulses were also shown in Figure 4.7 and have been characterized, for example, by Kitagawa and Brook [1960], Krider et al. [1979], and Shao and 


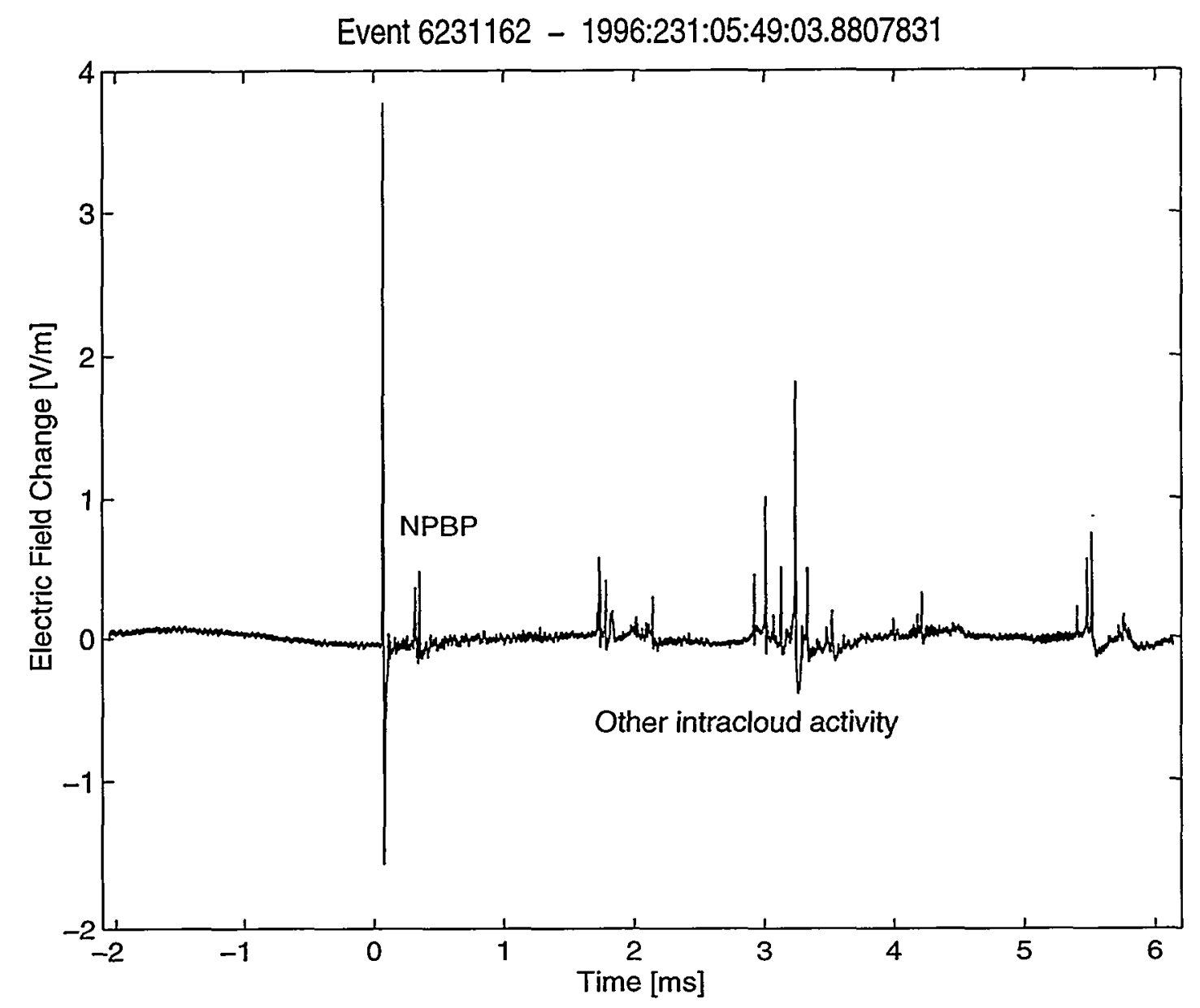

Figure 6.2: $\quad$ Electric field change waveform of an NPBP and intracloud flash that occurred within a few milliseconds of each other. NPBPs occurred within 4 to $8 \mathrm{~ms}$ of other lightning activity in approximately $25 \%$ of CID electric field change recordings. In all of these cases, the other activity was intracloud activity that occurred after the NPBP. 
Krehbiel [1996]. The pulses are initially-positive with inter-pulse separations that range from microseconds to milliseconds during the duration of the flash, which typically lasts several hundred milliseconds. In all 24 NPBP electric field change records, the NPBP was the only event within a $1 \mathrm{~ms}$ window on either side of the trigger time (again, ignoring its own ionospheric reflections).

On time scales greater than the record lengths in this study, NPBPs were usually temporally isolated from other detectable transients from thunderstorms on time scales comparable to the times between normal lightning discharges in the storms, namely from several seconds to several tens of seconds. This is a qualitative observation and is based largely on data recorded by the NMIMT (FC2) field change meter. FC2 had a very short re-arm time of a few milliseconds. The short re-arm time allowed the station to record data almost continuously during bursts of electrical activity.

In nearly $75 \%$ of the NPBP waveforms recorded, the NPBP was the only detectable transient. This result shows that occurrence of the events in isolation can be a powerful means of distinguishing the events from other intracloud pulses. Obviously the events can occur in conjunction with other lightning activity, though. It was not possible to determine from the data whether the 7 of 24 records that contained both NPBPs and other IC activity were likely to have resulted from random chance or from related activity. The fact that the NPBPs occurred before the IC pulses in all 7 cases suggest that there may, in fact, be a causal relationship. In a private communication, Marx Brook noted that he has observed NPBPs immediately prior to intracloud discharges on a number of occasions. The data seem to suggest 
that NPBPs may initiate normal intracloud discharges some fraction of the time, but that more often than not, they occur in isolation.

\subsubsection{NPBP Amplitude}

The mean range-normalized initial peak amplitude for the 24 NPBP waveforms was $9.5 \pm 3.6 \mathrm{~V} / \mathrm{m}$ at $100 \mathrm{~km}$. This value is consistent with the value of $8.0 \pm 5.3$ $\mathrm{V} / \mathrm{m}$ at $100 \mathrm{~km}$ for $18 \mathrm{NPBPs}$ observed by Willett et al. [1989] from a distance of 45 $\mathrm{km}$. In both this study and the study by Willett et al. [1989], amplitudes were normalized for range by assuming a $1 / \mathrm{r}$ distance attenuation relationship for recorded waveforms. This is the relationship that describes attenuation of the radiation field term in the general field equations [Uman, 1987, b]. It was assumed that recorded NPBP electric field waveforms were from source radiation fields because the scale size of CD discharges (calculated to be less than $1 \mathrm{~km}$, as will be shown in Chapter 7) was much smaller than the ranges to the events from the stations that observed them $(80$ to $460 \mathrm{~km}$ ). Additionally it has been shown that electric field changes from return strokes, which have much longer durations than NPBPs and spatial dimensions greater than $5 \mathrm{~km}$, consist almost entirely of a radiation field component at distances greater than $50 \mathrm{~km}$ [Uman et al., 1975; Lin et al., 1979].

The mean range-normalized undershoot peak amplitude for the 24 NPBPs was $3.9 \pm 1.6 \mathrm{~V} / \mathrm{m}$ at $100 \mathrm{~km}$. The mean ratio of the initial positive peak amplitude to that of the negative undershoot peak amplitude was 2.7. Willett et al. [1989] reported a significantly smaller mean peak undershoot amplitude of $-1.2 \pm 1.3 \mathrm{~V} / \mathrm{m}$ at $100 \mathrm{~km}$ 
and a correspondingly larger mean amplitude ratio of 8.9. NPBP waveforms published by Le Vine [1980] exhibited amplitude ratios similar to those observed by Willett et al. [1989]. Ratios observed by Medelius et al. [1991] were smaller, with a mean value of 4.6. These results are summarized in Table 6.2. The discrepancies between the amplitude observations presented here and those presented by previous researchers are believed to be attributable to propagation effects between the sources and receivers.

\begin{tabular}{|l|l|l|l|}
\hline & Initial Peak $\Delta \mathrm{E}[\mathrm{V} / \mathrm{m}]$ & Undershoot Peak $\Delta \mathrm{E}[\mathrm{V} / \mathrm{m}]$ & Ratio \\
\hline Present Study & $9.5 \pm 3.6$ & $-3.9 \pm 1.6$ & 2.7 \\
\hline Le Vine [1980] & - & - & $8^{*}$ \\
\hline Willett et al. [1989] & $8.0 \pm 5.3$ & $-1.2 \pm 1.3$ & 8.9 \\
\hline Medelius et al. [1991] & - & - & 4.6 \\
\hline
\end{tabular}

"Estimated by this author based on figures.

Table 6.2: Comparison of initial and overshoot NPBP amplitudes.

A model was adapted from Volland [1995] to study the predicted effect of the propagation of NPBPs over a finitely-conducting earth. Observations described in this paper were made from distances of 80 to $460 \mathrm{~km}$ over dry soil, which has a relatively poor conductivity of $1 \times 10^{-4}$ to $5 \times 10^{-4} \mathrm{~S} / \mathrm{m}$ at LF [Smith, 1991]. Willett et al. [1989] and Medelius et al. [1991] made their observations of NPBPs from distances on the order of $50 \mathrm{~km}$ over salt water, which has a much higher conductivity 
of $4 \mathrm{~S} / \mathrm{m}$ [Volland, 1995]. The propagation model had the following complex transfer function $\left(T_{o}\right)$ :

$$
T_{o}(\omega, r)=\frac{1}{(1-j y / \mathrm{A})(1-2 j \mathrm{~A} y)}
$$

where $\omega$ is the angular frequency of the electromagnetic wave, $r$ is the propagation distance, $\mathrm{A}$ is a constant equal to $0.49+\mathrm{j} 0.85$, and $y$ is given by the following relation:

$$
y=\frac{\omega}{\sqrt{2 c \sigma_{e} / \varepsilon_{o} r}}
$$

In equation $6.2, \sigma_{e}$ is the conductivity of the ground.

The magnitude of the complex transfer function $\left(T_{o}\right)$ as a function of frequency for $\sigma_{e}=4 \times 10^{-4} \mathrm{~S} / \mathrm{m}$ and $r=100 \mathrm{~km}$ is shown in Figure 6.3. The maximum frequency of $500 \mathrm{kHz}$ corresponds to the upper cutoff of the electric field change meter response for the LANL FC1 station. Figure 6.4 shows the effect of applying the transfer function to a narrow positive bipolar pulse. The upper panel of the figure shows a synthesized pulse with mean characteristics that match those of NPBPs recorded by Willett et al. [1989]. The lower panel shows the pulse after being filtered. The pulse retains approximately the same normalized amplitude, but the amplitude ratio is reduced. The pulse also becomes wider and does not rise as sharply. These 


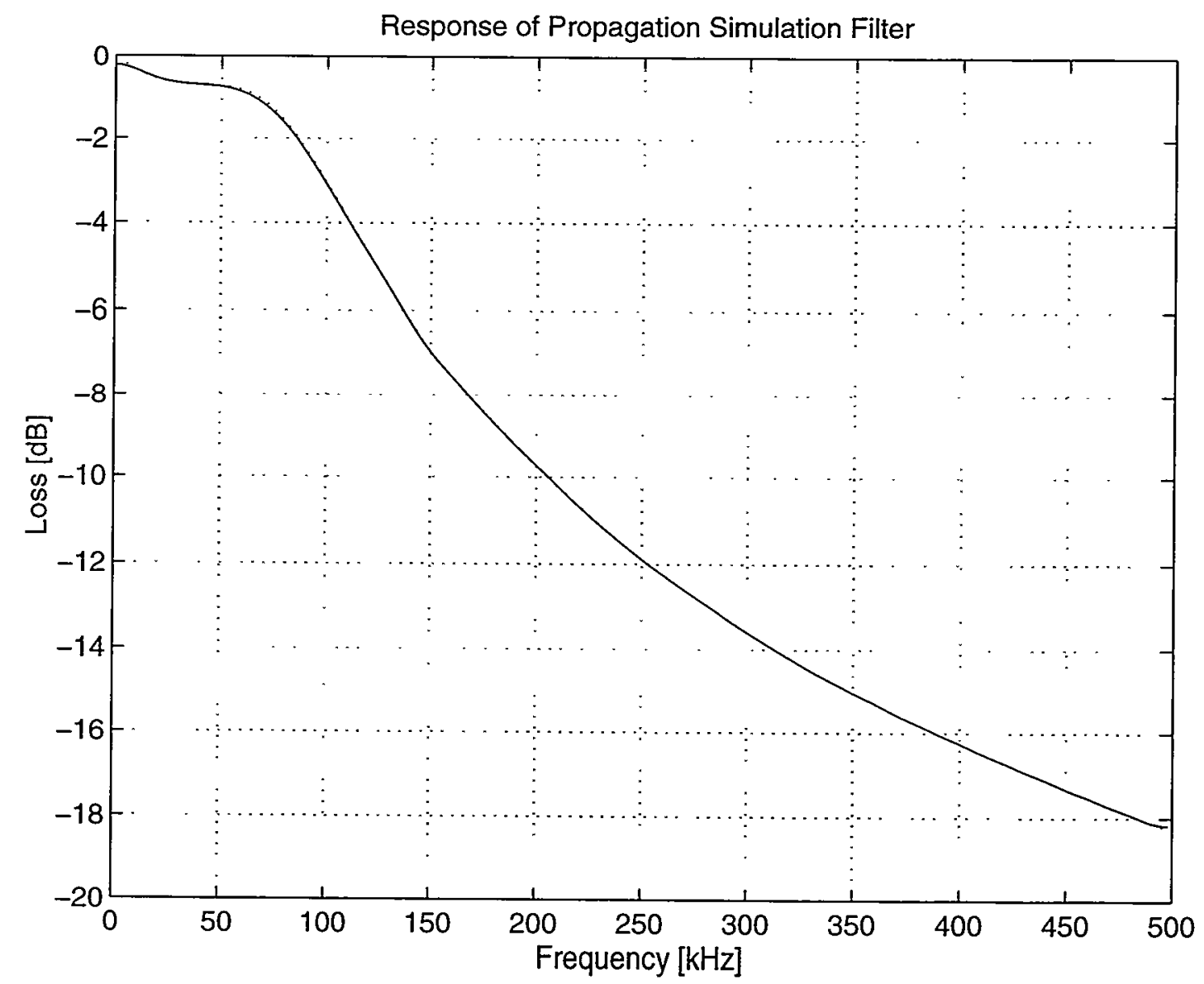

Figure 6.3: Frequency response of the filter used to model the propagation of electric field change signals over the finitely-conducting earth. A propagation distance of $100 \mathrm{~km}$ and a conductivity of $4 \times 10^{-4} \mathrm{~S} / \mathrm{m}$ were assumed. 

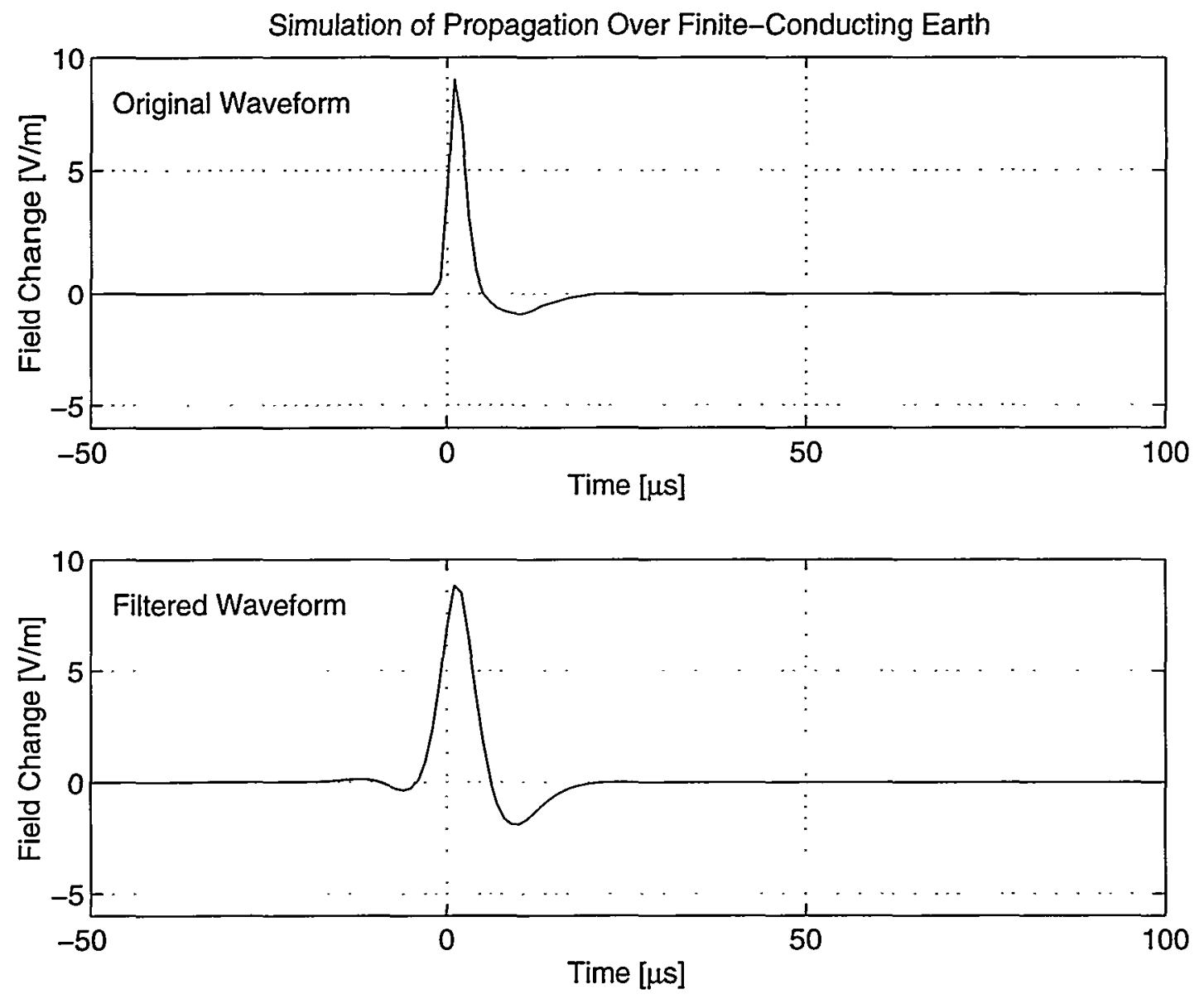

Figure 6.4: The NPBP in the upper panel, which has characteristics matching pulses recorded by Willett et al. [1989], was passed through a filter to simulate propagation over a finite-conductivity earth. The resulting waveform is shown in the lower panel. The pulse was broadened and the amplitude ratio between the positive and negative portions was reduced. 
characteristics are discussed in the following section. Table 6.3 provides a summary of the pulse characteristics before and after filtering and also summarizes the characteristics of NPBPs observed during the present study. The table shows that propagation over the finitely-conducting earth is capable of producing the differences between the NPBPs observed by Willett et al. [1989] and those observed during the study described here.

\begin{tabular}{|l|l|l|l|}
\hline & $\begin{array}{l}\text { NPBP from } \\
\text { Willett et al. [1989] }\end{array}$ & $\begin{array}{l}\text { NPBP after } \\
100 \mathrm{~km} \text { propagation }\end{array}$ & NPBPs from \\
present study
\end{tabular}

Table 6.3: Results from propagation model. The differences between previous observations and the observations described here are shown to be consistent with long-distance propagation over finitely-conducting earth.

The peak electric field change amplitudes of NPBPs were comparable to those of return stroke waveforms. Return strokes produce the largest electric field changes in thunderstorms. A comparison of 8 NPBPs and 11 negative CG return stroke 
waveforms recorded by FC3 from storm 3 showed that NPBPs peak amplitudes, on average, were 0.71 times as large as peak negative CG return stroke amplitudes. The field change meter was located approximately $170 \mathrm{~km}$ from the storm that produced the events. The mean normalized peak amplitude values for NPBPs and negative CGs were $6.4 \pm 3.3 \mathrm{~V} / \mathrm{m}$ and $9.0 \pm 4.5 \mathrm{~V} / \mathrm{m}$ at $100 \mathrm{~km}$ respectively. The observed ratio of 0.71 compares favorably to the ratio of 0.72 computed by Willett et al. [1989] in a similar study. Le Vine [1980] also made this comparison, but observed a significantly lower ratio of 0.3 . These results are summarized in Table 6.4.

\begin{tabular}{|l|l|l|l|l|l|}
\hline & $\begin{array}{l}\text { Peak NPBP } \\
\Delta \mathrm{E}[\mathrm{V} / \mathrm{m}]\end{array}$ & $\begin{array}{l}\text { Peak CG } \\
\Delta \mathrm{E}[\mathrm{V} / \mathrm{m}]\end{array}$ & $\begin{array}{l}\text { NPBP/CG } \\
\Delta \mathrm{E} \text { Ratio }\end{array}$ & $\begin{array}{l}\text { Peak IC } \\
\Delta \mathrm{E}[\mathrm{V} / \mathrm{m}]\end{array}$ & $\begin{array}{l}\text { NPBP/C } \\
\Delta \mathrm{E} \text { Ratio }\end{array}$ \\
\hline Present Study (storm 3 only) & $6.4 \pm 3.3$ & $9.0 \pm 4.5$ & 0.71 & $2.5 \pm 0.8$ & 2.6 \\
\hline Le Vine [1980] & - & - & 0.33 & - & - \\
\hline Willett et al. [1989] & $8.0 \pm 5.3$ & $11.1 \pm 3.9$ & 0.72 & - & - \\
\hline Cooray and Lundquist [1982] & - & $11.2 \pm 5.6$ & - & - & - \\
\hline Krider and Guo [1983] & - & $8.8 \pm 4.0$ & - & - & - \\
\hline Master et al. [1984] & - & $6.2 \pm 3.4$ & - & - & - \\
\hline
\end{tabular}

Table 6.4: Comparisons of the peak electric field change amplitudes of NPBPs, cloud-to-ground waveforms, and intracloud waveforms.

The mean observed peak return stroke electric field change amplitude of $9.0 \pm$ $4.5 \mathrm{~V} / \mathrm{m}$ at $100 \mathrm{~km}$ for the $11 \mathrm{CGs}$ is comparable to previous observations of lightning 
by Cooray and Lundquist [1982], Krider and Guo [1983], and Master et al. [1984].

See Table 6.3 for a summary of their results and a comparison to the results presented here. The similarities between the results show that the absolute electric field change amplitudes presented here are consistent with those of previous researchers.

The peak amplitudes of pulses from intracloud lightning strokes recorded by FC3 during storm 3 were also studied. The mean normalized peak field change value for 12 intracloud pulses that triggered the system was $2.5 \pm 0.8 \mathrm{~V} / \mathrm{m}$ at $100 \mathrm{~km}$. The ratio of the mean NPBP peak amplitude to that of the mean peak IC pulse amplitude was 2.6. This ratio is probably quite sensitive to triggering threshold since the typical thresholds used at the stations were set to capture only the brighter intracloud pulses. A lower threshold would have certainly increased the ratio. The IC results are summarized in Table 6.3.

In the 7 (out of 24) electric field change records that featured NPBP pulses with an accompanying coda of intracloud pulses, pulse amplitudes were used, in part, to distinguish the NPBPs as field change emissions from CIDs and not emissions from other intracloud activity. The waveform in Figure 6.2 was an example of this. In the figure, the amplitude of the NPBP is significantly greater than that of other intracloud pulses. Figures $4.1,4.2$, and 4.8 are also useful for illustrating the relative amplitudes between NPBPs and other IC pulses, since the field change waveforms in the three figures were recorded by the same receiver (FC1) during the same storm (storm 3). Figures 4.1 and 4.2 showed NPBP field change pulses. Figure 4.8 showed IC field change pulses. Note that in these three figures and in Figure 6.2, the electric field 
change values were not range-normalized. The values are as recorded by their respective receiving systems.

\subsubsection{NPBP Duration and Risetime}

The mean FWHM of 24 NPBP initial pulses was $4.7 \pm 1.3 \mu$ s. This value is significantly larger than the values reported by Willett et al. [1989] and Medelius et al. [1991]. Willett et al. [1989] reported mean FWHM values of $2.4 \pm 1.4$ and $1.9 \pm 1.0$ $\mu$ for two storms observed from the Kennedy Space Center (KSC), one of which was centered at a distance of $45 \mathrm{~km}$ from the point of observation. The distance to the other storm was unknown. Medelius et al. [1991] reported FWHM values of 1.83 and $1.60 \mu \mathrm{s}$ for narrow bipolar pulses of initially-negative and initially-positive polarities respectively. The distances to the sources from their point of observation at KSC were not known, but were believed to fall within the range from 1.5 to $20 \mathrm{~km}$. These results are summarized in Table 6.5. Like the amplitude discrepancies described in the previous section, the longer-duration FWHM values that were observed during the present study can be attributed to propagation effects. The LF/VLF propagation model presented in Section 6.1.2 showed that NPBP pulses effectively elongate as a result of long-distance propagation over a finitely conducting earth. The sample pulse increased in FWHM from $2.5 \mu$ s to $4.7 \mu$ s.

The mean overall NPBP waveform duration was $25.8 \pm 4.9 \mu$ s. Duration is

defined here as the time from when the initial portion of the waveform crosses $10 \%$ of 
the peak positive amplitude to the time when the negative undershoot of the waveform crosses $10 \%$ of the negative peak amplitude during its return to zero. The mean measured duration is in agreement with the observations of Willett et al. [1989], who reported NPBP durations of 20 to $30 \mu$ s. Pulse duration was not affected significantly in the ionospheric propagation model. The duration of the sample pulse increased from $23 \mu$ s to $27 \mu \mathrm{s}$.

\begin{tabular}{|l|l|l|l|}
\hline & FWHM $[\mu \mathrm{s}]$ & Duration $[\mu \mathrm{s}]$ & Risetime $[\mu \mathrm{s}]$ \\
\hline Present Study & $4.7 \pm 1.3$ & $25.8 \pm 4.9$ & $2.3 \pm 0.8$ \\
Willett et al. [1989] & - & $10-20$ & - \\
& $2.4 \pm 1.4($ storm 1) & $20-30$ & - \\
Medelius et al. [1991] & $1.9 \pm 1.0$ (storm 2) & & \\
& $1.63 \pm 0.63(-$ pol.) & - & $1.54 \pm 1.04(-$ pol.) \\
& & & $1.82 \pm 0.87$ (+ pol.) \\
\hline
\end{tabular}

Table 6.5: Comparison of NPBP FWHMs, Durations, and Risetimes.

The mean NPBP risetime (10 - 90\%) was $2.3 \pm 0.8 \mu \mathrm{s}$. This result compares to risetimes of $1.54 \pm 1.04$ and $1.82 \pm 0.87$ computed for initially-negative and initiallypositive pulses recorded by Medelius et al. [1991]. Willett et al. [1989] did not report pulse risetimes, but based on their published waveforms, the risetimes appear to be consistent with those from Medelius et al. [1991]. The discrepancy between the 
previous measurements and those presented here can again be ascribed to propagation effects, as shown in Table 6.3.

\subsection{CID HF AND VHF RADIO EMISSIONS}

Like the electric field change signals described in the previous section, the $\mathrm{RF}$ emissions from CIDs were distinct in comparison to other thunderstorm electrical emissions. Broadband $\mathrm{HF}$ data ( 3 to $30 \mathrm{MHz}$ ) were acquired in conjunction with all 24 of the NPBP electric field change signals that were described in Section 6.1. Examples of four of the HF waveforms with their associated spectrograms were shown in Figures 4.1, 4.2, 4.4, and 4.6. All of the HF waveforms were recorded by the primary HF data acquisition system (HF1). Broadband RF emissions of CIDs have also been recorded by the Blackbeard VHF satellite receiver since 1993 in the form of TIPP events. Spectrograms of TIPPs recorded from tropical cyclone Fausto were shown in Figures 5.1, 5.4, 5.5, 5.18, and 5.19. Although these figures only show data in the range from 28 to $38 \mathrm{MHz}$, Blackbeard has shown than CIDs produce bright $\mathrm{RF}$ radiation up to its instrumental limit of $166 \mathrm{MHz}$.

Some of the distinct characteristics of $\mathrm{CD}$ RF emissions include the following: The emissions occurred as singular, isolated RF bursts within 1 millisecond data records. The events had short durations of a few microseconds. On average the events were much more powerful than the RF emissions from other intracloud

processes and cloud-to-ground processes. Also the CD RF emissions did not exhibit 
the one over frequency squared spectral roll off that usually characterizes thunderstorm lightning emissions. Observations of $\mathrm{CD}$ RF emissions are summarized in Table 6.6. The Blackbeard VHF observations are from Holden et al. [1995] and Massey and Holden [1995].

\begin{tabular}{|l|l|l|}
\hline CID RF Characteristics: & & \\
\hline Duration (Ground-Based HF) & $2.8 \pm 0.8$ & {$[\mu \mathrm{s}]$} \\
Duration (Blackbeard VHF) & 5.0 & {$[\mu \mathrm{s}]$} \\
Peak E (HF) & $2.4 \pm 1.1$ & {$[\mathrm{mV} / \mathrm{m} @ 10 \mathrm{~km}$ in 1 kHz BW] } \\
Ratio of Peak Amplitudes (HF) & 9.9 & \\
(NPBP / CG Stroke) & & \\
Ratio of Peak Amplitudes (HF) & 29 & \\
(NPBP / IC Stroke) & & \\
\hline
\end{tabular}

Table 6.6: Summary of mean measured RF characteristics of CIDs.

\subsubsection{CID RF Temporal Isolation}

Like the electric field change emissions from CIDs that were described in Section 6.1, the RF emissions from CIDs occurred as isolated events. Of the $24 \mathrm{CID}$ events recorded by the LANL HF stations, none contained other detectable transient thunderstorm emissions within their $1 \mathrm{~ms}$ time records, except when their own reflections from the earth and ionosphere were recorded. 
HF records from other types of lightning processes had multiple pulses within records of the same length. In 7 recordings of negative CGs from storm 3, the average number of detectable HF pulses in the one millisecond record length was 32 . The minimum and maximum numbers of pulses were 16 and 70 . In 15 recordings of intracloud discharges from storm 3, the average number of detectable HF pulses in the one millisecond record length was 17 . The minimum and maximum numbers were 4 and 60. These results are summarized in Table 6.7.

\begin{tabular}{|l|l|l|l|}
\hline & Minimum \# Pulses & Mean \# Pulses & Maximum \# Pulses \\
\hline CIDs (24) & 1 & 1 & 1 \\
\hline Cloud-to-ground flashes (7) & 16 & 32 & 70 \\
\hline Intracloud flashes (15) & 4 & 17 & 60 \\
\hline
\end{tabular}

Table 6.7: Numbers of detectable RF pulses in 1 millisecond records. CIDs are shown to be temporally isolated compared to other lightning types.

Counts of pulses for this study were determined in the following manner. First, HF waveforms were pre-whitened as described in Section 5.1. Then, a rolling determination of mean power within a $1 \mu \mathrm{s}$ window was performed. Each time the smoothed power crossed a threshold $3 \mathrm{~dB}$ above the mean power in the waveform, a pulse was counted. The $1 \mu$ s window size corresponds to the approximate duration of a typical broadband RF lightning burst. Without smoothing, each burst would cross the threshold many times. The observed mean pulse numbers for intracloud and 
cloud-to-ground flashes correspond to pulse rates of $1.7 \times 10^{4}$ and $3.2 \times 10^{4}$ pulses per second respectively. Allowing for the fact that pulse counting is greatly influenced by receiver characteristics and threshold settings, these rates are in agreement with an average flash rate of $1 \times 10^{4}$ pulses per second reported by Oetzel and Pierce [1969, a] and Pierce [1977] for observations at frequencies between 30 and $100 \mathrm{MHz}$.

In all $24 \mathrm{CDD} R$ F records, the singular pulses were isolated within time spans that were $1 \mathrm{~ms}$ in duration. Had longer records been acquired, it would not have been surprising to find that observations of RF pulses mirrored the observations of electric field change signals that were described in Section 6.1. Recall that 7 out of 24 NPBPs were followed by intracloud field change pulses that occurred a few milliseconds later. Intracloud electric field change pulses are often associated with RF pulses, as shown in Figure 4.8. This observation is consistent with the comment by Krider et al. [1979] that "the largest RF emissions usually occur in conjunction with pulses in the electric field, but the amplitude of the RF in relation to the field is highly variable." The observed isolation of CID RF pulses is consistent with the observation of Willett et al. (1989) that NPBPs normally corresponded to the only detectable HF peaks in their recordings.

Blackbeard data have also indicated that CIDs usually occur in temporal isolation from other thunderstorm electrical emissions. TIPP events were most often the only transient events in records that ranged from $7 \mathrm{~ms}$ to longer than $100 \mathrm{~ms}$ in duration. 


\subsubsection{CID RF Duration}

Durations of $\mathrm{HF}$ emissions from CIDs were computed using a power-weighted centroid width determination. TIPP pulse widths reported by Massey and Holden [1995] were computed in a similar manner. Centroid widths were computed for individual pulses such that they contained half of the energy within the entire pulse. Widths were computed for 20 events (including reflections from the ionosphere) that were recorded by the ground-based HF receivers during storms 1,2 , and 3 . The mean centroid width was $2.8 \pm 0.8 \mu \mathrm{s}$. The median width was $3.2 \mu \mathrm{s}$.

These durations are comparable to, although slightly shorter than, the measured durations of TIPP pulses determined by Massey and Holden [1995]. In their study, the mean pulse centroid width for 183 TIPP low band (28 to $95 \mathrm{MHz}$ ) pulses was 5.0 $\mu \mathrm{s}$. The median duration was $3.8 \mu \mathrm{s}$. As was done for the study of CID durations here, Massey and Holden [1995] included both direct and reflected pulses in their characterization of event durations. The slight bias toward longer RF durations in the Blackbeard data might possibly be related to the fact that Massey and Holden [1995] observed generally different types of thunderstorms than those described in this study. For their study, Blackbeard was armed only over equatorial regions of the earth. The data from tropical cyclone Fausto presented here suggested that CID phenomenology

could be a function of storm type since source heights were significantly higher in that storm (15-17 km MSL) than heights computed for the isolated airmass thunderstorms described in Chapter 4 (8-11 km MSL). 


\subsubsection{CID RF Amplitudes/Power}

The RF emissions from CIDs were very powerful, lasted for a few microseconds, and resembled broadband bursts of noise in that the durations of the events were much longer than the inverse of their bandwidths. HF and VHF measurements of the events from the LANL ground array and Blackbeard have shown that the events produce powerful RF throughout the range of frequencies from 3 to $166 \mathrm{MHz}$. In 1994, Xuan-Min Shao (private communication) made RF and field change observations of a few CID pulses emitted from a close thunderstorm using a broadband system that sampled at $1 \mathrm{Gsample/s}$. His data showed that the spectra of the events appeared bright up to at least $500 \mathrm{MHz}$. A bandwidth of $500+\mathrm{MHz}$ suggests that the sources responsible for the emissions have risetimes that are shorter than $2 \mathrm{~ns}$ (the inverse of the bandwidth). Thus the few microsecond durations of CID RF bursts probably result from a great number of very brief emissions. The process produces emissions that closely resemble broadband noise.

The spectrum for CID events that is suggested by the HF, VHF, and UHF data presented here is markedly different from that which has been reported for lightning in the literature. Summarizing the results of others, Pierce [1977] showed that the radiated power from lightning falls off with frequency in proportion to frequency squared up to $10 \mathrm{MHz}$. Above $10 \mathrm{MHz}$, the radiation from lightning falls off at an even faster rate. CID RF emissions do not fall off with frequency in the manner described by Pierce [1977]. The exact ratio of the power produced by the emissions at HF frequencies to that produced at VHF or UHF frequencies has not yet been 
determined, but qualitatively the emissions appear as bright or at least nearly as bright at very high frequencies.

Broadband HF peak electric fields associated with CDs were larger in amplitude than those produced by cloud-to-ground and intracloud lightning strokes by factors of 10 and 29 respectively. The mean peak electric field from six HF bursts from CIDs recorded during storm 3 was $2.9 \pm 1.1 \mathrm{mV} / \mathrm{m}$, normalized to a range of $10 \mathrm{~km}$ and a 1 $\mathrm{kHz}$ bandwidth centered at $20 \mathrm{MHz}$. The mean normalized peak field associated with 11 negative cloud-to-ground strokes recorded during the same storm was $0.29 \pm 0.11$ $\mathrm{mV} / \mathrm{m}$. The mean peak field associated with three intracloud strokes was $0.10 \pm 0.04$ $\mathrm{mV} / \mathrm{m}$. These results are summarized in Table 6.8. Figures 4.1 and 4.2 (CIDs) and 4.7 and 4.8 (CG and IC strokes respectively) provided illustrations of the large power radiated from CIDs at HF frequencies compared to that radiated from typical lightning processes. All of the events in these figures occurred at approximately the same distance from the receiver. The mean normalized peak electric field for HF bursts associated with CIDs recorded from 11 events recorded during all three storms was $2.3 \pm 1.1 \mathrm{mV} / \mathrm{m}$ at $10 \mathrm{~km}$ in $1 \mathrm{kHz}$ (only line-of-sight pulses were used to characterize amplitudes, not reflected pulses).

Although 10 times larger than peak fields radiated from lightning at $\mathrm{HF}$ frequencies, peak fields from CDs were comparable to those of TIPP events recorded by Blackbeard. Holden et al. [1995] stated that "peak fields from TIPP events are on the order of ten times the comparably measured fields from ground-based measurements of lightning," a result that is consistent with the measurements 
presented here of radio frequency radiation associated with CIDs. Massey and Holden [1995] described a similar result and indicated that peak field values for TIPP events were on the order of a few $\mathrm{mV} / \mathrm{m}$ at $10 \mathrm{~km}$ in a $1 \mathrm{kHz}$ bandwidth, a result also consistent with the observations presented here. More accurate peak field calculations for TIPP events recorded by Blackbeard were not possible because actual source locations and ranges were not known.

\begin{tabular}{|l|l|l|l|l|l|}
\hline & $\begin{array}{l}\text { Peak CID } \\
\text { E }[\mathrm{mV} / \mathrm{m}]\end{array}$ & $\begin{array}{l}\text { Peak CG } \\
\text { E [mV/m] }\end{array}$ & $\begin{array}{l}\text { CID/CG } \\
\text { E Ratio }\end{array}$ & $\begin{array}{l}\text { Peak IC } \\
\text { E }[\mathrm{mV} / \mathrm{m}]\end{array}$ & $\begin{array}{l}\text { CID/IC } \\
\text { E Ratio }\end{array}$ \\
\hline Present Study (storm 3 only) & $2.9 \pm 1.1$ & $0.29 \pm 0.11$ & 10 & $0.10 \pm 0.04$ & 29 \\
\hline
\end{tabular}

Table 6.8: Comparisons of the peak HF electric field amplitudes produced by CIDs, cloud-to-ground lightning strokes, and intracloud lightning strokes. Fields are normalized to a range of $10 \mathrm{~km}$ and a bandwidth of $1 \mathrm{kHz}$.

\subsection{SOURCE REGION PHENOMENOLOGY}

In Chapter 7, the characteristics of CD electric field change waveforms and RF emissions will be used to determine information about the source and source region. Also of interest to the investigation are measurements of where the events occurred and what further information is available about the source region based on measurements presented here and based on the results of other researchers. 
As reported in Chapter 4, the calculated heights of CIDs that occurred in three southwestern thunderstorms ranged from 8 to $11 \mathrm{~km} \mathrm{MSL.} \mathrm{It} \mathrm{was} \mathrm{observed} \mathrm{by}$ Simpson and Scrase [1937], based on in situ measurements, that thunderstorms generally consist of an upper positive region above a lower negative charge region, and that some fraction of the time (roughly one third), a second positive charge region may exist below the main negative charge region. This model for thunderstorm charge distribution has come to be known as the positive dipole or Simpson model. Remote electric field measurements by Malan [1963] indicated that when the three charge regions were represented as point charges, the heights of the regions were 10 , 5 , and $2 \mathrm{~km}$ and the charge magnitudes were $+40,-40$, and $+10 \mathrm{C}$ respectively. Malan [1963] noted that this was a simplification primarily designed to permit quick calculations of electric fields. The observations suggest that CIDs may occur between the upper positive and lower negative thunderstorm charge regions.

Observations of thunderstorms in New Mexico by Workman et al. [1942], Reynolds and Neill [1955], Brook et al. [1962], and Krehbiel et al. [1974], have shown that the main negative charge region in New Mexico thunderstorm is typically centered between 5 and $8 \mathrm{~km}$ MSL. The air temperature between these altitudes is typically $-12^{\circ}$ to $-36^{\circ} \mathrm{C}$ [Workman et al., 1942; Reynolds and Neill, 1955; Jacobson and Krider, 1976]. These observations provide more specific evidence that the CIDs observed occurred between the lower negative and upper positive charge regions. Note that more recent in situ measurements of thunderstorm electric fields, particularly in large mesoscale convective complexes, have indicated that multiple charge layers or regions may exist, and that the positive dipole model is often not an 
accurate representation of thunderstorm charge distribution [Marshall and Rust, 1991; Marshall et al., 1995, a]. A study by Marshall et al. [1995, b] of smaller thunderstorms in New Mexico (probably more like the ones described in this study) showed that multiple charge regions existed in the storms, but that the storms usually had negative charge below about $8 \mathrm{~km}$ with positive charge overhead. It was also observed by Marshall et al. $[1995$, a] that simpler charge structures were associated with closer proximities to thunderstorm updrafts.

Studies of discharge origin heights by MacGorman et al. [1981], Ray et al. [1987], and Proctor [1991] have described bimodal distributions for the initiation altitudes of intracloud and cloud-to-ground lightning discharges. In Proctor [1991], 773 flashes in South Africa were studied using a time-of-arrival system. The observed distribution of all flash origin heights is shown in the left-hand side of Figure 6.5 (reproduced from Proctor [1991]). Significant peaks in the distribution are evident at heights of 5.3 and $9.2 \mathrm{~km} \mathrm{MSL}$ (ground level for the study was $1.4 \mathrm{~km}$ MSL). The local minimum between the flash origin peaks was centered at $7.4 \mathrm{~km}$ MSL. The average temperatures at the peaks in the altitude distribution were $-3^{\circ}$ and $-28^{\circ} \mathrm{C}$ respectively. These data, along with the observation by Krehbiel et al. [1979] that the charging mechanisms in different locations are the same and generally operate at temperatures colder than $0^{\circ} \mathrm{C}$, indicate that the quiescent region between the two preferential discharge origin heights in the data from South Africa may correspond to the center of the main negative charge region.

The right-hand side of Figure 6.5 shows the distribution of origin heights for 214 cloud-to-ground flashes only. The distribution of origin heights is strikingly similar 

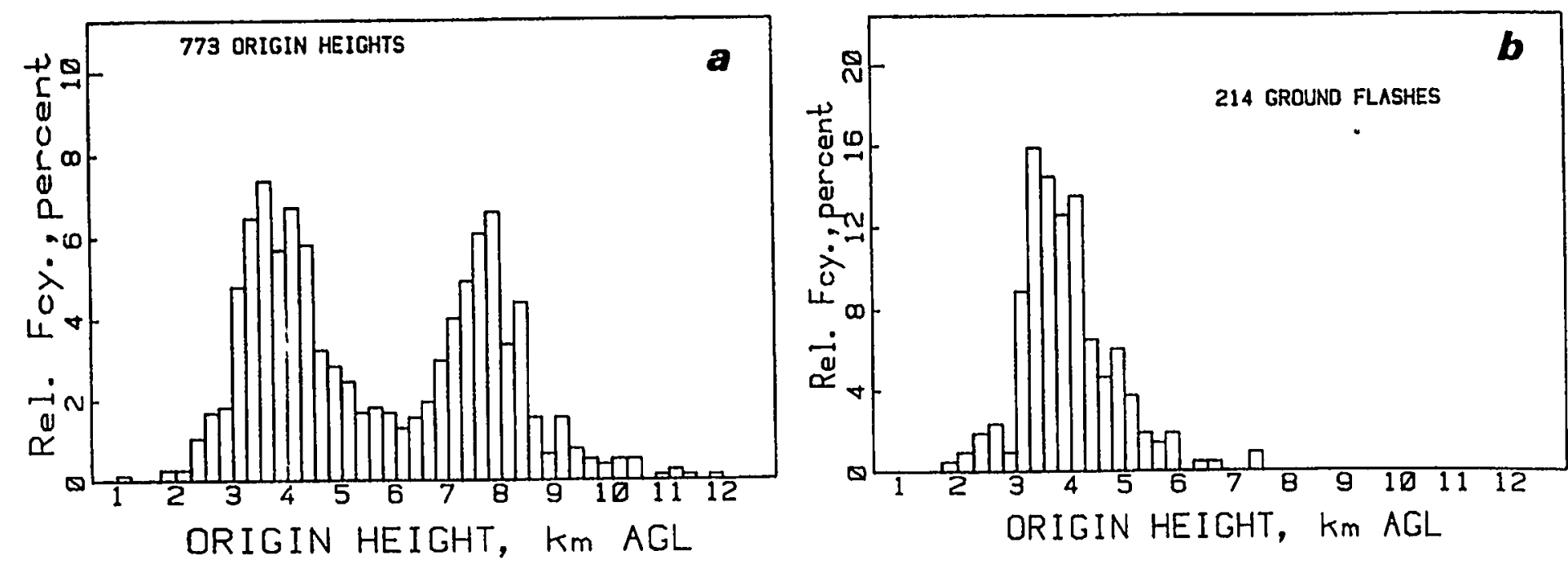

Fig. 1. Distribution of $(a)$ heights above ground level (at $1.43 \mathrm{~km}$ amsl) of the origins of 773 flashes, cloud to ground (CG) and intracloud (IC). that occurred in 13 storms; and (b) origin heights of 214 flashes to ground.

Figure 6.5: Distributions of discharge origin heights for CG and IC strokes (a) and CG strokes only (b) from Proctor [1991]. From D. E. Proctor, Journal of Geophysical Research, 96, p. 5100, 1991, copyright by the American Geophysical Union. 
in mean value and in shape to the lower-altitude feature of the bimodal height distribution shown in the left-hand side of the figure. Proctor [1997] described observations of the discharge heights of 21 intracloud flashes. All flashes in the study originated above $7.4 \mathrm{~km} \mathrm{MSL}$, the altitude corresponding to the local minimum between the two peaks of the author's 1991 study. Proctor's results, which are characterized by a relatively small height uncertainty (on the order of $0.5 \mathrm{~km}$ ), suggest that the bimodal distribution of lightning discharge origin heights results from the fact that intracloud and cloud-to-ground flashes originate at different altitudes within thunderstorms. From the data regarding charge distributions in thunderstorms [Workman et al., 1942; Reynolds and Neill, 1955; Brook et al., 1962; Krehbiel et al., 1974; Jacobson and Krider, 1976; Krehbiel et al., 1979], it is further suggested that sandwiched between the preferential altitudes for IC and CG discharge initiation lies the center of the negative charge region.

Observations from Maier et al. [1996] have provided further evidence that intracloud and cloud-to-ground flashes are initiated at different altitudes within storms. The authors used the Kennedy Space Center LDAR (Lightning Detection and Ranging) system [Lennon and Maier, 1991] to locate initial RF pulses from lightning flashes. A field change meter was used to identify flash types. Cloud-to-ground and intracloud flashes in the study were initiated at heights of 9.5 and $8 \mathrm{~km}$ respectively. The heights were somewhat higher than those reported by Proctor [1991], but this result is consistent with the observation by Jacobson and Krider [1976] that charge altitudes tended to be higher in Florida than in other geographical locations, even after normalization to sea level. In a private communication Paul Krehbiel has stated that 
bimodal flash initiation altitude distributions have often been observed using the KSC LDAR and that the low heights and high heights generally correspond to initial CG and initial IC activity respectively.

The observations are also consistent with those of Shao and Krehbiel [1996], who showed that intracloud flashes often have a bilevel structure between lower negative and upper positive regions of charge. The authors located RF sources using an interferometer and showed that initial breakdown established a vertical channel between the two charge levels and horizontally into the positive charge region. Subsequent breakdown extended the lower-level channel in a retrograde manner horizontally away from the flash origin. The data also indicate that subsequent breakdown tapped regions of negative charge that were lower in altitude than the point of initial breakdown. This observation is consistent with the LDAR data from Maier et al. [1996] and explains why, although initial breakdown altitudes in thunderstorms appear to be bimodal, breakdown locations of whole flashes appear to be more uniform throughout the negative charge region. It would appear that after initial breakdown in both CG and IC strokes, subsequent breakdown progresses further toward the center of the negative charge region.

If this picture is correct, then the CDs described in Chapter 4 occurred at the same altitudes in thunderstorms where intracloud flashes begin, the upper part of the negative charge region. Although the events originated at the same altitudes as intracloud flashes, CDs appeared quite different from typical IC processes, as shown by the comparisons of the electric field change and HF observations presented in this 
chapter and as suggested by calculations of the physical characteristics of the events described in the following chapter. 


\section{CHAPTER 7 \\ CID PHYSICAL CHARACTERISTICS}

The radio emissions from CIDs were described in detail in Chapter 6 and shown to be distinct from the emissions of other thunderstorm electrical processes. Among the outstanding characteristics of the electric field change, HF, and VHF waveforms from CIDs are their short durations, large amplitudes, and temporal isolation from other thunderstorm electrical discharges. Characteristics of the source regions that produced the discharges were also described in Chapter 6 . In the present chapter, the phenomenology of $\mathrm{CID}$ radio emissions is used to determine physical characteristics of the discharges and their source regions.

\subsection{SOURCE ORIENTATION AND RADIATION PATTERN}

The electric field change meters and HF discone antennas that were used to record emissions from CIDs were sensitive only to vertically polarized radiation. For VLF/LF measurements of field and field change, it is not possible to measure horizontal electric fields or electric field changes from the ground because the close proximity of the recording instrumentation to earth forces the tangential or horizontal 
electric field component to be zero. This is the case because the distance between the meters and ground was much less than a wavelength, which ranges from 1 to $100 \mathrm{~km}$ for VLF/LF signals. Thus the field change meters recorded only the vertical component of source electric field change signals. For the case of the HF systems, the antennas were vertically polarized and primarily sensitive to the vertical component of the source electric field for signals that propagated via line-of-sight propagation. Note that scattering could have caused some cross polarization to occur, allowing originally horizontally polarized fields to be sensed by the vertically polarized antenna. For the ionospherically reflected HF signals, no information about the polarization of the source radiation could be determined due to the possibility of Faraday rotation caused by the ionosphere.

The electric field change and HF signals observed from CDs were very powerful. NPBP electric field change pulses had amplitudes that were 0.7 times as large as those from return strokes. The HF electric fields were ten times more powerful than return stroke electric fields. Since both of these measurements were of vertically polarized radiation only, the existence of significant horizontal source radiation components would have meant that the total fields and field changes would have been even larger than those measured. Based on the already very large measured vertical radiation components, it is highly likely that the sources produced primarily vertically polarized radiation. It is certainly true that the sources were not predominately horizontally polarized. Such signals would not have been received by the receiving systems. 
The production of vertically polarized electric field and electric field change signals indicates that charge was transported along a vertically oriented channel. It is thus inferred that the recorded CIDs were vertically oriented discharges. A vertically oriented discharge is consistent with the conclusion presented in the previous chapter that sources occurred between lower negative and upper positive charge regions in the thunderstorms observed. The electric field for such a charge configuration is predominantly vertically oriented. It seems likely that CID breakdown would occur in a direction aligned with the local electric field since the field lines follow the potential gradient. The polarities of the observed field change waveforms also support the conclusion that $\mathrm{CDs}$ occurred between upper positive and lower negative charge regions. All 24 NPBPs were initially positive, indicating that either negative charge was transported upward or positive charge was transported downward. The fact that all of the NPBPs were of the same polarity provides evidence that CIDs were vertically oriented. If field change measurements had been of a randomly oriented or horizontally oriented source, then the polarities of the field change waveforms would have been expected to be random, especially since the observations were not of one storm, but of three storms on three different days. Also, normalized absolute field change amplitudes recorded by the three stations were always in agreement to within $10 \%$. Randomly oriented or horizontally oriented sources would have been likely to produce different amplitudes at the stations, due to stations being further from or closer to the nulls in the radiation pattern. This would have been especially so during storm 3 when FC1 observed events from an azimuth angle that was approximately $90^{\circ}$ from the observation azimuths of stations $\mathrm{FC} 2$ and $\mathrm{FC} 3$. 
A vertically oriented linear discharge would be expected to have a radiation pattern similar to that of a dipole antenna, with nulls in its radiation pattern in upward and downward directions along the axis of the discharge. None of the ground-based observations reported here were made in close proximity to CID discharges, so the source radiation pattern was never probed in the null regions.

\subsection{SIZE, CHARGE, AND CURRENT}

A distinct characteristic of the radio emissions from $\mathrm{CDs}$ was the short time scale on which the events took place. The HF and VHF radio emissions from the sources typically lasted between 3 and $4 \mu$ s. NPBP electric field change emissions from the source lasted for $25 \mu \mathrm{s}$, with the initial portion of the bipolar pulse lasting less than $5 \mu \mathrm{s}$. A brief time scale indicates that the sources that produced the emissions were compact. Characteristics of the RF and electric field change emissions described in the previous chapter provide insight into the size of CDs and the physical processes that actually take place.

It has been previously been observed that detectable HF and VHF radiation from thunderstorms is produced primarily by breakdown processes and not by charge flow along already conducting channels [Rhodes et al., 1994; Shao et al., 1995]. This result is exemplified by the absence of detectable $\mathrm{RF}$ radiation during the initial parts of return strokes initiated by dart leaders and during long intervals of continuing current discharges to ground. Thus, events that produce powerful $\mathrm{RF}$ radiation do not 
necessarily produce large field change signals and events that produce large field change signals do not necessarily produce powerful RF radiation. Whereas RF is produced by breakdown processes, field change signals are produced by charge transfer processes.

When $\mathrm{HF}$ and electric field change emissions from CIDs were recorded simultaneously from a single location, the arrival of the broadband HF emissions slightly preceded the arrival of the electric field change pulse. Figure 7.1 shows $50 \mu$ s records of the $\mathrm{HF}$ and field change emissions recorded by $\mathrm{HF} 1$ and $\mathrm{FC} 1$ from a $\mathrm{CDD}$ on the night of 17 August 1996. Longer waveform records from the same event were shown in Figure 4.1. In Figure 7.1, the centroid time of the HF radiation occurs $5 \mu \mathrm{s}$ prior to the peak amplitude of the field change signal and $9 \mu$ s prior to the zero crossing of the field change signal. The NPBP zero crossing is indicative of the time of peak current in the observed charge transfer process. For 10 of the events for which broadband $\mathrm{HF}$ and electric field change records were received, the $\mathrm{HF}$ centroid time preceded the NPBP zero crossing time by a mean delay of $8 \mu \mathrm{s}$. Part of this delay may be due to the propagation effects described in Chapter 6, but this part is not more than $2 \mu \mathrm{s}$. So, on average, the RF bursts occurred $6 \mu$ s before the time of peak current in the charge transfer process. This timing relationship is not unusual. Normal intracloud pulses recorded by $\mathrm{HF} 1$ and $\mathrm{FCl}$ exhibited similar delays. The observations are consistent with the results of Krider et al. [1979] who, in their observations of intracloud lightning discharges, found "a clear tendency for the RF to peak during the initial half cycle of the field." 

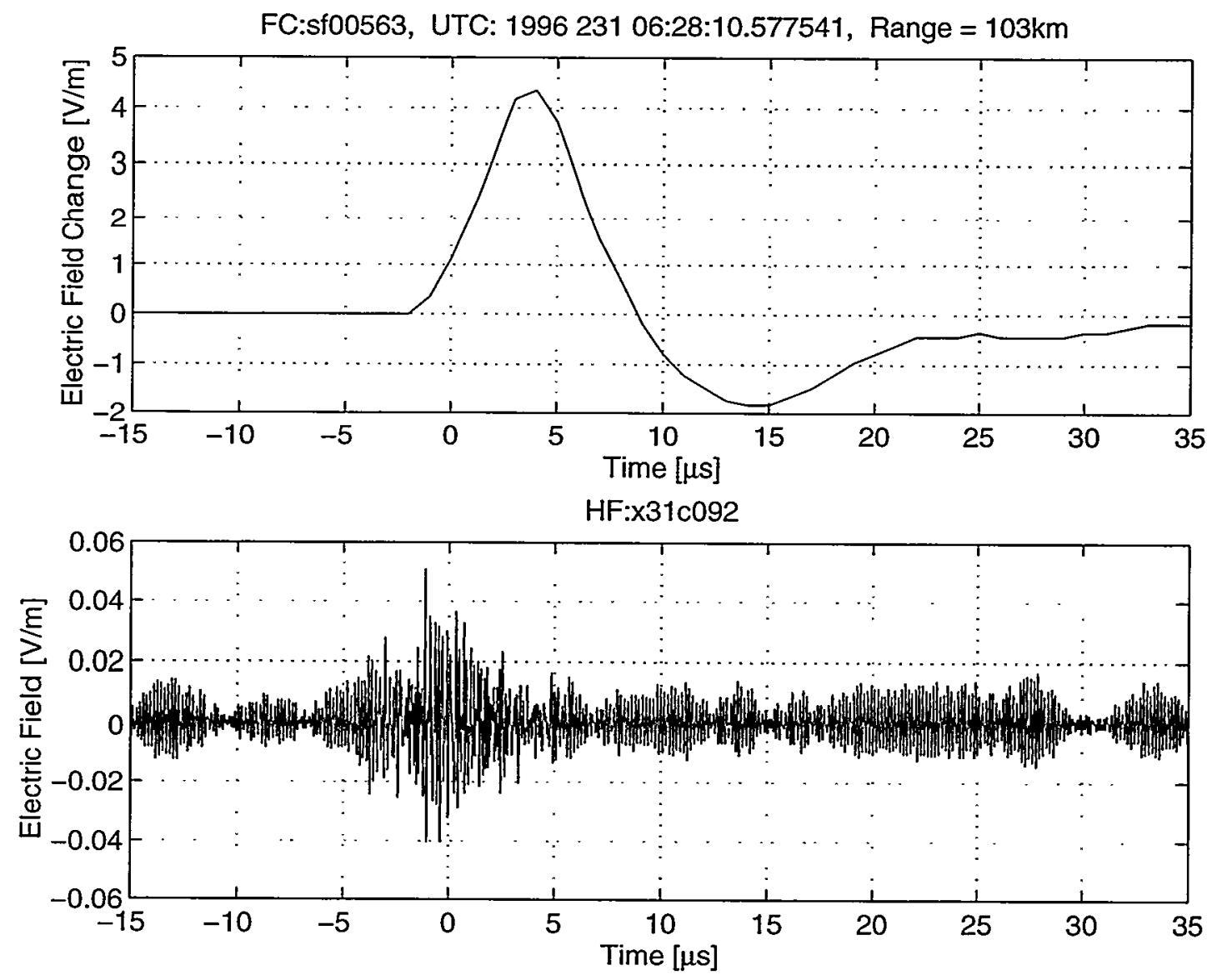

Figure 7.1: Illustration of the short-time-scale relationship between $\mathrm{CID}$ electric field change and broadband HF emissions. The upper panel shows the field change waveform. The lower panel shows the HF waveform. The HF radiation precedes the gross field change. 
The relative timing between the $\mathrm{RF}$ emissions and field change emissions suggests that the RF radiation is emitted during a breakdown process that forms a current-carrying channel. Subsequent charge motion along the channel produces the bipolar electric field change pulses.

The length of the channel can be calculated from the duration of the HF emissions if the velocity with which the channel is formed is known. Streak camera observations of thunderstorms by Orville and Idone [1982] showed that the breakdown of virgin air in lightning stepped leaders occurred at a speed on the order of $5 \times 10^{7} \mathrm{~m} / \mathrm{s}$. This velocity was sustained for a distance of around $50 \mathrm{~m}$ and a time of approximately one microsecond during each leader step. Note that pauses between steps cause the average speed of a leader to be much slower than its peak velocity. Most measurements of average 2-D leader velocities have been between 1 and $2 \times 10^{5}$ $\mathrm{m} / \mathrm{s}$ [Uman, 1987, a]. The temporal isolation of CIDs suggests that initial breakdown in the events also occurs in virgin air. Thus an initial streamer velocity estimate of 5 $\times 10^{7} \mathrm{~m} / \mathrm{s}$ is reasonable and can be used to determine the spatial scale of the CID with Equation 7.1:

$$
\Delta z=v \times \Delta t
$$

The median duration of the HF radio burst from a CD was $3.2 \mu \mathrm{s}$. If it is assumed that the associated breakdown occurs at a velocity of $5 \times 10^{7} \mathrm{~m} / \mathrm{s}$ then the spatial scale of the discharge is $160 \mathrm{~m}$. Table 7.1 shows this result, along with other 
calculated properties of CID discharges for velocities varying from one thirtieth to one times the speed of light.

\begin{tabular}{|l|l|l|l|l|}
\hline Velocity $(v)$ & $1 \times 10^{7} \mathrm{~m} / \mathrm{s}$ & $5 \times 10^{7} \mathrm{~m} / \mathrm{s}$ & $1 \times 10^{8} \mathrm{~m} / \mathrm{s}$ & $3 \times 10^{8} \mathrm{~m} / \mathrm{s}$ \\
\hline Scale Size $(\Delta z)$ & $32 \mathrm{~m}$ & $160 \mathrm{~m}$ & $320 \mathrm{~m}$ & $960 \mathrm{~m}$ \\
Charge Transferred $(\Delta Q)$ & $16 \mathrm{C}$ & $3.1 \mathrm{C}$ & $1.6 \mathrm{C}$ & $0.52 \mathrm{C}$ \\
Average Current $\left(i_{\text {avg }}\right)$ & $1000 \mathrm{kA}$ & $210 \mathrm{kA}$ & $100 \mathrm{kA}$ & $35 \mathrm{kA}$ \\
Charge Density $(\rho)$ & $932,000 \mathrm{nC}^{3} \mathrm{~m}^{3}$ & $1400 \mathrm{nC} / \mathrm{m}^{3}$ & $93 \mathrm{nC} / \mathrm{m}^{3}$ & $1.1 \mathrm{nC}^{3}$ \\
Peak E-Field $\left(E_{\text {max }}\right)$ & $1100 \times 10^{6} \mathrm{~V} / \mathrm{m}$ & $8.7 \times 10^{6} \mathrm{~V} / \mathrm{m}$ & $1.1 \times 10^{6} \mathrm{~V} / \mathrm{m}$ & $0.040 \times 10^{6} \mathrm{~V} / \mathrm{m}$ \\
\hline
\end{tabular}

Table 7.1: Scale size, charge transferred, and average current for CIDs as a function of streamer propagation velocity. Values for charge density and peak electric field are also given, as inferred from the model presented in Section 7.3.

In Section 7.1 it was shown that recorded CIDs were vertically oriented and that NPBP polarities were consistent with the motion of negative charge upward or positive charge downward. Willett et al. [1989] and Medelius et al. [1991] observed electric field change waveforms of the opposite polarity (NNBPs), indicating that the relative charge positions were reversed.

It was concluded in Chapter 6 that CID discharge altitudes were consistent with an origin between lower negative and upper positive charge regions. Previous studies of thunderstorms in New Mexico have shown that the main negative charge region is 
usually between 6 and $8 \mathrm{~km}$ MSL [Workman et al., 1942; Reynolds and Neill, 1955; Brook et al., 1962; Krehbiel et al., 1974] with positive charge overhead. CID heights were within the range of 8 to $11 \mathrm{~km} \mathrm{MSL}$ for the three southwestern thunderstorms observed. The source heights indicate that the events occurred between the main negative and upper positive thunderstorm charge regions.

Balloon observations of electric fields have shown that in many storms, a simple positive dipole model for thunderstorm charge distribution is not sufficient and that many stratified charge regions (up to ten) can exist [Marshall and Rust, 1991; Marshall et al., 1995, a]. Perhaps the NNBP-producing discharges recorded by Willett et al. [1989] and Medelius et al. [1991] occurred within such a charge structure between regions of underlying positive charge and overlying negative charge.

Thus the picture, so far, is that a vertical breakdown discharge occurs between a lower negative charge region and upper positive charge region. The length of the discharge is between many tens and many hundreds of meters, based on assumed propagation velocities between one thirtieth and one times the speed of light (see Table 7.1). The characteristics of the breakdown discharge are an interesting point of speculation. Based on the observations of Rhodes et al. [1994] and Shao et al. [1995], most radio frequency radiation from lightning is produced by negativepolarity breakdown, that is, by breakdown that transports negative charge in its direction of progression. Based on their results and the fact that the RF radiation from CIDs is very powerful ( $20 \mathrm{~dB}$ brighter than that produced by other lightning breakdown processes), it might be concluded that the strongly radiating part of the 
streamer results from negative breakdown progressing in an upward direction and transporting negative charge upward. On the other hand, it was also concluded by Shao et al. [1995] that positive breakdown that produces a conducting channel is difficult to initiate. As will be shown shortly, electric field strengths just prior to CIDs are comparable to or greater than the highest electric fields that have been measured within thunderstorms. If the initiation of negative-polarity breakdown is somehow inhibited by the electrical and/or physical conditions that exist prior to the occurrence of CIDs, then positive-polarity breakdown at an elevated level of electrical stress may occur. Whether positive-polarity breakdown produces powerful or even detectable $R F$ radiation has been a subject of much debate [Mazur, 1989]. However, the observations of Shao et al. [1995] show that positive breakdown events are observed that radiate strongly at radio frequencies. Propagation speeds of positivepolarity streamers were estimated by Shao et al. [1995] to fall in the range from $1 \times$ $10^{7}$ to $6 \times 10^{7} \mathrm{~m} / \mathrm{s}$. Such velocities are within the range of values used to compute streamer lengths for CIDs in Table 7.1. Whether positive- or negative-polarity breakdown occurs in CIDs or even whether a bi-directional breakdown process occurs cannot be determined from the measurements presented here.

Once a conducting channel has been formed by the RF-emitting breakdown process, current can flow along the channel. Refer again to Figure 7.1 for an illustration that the electric field change signal, indicating charge transfer, rises as the RF signal ceases. The close proximity of the electric field change meters to the earth meant that they were sensitive only to changes in the vertical component of the electric field. Because CIDs are vertically oriented discharges, the events probably do 
not produce large horizontal field changes components and the field change meters are essentially sensitive to the entire electric field change signal.

From CID electric field change waveforms, the dipole moment change associated with the events can be calculated. Electric field change $(\Delta E)$ can be determined from dipole moment change $(\Delta p)$ using the following relationship (adapted from Uman [1984]):

$$
\Delta E=\frac{1}{4 \pi \varepsilon_{o}}\left[\frac{1}{r^{3}}\left(\frac{\Delta p}{1}\right)+\frac{1}{c r^{2}}\left(\frac{d \Delta p}{d t}\right)+\frac{1}{c^{2} r}\left(\frac{d^{2} \Delta p}{d t^{2}}\right)\right]
$$

where $\varepsilon_{o}$ is the permittivity of free space and $r$ is the range from the source.

The three bracketed terms on the right-hand side of Equation 7.2 are the electrostatic, induction, and radiation terms of the total field respectively. Because all observations of CIDs were made from distances of greater than $80 \mathrm{~km}$, the electrostatic and inductive terms, which are inversely proportional to $r^{3}$ and $r^{2}$ respectively, were set to zero. Eliminating the terms and rearranging the equation, the following relationship between dipole moment change and electric field change remains:

$$
\Delta p=4 \pi \varepsilon_{o} c^{2} r \iint \Delta E d t d t
$$


Equation 7.3 was used to determine dipole moment change values (10 to 90\%) associated with 15 CID NPBP waveforms that were not saturated. The mean value was $0.38 \mathrm{C}-\mathrm{km}$. The minimum and maximum values were 0.26 and $0.80 \mathrm{C}-\mathrm{km}$. Nine NPBPs that were not included in the 15 used to compute the mean dipole moment change saturated the electric field change digitizers. A few of these events would have yielded dipole moment change values in excess of $1.0 \mathrm{C}-\mathrm{km}$. Taking into account the estimated dipole moment changes associated with the saturated waveforms, a value of $0.5 \mathrm{C}-\mathrm{km}$ will be assumed as typical for $\mathrm{CDD}$ discharges. This value, in itself, is not remarkable compared to other thunderstorm electrical discharges, it is the rate of dipole moment change, also known as current moment, that is remarkable in comparison to other cloud processes. A typical cloud-to-ground return stroke produces a dipole moment change on the order of a few hundred $\mathrm{C}-\mathrm{km}$ [Brook et al., 1962; Jacobson and Krider, 1976]. Cloud flash dipole moment changes are on the order of a few tens to a few hundred C-km [Wang, 1963; Brook and Ogawa, 1977]. Individual K-change dipole moment changes are on the order of a few C-km [Brook and Ogawa, 1977; Ogawa and Brook, 1964]. Thus CD dipole moment changes are non-spectacular compared to other lightning processes. Refer to Table 7.2 for a comparison of the physical characteristics of CIDs to those of other lightning processes.

Current moments for CID can be calculated from the time it takes for the dipole moment changes to occur. The mean dipole moment change duration (10 to 90\%) for the 15 unsaturated NPBPs was $13.7 \mu$ s. An estimated duration including the 9 saturated waveforms is $15 \mu \mathrm{s}$. A $0.5 \mathrm{C}-\mathrm{km}$ dipole moment change that occurs in 15 
$\mu$ s produces a current moment of $33 \mathrm{C}-\mathrm{km} / \mathrm{ms}$. This value is ten times that of $\mathrm{K}$ change current moments computed from results in Brook and Ogawa [1977] and Krehbiel [1981].

\begin{tabular}{|l|l|l|l|l|l|}
\hline & CID & K-change & Stepped Leader Step & IC Flash & Negative CG \\
\hline$\Delta t$ & $15 \mu \mathrm{s}$ & $2 \mathrm{~ms}$ & $50 \mu \mathrm{s}$ & $500 \mathrm{~ms}$ & $50 \mu \mathrm{s}$ \\
$\Delta z$ & $160 \mathrm{~m}$ & $2 \mathrm{~km}$ & $50 \mathrm{~m}$ & $3 \mathrm{~km}$ & $5 \mathrm{~km}$ \\
$\nu$ & $5 \times 10^{7} \mathrm{~m} / \mathrm{s}$ & $2 \times 10^{6} \mathrm{~m} / \mathrm{s}$ & $5 \times 10^{7} \mathrm{~m} / \mathrm{s}$ (peak) & $3 \times 10^{5} \mathrm{~m} / \mathrm{s}$ & $1 \times 10^{7} \mathrm{~m} / \mathrm{s}$ \\
& & $3 \times 10^{5} \mathrm{~m} / \mathrm{s}$ (avg.) & & \\
$\Delta p$ & $0.5 \mathrm{C}-\mathrm{km}$ & $7 \mathrm{C}-\mathrm{km}$ & $0.003 \mathrm{C}-\mathrm{km}$ & $90 \mathrm{C}-\mathrm{km}$ & $25 \mathrm{C}-\mathrm{km}$ \\
$\Delta p / \Delta t$ & $33 \mathrm{C}-\mathrm{km} / \mathrm{ms}$ & $3.5 \mathrm{C}-\mathrm{km} / \mathrm{ms}$ & $50 \mathrm{C}-\mathrm{km} / \mathrm{ms}$ & $0.2 \mathrm{C}-\mathrm{km} / \mathrm{ms}$ & $500 \mathrm{C}-\mathrm{km} / \mathrm{ms}$ \\
$\Delta Q$ & $3.1 \mathrm{C}$ & $3.5 \mathrm{C}$ & $0.05 \mathrm{C}$ & $30 \mathrm{C}$ & $5 \mathrm{C}$ \\
$i_{\text {avg }}$ & $210 \mathrm{kA}$ & $2 \mathrm{kA}$ & $1 \mathrm{kA}$ & $3 \mathrm{kA}$ & $30 \mathrm{kA}$ \\
\hline
\end{tabular}

Table 7.2: A comparison of the physical characteristics of CIDs versus representative values for other types of lightning processes. Data taken from Reynolds and Neill [1955], Ogawa and Brook [1964], Brook and Ogawa [1977], Krehbiel [1981], Idone and Orville [1982], Orville and Idone [1982], and Uman [1987, a, b].

Using the typical CID dipole moment change value of $0.5 \mathrm{C}-\mathrm{km}$ and the previously estimated breakdown streamer length of $160 \mathrm{~m}$, the effective charge transferred along the conducting channel can be computed from the following relation: 


$$
\Delta Q=\frac{\Delta p}{\Delta z}
$$

The computed charge is $3.1 \mathrm{C}$. The average current that is produced by moving $3.1 \mathrm{C}$ in $15 \mu \mathrm{s}$ is $210 \mathrm{kA}$ as computed from the following equation:

$$
i_{\text {avg }}=\frac{\Delta Q}{\Delta t}
$$

Refer to Table 7.1 to see how these computed values change for different assumed propagation velocities of the initial breakdown streamer. Refer to Table 7.2 for a comparison of the computed charge and average current values to those observed for other lightning processes. An average current of $210 \mathrm{kA}$ is quite large for an isolated breakdown event. Inferred average currents during stepped leader processes are estimated to be from one to several kA [Uman, 1987, a], although the low duty cycle of leaders indicates that average currents during steps may be appreciably higher. The typical peak current associated with negative cloud-toground return strokes is 20 to $40 \mathrm{kA}$ with $1 \%$ of events producing peak currents in excess of 200 kA [Berger et al., 1975]. Positive CGs produce a typical peak current of $35 \mathrm{kA}$ with $5 \%$ of events producing peak currents greater than $250 \mathrm{kA}$ [Berger et al., 1975].

The computations described above were made using an assumed breakdown streamer velocity of $5 \times 10^{7} \mathrm{~m} / \mathrm{s}$. This value was reported by Orville and Idone 
[1982] as the speed at which leader steps break down virgin air. Table 7.1 and Figure

7.2 show how the computed values for discharge length, charge, and average current change for different assumed velocities. The values for average current in Table 7.1 suggest that the most reasonable velocity estimates are probably within the upper portion of the range from $1 \times 10^{7}$ to $3 \times 10^{8} \mathrm{~m} / \mathrm{s}$, since currents greater than $200 \mathrm{kA}$ are rarely observed, except in occasional association with positive cloud-to-ground return strokes. Reports of currents as large as $500 \mathrm{kA}$ from any lightning process are absent from the literature. For the calculations in the following section, values from the third column of Table 7.1 will be used in a model to determine charge density and peak electric field. The values from column three are $v=1 \times 10^{8} \mathrm{~m} / \mathrm{s}, \Delta z=320 \mathrm{~m}$, $\Delta Q=1.6 \mathrm{C}$, and $i_{\text {avg }}=100 \mathrm{kA}$.

\subsection{Electric Fields and Charge Densities PRior to CDS}

By making an assumption about the distribution of charge prior to a $\mathrm{CD}$, it is possible to compute the electric fields and charge densities that were present. As a simple model, assume that two spherical regions of charge with equal and uniform charge densities are neutralized during a $\mathrm{CD}$. Such a configuration is pictured in

Figure 7.3. The separation between the charge centers is $\Delta z$ (as in the previous section). The radius of each sphere is $\Delta z / 2$. The charge density ( $\rho$ ) of each region is given by the total charge in each sphere divided by its volume: 


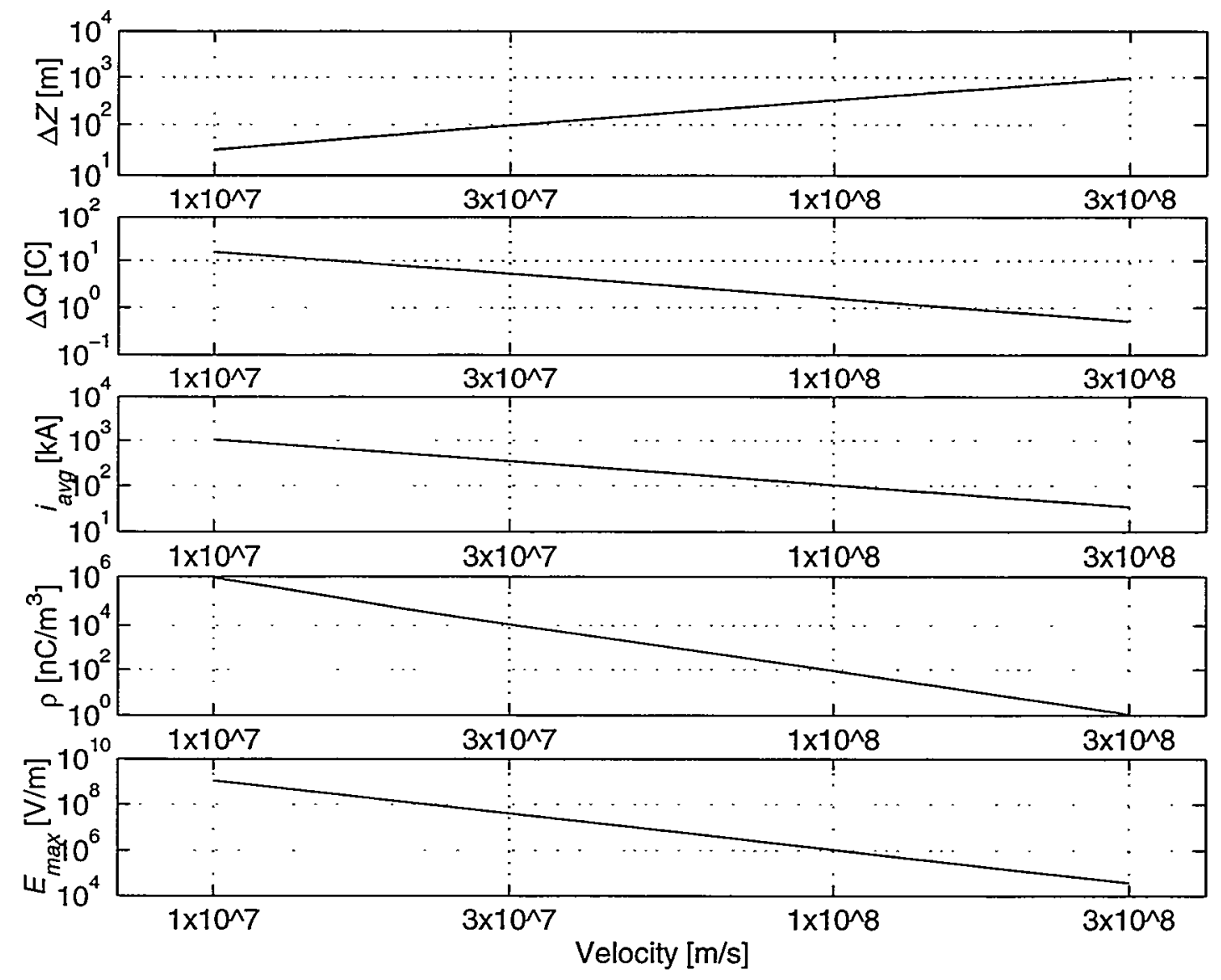

Figure 7.2: $\quad$ Plots of spatial extent $(\Delta Z)$, charge $(\Delta Q)$, average current $\left(i_{\text {avg }}\right)$, charge density $(\rho)$, and peak electric field $\left(E_{\max }\right)$ as a function of the assumed streamer propagation velocity $(v)$. 


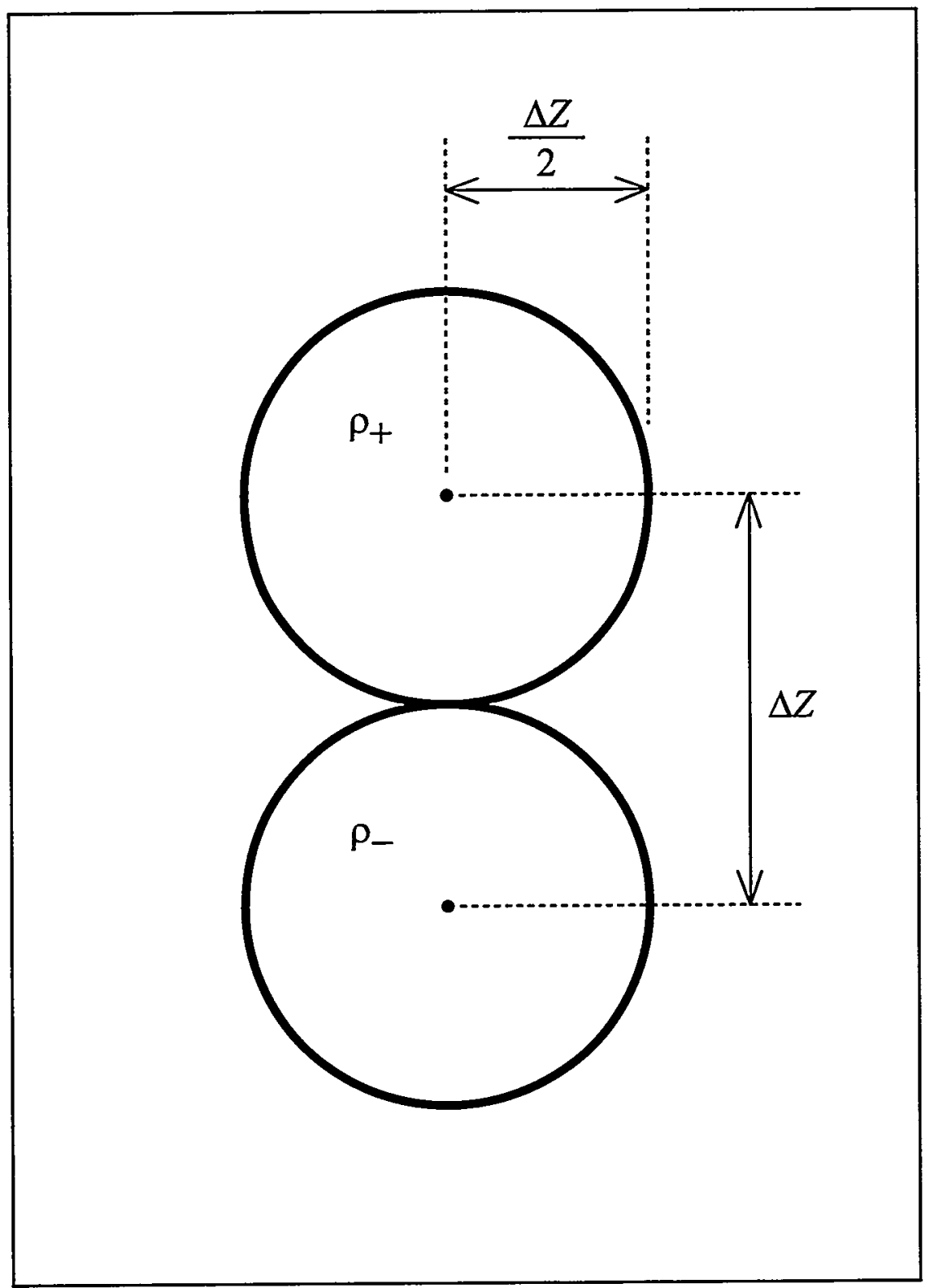

Figure 7.3: Assumed charge configuration for the calculation of charge densities and peak electric fields. Spherical charge regions with constant charge densities $(\rho)$ were assumed. The separation between the regions was $\Delta Z$. The radii of the regions were $\Delta Z / 2$. 


$$
\rho=\frac{\Delta Q}{\frac{4}{3} \pi\left(\frac{\Delta z}{2}\right)^{3}}
$$

For the case of $\Delta z=320 \mathrm{~m}$ that was explored in the previous section, $\Delta Q$ was 1.6 C, so $\rho$ works out to $93 \mathrm{nC} / \mathrm{m}^{3}$. This charge density is over an order of magnitude greater than charge densities inferred from in situ measurements of thunderstorm electric fields when a 1-D approximation to Gauss's law is used [Marshall and Rust, 1991; Stolzenburg and Marshall, 1994; Marshall et al., 1995, a]. The unique character of CIDs may require such an extreme charge density.

For the simple geometry assumed here, the maximum electric field value exists at the midpoint between the two charge centers. The electric field $\left(E_{\max }\right)$ at that point is given by:

$$
E_{\max }=\frac{\Delta Q}{4 \pi \varepsilon_{o}}\left[\frac{2}{(\Delta z / 2)^{2}}\right]
$$

For the values of $\Delta Q$ and $\Delta z$ given previously in this section, $E_{\max }$ works out to $1.1 \times 10^{6} \mathrm{~V} / \mathrm{m}$. Measurements of electric field strengths inside active storms with instrumented rockets and balloons have shown that electric field strengths are usually in the range of $10^{4}$ to $10^{5} \mathrm{~V} / \mathrm{m}$, with typical peak values of 1.5 to $2 \times 10^{5} \mathrm{~V} / \mathrm{m}$ [Winn et al., 1974; Marshall and Rust, 1991; Marshall et al., 1995, a]. Winn et al. [1974] 
obtained one measurement of $4 \times 10^{5} \mathrm{~V} / \mathrm{m}$. Refer to Table 7.1 and Figure 7.2 to see how $\rho$ and $E_{\max }$ change with the other parameters introduced in Section 7.2

The intermittent occurrence of CID discharges, coupled with their energetic and unusual nature, suggests that they occur when storms develop localized regions of - very strong electric fields. The observations of tropical cyclone Fausto in Chapter 5 showed that it may be possible to regenerate the conditions very quickly (within several seconds), or that multiple CID-producing regions may exist in a storm. Fausto produced CIDs in a rhythmic fashion every 8 seconds or so for at least 16 minutes. The strong field values that are required could be the 1 to $2 \times 10^{5} \mathrm{~V} / \mathrm{m}$ values that have often been measured or even stronger values, up to $10^{6} \mathrm{~V} / \mathrm{m}$ or greater. The field change would be the difference of the electric field prior to and after the discharge and could potentially be stronger than the initial field.

For any combination of $v, \Delta z, \Delta Q, i_{\text {avg }}, \rho$, and $E_{\max }$, the physical characteristics of the discharge are unlike those for any type of previously observed lightning process. This is shown by the scatter plot in Figure 7.4, which shows ranges of average current versus discharge length for the processes listed in Table 7.2. CIDs stand alone as discharges that produce currents as high or higher than those produced by return strokes, but which are much shorter in duration and spatial extent. 


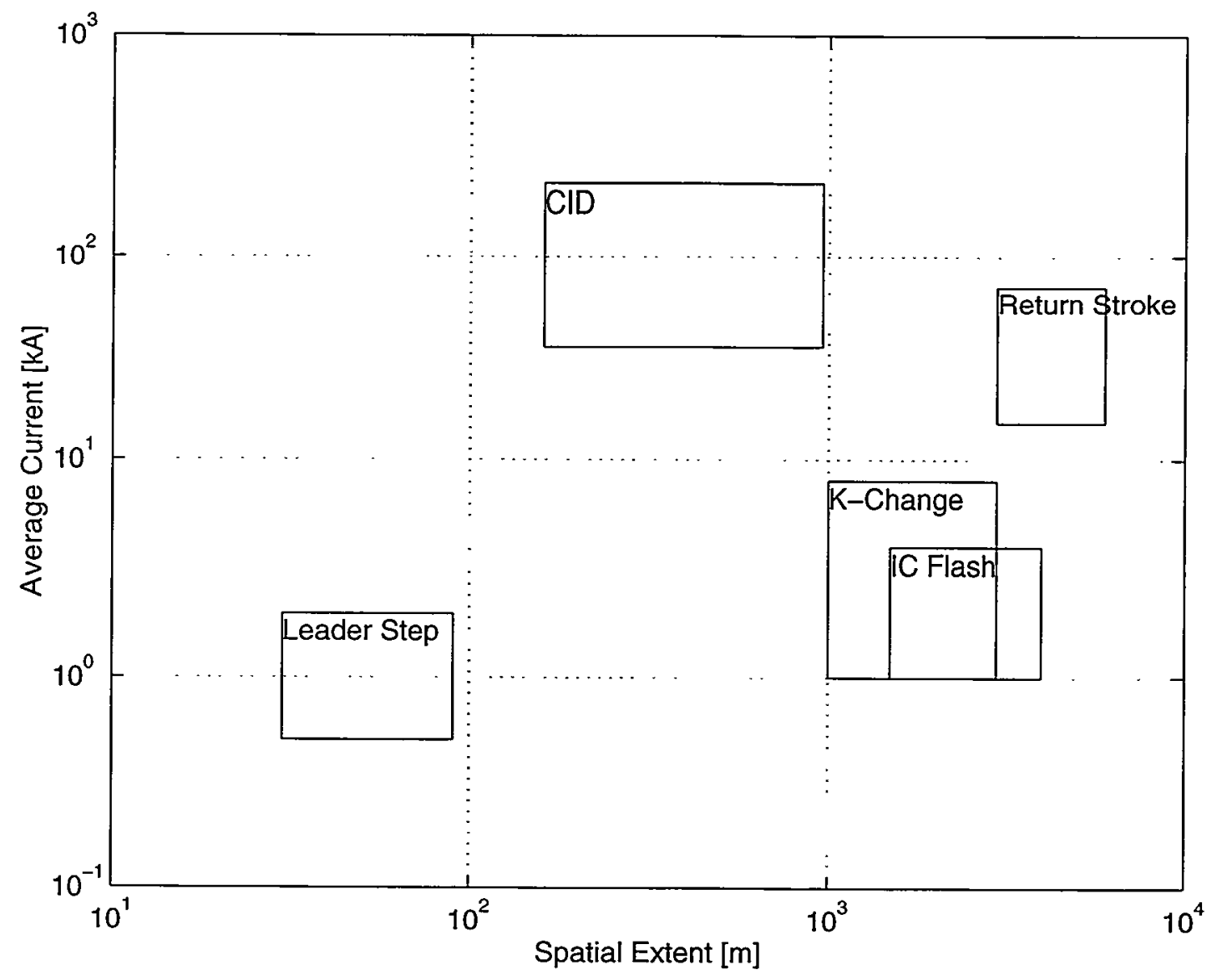

Figure 7.4: $\quad$ Scatter plot of average current $\left(i_{a v g}\right)$ versus spatial extent $(\Delta Z)$ for CIDs and other thunderstorm electrical processes. 


\section{CHAPTER 8}

\section{CONCLUSION}

A very compact source of distinct thunderstorm radio emissions has been identified and characterized. The source produces unique broadband radio signals from VLF to UHF. Depending on the band being observed, the signals from these discharges have previously been referred to in the literature as narrow positive or narrow negative bipolar pulses (NPBPs or NNBPs, observed at VLF/LF), subionospheric pulse pairs (SIPPs, observed at HF), or transionospheric pulses pairs (TIPPs, observed at VHF). In Chapter 1 of this dissertation, the sources of these unique broadband radio emissions were dubbed CIDs, an acronym for compact intracloud discharges. CIDs are intracloud electrical phenomena that occur within a source region that is between a few hundred meters and one kilometer in spatial extent. They occur in areas of intense thunderstorm activity as indicated by measurements of radar reflectivity and other lightning activity. It is concluded that CDs are naturally occurring events and do not occur as a result of anthropogenic influence. They cannot be produced by power line discharges because all measured source heights were above $8 \mathrm{~km}$ MSL. They cannot be discharges from aircraft because events occurred within or in very close proximity to $50 \mathrm{dBZ}$ radar reflectivity echoes. Aircraft avoid such areas of intense thunderstorm activity. 
TIPPs are the powerful, paired, broadband VHF radio emissions from CIDs that have been observed by the Blackbeard satellite instrument. SIPPs are also paired radio emissions from $\mathrm{CIDs}$, but have been observed in the HF radio spectrum using broadband ground-based receivers. TIPP/SIPP second pulses result from the reflection of CD RF radiation from the surface of the earth. This conclusion is supported by measurements of surface reflectivity in the VHF and verification that CIDs have a broad radiation pattern. Also, delays between the direct and reflected signals were entirely consistent with the heights of recorded sources and the locations from which the pulse pairs were observed. The median durations of TIPP RF pulses were between 3.2 and $3.8 \mu$ s as measured by both Blackbeard and the ground-based broadband HF receivers. Measurements from both resources have shown that the HF/VHF emissions from CIDs are $20 \mathrm{~dB}$ more powerful than those emitted by normal intracloud and cloud-to-ground lightning streamer processes. Unlike the emissions from other lightning processes that were observed, CID RF pulses occurred in isolation. In all $24 \mathrm{HF}$ recordings of CIDs, the single event was the only transient discharge within the record (HF records were one millisecond in duration).

NPBPs are the VLF/LF electric field change emissions from CIDs. All CID field change waveforms were bipolar with an initially-positive polarity, indicating that either negative charge was moved upward or positive charge was moved downward. Similar pulses of an initially-negative polarity (NNBPs) were not observed (perhaps due in part to the configuration of the triggering system), but such events have been observed by other researchers. The mean FWHM duration for the initial bipolar field change pulse was $4.7 \mu \mathrm{s}$. The mean duration for the entire NPBP was $25 \mu \mathrm{s}$. 
Propagation effects shifted these values toward longer durations than would have been recorded closer to the source. The peak amplitudes of NPBP field change records were on average 0.7 times those from negative cloud-to-ground return strokes and 2.6 times those from detected intracloud pulses. Unlike the field change waveforms from all other lightning processes that were observed, NPBP pulses usually occurred in isolation from other thunderstorm transients. In $71 \%$ of NPBP records, the bipolar pulse, with its ionospheric reflections, was the only event within the record. In the records that did contain other lightning activity, the additional field change signals were produced by intracloud events that occurred after the NPBP. It is thus suggested that CIDs usually occur in isolation, but may initiate normal intracloud lightning discharges some fraction of the time.

Twenty-four CIDs were recorded from three nighttime airmass thunderstorms that occurred in the southwestern US. The calculated altitudes of the discharges ranged from 8 to $11 \mathrm{~km} \mathrm{MSL}$. Uncertainties in the height estimates were between \pm 0.3 and $0.8 \mathrm{~km}$. Based on the measurements of other researchers, this range of altitudes corresponds to an area above the main negative charge region for thunderstorms of the type observed. The calculated source heights for over 100 events that were recorded from tropical cyclone Fausto were between 15 and $17 \mathrm{~km}$ MSL. The uncertainty in these measurements was $\pm 2 \mathrm{~km}$. Observations of source heights greater than $15 \mathrm{~km}$ were consistent with Blackbeard data.

Determinations of $\mathrm{CD}$ locations in latitude and longitude showed that they occurred in areas of intense cloud-to-ground lightning activity (between 2 and 18 ground flashes per minute) as reported by the National Lightning Detection Network. 
Radar reflectivity data from two of the storms showed that CIDs occurred within or in very close proximity to areas that produced peak radar reflectivities of between 47 and $58 \mathrm{dBZ}$. Bulk radar reflectivities in these areas were greater than $40 \mathrm{dBZ}$. For one of the storms, which had a $0.4 \mathrm{~km}$ by $8.0 \mathrm{~km}$ location uncertainty ellipse, the high reflectivity region (which was $2 \mathrm{~km}$ across) was centered on the locations of eight CIDs that occurred during a span of 40 minutes. For the other storm, the location uncertainties were much larger, but consistent with the sources occurring in the high reflectivity thunderstorm core. Although bright radar echoes were associated with CID source regions, it has yet to be determined whether high radar reflectivity characterizes all CID source regions or all high reflectivity regions produce CID discharges.

Based on analysis of $\mathrm{CID}$ waveforms, the discharges are 300 to $1000 \mathrm{~m}$ in spatial extent. The initial breakdown that forms the conducting channel proceeds at a speed that is between one tenth and one times the speed of light. Dipole moment change calculations indicate that between 0.5 and $2.0 \mathrm{C}$ of charge are transported during a $\mathrm{CID}$, producing an average current of between 30 and $200 \mathrm{kA}$ for $15 \mu \mathrm{s}$. A model of the charge distribution in the CID source region prior to discharge initiation indicated that charge densities were many tens of $\mathrm{nC}^{3} \mathrm{~m}^{3}$ and electric field strengths were in excess of $1 \times 10^{6} \mathrm{~V} / \mathrm{m}$. Both of these values are an order of magnitude higher than values previously reported in the literature.

CIDs are vertically oriented discharges. Some evidence of this preferential orientation was provided by the fact that all 24 NPBPs were of an initially-positive polarity. If a horizontal or random orientation is assumed, then random polarities are 
expected. The polarities of the discharges and their altitudes within New Mexico thunderstorms were consistent with the events occurring between lower negative and upper positive charge regions. Other researchers have observed NNBPs, pulses similar to NPBPs, but of an initially-negative polarity. Such pulses could be produced by the same mechanism that produces NPBPs, but under conditions such that the electric field polarity is reversed. This could happen between layers of lower positive and upper negative charge. Within such a charge structure in a given storm, it is predicted that NPBPs and NNBPs would be produced at different altitudes within the storm. Synoptic studies of many thunderstorms might also yield preferential altitudes for the origins of NPBPs and NNBPs. The CIDs that were associated with tropical cyclone Fausto occurred at much higher altitudes than the southwestern US CIDs (15 $-17 \mathrm{~km}$ versus $8-11 \mathrm{~km}$ ). The electric field change polarities of the Fausto events were not known. It is possible that the events were upside-down CDs that produced NNBPs, possibly explaining their higher altitude origins.

Unlike all other lightning processes that were observed, CDs occurred as single, isolated discharges. The mechanism for the production of singular discharges is not known, but there appears to be some sort of minimum criterion for its occurrence. Many storms that were observed during the summer of 1996 did not produce NPBPs. When the events were observed, they occurred in clusters, as if a threshold had been crossed. Once the minimum conditions were set up, many storms produced NPBPs in succession with inter-event time separations that ranged from seconds to minutes. Tropical cyclone Fausto was the best example of this thunderstorm "metronome," producing CIDs every eight seconds, on average, for the sixteen minutes during which 
data were acquired. The minimal condition required for CDs to occur may be an electric field threshold, a meteorological condition related to the charge separation process, or some other geophysical criterion. The New Mexico data suggest that the condition is correlated with high radar reflectivity, since reflectivity values of 40 to $58 \mathrm{dBZ}$ were observed in conjunction with the discharges. If this is so, then CDs may occur only in the intense cores of thunderstorm, in regions of locally elevated electric field amplitudes. Such a scenario could explain the lack of previous observations of the charge densities and electric field values calculated here. If the source region is spatially small, like the high reflectivity regions observed in the New Mexico storms (one or two km across), and is present only some of the time, then it may be that in situ measurement devices have yet to probe this region of thunderstorms.

In situ measurements of the $\mathrm{CID}$ source region using instrumented balloons or rockets equipped with electric field meters, meteorological instrumentation, and perhaps an electric field change meter (in the case of balloon measurements) would provide valuable data in learning more about the unique discharges. The field measurements could validate or invalidate the existence of highly elevated charge densities and electric field values. Meteorological data could provide insight into potential charge separation mechanisms. Such measurements could be extremely difficult to obtain, however, since the CID source region appears to be small and sporadic in occurrence.

Further measurements of the electric field change and RF emissions from CIDs will continue to provide insight into this unique class of thunderstorm electrical 
discharges, especially if used in conjunction with weather radar data. Remote sensing methods such as these provide coverage over a large area and can offer both synoptic and small-scale perspectives. Characterization of differences between NNBPs (if they can be observed) and NPBPs would provide further insight to CID phenomenology, possibly affirming the prediction that the discharges occur at different altitudes within thunderstorms. Simply having a larger number of recorded events from which to draw conclusions would also be useful.

High accuracy locations of RF source locations during the progression of a $\mathrm{CID}$ could be extremely useful in characterizing the breakdown process and could answer the question of whether positive, negative, or bi-directional breakdown occurs. The measurements would also validate or invalidate the conclusion reached here, that the discharges are primarily vertical. These locations could be provided by a radio interferometer or even by a TOA system. The measurements would have to be made from within a few tens of kilometers of the sources in order to obtain a reasonable vertical baseline.

It would be challenging to obtain optical or acoustic data from CIDs. It is likely that the events produce light and thunder, based on their calculated physical characteristics, but the time scale of the emissions is very short and the events occur in the clouds. Optical emissions would be scattered by the clouds and rain, but might be detectable with the use of a sensitive photodiode. Acoustic emissions would be brief and possibly inaudible.

Any of the aforementioned measurements could potentially prove useful in learning more about these unique discharges. CDs are distinct thunderstorm 
electrical phenomena that will undoubtedly be the subject of future research campaigns and theoretical ponderings. 


\section{GLOSSARY OF TERMS AND ACRONYMS}

AGL: Above ground level.

ALEXIS: Acronym for Array of Low Energy X-ray Imaging Sensors. A satellite launched in April of 1993 to study soft X-rays and broadband radio signals. Also the name of the primary payload on the satellite.

BATSE: Acronym for Burst And Transient Signal Experiment. An instrument on the Compton Gamma Ray Observatory. It has served as an all-sky monitor for various types of gamma-ray and X-ray transients.

Blackbeard: A broadband radio receiver on the ALEXIS satellite. Blackbeard provided the first observations of TIPP events.

CID: Acronym for compact intracloud discharge.

CG: Cloud-to-ground.

CGRO: Acronym for Compton Gamma Ray Observatory. A satellite launched in 1991 to perform observations of celestial gamma-ray sources.

Compact intracloud discharge. A powerful source of radio signals in thunderstorms that produces NPBPs, TIPPs, and SIPPs.

CONUS: Continental United States.

CW: Continuous wave.

D-region: The portion of the ionosphere that exists between about 50 and $90 \mathrm{~km}$.

DTOA: Differential time of arrival. 
E-layer (or E-region): The portion of the ionosphere that exists between about 90 and $140 \mathrm{~km}$.

EMP: Electromagnetic pulse.

ETOA: Estimated time of arrival.

F-layer (or F-region): The portion of the ionosphere that exists above about $140 \mathrm{~km}$.

FOV: Field of view.

FWHM: Full width at half maximum.

GPS: Global Positioning System. A system of satellites that provides accurate timing and positioning information.

GOES-8: Geostationary Operational Environmental Satellite-8. A NOAA satellite at $75^{\circ} \mathrm{W}$ that provides infrared and optical imagery of the eastern US.

HF: High Frequency ( 3 to $30 \mathrm{MHz}$ ).

IC: Intracloud.

IF: Intermediate frequency.

ITCZ: Inter Tropical Convergence Zone. A region of convective activity where airmasses meet at the equator.

KSC: Kennedy Space Center.

LANL: Los Alamos National Laboratory.

LDAR: Lightning Detection and Ranging system. A system at KSC that accurately locates RF lightning pulses in three dimensions.

LF: Low Frequency (30 to $300 \mathrm{kHz}$ ).

Low-earth orbit: An earth orbit between about 300 and $2000 \mathrm{~km}$.

MDT: Mountain Daylight Time. 
MET: Mission elapsed time.

MSL: Above mean sea level.

MUF: Maximum usable frequency. The upper frequency limit that can be transmitted reliably along a given ionospherically reflected path at a given time.

Narrow positive bipolar pulse: An electric field change pulse produced by a CDD.

NEXRAD: Next Generation Weather Radar System. A nationwide system of doppler weather radars.

NIS: Nonproliferation and International Security. NIS-1 is the Space and Atmospheric Science group at LANL.

NLDN: National Lightning Detection Network. A nationwide system of receivers that provides the times and locations of lightning strikes to ground.

NMIMT: New Mexico Institute of Mining and Technology.

NNBP: Narrow negative bipolar pulse. An NPBP of opposite polarity.

NOAA: National Oceanic and Atmospheric Administration.

NPBP: Acronym for narrow positive bipolar pulse.

NUDET: Nuclear detonation.

Pegasus: A satellite launch vehicle that is dropped from an airplane at 40,000 ft.

Positive dipole model: A model of thunderstorm charge structure that features a main positive charge region above a main negative charge region. A second, smaller, positive region may underlie the negative region.

RF: Radio frequency.

RTOA: Reported time of arrival. 
Simpson Model: See positive dipole model.

SIPP: Acronym for subionospheric pulse pair.

Skip zone: A region that lies between the maximum range for line-of-sight propagation and the minimum range for 1-hop ionospheric propagation from a receiver.

Spheric: Abbreviation for atmospheric. A term usually used to describe VLF/LF radio pulses from lightning.

Subionospheric pulse pair: Paired pulse recorded by the LANL broadband HF system. Dispersed pairs were produced by CIDs. Undispersed pairs were produced by a light bulb.

TIPP: Acronym for transionospheric pulse pair.

TOA: Time of arrival.

Transionospheric pulse pair: A paired pulse recorded by the Blackbeard satellite instrument. The events are produced by CIDs and possibly by other singular, elevated, transient RF sources.

UHF: Ultra High Frequency (300 to $3000 \mathrm{MHz}$ ).

US: United States.

USGS: United States Geological Survey.

UTC: Coordinated Universal Time.

VHF: Very High Frequency (30 to $300 \mathrm{MHz}$ ).

Virtual height: The apparent reflection height of a signal reflected by the ionosphere.

VLF: Very Low Frequency ( 3 to $30 \mathrm{kHz}$ ).

VSWR: Voltage standing wave ratio. 


\section{REFERENCES}

Appleton, E. V. and M. A. F. Barnett, On some direct evidence for downward atmospheric reflection of electric rays, Proc. Roy. Soc. A, 109, 621-641, 1925.

Beasley, W. H., M. A. Uman, D. M. Jordan, and C. Ganesh, Simultaneous pulses in light and electric field from stepped leaders near ground level, J. Geophys. Res., 88, 8617-8619, 1983.

Belrose, J. S., The HF discone antenna - a frequency independent antenna for vertical polarization, QST magazine, 11-14, July 1975.

Berger, K., R. B. Anderson, and H. Kroninger, Parameters of lightning flashes, Electra, 80, 23-37, 1975.

Bils, K. R., E. M. Thomson, M. A. Uman, and D. Mackerras, Electric field pulses in close lightning cloud flashes, J. Geophys. Res., 93, 15933-15940, 1988.

Bracewell, R. N., Budden, K. G., Ratcliffe, J. A., Straker, T. W., Weekes, K., The ionospheric propagation of low- and very-low-frequency radio waves over distances less than $1000 \mathrm{~km}$, Proc. IEE, 98, Pt. III, 221-236, 1951. 
Brook, M., N. Kitagawa, and E. J. Workman, Quantitative study of strokes and continuing currents in lightning discharges to ground, J. Geophys. Res., 67, 649-659, 1962.

Brook, M. and N. Kitagawa, Radiation from lightning discharges in the frequency range 400 to $1000 \mathrm{Mc} / \mathrm{s}$, J. Geophys. Res., 69, 2431-2434, 1964.

Brook, M. and T. Ogawa, The cloud discharge, in Lightning, edited by R. H. Golde, 351-384, Academic Press Inc., New York, 1977.

Cooray, V. and S. Lundquist, On the characteristics of some radiation fields from lightning and their possible origin in positive ground flashes, J. Geophys. Res., 87, 11203-11214, 1982.

Cooray, V. and S. Lundquist, Characteristics of the radiation fields from lightning in Sri Lanka in the tropics, J. Geophys. Res., 90, 6099-6109, 1985.

Davies, K., Oblique propagation, in Ionospheric Radio, 155-207, Peter Peregrinus Ltd., London, 1990.

Dye, J. E., J. J. Jones, W. P. Winn, T. A. Cerni, B. Gardiner, D. Lamb, R. L. Pitter, J. Hallett, and C. P. R. Saunders, Early electrification and precipitation 
development in a small, isolated Montana cumulonimbus, J. Geophys. Res., 91, 1231-1247, 1986.

Eack, K. B., W. H. Beasley, W. D. Rust, T. C. Marshall, and M. Stolzenburg, Initial results from simultaneous observation of $\mathrm{X}$ rays and electric fields in a thunderstorm, J. Geophys. Res., 101, 29637-29640, 1996, a.

Eack, K. B., W. H. Beasley, W. D. Rust, T. C. Marshall, and M. Stolzenburg, X-ray pulses observed above a mesoscale convective system, Geophys. Res. Lett., 23, 2915-2918, 1996, b.

Fishman, G. J., P. N. Bhat, R. Mallozzi, J. M. Horack, T. Koshut, C. Kouveliotou, G. N. Pendleton, C. A. Meegan, R. B. Wilson, W. S. Paciesas, S. J. Goodman, and H. J. Christian, Discovery of intense gamma-ray flashes of atmospheric origin, Science, 264, 1313-1316, 1994.

Glasstone, S. and P. J. Dolan, The electromagnetic pulse and its effects, in The Effects of Nuclear Weapons, 514-540, United States Department of Defense and the Energy Research and Development Administration, Washington, D. C., 1977. 
Goodman, J. M., HF Propagation and Channel Characterization, in $\underline{\mathrm{HF}}$ Communications; Science and Technology, 171-303, Van Nostrand Reinhold, New York, 1992.

Holden, D. N., C. P. Munson, and J. C. Devenport, Satellite observations of transionospheric pulse pairs, Geophys. Res. Lett., 22, 889-892, 1995.

Holden, D. N., D. A. Smith, X. M. Shao, R. S. Massey, C. T. Rhodes, J. R. Lopez, Ground-based investigations into the sources of TIPP events, Eos, Trans., Amer. Geophys. Union, 77, F89, 1996.

Idone, V. P. and R. E. Orville, Lightning return stroke velocities in the Thunderstorm Research International Program (TRIP), J. Geophys. Res., 87, 4903-4915, 1982.

Jacobson, E. A. and E. P. Krider, Electrostatic field changes produced by Florida lightning, J. Atmos. Sci., 33, 103-117, 1976.

Kandoian, A. G., Three new antenna types and their applications, Proc. IRE, Vol. 34, 70W-75W, Feb. 1946.

Kessler, W. J. and S. P. Hersperger, Recent developments in radio location of thunderstorm centers, Bull. Amer. Meteorol. Soc., 33, 153-157, 1952. 
Kinzer, G. D., Cloud-to-ground lightning versus radar reflectivity in Oklahoma thunderstorms, J. Atmos. Sci., 31, 787-799, 1974.

Kitagawa, N. and M. Brook, A comparison of intracloud and cloud-to-ground lightning discharges, J. Geophys. Res., 65, 1189-1201, 1960.

Krehbiel, P., M. Brook, and R. McCrory, Lightning ground stroke charge location from multistation electrostatic field change measurements, Proc. of the $V$ Intern. Conf. on Atmospheric Electricity, Garmisch-Partenkirchen, Germany, 1974.

Krehbiel, P. R., M. Brook, and R. A. McCrory, An analysis of the charge structure of lightning discharges to ground, J. Geophys. Res., 84, 2432-2456, 1979.

Krehbiel, P. R., An analysis of the electric field change produced by lightning, Ph.D. dissertation, Univ. of Manchester, Instit. of Sci. and Tech., Manchester, England, 1981.

Krehbiel, P. R., R. Tennis, M. Brook, E. W. Holmes, and R. Comes, A comparative study of the initial sequence of lightning in a small Florida thunderstorm, Proc. of the VII Intern. Conf. on Atmospheric Electricity, 279-285, Am. Meteorol. Soc., Boston, Mass., 1984. 
Krider, E. P. and G. J. Radda, Radiation field wave forms produced by lightning stepped leaders, J. Geophys. Res., 80, 2653-2657, 1975.

Krider, E. P., C. D. Weidman, and R. C. Noggle, The electric fields produced by lightning stepped leaders, J. Geophys. Res., 82, 951-960, 1977.

Krider, E. P., C. D. Weidman, and D. M. Le Vine, The temporal structure of the HF and VHF radiation produced by intracloud lightning discharges, J. Geophys. Res., 84, 5760-5762, 1979.

Krider, E. P. and C. Guo, The peak electromagnetic power radiated by lightning return strokes, J. Geophys. Res., 88, 8471-8474, 1983.

Laby, T. H., J. J. McNeill, F. G. Nicholls, and A. F. B. Nickson, Waveform, energy and reflection by the ionosphere, of atmospherics, Proc. Roy. Soc. London, A174, 145-163, 1940.

Lennon, C. and L. Maier, Lightning mapping system, Proceedings of the International Aerospace and Ground Conference on Lightning and Static Electricity, NASA Conf. Publ. 3106, 89-1 to 89-10, Cocoa Beach, Fla., 1991. 
Le Vine, D. M., Sources of the strongest RF radiation from lightning, J. Geophys. Res., 85, 4091-4095, 1980.

Lin, Y. T., M. A. Uman, J. A. Tiller, R. D. Brantley, W. H. Beasley, E. P. Krider, and C. D. Weidman, Characterization of lightning return stroke electric and magnetic fields from simultaneous two-station measurements, J. Geophys. Res., 84, 6307-6314, 1979.

MacGorman, D. R., A. A. Few, and T. L. Teer, Layered lightning activity, J. Geophys. Res., 86, 9900-9910, 1981.

Maier, L., C. Lennon, P. Krehbiel, M. Stanley, and M. Robison, Comparison of lightning and radar observations from the KSC LDAR and NEXRAD radar systems, Proc. of the $27^{\text {th }}$ Conf. on Radar Meteorol., 648-650, Vail, Colo., 1995.

Maier, L., C. Lennon, P. Krehbiel, M. Maier, Lightning as observed by a fourdimensional lightning location system at Kennedy Space Center, Proc. of the $10^{\text {th }}$ International Conf. on Atmospheric Electricity, 280-283, Osaka, Japan, 1996.

Malan, D. J., Bipolar nature of the main thundercloud charges and the lower positive charge, in Physics of Lightning, 44-46, Eng. Univ. Press Ltd., London, 1963. 
Marshall, T. C. and W. D. Rust, Electric field soundings through thunderstorms, $J$. Geophys. Res., 96, 22297-22306, 1991.

Marshall, T. C., W. D. Rust, M. Stolzenburg, Electrical structure and updraft speeds in thunderstorms over the souther Great Plains, J. Geophys. Res., 100, 10011015,1995, a.

Marshall, T. C., W. Rison, W. D. Rust, M. Stolzenburg, J. C. Willett, and W. P. Winn, Rocket and balloon observations of electric field in two thunderstorms, J. Geophys. Res., 100, 20815-20828, 1995, b.

Martyn, D. F., R. O. Cherry, and A. L. Green, Long-distance observations of radio waves of medium frequencies, Proc. Phys. Soc., 47, 340-351, 1934.

Massey, R. S. and D. N. Holden, Phenomenology of transionospheric pulse pairs, Radio Sci., 30, 1645-1659, 1995.

Massey, R. S., D. N. Holden, and X. M. Shao, Phenomenology of trans-ionospheric pulse pairs, accepted for publication by Radio Sci., 1998. 
Master, M. J., M. A. Uman, W. Beasley, and M. Darveniza, Lightning induced voltages on power lines: experiment, IEEE Trans. Pow. App. Sys., 103, 25192529, 1984.

Mazur, V., Triggered lightning strikes to aircraft and natural intracloud discharges, $J$. Geophys. Res., 94, 3311-3325, 1989.

McDonald II, T. B., M. A. Uman, J. A. Tiller, and W. H. Beasley, Lightning location and lower-ionospheric height determination from two-station magnetic field measurements, J. Geophys. Res., 84, 1727-1734, 1979.

McNamera, L. F., The ionosphere, in The Ionosphere: Communications, Surveillance, and Direction Finding, 17-38, Krieger Publishing Co., Malabar, Fla., 1991.

Medelius, P. J., E. M. Thomson, and J. S. Pierce, E and DE/DT waveshapes for narrow bipolar pulses in intracloud lightning, Proc. of the 1991 International Aerospace and Ground Conference on Lightning and Static Electricity, 12-1 to 12-10, Cocoa Beach, Fla., 1991.

Oetzel, G. N. and E. T. Pierce, Radio emissions from close lightning, in Planetary Electrodynamics, edited by S. C. Coroniti and J. Hughes, 543-569, Gordon and Breach Sci. Publ., New York, 1969, a. 
Oetzel, G. N. and E. T. Pierce, VHF technique for locating lightning, Radio Sci., 4, 199-201, 1969, b.

Ogawa, T. and M. Brook, The mechanism of the intracloud lightning discharge, $J$. Geophys. Res., 69, 5141-5150, 1964.

Orville, R. E. and V. P. Idone, Lightning leader characteristics in the Thunderstorm Research International Program (TRIP), J. Geophys. Res., 87, 11177-11192, 1982.

Pierce, E. T., Atmospherics and radio noise, in Lightning, edited by R. H. Golde, 351384, Academic Press Inc., New York, 1977.

Priedhorsky, W. C., J. Bloch, D. Holden, D. Roussel-Dupré, B. Smith, R. Dingler, R. Warner, G. Huffman, R. Miller, B. Dill, and R. Fleeter, The ALEXIS small satellite project: initial flight results, Proc. SPIE, EUV, X-Ray and GammaRay Instrumentation for Astronomy IV, 2006, 114-127, 1993.

Proctor, D. E., A hyperbolic system for obtaining VHF radio pictures of lightning, $J$. Geophys. Res., 76, 1478-1489, 1971.

Proctor, D. E., Comments on "A technique for accurately locating lightning at close range", J. Appl. Meteorol., 12, 1419-1423, 1973. 
Proctor, D. E., VHF radio pictures of cloud flashes, J. Geophys. Res., 86, 4041-4071, 1981.

Proctor, D. E., Regions where lightning flashes began, J. Geophys. Res., 96, 5099$5112,1991$.

Proctor, D. E., Lightning flashes with high origins, J. Geophys. Res., 102, 1693-1706, 1997.

Ray, P. S., D. R. MacGorman, W. D. Rust, W. L. Taylor, and L. W. Rasmussen, Lightning location relative to storm structure in a supercell storm and a multicell storm, J. Geophys. Res., 92, 5713-5724, 1987.

Reynolds, S. E. and H. W. Neill, The distribution and discharge of thunderstorm charge-centers, J. Meteorol., 12, 1-12, 1955.

Rhodes, C. T., X. M. Shao, P. R. Krehbiel, R. J. Thomas, and C. O. Hayenga, Observations of lightning phenomena using radio interferometry, J. Geophys, Res., 99, 13059-13082, 1994.

Roussel-Dupré, D., J. Bloch, C. Little, R. Dingler, B. Dunne, S. Fletcher, M.

Kennison, K. Ramsey, R. King, J. Theiler, J. Sutton, and J. Wren, ALEXIS, 
the little satellite that could - 4 years later, $11^{\text {th }}$ Annual AIAA/USU Conference on Small Satellites, IV-3, Utah State University, Logan, Utah, 1997.

Roussel-Dupré, R. and A. V. Gurevich, On runaway breakdown and upward propagating discharges, J. Geophys. Res., 101, 2297-2311, 1996.

Sentman, D. D., E. M. Wescott, D. L. Osborne, D. L. Hampton, and M. J. Heavner, Preliminary results from the Sprites94 aircraft campaign: 1. Red sprites, Geophys. Res. Lett., 22, 1205-1208, 1995.

Shao, X. M., P. R. Krehbiel, R. J. Thomas, and W. Rison, Radio interferometric observations of cloud-to-ground lightning phenomena in Florida, J. Geophys. Res., 100, 2749-2783, 1995.

Shao, X. M., D. A. Smith, C. T. Rhodes, D. N. Holden, R. S. Massey, J. Lopez, M. Brook, P. R. Krehbiel, M. Stanley, R. Thomas, Observations of largeamplitude bipolar electric field change pulses: possible sources for TIPP events, Eos, Trans., Amer. Geophys. Union, 77, F87, 1996.

Shao, X. M. and P. R. Krehbiel, The spatial and temporal development of intracloud lightning, J. Geophys. Res., 101, 26641-26668, 1996. 
Simpson, G. and F. J. Scrase, The distribution of electricity in thunderclouds, Proc. Roy. Soc., A, 164, 132-150, 1938.

Smith, D. A., A multiple-channel sub-band transient detection system, M.S. Thesis, Dept. of Elec. Eng., Univ. of Colo., Boulder, Colo., 1995.

Smith, D. A. and D. N. Holden, Ground-based observations of subionospheric pulse pairs, Radio Sci., 31, 553-571, 1996.

Smith, D. A., X. M. Shao, D. N. Holden, R. S. Massey, C. T. Rhodes, and B. J. Wiemers, Observations of isolated high frequency radio bursts in association with thunderstorm activity: a possible link to TIPP events, Eos, Trans., Amer. Geophys. Union, 77, F89, 1996.

Smith, D. A., D. M. DeLapp, D. N. Holden, G. L. Stelzer, and P. L. Klingner, Calibration and evaluation of Blackbeard time tagging capability, $11^{\text {th }}$ Annual AIAA/USU Conference on Small Satellites, IV-6, Utah State University, Logan, Utah, 1997, a.

Smith, D. A., X. M. Shao, D. N. Holden, and C. T. Rhodes, Characterization of unique thunderstorm electrical discharges, Eos, Trans., Amer. Geophys. Union, 78, F77, 1997, b. 
Smith, E. K., Propagation at microwave frequencies, Science and Engineering Report No. 2, NASA Propagation Information Center, Department of Electrical and Computer Engineering, Univ. of Colo., 1991.

Stolzenburg, M. and T. C. Marshall, Testing models of thunderstorm charge distributions with Coulomb's law, J. Geophys. Res., 99, 25921-25932, 1994.

Uman, M. A., R. D. Brantley, T. T. Lin, J. A. Tiller, E. P. Krider, D. K. McLain, Correlated electric and magnetic fields from lightning return strokes, $J$. Geophys. Res., 80, 373-376, 1975.

Uman, M. A., Electric and magnetic field measurements, in Lightning, 47-113, Dover Publications, Inc., New York, 1984.

Uman, M. A., Stepped leader, in The Lightning Discharge, 82-98, Academic Press Inc., Orlando, 1987, a.

Uman, M. A., Cloud discharges, in The Lightning Discharge, 231-253, Academic Press Inc., Orlando, 1987, b.

Uman, M. A., Lightning location techniques, in The Lightning Discharge, 356-360, Academic Press Inc., Orlando, 1987, c. 
Volland, H., Longwave sferics propagation within the atmospheric waveguide, in Handbook of Atmospheric Electrodynamics, Volume II, 65-93, CRC Press, Boca Raton, 1995.

Wang, C. P., Lightning discharges in the tropics - 2. component ground strokes and cloud dart streamer discharges, J. Geophys. Res., 68, 1951-1958, 1963.

Weidman, C. D. and E. P. Krider, The fine structure of lightning return stroke wave forms, J. Geophys. Res., 83, 6239-6247, 1978.

Weidman, C. D. and E. P. Krider, The radiation field wave forms produced by intracloud lightning discharge processes, J. Geophys. Res., 84, 3159-3164, 1979.

Wescott, E. M., D. Sentman, D. Osborne, D. Hampton, and M. Heavner, Preliminary results from the Sprites94 aircraft campaign: 2. Blue jets, Geophys. Res. Lett., 22, 1209-1212, 1995.

Willett, J. C., J. C. Bailey, and E. P. Krider, A class of unusual lightning electric field waveforms with very strong high-frequency radiation, J. Geophys. Res., 94, 16255-16267, 1989. 
Willett, J. C., J. C. Bailey, C. Leteinturier, and E. P. Krider, Lightning electromagnetic radiation field spectra in the interval from 0.2 to $20 \mathrm{MHz}, J$. Geophys. Res., 95, 20367-20387, 1990.

Winn, W. P., G. W. Schwede, and C. B. Moore, Measurements of electric fields in thunderclouds, J. Geophys. Res., 79, 1761-1767, 1974.

Workman, E. J., R. E. Holzer, and G. T. Pelsor, The electrical structure of thunderstorms, NACA Tech. Note 864, 1942.

Zuelsdorf, R. S., R. J. Strangeway, C. T. Russell, C. Casler, H. J. Christian, R. C. Franz, Trans-ionospheric pulse pairs (TIPPs): their geographic distributions and seasonal variations, Geophys. Res. Lett., 24, 3165-3168, 1997. 